\title{
SHIELDING MEASUREMENTS
}

\section{FOR A 230 MEV PROTON BEAM}

\author{
by \\ Jeffrey Vincent Siebers \\ A thesis submitted in partial fulfillment \\ of the requirement for the degree of \\ Doctor of Philosophy \\ (Medical Physics) \\ at the \\ UNIVERSITY OF WISC'ONSIN-MADISON \\ 1990

\section{DISCLAIMER}

\begin{abstract}
This report was prepared as an account of work sponsored by an agency of the United States Government. Neither the United States Government nor any agency thereof, nor any of their employees, makes any wartanty, express or implied, or assumes any legal liability or responsibility for the accuracy, completeness, or usefulness of any information, apparatus, product, or process disclosed, or represents that its use would not infringe privately owned rights. Reference herein to any specific commercial product, process, or service by trade name, trademark, manufacturer, or otherwise does not necessarily constitute or imply its endorsement, recommendation, or favoring by the United States Government or any agency thereof. The views and opinions of authors expressed herein do not necessarily state or reflect those of the United States Government or any agency thereof.
\end{abstract}


(c) copyright by Jeffrey Vincent Siebers 1990

All Rights Reserved 


\section{ACKNOWLEDGEMENTS}

I acknowledge the support of the U.S. Department of Energy through contract number DE-FG02-86ER60417. I thank Loma Linda University 'Medical Center and Fermilab for financial support received for this project.

Many people have shared their talents with me, without their invaluable assistance this project would never have been completed. For their time and efforts, I express my sincere gratitude. The late Dr. Miguel Awschalom initiated this proj ct, I am grateful for his insightful ideas and the time that I was able to work with him. My thesis advisor, Professor Paul M. DeLura Jr. deserves special arognition, for he not only provided ideas, encouragement, and support, but also gave up many summer nights to assist in the data collection. Dr. David W. Pearson inerits appreciation for his assistance in equipment design and testing and for the inany nights he spent at Fermilab helping me collect data. I thank Dr. George Coutrakon for being a link between our experiment and Fermilab in the monthe prior to the experiment. George also assisted in obtaining electronic equipment, and gratefully shared scarce beam time.

Many Fermilab and Science Applications International Corporation (SAIC) employees provided invaluable assistance. Of special note, I thank Phil Young and Martin Schuize for readying beam, and Jeff Johanning and Juan Miranda for keeping it through the night. Joe McDonald of Battelle Pacific Northwest Labs lent us thisee TEPCs for these measurements and Tony Greenhouse from Lawrence Berkeley Lab lent us their TEPC'. For this, I am much obliged. I thank Dick Prael of Los Alamos National Laboratory for guidance through the LAHET 
calculations and LANL for allowing us use of their computing resources.

It has been a great pleasure to work with all the wonderful people in the department of Medical Physics at the University of Wisconsin. You have made each day enjoyable. I appreciate the valuable assistance of Nelson Chiu in preparing this document for final submission.

My family deserves special thanks for encouragement and support. Through following their models of hard work and dedication, I have completed this project. Elizabeth Zins has sustained me with love and support throughout this project. Thank you. I love you.

Gramps, this is what I know. 


\section{Contents}

Abstract

1 Introduction 1

1.1 Radiation Environment Around Proton Accelerators . . . . . . . 1

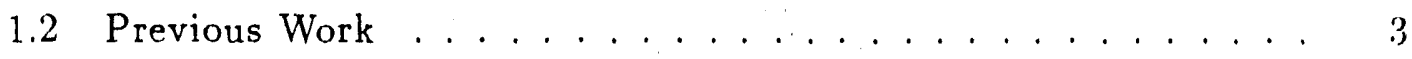

1.2 .1 Measurements . . . . . . . . . . . . . . . 3

1.2.2 Calculations..................... 6

1.3 Thesis Objectives ....................... 11

2 Materials and Methods $\quad 13$

2.1 Introduction . . . . . . . . . . . . . . . 13

2.2 Accelerator ...................... 13

2.2.1 Accelerator Components ............... 14

2.2.2 Extracted Beam Line . . . . . . . . . . . . . 14

2.2.3 Beam Energy Determination ............ 18

2.3 Shield . . . . . . . . . . . . . . . . . . . 18

2.3.1 Shielding Configuration ............. 18

2.3.2 Concrete Composition ............... 21

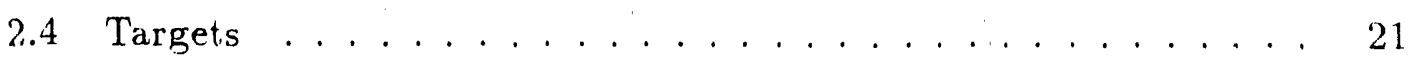


2.5 Tissue-Equivalent Proportional Counters . . . . . . . . . . . . 24

2.5.1 Principles of Operation ............. 25

2.5.2 Cavity Theory for Low Pressure TEPC . . . . . . . . . 30

2.5.3 Detector Holders ................ . . 32

2.5.4 TEPC Electronics . . . . . . . . . . . . . 34

2.5.5 Data Collection Procedures-Statistics . . . . . . . 35

2.5.6 Calibration of Low Pressure TEPC . . . . . . . . . 38

2.5.7 Error Analysis in Dose and Dose Equivalent Determination 45

2.5.8 Plotting Techniques for Proportional Counter Data . . . . 47

2.5.9 Curious Behavior of TEPCs . . . . . . . . . . 48

2.6 Beam Monitoring . . . . . . . . . . . . . . . . . . . . . 49

2.6.1 Ionization Chambers . . . . . . . . . . . . . 49

2.6.2 Ionization Chamber Calibrations ......... 55

2.6.3 Multi-Wire Proportional Counters ........... 57

2.6 .4 Polaroid Film . . . . . . . . . . . . . 58

3 Experimental Results $\quad 62$

3.1 Event Size Spectra . . . . . . . . . . . . . . . 62

3.1.1 Qualitiative Analysis of Event Spectra . . . . . . . 62

3.2 Shielding Attenuation Curves . . . . . . . . . . . . 85

4 Transport Calculation Comparisons 101

4.1 HETC-DO Calculations . . . . . . . . . . . . . . 102

4.1.1 Calculational Method ............... 102 
4.1.2 Parameterization of HETC-DO Results . . . . . . . 103

4.1 .3 Comparison with Measurements .......... 106

4.2 Analytical Methods . . . . . . . . . . . . . . . . . . . . 110

4.3 LAHET Code . . . . . . . . . . . . . . . . . . . . . . 113

4.3 .1 LAHET, A Brief Overview . . . . . . . . . 113

4.3 .2 Previous LAHET results . . . . . . . . . . . . . . . . . 114

4.3.3 Calculation Method ............... 115

4.3 .4 Geometry ... . . . . . . . . . . . . . 122

4.3 .5 Results . . . . . . . . . . . . . . . . 125

5 Conclusions

150

5.1 Measurements . . . . . . . . . . . . . . . . . . . 150

5.2 Monte Carlo Calculations . . . . . . . . . . . . . . . . 151

5.3 Summary . . . . . . . . . . . . . . . . 153

Bibliography 153 


\section{List of Tables}

2.1 Detector measuring tube locations . . . . . . . . . . . . 21

2.2 Composition of the concrete . . . . . . . . . . . 22

2.3 Target lengths and centers . . . . . . . . . . . . . . . 23

2.4 TE-propane gas mixture . . . . . . . . . . . . . . 26

2.5 TEPC gas pressures and tissue-equivalent thickness . . . . . . . 29

2.6 Materials surrounding detectors . . . . . . . . . . . . . . . 32

2.7 Run time statistics . . . . . . . . . . . . . . . . . 38

2.8 Chart of number of measurements with each target in each hole . $\quad 39$

2.9 Mean alpha particle event sizes ... . . . . . . . . . . . 41

2.10 Transmission ion chamber calibration factors . . . . . . 57

3.1 Measured dose equivalent attenuation parameters . . . . . . . . . 95

3.2 Dose equivalent, quality factors, and dose for Al target . . . . . . 98

3.3 Dose equivalent, quality factors, and dose for Fe target . . . . . . 99

3.4 Dose equivalent, quality factors, and dose for $\mathrm{Pb}$ target . . . . . 100

4.1 Concrete composition used in HETC-DO calculations . . . . . 103

4.2 Freeman's fit to HETC-DO results . . . . . . . . . . . . . . 104

4.3 Exponential fits to HETC-DO results . . . . . . . . . 105 
4.4 HETC-DO evaluated production and slope coefficients at $0^{\circ}, 22^{\circ}$, $45^{\circ}$, and $90^{\circ} \ldots \ldots \ldots \ldots \ldots$

4.5 Comparison of HETC-DO production coefficients with measure-

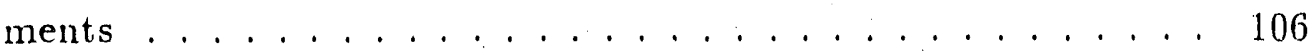

4.6 Measurements - HETC-DO mass attenuation length comparison 108

4.7 Measurements - HETC-DO attenuation length comparison . . . . 110

4.8 Attenuation parameter comparison with analytical estimation meth-

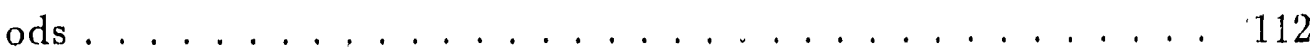

4.9 Depths for scoring events in concrete sphere for LCS calculations 127

4.10 Angular bins used in LCS calculation . . . . . . . . . . . . 128

4.11 Angular bins used for dose equivalent determination . . . . . . 128

4.12 LCS attenuation value inter-comparison . . . . . . . . . 138

4.13 LCS attenuation parameters for depths greater than $60 \mathrm{~cm}$. . . 141

4.14 Attenuation parameters determined using LCS for depths 105-210 $\mathrm{cm} \ldots \ldots \ldots \ldots \ldots \ldots$

4.15 LCS computed dose equivalent, quality factors, and close in $0^{\circ}-10^{\circ}$ interval ................. 145

4.16 LCS computed dose equivalent, quality factors, and dose in $10^{\circ}$ $30^{\circ}$ interval ....................... 146

4.17 LCS computed dose equivalent, quality factors, and dose in $30^{\circ}$ -

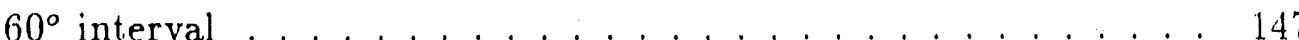

4.18 LCS computed dose equivalent, quality factors, and dose in $60^{\circ}$. $120^{\circ}$ interval ...................... 148 
4.19 LCS computed dose equivalent, quality factors, and dose in $120^{\circ}$.

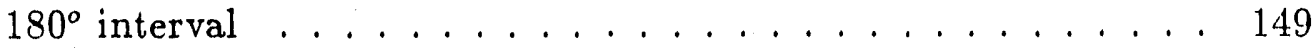

5.1 Summary of attenuation parameters . . . . . . . . . . 152 


\section{List of Figures}

1.1 Neutron yield for $113 \mathrm{MeV}$ proton incident upon $\mathrm{Fe} \ldots . . \ldots T$

1.2 Neutron yield at $7.5^{\circ}$ for $256 \mathrm{MeV}$ protons . . . . . . . . 8

1.3 HETC calculated dose attenuation curves . . . . . . . . . 9

2.1 Overhead view of experimental area . . . . . . . . 15

2.2 Side view of experimental layout . . . . . . . . . . 16

2.3 Bragg ionization curve . . . . . . . . . . . . . . . . . . . 19

2.4 Rossi type tissue-equivalent proportional counter . . . . . . . . 27

2.5 Single wire tissue-equivalent proportional counter . . . . . . . . 28

2.6 Cross-sectional view of materials surrounding detectors . . . . . . $\quad 33$

2.7 Electrical connections for one TEPC . . . . . . . . . . . . 36

2.8 Inter-connection of TEPC and beam monitoring electronics . . . 37

2.9 Layout of detectors in extraction line . . . . . . . . . . . 50

2.10 Cross-sectional views of the downstream TIC . . . . . . . . 52

2.11 Saturation characteristics of ionization chambers . . . . . . 54

2.12 Faraday cup cross-sectional view . . . . . . . . . . . 56

2.13 Schematic layout of MWPC . . . . . . . . . . . . . . 59

$2.14 \mathrm{MWPC}$ beam profiles ................. 60

$3.1{ }^{226}$ Ra gamma-ray event size spectrum . . . . . . . . . . 64 ix 
$3.214 .8 \mathrm{MeV}$ neutron event size spectrum . . . . . . . . . . 66

$3.3 \mathrm{Fe} 0^{\circ}$ event size spectra . . . . . . . . . . . . . 67

3.4 Fe $90^{\circ}$ event size spectra . . . . . . . . . . . . . . . . 69

3.5 Fe event spectra at all angles ............... 70

3.6 Fe $22^{\circ}$ event size spectra . . . . . . . . . . . . . 71

$3.7 \mathrm{Fe} 45^{\circ}$ event size spectra .................. 72

3.8 Event spectra in first penetration at $90^{\circ}$ for each target . . . . 73

3.9 Event spectra in third penetration at $90^{\circ}$ for each target. . . . . 74

$3.10 \mathrm{~Pb} 0^{\circ}$ event size spectra . . . . . . . . . . . 76

$3.11 \mathrm{~Pb} 22^{\circ}$ event size spectra. . . . . . . . . . . . 77

$3.12 \mathrm{~Pb} 45^{\circ}$ event size spectra. . . . . . . . . . . . 78

$3.13 \mathrm{~Pb} 90^{\circ}$ event size spectra. . . . . . . . . . . . . 79

$3.14 \mathrm{Al} 0^{\circ}$ event size spectra . . . . . . . . . . . . . . 80

3.15 A1 $22^{\circ}$ event size spectra . . . . . . . . . . . . 81

$3.16 \mathrm{Al} 45^{\circ}$ event size spectra . . . . . . . . . . . . . . 82

$3.17 \mathrm{Al} 90^{\circ}$ event size spectra . . . . . . . . . . . . . . 83

3.18 Residual soft spectrum at $90^{\circ}$ after subtracting hard components 84

3.19 In air event size spectra $0^{\circ} \ldots \ldots \ldots$. . . . . . . . 86

3.20 In air event size spectra $22^{\circ} \ldots \ldots \ldots$. . . . . . . . . 87

3.21 In air event size spectra $45^{\circ} \ldots \ldots \ldots$. . . . . . . . . 88

3.22 In air event size spectra $90^{\circ} \quad \ldots \ldots . \ldots 96$

3.23 In air event size spectra $142^{\circ} \ldots \ldots$. . . . . . . . . . . 90

$3.24 \mathrm{Al}$ target dose equivalent attenuation in concrete . . . . . . . 91 
$3.25 \mathrm{Fe}$ target dose equivalent attenuation in concrete . . . . . . . . 92

$3.26 \mathrm{~Pb}$ target dose equivalent attenuation in concrete . . . . . . 93

3.27 Dose equivalent values at $0^{\circ}$ for each target $\ldots \ldots 96$

4.1 HETO-DO prediction of dose equivalent values at equivalent shield mass thicknesses . . . . . . . . . . . . . . . . . 109

4.2 HETC-DO prediction of dose equivalent values versus shield depth 111

4.3 Neutron spectrum produced by $113 \mathrm{MeV}$ protons incident upon an Fe target ......................... 116

4.4 Ratio of computed and measured neutron yields for $113 \mathrm{MeV}$ protons on $\mathrm{Fe} \ldots \ldots \ldots \ldots \ldots$. . . . . . . . . . . . . . .

4.5 Calculated and measured neutron yield for $256 \mathrm{MeV}$ protons incident upon an Fe target. . . . . . . . . . . . . . 118

4.6 Ratio of computed and measured neutron yields for $256 \mathrm{MeV}$ Protons on $\mathrm{Fe} \ldots \ldots \ldots \ldots \ldots$

4.7 Neutron spectra puoduced by $256 \mathrm{MeV}$ protons incident upon $\mathrm{Fe}$ and LAHET calculated yield without inciusion of the preequilibrium model . . . . . . . . . . . . . . . . . 120

4.8 Neutron fluence-to-dose equivalent conversion factors . . . . . . . 123

4.9 Neutron fluence-to-absorbed dose conversion factors . . . . . . 124

4.10 Geometry used in LCS calculation . . . . . . . . . . . 126

4.11 LCS computed target high energy emission neutron spectrum . . 130

4.12 LCS computed target emission low energy nentron spectrum . . 131 
4.13 Photon spectra crossing into the concrete for $230 \mathrm{MeV}$ protons incident upon a Fe target calculated using LCS. . . . . . . . . . 132

4.14 LCS calculated neutron spectra $105 \mathrm{~cm}$ in the concrete for 230 $\mathrm{MeV}$ protons incident upon an $\mathrm{Fe}$ target . . . . . . . . . . . 133

4.15 LCS calculated low energy neutron spectra $105 \mathrm{~cm}$ in the concrete for $230 \mathrm{MeV}$ protons incident upon an Fe target . . . . . . . 134

4.16 Photon spectra $105 \mathrm{~cm}$ in the concrete for $230 \mathrm{MeV}$ protons incident upon a Fe target calculated using LCS . . . . . . . . . . 1355

4.17 LCS computed dose equivalent values versus shield depth . . . . 137

4.18 LCS computed dose equivalent values and fitted curves . . . . . . 139

4.19 Ratio of calculation to measurements versus shield thickness . . . 140

4.20 Angular dependence of neutron production . . . . . . . . . . 144 


\title{
Abstract \\ SHIELDING MEASUREMENTS \\ FOR A 230 MEV PROTON BEAM
}

\author{
Jeffrey Vincent Siebers \\ Under the supervision of Professor Paul M. DeLuca Jr.
}

Energetic secondary neutrons produced as protons interact with accelerator components and patients dominate the radiation shielding environment for proton radiotherapy facilities. Due to the scarcity of data describing neutron production, attenuation, absorbed dose, and dose equivalent values, these parameters were measured for $230 \mathrm{MeV}$ proton bombardment of stopping length $\mathrm{Al}, \mathrm{Fe}$, and $\mathrm{Pb}$ targets at emission angles of $0^{\circ}, 22^{\circ}, 45^{\circ}$, and $90^{\circ}$ in a thick concrete shield. Low pressure tissue-equivalent proportional counters with volumes ranging from $1 \mathrm{~cm}^{3}$ to $1000 \mathrm{~cm}^{3}$ were used to obtain microdosimetric spectra from which absorbed dose and radiation quality are deduced. Dose equivalent values and attenuation lengths determined at depth in the shield were found to vary sharply with angle, but were found to be independent of target material.

Neutron dose and radiation length values are compared witis Monte Carlo neutron transport calculations performed using the Los Alamos High Energy Transport Code (LAHET). Calculations used $230 \mathrm{MeV}$ protons inciclent upon an Fe target in a shielding geometry similar to that used in the experiment. LAHET calculations overestimated measured attenuation values at $0^{\circ}, 22^{\circ}$, and 
xiv

$45^{\circ}$, yet correctly predicted the attenuation length at $90^{\circ}$. Cor arson of the mean radiation quality estimated with the Monte Carlo calculations with easurements suggest that neutron quality factors should be increased by a factor of 1.4. These results are useful for the shielding design of new facilities as well as for testing neutron production and transport calculations.

$$
\text { Fard hillihecos. }
$$




\section{Chapter 1}

\section{Introduction}

Medical imaging modalities have advanced to the point that the precise location of patient tumors is obtainable. The time has come for use of heavy charged particles in radiation therapy, providing the ability to precisely deposit radiation dose due to the Bragg peak phenomenum. Control systems now allow heavy charged particle accelerators to operate unattended. One of the remaining problems to be dealt with in heavy charged particle radiotherapy is the protection of personnel from the radiation field produced as the primary heavy charged particle beam interacts with matter. This thesis addresses this problem through determination of the attenuation characteristics of the secondary radiation field in concrete.

\subsection{Radiation Environment Around Proton Accelerators}

Radiation hazards associated with $70-250 \mathrm{MeV}$ protons used in radiotherapy are not due to the primary protons. Rather, they are due to secondary neutrons produced as protons interact with accelerator components and the patient. Protons interact with matter in two general ways. 
(1) Coulomb collisions with atomic electrons. Even though collisions with atomic electrons transfer an average of only a few electron-volts per interaction, due to the large number of interactions, this results in the greatest energy loss per unit path-length for the protons. Energetic electrons, $\delta$-rays, produced in the proton-electron interactions are of short range and deposit their energy locally.

(2) Nuclear interactions within the target material. While not the primary cause of proton energy degradation, nuclear interactions produce the radiation hazard for proton accelerators. Proton induced nuclear interactions with the target consist of elastic and non-elastic interactions.

In elastic interactions, incident protons interact with the coulomb force of the nucleus. No kinetic energy is lost in the center of mass. For interactions with heavy nuclei, the incident proton typically loses very little energy in the lab frame and is scattered at a small angle.

For non-elastic interactions, protons enter the nucleus and interact directly with a single nucleon or a group of nucleons. Interactions with a group of nucleons results in the formation of an excited compound nucleus. The likely decay mechanism of a compound nucleus is nucleon evaporation followed by $\gamma$-ray emission. Evaporative nucleons are emitted isotopically and are typically of fairly low energy $(<15 \mathrm{MeV})$. Direct interactions between an incident proton and a single nucleon may result in large energy transfers. The struck nucleon, as well as the incident proton, then proceed through the nucleus, possibly striking other nụcleons, resulting in an intranuclear cascade. Following an intranuclear cascade, the residual nucleus is likely to be in an excited state which can decay via 
eviporation processes. Between these two processes, preequilibrium emission can occur in which a nucleon is emitted after some collisions, but before the energy is distributed amongst the nucleons in the compound nucleus.

Cascade neutrons are of nigher energy than the preequilibrium and evaporation neutrons with energies up to the incident proton energy. Due to conservation of momentum, these nucleons are predominantly emitted at small angles in the forward hemisphere. Preequilibrium neutrons are also forward directed, but less so.

In summary, the radiation environment produced by primary protons consists mainly of secondary protons and neutrons. Absorption of the secondary protions proceeds similarly to that of the primary proton beam. Due to the large mean free paths of high energy neutrons, neutron atitenuation requires thick shields.

\subsection{Previous Work}

\subsubsection{Measurements}

For protons with 70-250 MeV kinetic energy, there are very little data and few calculations for the neutron attenuation in thick shields. The only shidding measurement made in a good geometry in this energy region was by Distenfeld [44] who determined the neutron attenuation in water at $90^{\circ}$ for a $200 \mathrm{MeV}$ incident proton beam at Brookhaven National Laboratory. Measurements used activation detectors. The attenuation of each energy group was observed and the approximate equilibrium neutron spectrum in the shield deduced. ICRI 20 Huence to dose conversion factors were applied to determine the dose equivalent 
in the water [60].

Also of interest are neutron spectra and yield measurements. Wachter et al. [10T] measured neutron spectra from $160 \mathrm{MeV}$ proton bombardment of various targets, and compared their results with Monte Carlo intranuclear cascade calculations made by Bertini [25]. General agreement between experimental and theoretical results was observed. However, Wachter found that calculations overestimate neutron yields for thick targets, low energies and small angles. Bertini $[28,29]$ reviewed many measurements and discovered anomalies between various measured neutron spectra as well as disagreement with his Monte Carlo calculated spectra for $95-250 \mathrm{MeV}$ protons. For $\mathrm{E}_{p}>300 \mathrm{MeV}$, he found general agreement between calculated and measured spectra.

Neutron spectral measurements were done by Broome et al. for $72 \mathrm{MeV}$ protons incident upon a copper beam stop [35]. Broome reported neutron yields, unfolded the neutron spectrum from activation detector data, and found that the Monte Carlo code High Energy Transport Code (HETC) $[15,68]$ overestimates neutron production for high energies and small angles. At high energies, HETC uses Bertini's intranuclear cascade code [24] and Guthrie's evaporation program [52] to determine the energy and direction of emitted particles. Low energy neutrons ( $<15 \mathrm{MeV})$ are transported using a modified version of the Monte Carlo code O5R [65].

The evaporation component of the neutron spectrum produced by low energy protons was measured and calculated by Alsmiller et al. $|6|$. Both the intranuclear cascade code of Bertini and the Evap-4 code of Guthrie were found to 
underestimate the evaporation spectrum.

Thick target neutron spectra obtained by Fasso et al. [48] for $50 \mathrm{MeV}$ protons incident upon a variety of targets suggest. Alsmiller's [1] fits to Bertini's intranuclear cascade data [24] overestimat: the neuiron production at small angles and underestimate the neutron production at large angles.

Nakamura [79] compared neutron spectra produced by 30 and $52 \mathrm{MeV}$ protons bombarding various targets with spectra obtained directly from Bertini's intranuclear cascade calculation. The calculated spectral shapes at a given angle were observed to be similar to the measurements. However, calculated values overestimate cascade components in the forward direction by factors of 2-3, yet underestimate the evaporation components at large angles, confirming Fasso's findings. Bertini's intranuclear cascade calculation is based upon the Fermi free gas model of the nucleus. Nakamura noted that due to nuclear structure effects below $\approx 100 \mathrm{MeV}$, the Fermi free gas model is inadequate. Therefore, neutron spectra calculated by this technique are incorrect.

For higher energy protons, $E_{p}=500 \mathrm{MeV}$, incident upon an Fe target, Ban [22] found that HETC overestimates the cascade neutron production in the forward direction.

Recently, investigators at Los Alamos completed neutron yield measurements for 113 and $256 \mathrm{MeV}$ protons incident upon a variety of targets $[73,74,75]$. Comparison with HETC calculations showed that at $113 \mathrm{MeV}$, HETC overestimates the high energy neutron production at small angles and underestimates neutron production at large angles (Figure 1.1). At $256 \mathrm{MeV}$, the high energy neutron 
production was estimated correctly by $\mathrm{HETC}$, but the large angle neutron production was still grossly underestimated. Figure 1.2 shows a plot of the neutron yield observed at $7.5^{\circ}$ for $256 \mathrm{MeV}$ protons bombarding a variety of targets.

\subsubsection{Calculations}

Alsmiller et al. [8] calculated the neutron shielding necessary for $200 \mathrm{MeV}$ protons incident upon an aluminum target. Neutron production spectra were obtained using the analytical representation of the neutron production obtained from Bertini's cascade calculation [1] and integrating the neutron pioduction over the proton slowing down spectrum in several angular bins. ANISN [46], a one dimensional discrete ordinates code, was used to calculate the attenuation in the shield. The neutron spectrum obtained for each angular bin was used as an isotropic source in the center of a spherical concrete shield for an ANISN run. Dose equivalent was determined as a function of radius in the shield. Results from the computed neutron dose equivalent attenuation in ordinary concrete are

shown in Figure 1.3. Alsmiller also analysed Distenfeld's [44] measurements and found good agreement with his calculations.

While Alsmiller et al. performed several other calculations for neutron shielding and shielding for proton accelerators, $|1|-[i]$, the 1975 work summarized the applicable results from these earlier calculations.

Other than Alsmiller's efforts, little work has been done on shielding calculations for proton accelerators. Braid [33] developed an approximation procedure based upon one of Alsmiller's earlier calculations [1]. Smith [98] commissioned 


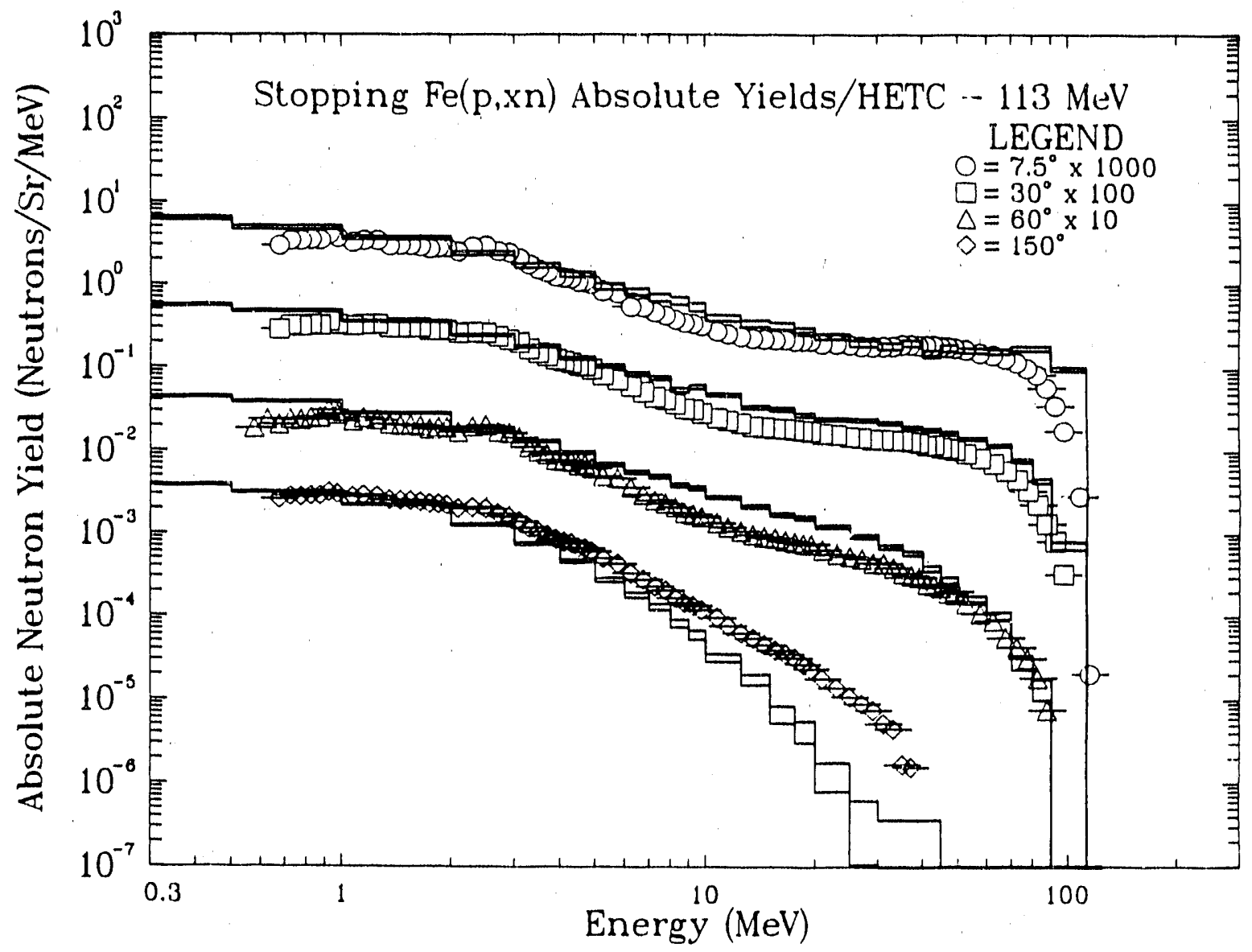

Figure 1.1: Neutron yield at $7.5^{\circ}, 30^{\circ}, 60^{\circ}, 120^{\circ}$, and $150^{\circ}$ produced by $113 \mathrm{MeV}$ protons bombardment of a thick Fe target. Results of the HETC calculation are shown as histograms. Data and calculations courtesy of Meier [74]. 


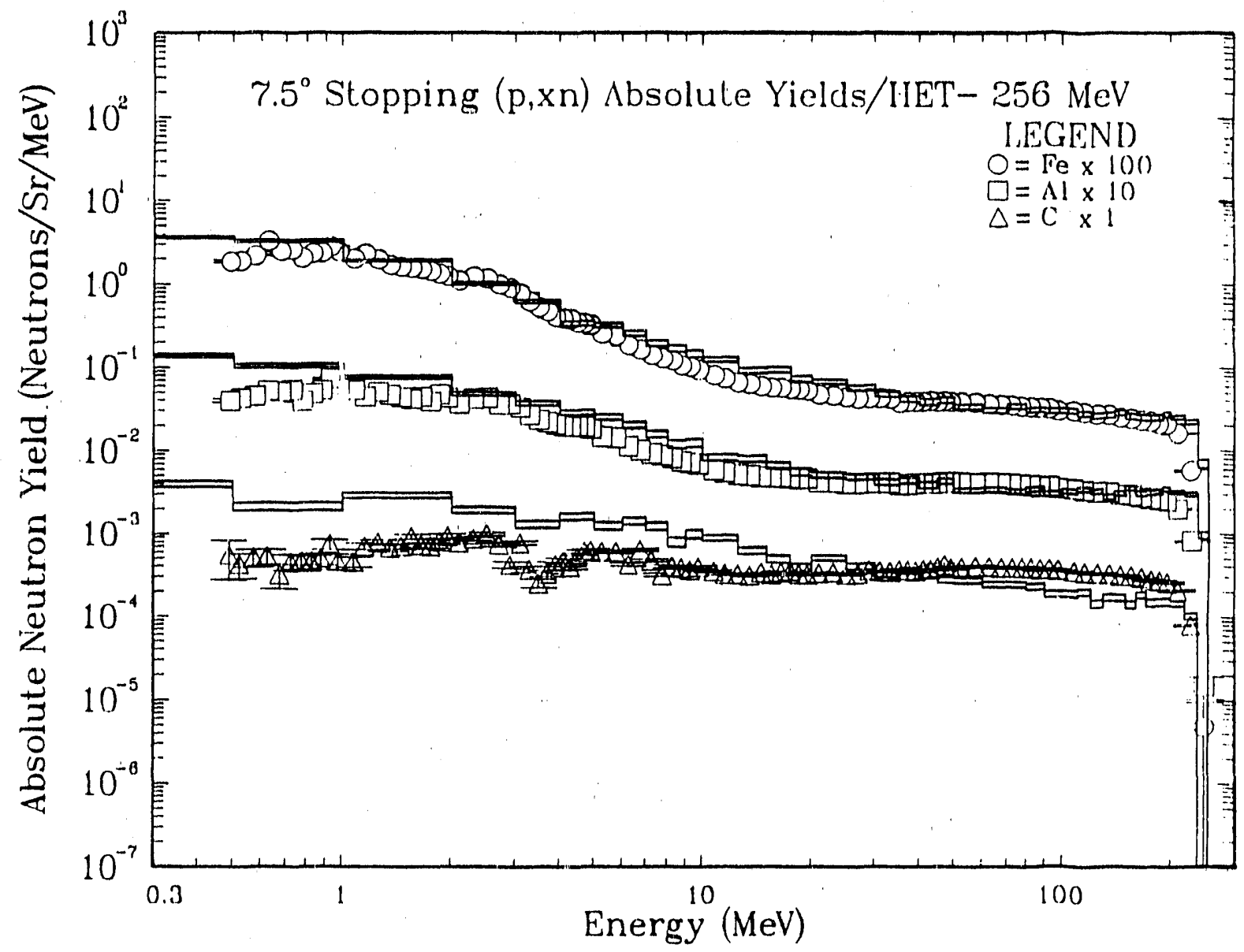

Figure 1.2: Measured neutron yield at $7.5^{\circ}$ produced by $256 \mathrm{MeV}$ protons bombardment of a variety of stopping-length targets. Results of the HETC calculation are shown as histograms. Graph reproduced from Meier [75]. 


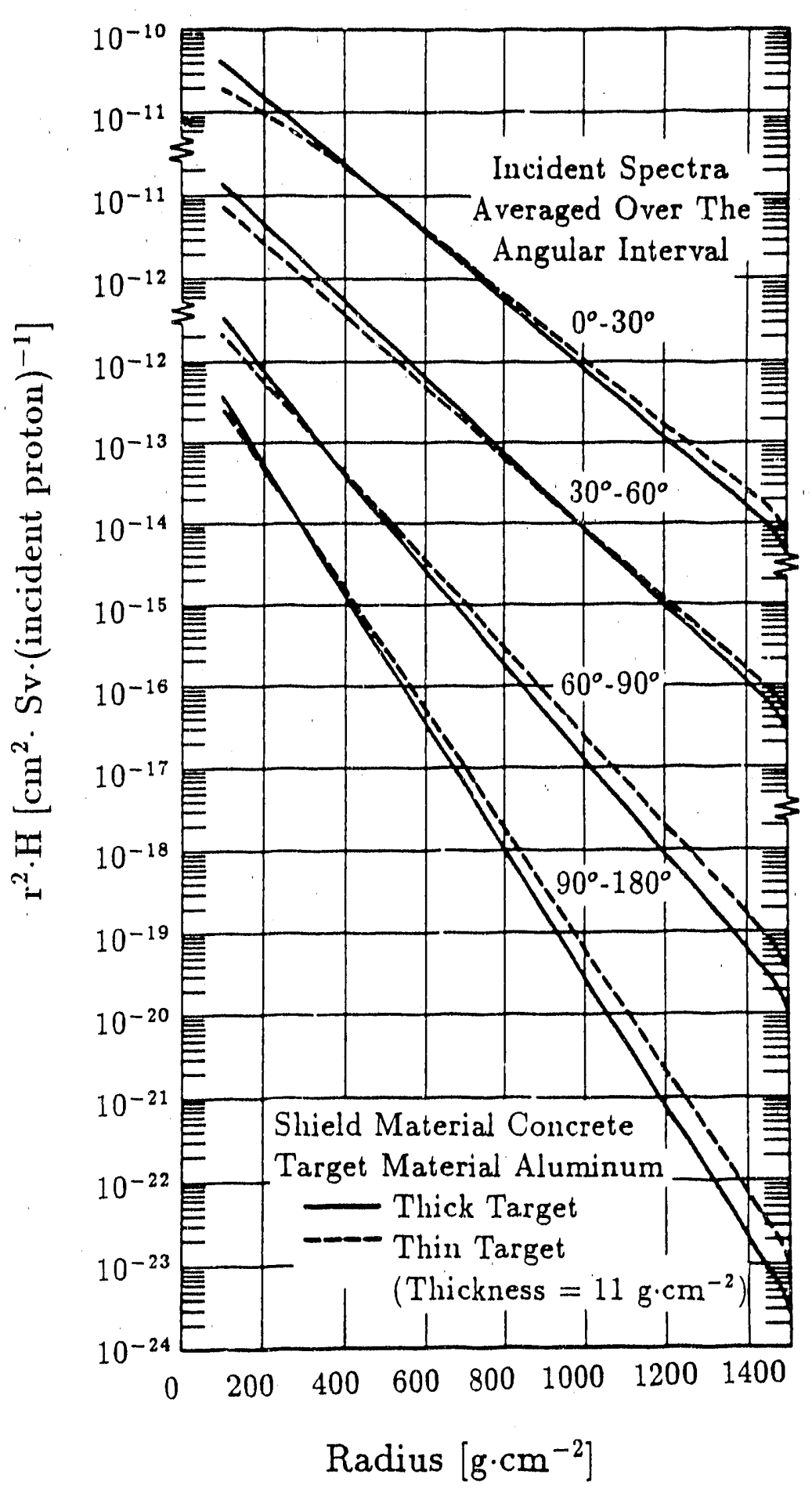

Figure 1.3: HETC' calculated dose equivalent multiplied by $r^{2}$ "s radius in a spherical concrete shield for $200 \mathrm{MeV}$ protons incident upon an Al target. Drawing reproduced from ORNL-DWG $74-12286$ [8]. 
Alsmiller to provide shielding data for the Indiana University $200 \mathrm{MeV}$ proton cyclotron facility. Smith used spectra and transport calculations provided by Alsmiller to determine the dose-equivalent at depth using ICRU flux to dose conversion coefficients [60]. Smith also interpreted Alsmiller's results in a manner useful for angular interpolations through definition of an effective angle for each angular interval.

Tesch $[105]$ compiled available shielding measurements and calculations to obtain estimations for the shielding required at $90^{\circ}$ for $50-1000 \mathrm{MeV}$ proton accelerators. Recently, Awschalom [21] extrapolated Alsmiller's data to different target materials and higher beam energies.

Most recently, Hagen et al. (54) repeated Alsmiller's calculations for the shielding design of a $70-250 \mathrm{MeV}$ proton syuchrotron. Neutron attenuation calculations in concrete for 150,200 and $250 \mathrm{MeV}$ protons incident upon iron and water were done in smaller angular bins than in Alsmiller [8]. HETC was used to compute the neutron production in the target. These calculations essentially reproduced Alsmiller's data set with more recent interaction cross sections and smaller angular intervals. Their results confirmed the extrapolation estimates of Awschalom [21]. The proton radiotherapy facility at Loma Linda University Medical Center was constructed using the results of these calculations.

The input neutron spectra for all shielding calculations were rerived either directly or indirectly from Bertini's intranuclear cascade calculation. As has been shown in Section 1.2.1, this calculation provides an incorrect incident neutron spectrum. Since the estimated spectrum is harder in the forward direction, 
thicker shields than necessary would result from computations in this region. At large angles, neutron yields are underestimated, therefore calculations would underestimate required shielding thickness.

\subsection{Thesis Objectives}

The objective of this work was to provide a basis data set upon which the radiation shielding required for proton radiotherapy accelerators can be designed. To this end, the energy deposited per unit mass, more commonly referred to as absorbed dose, was measured and is reported as a function of depth and angle for the radiation environment produced when $230 \mathrm{MeV}$ protons strike stopping length $\mathrm{Al}, \mathrm{Fe}$, and $\mathrm{Pb}$ targets. Radiobiologically, the most interesting aspect is the effect produced by the energy deposition. Hence the measurements included examination of the spectrum of energy deposition pe: unit path-length and used this quantity to determine radiation quality. The product of the absorbed close and radiation quality, referred to as absorbed dose equivalent, is also reported at each penetration. The dose equivalent attenuation in the shield is then deduced.

A second goal of this project was to determine the ability of calculations based upon nuclear physics models to predict the experimental results. Therefore, results were compared with calculations based upon High Energy Transport. Code coupled with a discrete ordinates transport calculation completed for the shielding design of the Loma Linda Mediral Center proton therapy facility. Difficulties and differences found in the comparison between the experimental and calculational results are discussed. Further Monte Carlo calculations based upon 
a more complete nuclear model were performed as part of this thesis and are also discussed. In this calculation, absorbed dose, dose equivalent, and neutron spectra are estimated and used to derive the mean radiation quality. The dose equivalent attenuation parameters computed are compared with those found using the measurements.

The overall objective of this work was to provide reliable data to health physicists responsible for designing shielding for proton radiotherapy accelerators. Only time and continued measurements can provide a measure of the success of this goal. 


\section{Chapter 2}

\section{Materials and Methods}

\subsection{Introduction}

To provide shielding data for proton radiotherapy accelerators, measurements of the dose attenuation in a concrete shield were made for $230 \mathrm{MeV}$ protons stopping in $\mathrm{Al}, \mathrm{Fe}$, and $\mathrm{Pb}$ targets. Dose and dose equivalent were measured as a function of depth in the concrete shield at angles of $n^{\circ}, 22^{\circ}, 45^{\circ}$ and $90^{\circ}$. This section describes the accelerator, experimental geometry, detectors, as well as the experimental methods used in data acquisition.

\subsection{Accelerator}

In these experiments, the accelerator used was a variable energy [70-250 MeV] proton synchrotron developed for Loma Linda University by Fermi National Accelerator Lab. Measurements were made during accelerator commissioning at Fermilab. After commissioning completion, the accelerator was disassembled and shipped to Loma Linda, where it is being reassembled for use in their proton radiotherapy facility. Figures 2.1 and 2.2 shows schematic layouts of the accelerator and extracted beam line as setup at Fermilab. The synchrotion had 
a cycle time of eight seconds at Fermilab and will have a two second cycle time when in use at Loma Linda.

Accelerator operation is described in detail in the Loma Linda University Proton Therapy Facility Operations Manual [38].

\subsubsection{Accelerator Components}

The injection line consists of a duoplasmatron ion-source after which the $30 \mathrm{keV}$ beam is focused into the entrance of a $425 \mathrm{MHz}$ radiofrequency quadrupole linear accelerator (RFQ) which accelerates the beam to $2 \mathrm{MeV}$. Downstream of the $\mathrm{RFQ}$, the beam travels through several quadrupole focussing magnets to a $180^{\circ}$ dipole which bends the beam towards the accelerator. The beam is then bent vertically down $25^{\circ}$ then back up $20^{\circ}$ by a septum magnet and $5^{\circ}$ by a kicker to bring the beam to level in the accelerator main ring. The main acceleration ring consists of eight dipole magnets (two per corner), a radiofrequency ravity for acceleration, quadrupoles for focusing and extraction, a few dipole steering magnets, and an electrostatic septum and Lambertson magnet for beam extraction.

The ring dipole magnet excitation ramp begins when injection into the ring occurs. The beam is accelerated to $233 \mathrm{MeV}$ in one second and then stored for about one second at which time extraction began.

\subsubsection{Extracted Beam Line}

Protons are extracted from the accelerator in a two step process. First, an electrostatic septum deflects a portion of the beam outwards. Then, after the 


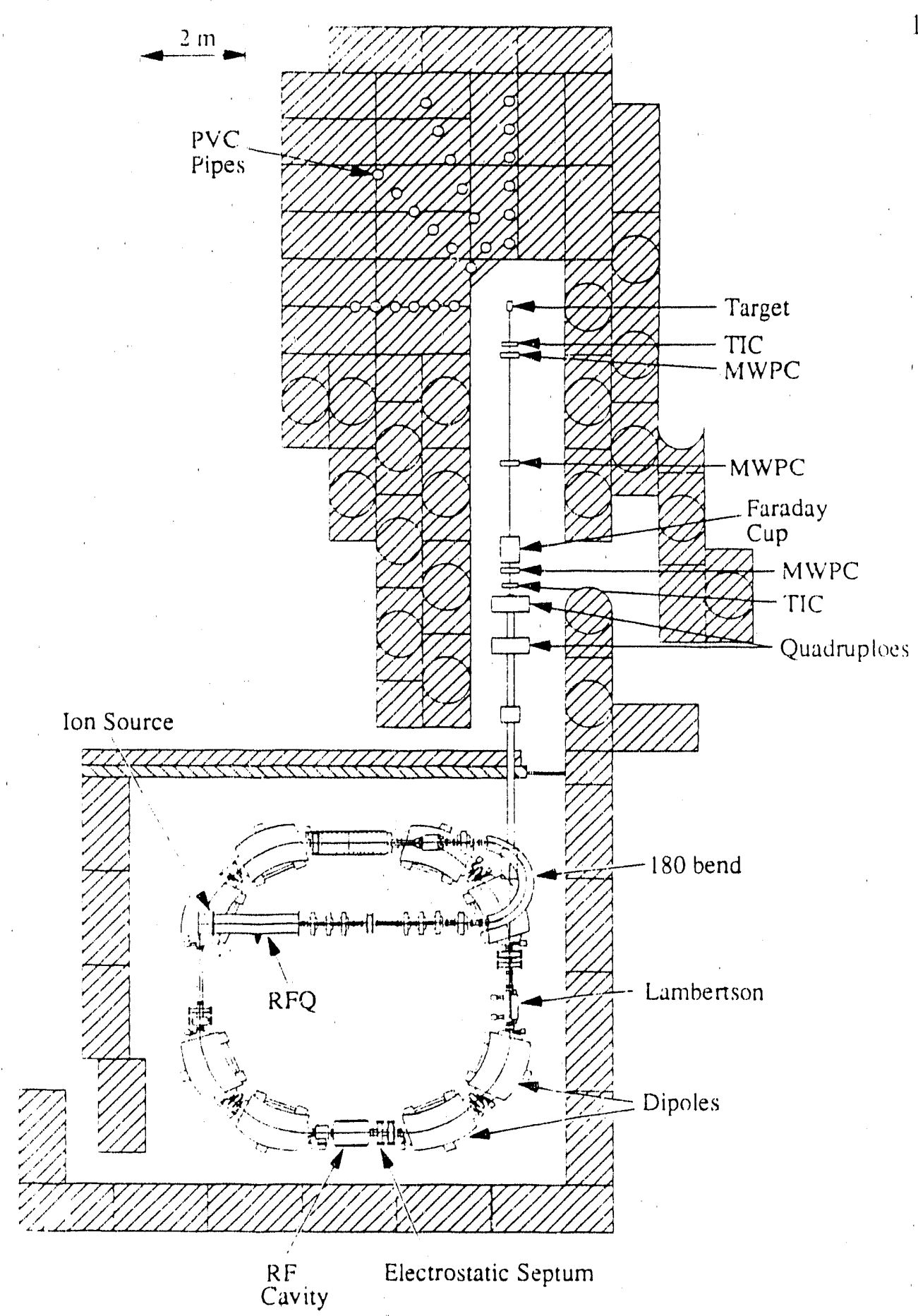

Figure 2.1: Overhead schematic view of the Loma Linda $70-350$. Me V proton synchrotron and extracted beam line as set up at Fermilab for these exprerinents. In

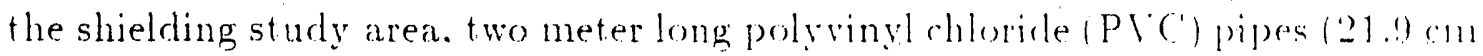

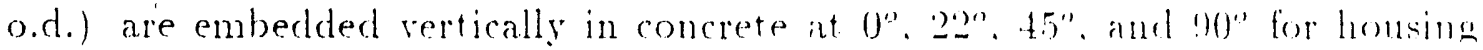
detectors during measurements. The first pipe is cast $=3.32 \mathrm{~cm}$ in the dipection of neutron penetration. Distances between pipe centers are listed in Table 2.1. 


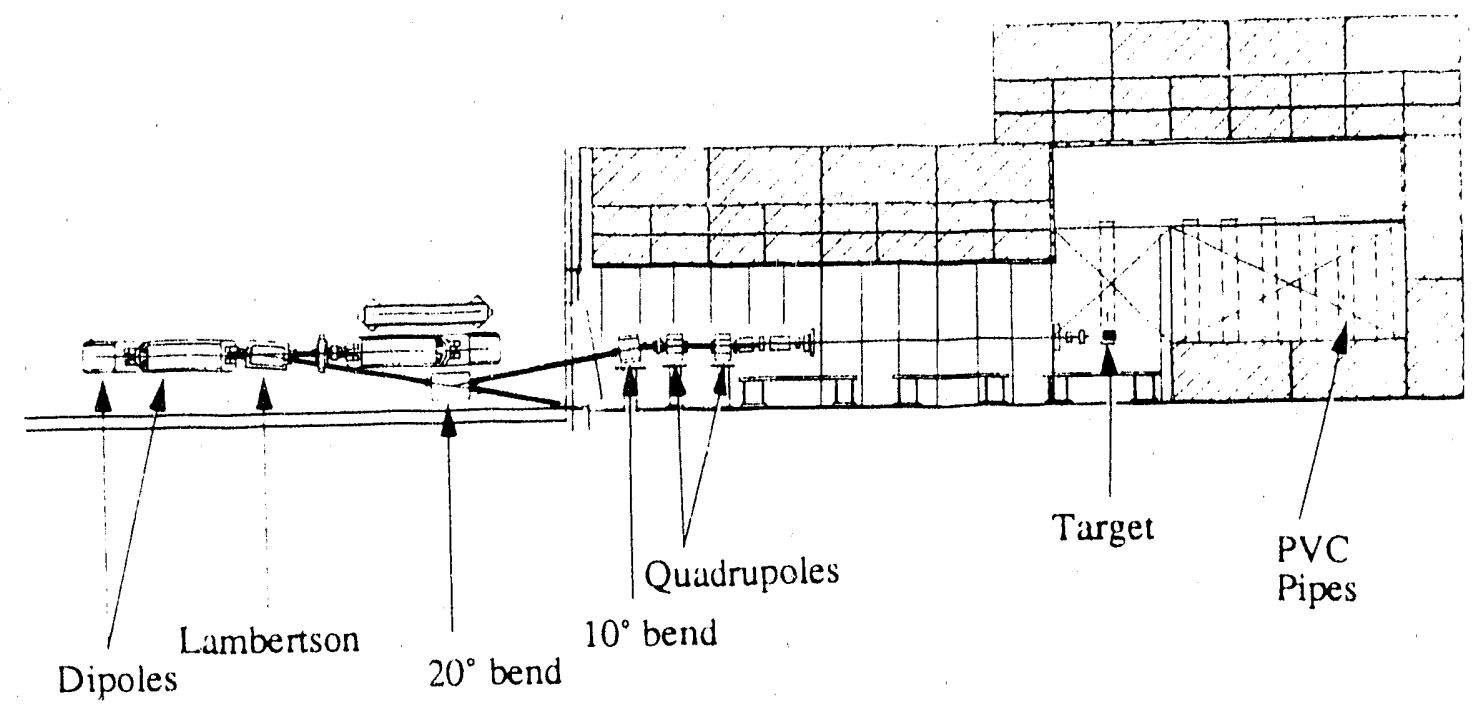

Figure 2.2: Side schematic view of the Loma Linda $70-250$ MeV proton synchrotron and extracted beam line as set up at Fermilab. In the area used for these shielding measurements, the shield height is $2.9 \mathrm{~m}$. I wo meter fong PV(! sewer pipes (21.9 cm (1.d.) are embediled vertically in comcrete to allow detectors to be lowered into the shield. 
beam corners the dipole, the extraction Lambertson magnet bends the beam $10^{\circ}$ downward, out of the accelerator. The beam travels to a $20^{\circ}$ bend, which, with the help of vertical and horizontal steering magnets, directs the beam out into the extracted-beam area. In this area, a $10^{\circ}$ magnet bends the beam back to level (Figure 2.2). Next the beam experiences a quadrupole doublet which focuses the beam to the target area. The beam exits the accelerator vacuum after the final quadrupole and travels through air to the target location.

Since the electrostatic septum deflects only a small portion of the stored beam, many $\left(\approx 7.5 \cdot 10^{6}\right)$ turns are required to extract the entire beam. Extraction lasts about $0.8 \mathrm{~s}$. The next $5.2 \mathrm{~s}$ are spent returning supplies to injection levels for the next beam pulse. During the neutron shielding measurements, extracted current increased from about $10^{8}$ protons $p$ pulse ${ }^{-1}$ to $10^{10}$ protons pulse ${ }^{-1}$. Extraction efficiency also rose from about $30 \%$ to about $90 \%$ of the protons available in the ring.

After leaving the accelerator vacuum at the quadrupole exit, the beam travels down the extracted-beam line through various monitoring devices. An upstream transmission ion chamber (TIC) monitors the beam intensity and a multi-wire proportional counter monitors the beam position. The beam then passes through various detectors needed for other studies and continues to a downstream TIC and another multi-wire proportional counter for measuring the beam intensity and position. These detectors are described in detail in Section 2.6. After passing through the downstream TIC, the beam strikes the neutron producing larget. 


\subsubsection{Beam Energy Determination}

The proton beam energy incident upon the target was determined through measurement of the proton range in water. A $0.3 \mathrm{~cm}^{3}$ parallel plate ionization chamber ${ }^{1}$ is aligned on the beam axis and scanned along the beam direction in a water tank. The ion chamber output is plotted versus the water thickness traversed (Figure 2.3) to get the resulting Bragg ionization curve. Beam energy is determined through comparison of the measured beam range with interpolations of range-energy calculations [67]. Incident proton energy was determined to be $230 \mathrm{MeV}$. Beam energy in the accelerator was determined by consideration of the materials traversed before the beam entered the water tank and adding their thicknesses to the measured beam range. Accelerator proton energy was found to be $233 \mathrm{MeV}$.

Proton energy can also be estimated using the frequency of revolution and the ring circumference. Using these parameters, proton energy in the ring is calculated to be $232.8 \mathrm{MeV}$, in excellent agreement with our measured value.

\subsection{Shield}

\subsubsection{Shielding Configuration}

The radiation shielding measurements were made in air and at various depth within a concrete shield at the end of an extracted beam cave constructed outside of the accelerator area at Fermilab. The majority of the extraction cave was

\footnotetext{
${ }^{1}$ Exradin Model P11, Manufactured by Exradin, Lisle, Illinois.
} 


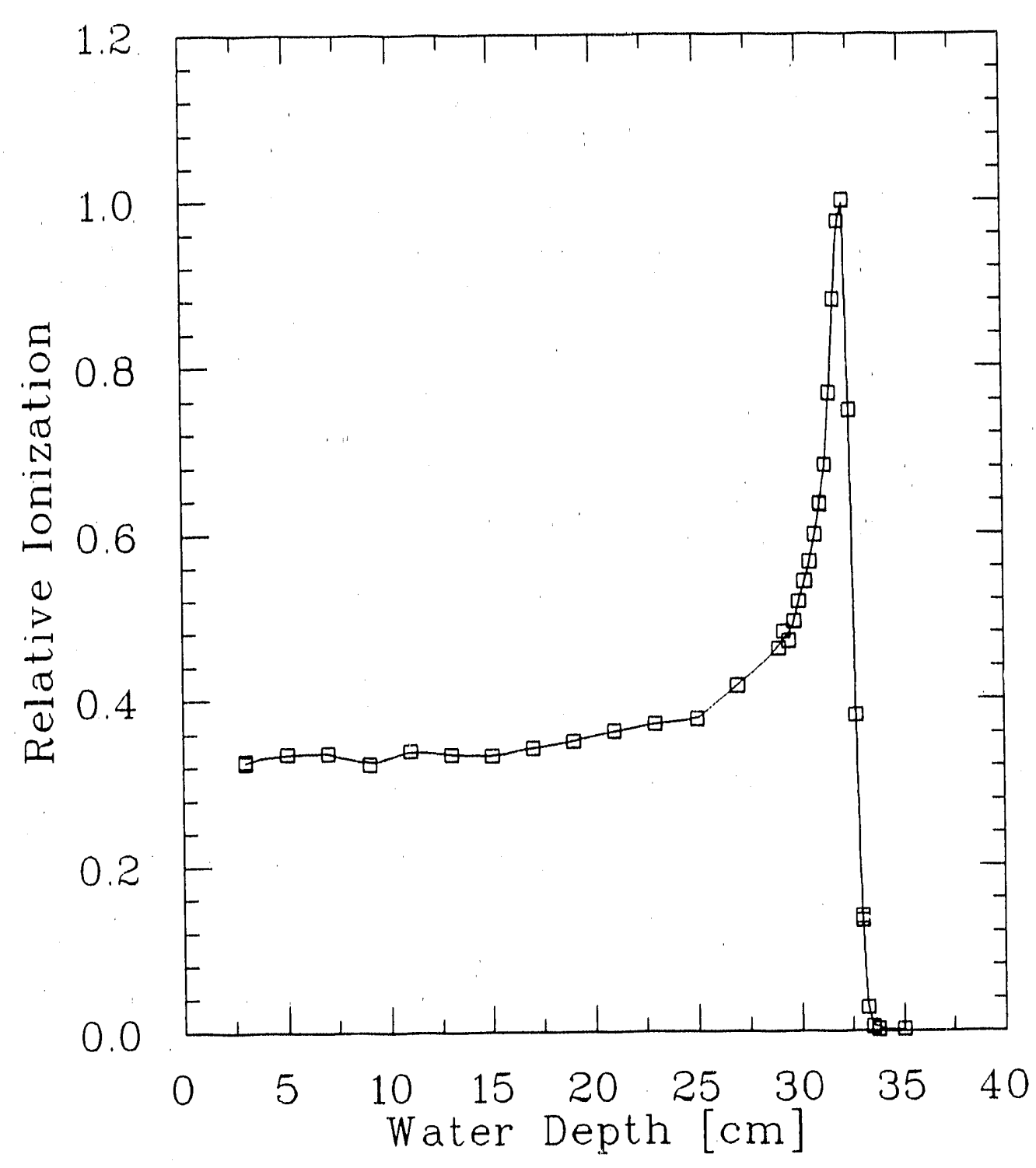

Figure 2.3: Bragg ionization curve measured using a scanning ionization chamber in a water tank from which beam energy is determined. Depths shown are water thickness traversed. 
shielded using conventional concrete shielding blocks available at Fermilab. The area for these shielding measurements was poured in place. Concrete is used for shielding since it is relatively inexpensive and is typically used in building construction. It also includes a large hydrogen content for slowing down and absorbing neutrons, and contains moderately high atomic number components for the absorption of capture gamma rays.

Figures 2.1 and 2.2 show schematic layouts of the shielding area. The $2.9 \mathrm{~m}$ high shield is a poured slab $2 \mathrm{~m}$ thick on top of $0.9 \mathrm{~m}$ tall shielding blocks. At $0^{\circ}, 22^{\circ}, 45^{\circ}$, and $90^{\circ}$, two meter long, $21.9 \mathrm{~cm}$ outside diameter PVC pipes are embedded vertically with centers located at depths given in Table 2.1. A concrete roof covers the shielding tunnel to minimize sky-shine neutron effects and to provide personnel protection.

During measurements, detectors are sunk into the PVC pipes to a depth of $1.9 \mathrm{~m}$ so their centers are at beam height. Concrete plugs are placed above the detectors and in holes not in use to avoid shielding voids. The density of the plugs, $\left(2.22 \mathrm{~g} \cdot \mathrm{cm}^{-3}\right)$, is greater than the nominal shield density $\left(1.88 \mathrm{~g} \cdot \mathrm{cm}^{-3}\right)$, therefore, inclusion of the plug increases the mass thickness $\left(\mathrm{g} \cdot \mathrm{cm}^{-2}\right)$ between the detector and the target. This is partly offset by the low density of PVC $\left(1.3 \mathrm{~g} \cdot \mathrm{cm}^{-3}\right)$ and the small air gap surrounding the plug. The density variation from the nominal shield density caused by the PVC pipe and concrete plug are accounted for by computing the mean density across the concrete-plug-filled PVC pipe diameter, $\left(2.06 \mathrm{~g} \cdot \mathrm{cm}^{-3}\right)$, and using this value to determine the mass thickness between the detector and the target. Detector depths are then stated 
as the mass thickness into the shield, or effective depths for a shield of density $1.88 \mathrm{~g} \cdot \mathrm{cm}^{-3}$ by dividing the mass thickness by the nominal density.

\begin{tabular}{||c|c|c|c|c|c|}
\hline \multirow{2}{*}{ Angle } & \multicolumn{5}{|c|}{ Hole } \\
\cline { 2 - 6 } & 1 & 2 & 3 & 4 & 5 \\
\hline $0^{\circ}$ & 32.4 & 93.2 & 154 & 215 & 276 \\
$22^{\circ}$ & 31.3 & 81.9 & 153 & 215 & 275 \\
$45^{\circ}$ & 31.3 & 82.5 & 133 & 187 & \\
$90^{\circ}$ & 31.0 & 70.4 & 112 & 153 & \\
\hline
\end{tabular}

Table 2.1: Physical locations of centers of the measurement tubes. Distances are given in $\mathrm{cm}$ from the air-shield interface.

\subsubsection{Concrete Composition}

Shielding concrete composition was determined through $\mathrm{x}$-ray fluorescence spectrometry ${ }^{2}$ on core samples poured with the shield. The composition is listed in Table 2.2 along with the composition of ordinary concrete as listed by Patterson [83]. The density, determined to be $1.88 \mathrm{~g} \cdot \mathrm{cm}^{-3}$ through measurement of the core samples, is below the density of standard concrete $\left(2.3 \mathrm{~g} \cdot \mathrm{cm}^{-3}\right)[56,83]$, however, the isotopic composition is quite similar.

\subsection{Targets}

Three targets, $\mathrm{Al}, \mathrm{Fe}$, and $\mathrm{Pb}$, whose lengths exceed the proton range were used in this investigation. These elements are chosen since they are typically used in construction of accelerators and beamlines, and represent a large range in atomic

\footnotetext{
${ }^{2}$ Analysis performed by Construction Technology Laboratories Incorporated, Skokie, Illinois.
} 


\begin{tabular}{|c|c|c|}
\hline \multirow{3}{*}{ Element } & \multicolumn{2}{|c|}{ Density $\left(\frac{\text { atomis }}{\mathrm{cm}^{3}}\left(\times 10^{22}\right)\right)$} \\
\hline & Our & Ordinary \\
\hline & Concrete & Concrete \\
\hline Oxygen & 3.84 & 4.73 \\
\hline Hydrogen & 1.90 & 1.73 \\
\hline Silicon & 0.68 & 1.57 \\
\hline Calcintin & 0.36 & 0.26 \\
\hline Carbon & 0.33 & - \\
\hline Magnesium & 0.16 & 0.01 \\
\hline Aluminum & 0.16 & 0.17 \\
\hline Iron & 0.04 & 0.05 \\
\hline Potassium & 0.03 & 0.03 \\
\hline Sodium & 0.01 & 0.03 \\
\hline
\end{tabular}

Table 2.2: Composition of the concrete used in these measurements, $\rho=$ $1.88 \mathrm{~g} \cdot \mathrm{cm}^{-3}$, and ordinary concrete listed by Patterson $|83| \rho=2.3 \mathrm{~g} \cdot \mathrm{cm}^{-3}$.

number. Moreover, these targets are similar to those used in the calculations of Alsmiller [8] and Hagen [54].

Table 2.3 lists target thicknesses, ranges of $233 \mathrm{MeV}$ protons, and locations of the effective target center for each target. Targets are longer than the proton range to ensure that all protons are stopped in the target. Target diameters of $10.2 \mathrm{~cm}$ ensure interception of the entire bea.n.

Targets are held in place by a positioning stand which includes an ionization chamber in fixed geometry to monitor local neutron production. This large volume ion chamber (Exradin $\left.A 6^{3}\right)^{3}$ is located $20 \mathrm{~cm}$ directly below the target center.

Targets are positioned $\approx 1 \mathrm{~m}$ from the shield face and $\approx 1 \mathrm{~m}$ above ground

\footnotetext{
${ }^{3}$ Manufactured by Exradin, Lisle, Illinois.
} 


\begin{tabular}{|l|c|c|c||}
$\begin{array}{l}\text { Target } \\
\text { Material }\end{array}$ & $\begin{array}{c}\text { Proton } \\
\text { Range } \\
\left(\mathrm{g} \cdot \mathrm{cm}^{-2}\right)\end{array}$ & $\begin{array}{c}\text { Target } \\
\text { Length } \\
\left(\mathrm{g} \cdot \mathrm{cm}^{-2}\right)\end{array}$ & $\begin{array}{c}\text { Effective } \\
\text { Center } \\
\left(\mathrm{g} \cdot \mathrm{cm}^{-2}\right)\end{array}$ \\
$\mathrm{Al}$ & 42.9 & 53.4 & 17.8 \\
$\mathrm{Fe}$ & 48.0 & 60.5 & 19.7 \\
$\mathrm{~Pb}$ & 66.9 & 77.4 & 26.6 \\
\hline
\end{tabular}

Table 2.3: Target thicknesses used in this study and the range of $230 \mathrm{MeV}$ protons. Effective centers for $233 \mathrm{MeV}$ protons are given in $\mathrm{g} \cdot \mathrm{cm}^{-2}$ from the beam entrance. Ranges determined from Janni [67].

level with their effective neutron production centers located at the intersection point of the four angles. The shielding arrangenent assumes a point source for neutron production at the intersection of the four angles. Slowing protons, however, produce neutrons over their entire slowing down path-length. Since neutron production is energy dependent, the source is essentially a nonuniform line source.

To estimate the effective source center, neutron production as a function of depth into the $\mathrm{Al}, \mathrm{Fe}$ and $\mathrm{Pb}$ targets is determined for $50-250 \mathrm{MeV}$ protons from neutron yield data. Tesch [105] provides a table of neutron yield as a function of incident proton energy for thick target neutron production. The energy is used to determine the residual proton range via range-energy tables $[67 \mid$. Yield versus residual range data are fitted to a second order polynomial $Y$. The slope of this polynomial, $Y^{\prime}$, gives the number of neutrons produced per path-length interval at a given distance from the end of range. The mean depth of neutron 
production, or effective neutron production center, is then

$$
\bar{x}=\frac{\int_{0}^{R} x Y^{\prime} d x}{\int_{0}^{R} Y^{\prime} d x},
$$

where $R$ is the proton range at the energy of interest in the material of interest, $x$ is the residual range, and $\bar{x}$ is the distance to end of range for the effective neutron source. Therefore, the effective target center is $R-\bar{x}$ from the target entrance. The effective target centers determined are given in Table 2.3 and are all $\approx 40 \%$ of the proton range.

\subsection{Tissue-Equivalent Proportional Counters}

The radiation environment produced as the protons interact with the target material is dominated by neutrons. As the radiation quality for neutrons varies significantly with energy, neutron energy information or mean radiation quality is needed to determine the dose equivalent deposited by the radiation. There are no detector systems suitable for determination of the entire neutron energy spectrum at the beam intensities available. Activation methods lack the required sensitivity to measure the neulron fluence at depth in the concrete shiald. Time-of-flight techniques require a pulsed beam and a low scatter environment, contrary to shielding geometries.

Measurement of absorbed dose through determination of the energy deposited by charged particles produced in neutron interactions with matter is quite straightforward using a low pressure tissue-equivalent proportional counter (TEP(!) [63]. Since events occurring in a TEPC are proportional to the quality of the radiation 
producing the event [63], mean quality factors and dose equivalent can also be determined from the data. ICRU 36 recommends the use of proportional counters for the measurement of absorbed dose deposited by high energy neutrons [6.3].

\subsubsection{Principles of Operation}

Low pressure tissue-equivalent proportional counters were used to measure the neutron and gamma components of the absorbed dose and dose equivalent at depth in the concrete shield. These counters operate via interactions in the A150 [97] wall material and surrounding material that produce charged particles which cross and ionize the counter gas. The electric field in the counter collects the ions produced. Near the center collection electrode, uniform charge multiplication occurs. The total charge collected after a single event is proportional to the primary ionization produced as the charged particle crosses the gas. Therefore, the electrical signal output is proportional to the total ionization produced by a single event. The electronic processing of this signal is described in detail in Section 2.5.4.

Two different spherical counter geometries are used. In the Rossi design (Figure 2.4) a central helix at $80 \%$ of the central collection electrode potential provides a uniform field gradient along the central collection electrode. Charge multiplication occurs in cylindrical geometry in the uniformly increasing electric field between the helix and the central electrode. The diameters of the Rossitype counters used are $12.5 \mathrm{~mm}$ and $56.9 \mathrm{~mm}$. Single wire proportional counters with diameters of $125.5 \mathrm{~mm}$ are also used and are shown in Figure 2.5. In these 


\begin{tabular}{|l|c|c|c|c||}
\hline Weight Percent & \multicolumn{4}{|c|}{ Element } \\
\cline { 2 - 5 } & $\mathrm{H}$ & $\mathrm{C}$ & $\mathrm{N}$ & 0 \\
\hline Srdoc Mixture & 10.3 & 56.9 & 3.5 & 29.3 \\
Present Mixture & 10.1 & 56.6 & 3.3 & 30.0 \\
\hline
\end{tabular}

Table 2.4: Composition of TE-propane gas mixture and comparison with mixture suggested by Srdoc [99]. Analysis by Matheson Gas Products Inc., Joliet, Illinois.

counters, the ratio of the counter to wire diameter is sufficient to provide a uniform electric field gradient for charge multiplication.

The counters are filled with the propane based tissue-equivalent gas mixture listed in Table 2.4 [99]. Gas filling procedures include evacuating the chambers to less than $\approx 0.05$ Torr for a period of a few hours to eliminate residual debris, then filling the chamber to $\approx 100$ Torr and re-evacuating to $\approx 5$ Torr. This procedure is repeated three times to flush the chamber before a final fill to $\approx 100$ Torr and subsequent evacuation to the desired gas pressure.

(ias pressures are chosen such that the energy lost by a particle crossing the gas cavity of the chamber is the same as for the particle crossing $2-4 \mu \mathrm{m}$ of unit density tissue. The energy lost by a particle crossing the gas is

$$
\Delta E_{g}=\left.\frac{d E}{\rho d x}\right|_{g} \rho_{g} \Delta x_{g}
$$

and the energy lost by the particle as it crosses tissue is

$$
\Delta E_{t}=\left.\frac{d E}{\rho d x}\right|_{t} \rho_{t} \Delta x_{t} .
$$

where $\frac{d E}{\partial d x}$ represents the stopping power, $\rho$ represents the density, $\Delta x$ represents the charged particle path-length in the gas and the subscripts $g$ and $t$ represent. 
$\bigotimes$ Aluminum

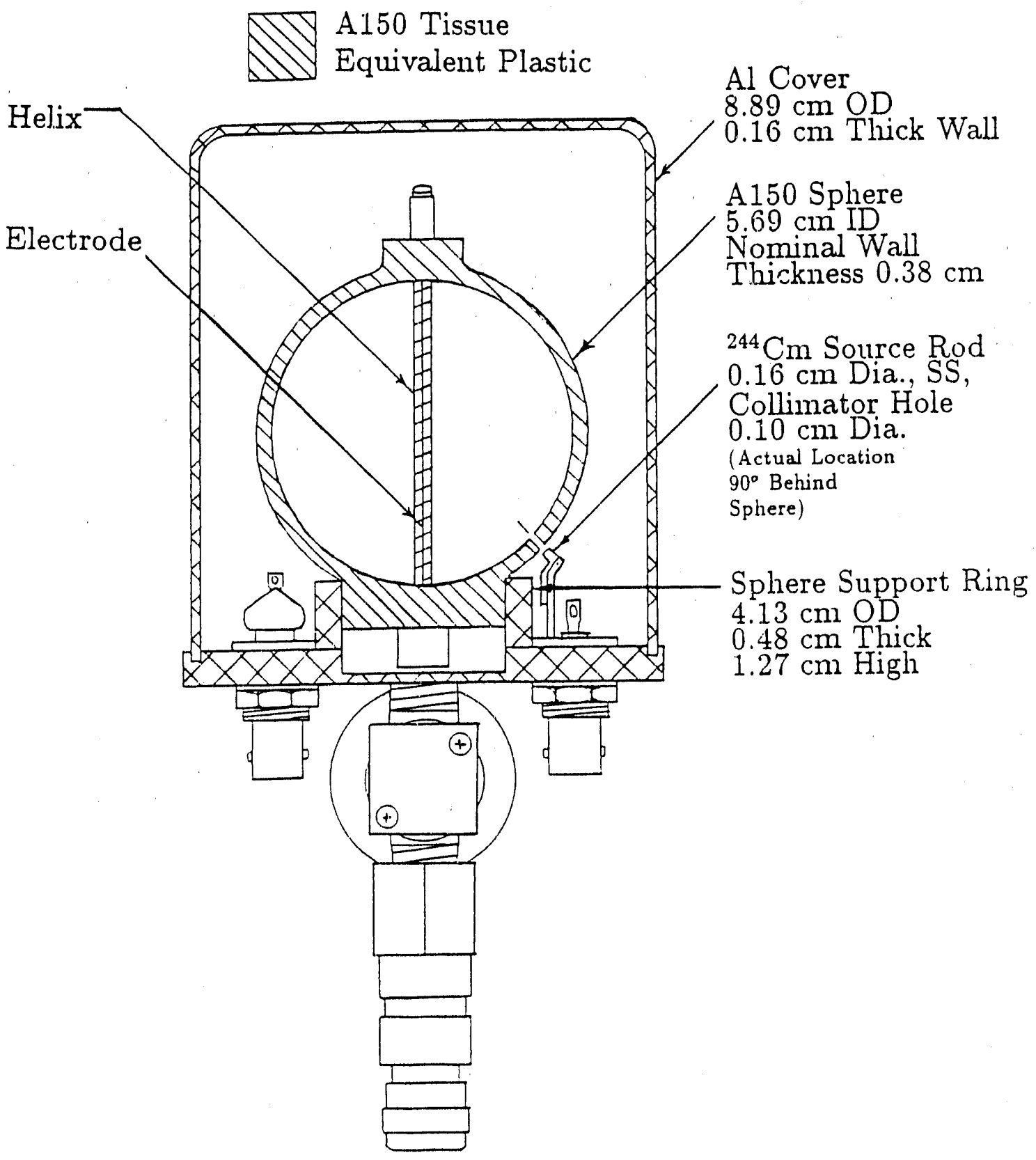

Figure 2.4: Schematic drawing of the Rossi type tissue-equivalent proportional counter used in these measurements. The central helix is at an intermediate potential, therefore ion multiplication occurs in cylindrical geometry in the region surrounding the central wire. (Manufactured by Far West Technologies. Cioleta ('A). 


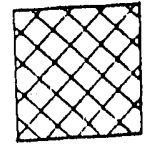

Aluminum

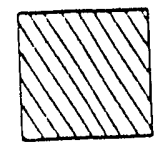

A150 Tissue

Equivalent Plastic

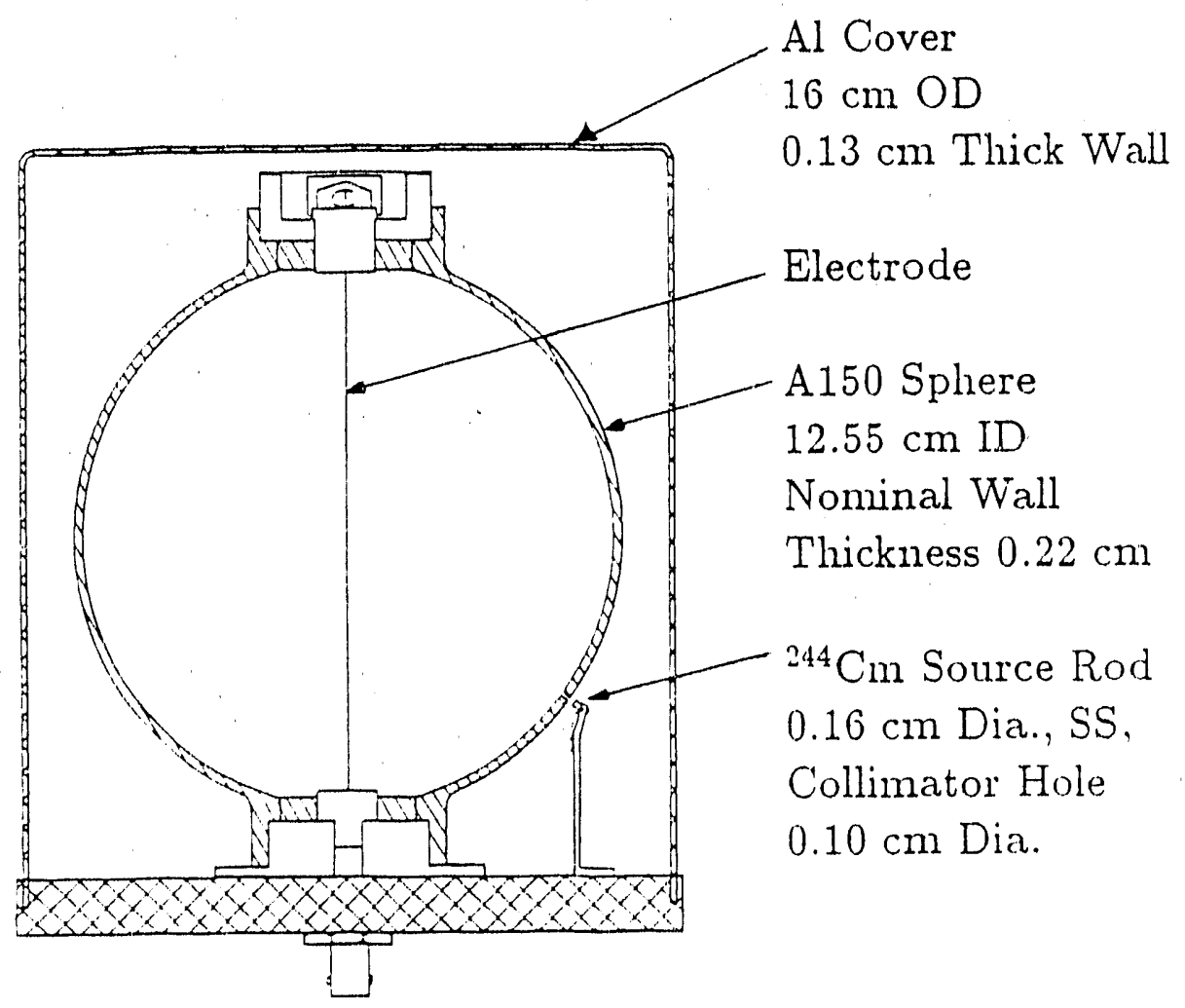

Figure 2.5: Illustration of the single wire tissue-equivalent proportional connters used in these measurements. Counters have Also tissue-equivalent walls and are filled wit': tissue-equivalent propane kas. Manufactuled liy Far West Technologies, Cioleta ( A ). 
the gas and tissue respectively. The resulting gas density for equal energy loss is then

$$
\rho_{g}=\rho_{t} \Delta x_{g}^{t} S_{g}^{t}
$$

where $\Delta x_{g}^{t}$ represents the ratio of the path-length in tissue to that in the chamber and ${ }_{m} S_{g}^{t}$ represents the ratio of the tissue to gas stopping powers. Using the fact that

$$
\rho_{g}=\rho_{g_{S T P}} P_{g_{S T P}}^{P_{g}},
$$

where $P_{g_{S T P}}$ and $\rho_{g_{S T P}}$ are the gas pressure and density under standard conditions and $P_{g}$ is the desired gas pressure, the resultant gas pressure is

$$
P_{g}=P_{g_{S T P}} \frac{\rho_{t}}{\rho_{g_{S T P}}} \Delta x_{g m}^{t} S_{g}^{t}
$$

The gas pressures used are listed in Table 2.5 along, with the tissue-equivalent thickness of the chamber at the stated pressure for each chamber size used. The propane gas mixture was chosen due to its high gas gain and stability $[40,42]$.

\begin{tabular}{|l|c|c|}
\hline $\begin{array}{l}\text { Detector } \\
\text { Diameter } \\
(\mathrm{mm})\end{array}$ & $\begin{array}{c}\text { Gas } \\
\text { Pressure } \\
\text { (Torr) }\end{array}$ & $\begin{array}{c}\text { Tissue } \\
\text { Equivalent } \\
\text { Thickness } \\
\left(\mu \mathrm{m}_{\text {tissue }}\right)\end{array}$ \\
\hline 12.5 & 120 & 4 \\
56.9 & 12 & 2 \\
125.5 & 12 & 4 \\
\hline
\end{tabular}

Table 2.5: Gas pressures and corresponding tissue-equivalent chamber thickness for each chamber and gas pressure used. 


\subsubsection{Cavity Theory for Low Pressure TEPC}

The energy deposited in the gas of a tissue-equivalent proportional counters is related to the absorbed dose in tissue through the use of cavity theory. First, it is important to understand the steps involved in the energy deposition. Indirectly ionizing radiations, neutrons for example, deposit dose in material in a two-step process. Neutrons transfer energy to atomic nuclei, then these secondary charged particles dissipate the transferred energy by undergoing further electronic collisions [61]. As the charged secondaries have finite ranges in matter, the two stages of energy deposition may take place at different points in the medium. Energy transferred to charged particles by the indirectly ionizing radiation (kerma) is then deposited (dose) in a surrounding location. Tissue-equivalent proportional counters measure a charge proportional to the energy deposited in the gas by secondary charged particles that cross it. This energy deposited can be related to absorbed dose in tissue when the fluence of charged particles in the immediate vicinity of the counter is constant, that is, charged particle equilibrium (CPE) exists $[20,61,63]$.

The proportional counters used in these measurements are constructed from tissue-equivalent plastic, A150, and are filled with tissue-equivalent gas, TEpropane. The Fano theorem states that in a medium of constant atomic composition exposed to a uniform field of indirectly ionizing radiation, the fluence of secondary particles is constant and independent of the density of the medium, provided that the interaction cross sections and stopping powers of the particles are independent of density $[20,47,63]$. Since the wall and gas in the proportional 
counter are of similar atomic composition, CPE exists for all charged particles whose ranges are less than the proportional counter wall thickness. For these secondaries, the dose deposited in the tissue-equivalent counter is equal to the dose that would be deposited in tissue at the counter location. Unfortunately, secondaries are produced with ranges greater than the wall thickness.

Neutrons can be produced with energies up to that of the inciclent proton beam, $230 \mathrm{MeV}$. These, in turn, can produce $230 \mathrm{MeV}$ protons whose range is $35 \mathrm{~cm}$ of tissue-equivalent material. Clearly, for these high energy particles, CPE is not achieved in either the detector, or most humans at the detector location.

Inadequacies of the wall thickness being less than the range of the charged particles produced is compensated by the niaterials surrounding the chamber. Figure 2.6 shows a schematic layout of the surrounding materials. For neutrons, the tissue equivalence of materials is determined primarily by the hydrogen content of the material. Table 2.6 lists the hydrogen mass thickness of A-150 plastic as well as other materials surrounding the chamber. The Lucite counter holder provides an additional $0.635 \mathrm{~cm}$ of plastic (approximately tissue-equivalent) around the chamber, and the PVC pipe cast into the shield adds another $0.903 \mathrm{~cm}$ of plastic. Additionally, the concrete shielding itself is fairly tissue-equivalent. Finally, the most probable energy neutron or proton energy following a $(p, n)$ or $(n, p)$ interaction is less than half of the maximum energy. A minimum of two of these interactions occurs for an event to be observed in a TEPC, one in the target. producing the neutron and one near the counter producing a charged particle. Thus, the most probable secondary range is less than the range of a $60 \mathrm{MeV}$ 
proton, $3.1 \mathrm{~g} \cdot \mathrm{cm}^{-2}$. Therefore, the effects due to the counter wall being less than the maximum range of the secondary charged particles produced should be minimal and the close deposited in the tissue-equivalent counter should closely approximate the dose that would be deposited in tissue at the counter location.

\begin{tabular}{|c|c|c|c|c|}
\hline \multirow[b]{2}{*}{ Material } & \multicolumn{2}{|c|}{$56.9 \mathrm{~mm}$ Counter } & \multicolumn{2}{|c|}{$125.5 \mathrm{~mm}$ Counter } \\
\hline & $\begin{array}{l}\text { Thickness } \\
(\mathrm{cm})\end{array}$ & $\begin{array}{l}\text { Hydrogen Content } \\
\qquad\left(\mathrm{g} \cdot \mathrm{cm}^{-2}\right)\end{array}$ & $\begin{array}{c}\text { Thickness } \\
(\mathrm{cm})\end{array}$ & $\begin{array}{l}\text { Hydrogen Content } \\
\left(\mathrm{g} \cdot \mathrm{cm}^{-2}\right)\end{array}$ \\
\hline $\mathrm{A} 150$ & 0.38 & 0.0434 & 0.22 & 0.0251 \\
\hline Lucite & - & - & 0.64 & 0.0609 \\
\hline PVC & 0.47 & 0.0296 & - & - \\
\hline PVC & 0.90 & 0.0568 & 0.90 & 0.0568 \\
\hline Total & 1.75 & 0.1298 & 1.76 & 0.1428 \\
\hline
\end{tabular}

Table 2.6: Thicknesses and hydrogen mass thicknesses of materials surrounding the proportional counters.

\subsubsection{Detector Holders}

A detector holder is used to bear the weight of the concrete plugs placed above the detector and to protect the detector from damage and debris. Since neutron energies can approach the primary beam energy of $230 \mathrm{MeV}$, the detector holders also provide extra material to provide charged particle equilibrium for the neutrons. For the $56.9 \mathrm{~mm}$ counters, the holder is constructed from a PVC pipe with a $4.7 \mathrm{~mm}$ thick wall. For $125.5 \mathrm{~mm}$ counters, $6.35 \mathrm{~mm}$ thick Lucite pipes are used. Lucite caps are placed on each end of the pipe to hold the retectors in place and to keep dirt and debris from the detector. Detector cabling runs along the side of the pipes. For increased rigidity in rase a concrete plug is dropped 


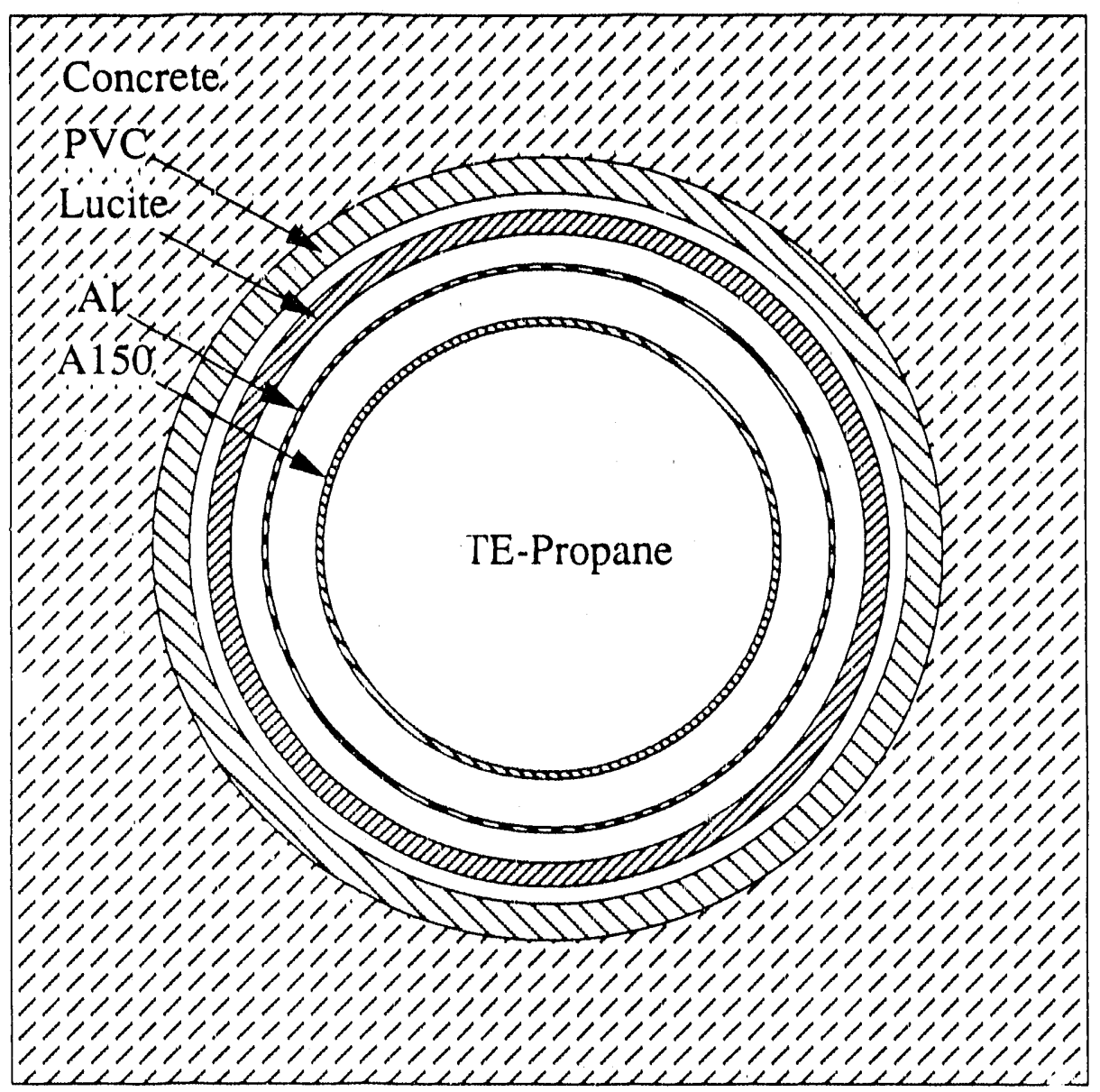

Figure 2.6: Cross-sectional view of materials surrounding the tissue-equivalent proportional counters. Material thicknesses are listed in Table 2.6. 
on the detector holder, strips of glass epoxy are glued to the outside protruding $2 \mathrm{~cm}$ from the ends of the pipe. Since the inner diameter from the glass epoxy stripes is less than the outer diameter of the concrete plugs, the plugs rest on these strips. Ropes are attached to the detector holders for removal of detectors from the holes after use. Cables for the detectors are stress relieved at the detector holders.

\subsubsection{TEPC Electronics}

Neutron interactions in the material surrounding a tissue-equivalent proportional counter produce charged particles which cross and ionize the gas. The charge produced is collected by the central electrode and is amplified by factors of $10^{3}-10^{4}$ by charge multiplication in the gas $[42,43]$.

A charge sensitive preamplifier converts the charge signal to a voltage signal suitable for transmission through long cable lengths. Signals from each preamplifier travel through $30 \mathrm{~m}$ of $\mathrm{RG}-58$ coaxial cable to a pair of linear spectroscopy amplifiers. Each detector uses two linear amplifiers set at gains differing by a factor of 30 to record the entire event spectrum in two sections. This is necessary since the event sizes observed vary over four orders of magnitude.

Amplifier outputs are connected to 1024 channel analog-to-digital converters (ADCs). Digitized events trip an interrupt in a local computer, which reads in the digitized event size through a parallel input-output unit. Alter servicing an event, the computer clears the $\mathrm{ADC}$ and allows it to accept further events. The computer stores and processes the event size data. One proportional counter 
has its signal lines routed into a pair of multichannel analyzers which stored the events. Following data collection, these event spectra were read into a computer through a serial line for long-term storage and analysis.

To minimize background events, the entire data aquisition system is gated on only during beam extraction using a trigger pulse available from the accelerator control system. Figure 2.7 shows a schematic layout of the electronics for a single proportional counter. The interconnections between all of the counters and the beam monitoring devices is shown in Figure 2.8 .

Scalers which recorded the transmission ion chamber signals are also gated to ensure currect normalization.

\subsubsection{Data Collection Procedures-Statistics}

Data are collected using three TEPC's simultancously. Each detector is lowered into a detector hole at a different angle in the concrete shield. Concrete plugs are placed above the detectors to prevent neutron leakage from above and are also placed in holes not in use so a solid shield exists.

Each TEPC is then calibrated using methods outlined in Section 2.5.6. Following verification that the proton beam is striking the target, data aquisition begins.

A large range of run times and cose rates was enconintered during the data collection process. A few statistics on these quantities are summarized in Table 2.7.

Since the accelerator spill lasted less than one second, with an eight second 


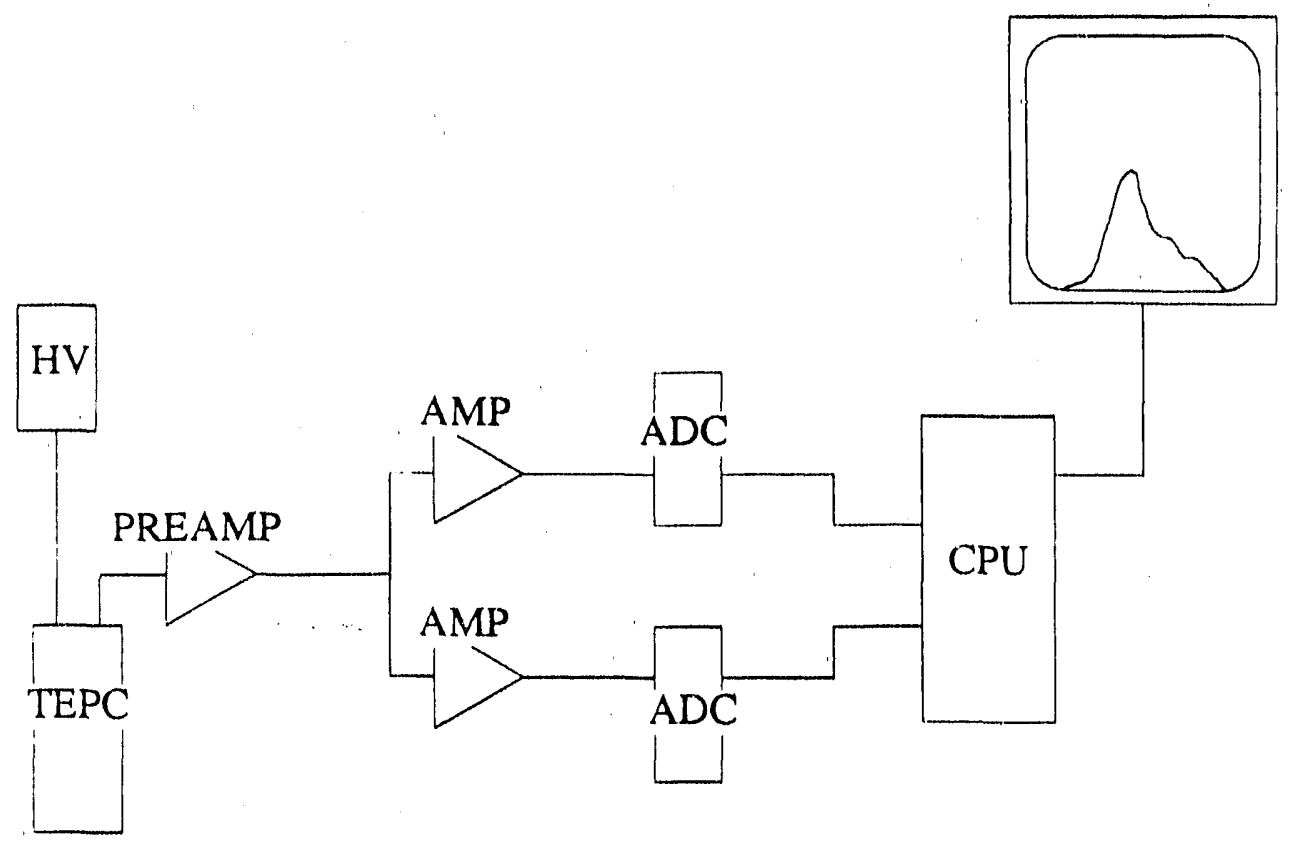

Figure 2.7: Schematic diagram of the electrical layout for one TEPC!. The preanplifier signal is fed into a pair of linear amplifiers set at different gains to observe the entire event size spectrum. 


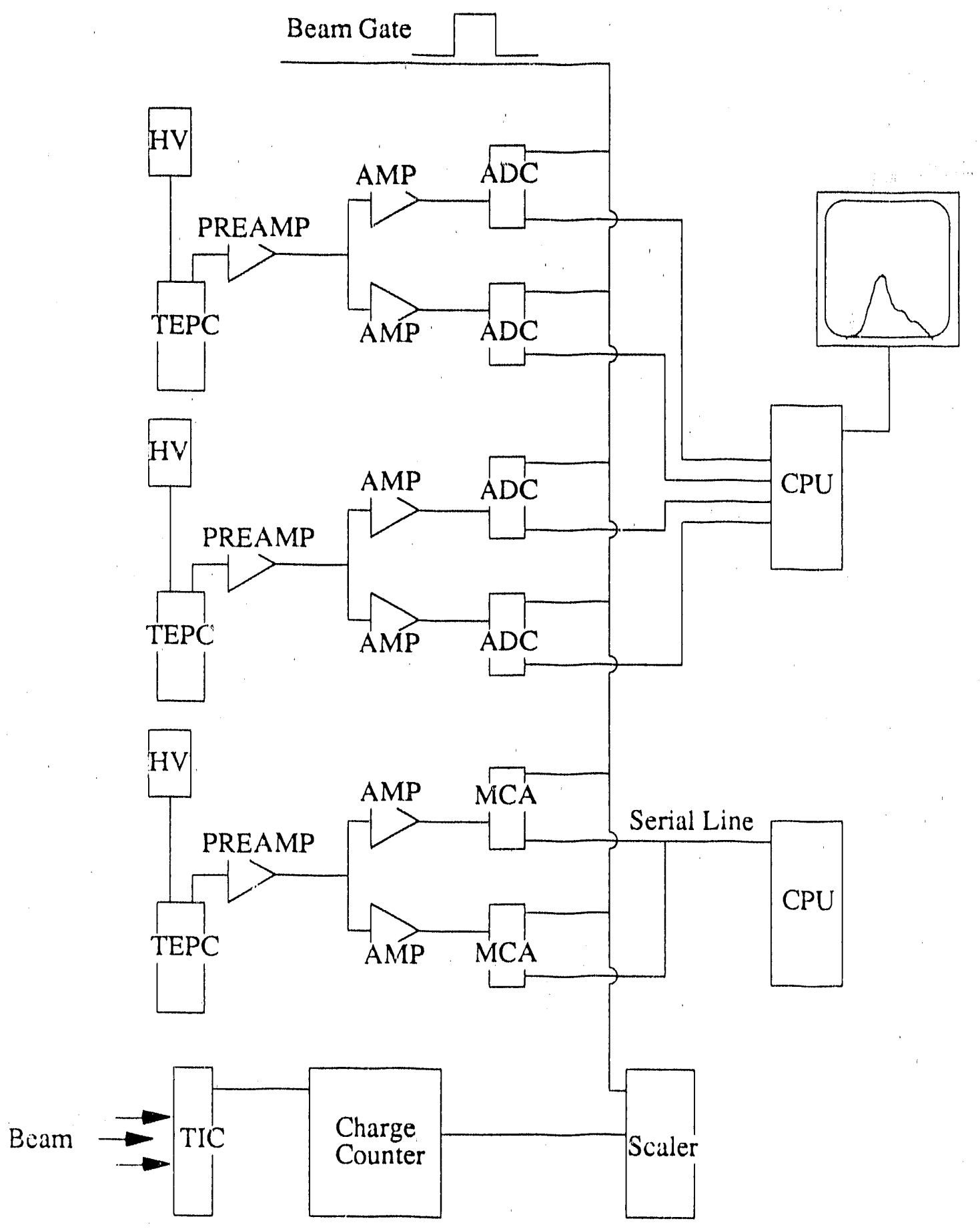

Figure 2.8: Schematic diagram of the inter-connections of 'TEP(' ind beam monitoring electronics. All electronics are gated to ensure only beim indured rents are recorded. Only one ' $T$ ' $C$ ' is pictured, however a minimum of f wo were in the beam at all times. 


\begin{tabular}{|c|c|c|c|}
\hline Quantity & $\begin{array}{l}\text { Maximum } \\
\text { Value }\end{array}$ & $\begin{array}{l}\text { Minimum } \\
\text { Value }\end{array}$ & $\begin{array}{c}\text { Average } \\
\text { Value }\end{array}$ \\
\hline Run time & 10 hours & $20 \mathrm{~min}$ & 2.9 hours \\
\hline Live Time & $4450 \mathrm{sec}$ & $90 \mathrm{sec}$ & $1150 \mathrm{sec}$ \\
\hline Dose Deposited & $13 \cdot \mathrm{mSv}$ & $1 \mu \mathrm{Sv}$ & $0.9 \mathrm{mS} \mathrm{v}$ \\
\hline Dose Rate & $0.17 \mathrm{~Sv} \cdot h r^{-1}$ & $3.25 \mu \mathrm{Sv} \cdot \ln \mathrm{r}^{-1}$ & $6.75 \mathrm{mSv} \cdot \mathrm{hr}^{-1}$ \\
\hline Protons per run & $2.7 \cdot 10^{13}$ protons & $6.4 \cdot 10^{11}$ protons & $4.8 \cdot 10^{12}$ protons \\
\hline
\end{tabular}

Table 2.7: Maximum, minimum, and average values of run-time quantities during data collection.

repetition rate, run times ranging from 20 min to $10 \mathrm{hr}$ resulted in counter live times of 90 to $4450 \mathrm{~s}$.

A total of $1.0 \cdot 10^{14}$ protons were incident upon three targets, distributed nearly equally with each receiving approximately $3.3 \cdot 10^{13}$ protons.

Multiple runs were often taken for a given target, angle, depth combination. A total of 171 two gain event size spectra were measured using tissue-equivalent proportional counters, 156 in-shield measurements and 15 measurements in air using a $12.5 \mathrm{~mm}$ diameter TEPC. Table 2.8 shows how the in-shield measurements were divided among targets, depths, and angles.

\subsubsection{Calibration of Low Pressure TEPC}

TEPC events are recorded by an analog-to-digital converter and stored in a channel that is proportional to the number of ion pairs produced as a charged particle crosses the counter. Conversion of the channel number to the energy loss by charged particles crossing the gas requires calibration of the TEPC!

If both the electronic and gas gain of the proportional counter are considereri, 


\begin{tabular}{|c|c|c|c|c|}
\hline \multirow[t]{2}{*}{ Depth } & \multicolumn{4}{|c|}{ Angle } \\
\hline & $0^{\circ}$ & $22^{\circ}$ & $45^{\circ}$ & $90^{\circ}$ \\
\hline \multirow{3}{*}{1} & $\mathrm{Al}(\mathrm{A}: 2)$ & $\mathrm{Al}(\mathrm{A}: 4, \mathrm{D}: 1)$ & $\operatorname{Al}(A: 2, D: 2)$ & $\mathrm{Al}(\mathrm{A}: 3, \mathrm{~B}: 1)$ \\
\hline & $\mathrm{Fe}(\mathrm{A}: 3, \mathrm{D}: 2)$ & $\mathrm{Fe}(\mathrm{A}: 6)$ & $\mathrm{Fe}(\mathrm{A}: 2, \mathrm{D}: 3)$ & $\mathrm{Fe}(\mathrm{A}: 3, \mathrm{~B}: 3)$ \\
\hline & $\mathrm{Pb}(\mathrm{A}: 3)$ & $\mathrm{Pb}(\mathrm{A}: 3, \mathrm{D}: 3)$ & $\mathrm{Pb}(\mathrm{A}: 2, \mathrm{D}: 2)$ & $\mathrm{Pb}(\mathrm{A}: 2)$ \\
\hline \multirow{3}{*}{2} & $\mathrm{Al}(\mathrm{A}: 3)$ & $\overline{\mathrm{Al}}(\mathrm{A}: 1, \overline{\mathrm{C}: 1, \mathrm{D}: 1)}$ & $\mathrm{Al}(\mathrm{B}: 1, \mathrm{C}: 1, \mathrm{D}: 1)$ & $\mathrm{Al}(\mathrm{B}: 2)$ \\
\hline & $\mathrm{Fe}(\mathrm{A}: 4)$ & $\mathrm{Fe}(\mathrm{A}: 2, \mathrm{C}: 2)$ & $\mathrm{Fe}(\mathrm{A}: 1, \mathrm{~B}: 1, \mathrm{C}: 1)$ & $\operatorname{Fe}(A: 1, B: 3)$ \\
\hline & $\mathrm{Pb}(\mathrm{A}: 1)$ & $\mathrm{Pb}(\mathrm{A}: 1, \mathrm{D}: 1)$ & $\mathrm{Pb}(\mathrm{A}: 2)$ & $\mathrm{Pb}(\mathrm{B}: 1)$ \\
\hline \multirow{3}{*}{3} & $\mathrm{Al}(\mathrm{A}: 2, \mathrm{C}: 1)$ & $\mathrm{Al}(\mathrm{A}: 1, \mathrm{~B}: 1, \mathrm{C}: 3)$ & $\overline{\mathrm{Al}(\mathrm{C}: 1)}$ & $\mathrm{Al}(\mathrm{B}: 3)$ \\
\hline & $\mathrm{Fe}(\mathrm{A}: 3, \mathrm{C}: 2)$ & $\operatorname{Fe}(\mathrm{B}: 1, \mathrm{C}: 2)$ & $\mathrm{Fe}(\mathrm{C}: 1)$ & $\mathrm{Fe}(\mathrm{B}: 4, \mathrm{C}: 1)$ \\
\hline & $\mathrm{Pb}(\mathrm{A}: 1, \mathrm{C}: 2)$ & $\mathrm{Pb}(\mathrm{A}: 1, \mathrm{~B}: 2, \mathrm{C}: 2)$ & $\mathrm{Pb}(\mathrm{C}: 1)$ & $\mathrm{Pb}(\mathrm{B}: 1)$ \\
\hline \multirow{3}{*}{4} & $\mathrm{Al}(\mathrm{B}: 1, \mathrm{C}: 3)$ & $\overline{\mathrm{Al}}(\mathrm{C}: 1)$ & $\mathrm{Al}(\mathrm{B}: 1)$ & $\mathrm{Al}(\mathrm{B}: 3)$ \\
\hline & $\mathrm{Fe}(\mathrm{C}: 4)$ & $\mathrm{Fe}(\mathrm{B}: 2, \mathrm{C}: 2)$ & $\mathrm{Fe}(\mathrm{B}: 2)$ & $\mathrm{Fe}(\mathrm{B}: 1)$ \\
\hline & $\mathrm{Pb}(\mathrm{A}: 1, \mathrm{C}: 1)$ & $\mathrm{Pb}(\mathrm{B}: 1, \mathrm{C}: 1)$ & $\mathrm{Pb}(\mathrm{B}: 2, \mathrm{C}: 1)$ & $\mathrm{Pb}(\mathrm{B}: 2)$ \\
\hline \multirow{3}{*}{5} & $\mathrm{Al}(\mathrm{C}: 1)$ & $\mathrm{Al}(\mathrm{B}: 1)$ & & \\
\hline & $\mathrm{F} \in(\mathrm{C}: 1)$ & & & $\mathrm{Fe}(\mathrm{C}: 1)$ \\
\hline & $\mathrm{Pb}(\mathrm{B}: 1)$ & & & $\mathrm{Pb}(\mathrm{C}: 2)$ \\
\hline
\end{tabular}

Table 2.8: Measurements made using TEPC's for each target material at each depth and angle. Depths are given as hole number and can be related to concrete thickness using Table 2.1. Read $\mathrm{Fe}(\mathrm{A}: 1, \mathrm{~B}: 2, \mathrm{C}: 3)$ as Fe target, one measurement with counter $A, 2$ measurements with counter $B$, and 3 measurements with counter C. Counter A: $57.2 \mathrm{~mm}$ Rossi Type, SN: 199. Counter B: $125.5 \mathrm{~mm}$ Single Wire, SN: 1174, Counter C: $125.5 \mathrm{~mm}$ Single Wire, SN: 214, Counter D: $57.2 \mathrm{~mm}$ Rossi Type, SN: 175. All counters manufactured by Far West Technologies, Goleta, CA. 
for a charged particle of type $x$ with energy $E$, the event size observed will be

$$
S[\text { volts }]=\left.\Delta E G_{e} G_{g} C \frac{e}{\bar{W}}\right|_{x}
$$

where $S$ is the pulse height observed in volts that is digitized by the ADC, $\triangle E$ is the energy lost by the charged particle as it crosses the proportional counter cavity, $\left.\stackrel{W}{e}\right|_{x}$ is the mean energy required to produce an ion pair in the cavity gas, $G_{e}$ and $G_{g}$ are the electronic and gas gains respectively, and $C$ is a constant used to correct units.

In calibrating the counter, instead of determining the $G_{e}$ and $G_{g}$, the product $\left.\frac{e}{W}\right|_{x} G_{e} G_{g}$ is determined using an internal ${ }^{244} \mathrm{C} / \mathrm{m}$ alpha particle source that is mounted behind a magnetically controlled shutter.

${ }^{244} \mathrm{Cm}$ emits two alpha particles with energies of $5.805 \mathrm{MeV}(76.7 \%)$ and 5.763 $\mathrm{MeV}(23.3 \%)$ [69]. Using the mean stopping power of ${ }^{244} \mathrm{C} m$ alpha particles along the known path-length traversed, the energy calibration of the counter is accomplished. Due to source encapsulation, the energy of the alphas as they enter the gas cavity is less than the energy at emission. Based on the manufacturers specified encapsulation thickness of $0.2 \mathrm{mg} \cdot \mathrm{cm}^{-2}$ of $\mathrm{Au}[76]$, an alpha particle would lose approximately $42 \mathrm{keV}$. Measurements by Menzel et al. indicate an average observed energy loss of about $375 \mathrm{keV}$, suggesting an encapsulation thicknesses of $1.86 \mathrm{mg} \cdot \mathrm{cm}^{-2}[76]$. The source thickness is ignored since it causes less than $1 \mathrm{keV}$ of energy loss.

Using the alpha particle energy entering the gas cavity, the mean alpha stopping power is calculated using the data of Anderson and Zeigler $\{10\}$. To account. for the increase in stopping power as the alpha crosses the cavity due to de- 


\begin{tabular}{|c|c|c|}
$\begin{array}{c}\text { Counter } \\
\text { Simulated } \\
\text { Diameter } \\
(\mu \mathrm{m} \text { tissue })\end{array}$ & $\begin{array}{c}0.2 \mathrm{mg} \cdot \mathrm{cm}^{-2} \mathrm{Au} \\
\text { Encapsulation } \\
\text { Mean Event Size } \\
\left(\mathrm{keV} \cdot \mu \mathrm{m}^{-1}\right)\end{array}$ & $\begin{array}{c}1.86 \mathrm{mg} \cdot \mathrm{cm}^{-2} \mathrm{Au} \\
\text { Encapsulation } \\
\text { Mean Event Size } \\
\left(\mathrm{keV} \cdot \mu \mathrm{m}^{-1}\right)\end{array}$ \\
\hline 1.0 & 83.74 & 87.92 \\
2.0 & 84.18 & 88.12 \\
4.0 & 85.42 & 89.16 \\
10.0 & 88.05 & 92.63 \\
\hline
\end{tabular}

Table 2.9: Alpha particle event sizes for several different simulated tissue diameters. Values given for source encapsulation thicknesses as stated by manufacturer $\left(0.2 \mathrm{mg} \cdot \mathrm{cm}^{-2}\right.$ of $\left.\mathrm{Au}\right)$ and as measured by Menzel $\left(1.86 \mathrm{mg} \cdot \mathrm{cm}^{-2}\right.$ of $\left.\mathrm{Au}\right)[76]$.

creasing alpha energy, the cavity is divided into 1000 equal intervals, and after each interval, the alpha energy and stopping power is recomputed. The total energy loss by an alpha crossing the chamber diameter divided by the chamber simulated diameter is then computed. This is done for each initial alpha energy, and the values added, weighted by the initial alpha emission probability, to determine the average alpha event size. Table 2.9 lists the mean alpha event size as a function of simulated diameter of the proportional counter using the two different source encapsulation thicknesses. For the simulated diameters used in our measurements, 2 and $4 \mu \mathrm{m}$, the mean alpha event size, $y_{\alpha}$, is taken to be $88.2 \mathrm{keV} \cdot \mu \mathrm{m}^{-1}$.

TEPC's measure a charge proportional to the number of ion pairs formed as a charged particle crosses the counter volume. However, the quantity of interest. in dosimetry is the energy imparted to a specific mass so absorbed dose can be calculated. The charge and energy imparted are related by the energy expended per ion pair formed in the gas $(W)$. Since $W$ varies as a function of both particle 
type and energy, precise evaluation of the energy imparted to the gas requires evaluation of $W$ for each particle at its mean energy as it crosses the gas. This procedure is not possible due to the multiple combinations of particle types and energies which can produce any given event size. Therefore, a mean $\bar{W}$ value must be estimated. Fortunately $W$ is largely energy independent and the $W$ of $5 \mathrm{MeV}$ alpha particles used in the calibration does not differ significantly from that of protons $\{62\}$. Therefore, the alpha calibration is reasonably precise for spectra dominated by recoil protons and alpha particles [63]. Since our spectra meet these criteria, no $\bar{W}$ value corrections were made.

Calibration runs which included the measurement of alpha event size spectrum and a precision pulser spectrum are recorded before each run. The precision pulser linearizs the pulse height spectrum. A pulser signal is put into the preamplifier test input, the location (channel) of the pulse produced recorded, then the input signal divided by factors of $2,5,10,20,50$, etc. The resultant pulse height spectrum is linearized using a least squares fitting procedure to

$$
P=m \cdot H+b
$$

where $P$ is the pulse height, expressed as a ratio with respect to the full pulse height, (i.e. divide by $2=0.5$ ), $H$ is the channel where the pulser peak is observed, and $m$ and $b$ are the slope and intercepts of the fitted line respectively. The alpha peak is fit with a Gaussian, and the center location converted to an equivalent pulse height,

$$
P_{\mathrm{ax}}=m \cdot H_{\mathrm{a}}+b
$$

Event channel numbers are converted to event sizes $\left(\mathrm{keV} \cdot \mu \mathrm{m}^{-1}\right)$ by multiplying 
the slope and intercept values by the ratio of the alpha event size $\left(y_{\alpha}\right)$ to $P_{\alpha}$,

$$
\begin{aligned}
& \alpha=\frac{y_{\alpha}}{P_{\alpha}} \cdot b \\
& \beta=\frac{y_{\alpha}}{P_{\alpha}} \cdot m .
\end{aligned}
$$

The channel numbers are then converted to event sizes.

$$
y(H)=\alpha+\beta \cdot H \quad\left[\mathrm{keV} \cdot \mu \mathrm{m}^{-1}\right]
$$

\section{Dose Determination}

Absorbed dose is also determined using the alpha calibration procedure. The dose contribution from a single alpha event is

$$
d_{\alpha}=\underset{m_{\text {ga }} \rho d x}{1} \frac{\overline{d E}}{\rho d} \rho \Delta x .
$$

Noting that the mass of the gas is $m_{g a s}={ }_{3}^{4} \pi r^{3} \rho$ where $r$ is the counter radius, $\rho$ is the gas density, and that $\Delta x=2 r, d_{\alpha}$ simplifies to

$$
d_{\alpha}=\left.\frac{d E}{\rho d x}\right|_{\alpha} \frac{3}{2 \pi r^{2}}
$$

Assuming $\rho_{\text {tissue }}=1 \mathrm{~g} \cdot \mathrm{cm}^{-3}, \overline{d E} /\left.d x\right|_{\alpha}=y_{c x}$, and converting to dose units (gray) we get

$$
d_{\alpha}=\frac{3}{2 \pi r^{2}} y_{\alpha} 1.602 \cdot 10^{-9}
$$

Therefore, to convert from pulse height to dose, one must multiply an event size by

$$
k=\frac{3}{2 \pi r^{2}} 1.602 \cdot 10^{-9} \quad\left|G \mathrm{y} /\left(\mathrm{keV} \cdot \mu_{\mathrm{m}}^{-1}\right)\right|
$$


The total dose for the sum of events at any event size is then given by

$$
d(y)=k y N(y)
$$

where $N(y)$ is the number of events with size $y$. Dose for an entire spectrum of events is determined by

$$
D=k \cdot \sum_{y=y_{\min }}^{y \max }[y \cdot N(y)] \quad[\mathrm{G} y] .
$$

\section{Dose Equivalent Determination}

Dose equivalent calculations require knowledge of the quality factor for the radiation field of interest. Mean quality factors, $\bar{Q}$, are determined for each event size spectrum observed using relationships between the lineal energy and linear energy transfer recommended by the ICIRU [63].

$$
\bar{Q}=0.8+0.14 \bar{y}_{d}
$$

The mean lineal energy, $\bar{y}_{d}$, is the second moment of the event size frequency distribution,

$$
\bar{y}_{d}=\frac{\sum_{y=y_{\min }}^{y_{\max }} y^{2} N(y)}{\sum_{y=y_{\min }}^{y_{\max }} y N(y)} .
$$

Dose equivalent is determined by

$$
H=D \bar{Q}
$$

Mean quality factors are determined separately for each event size spectrum observed. Several of these values were compared with a more recent ICRU for- 
malism [64],

$$
Q(y)=\frac{5510}{y}\left[1-\exp \left(-5 \cdot 10^{-5} y^{2}-2 \cdot 10^{-7} y^{3}\right)\right]
$$

and the values were found to agree to within $2 \%$ of the values determined using Equation 2.19.

\subsubsection{Error Analysis in Dose and Dose Equivalent Determination}

Absorbed dose and quality factor values determined have associated uncertainties due to the statistical fluctuation in events of the composite event size spectrum. In this error analysis, each event size is treated as a separate measurement. Due to the low probability of occurrence for each event size, Poisson statistics are employed. Therefore, at event size $y$ where $N$ events are observed during the counting interval, the probable error in $N$ is $\sqrt{N}$.

Additional errors are associated with alpha calibration due to uncertainty of the alpha peak position and uncertainty in the alpha event size. The uncertainty in the alpha event size is due to the uncertainty in the gas pressure as well as the source encapsulation thickness (Section 2.5.6). Uncertainty in the gas pressure only affects the evaluation of the mean alpha event size. Since this is a slowly varying function of gas pressure, it has minimal effect. In all, the alpha particle calibration uncertainty is about $3 \%$.

Full evaluation of systematic errors are not included in this error evaluation. A result of this is that the absolute errors are likely to be larger than the stated errors. However, the relative errors are believed to be correct.

To evaluate the uncertainty in absorbed dose, dose equivalent, and radiation 
quality values requires propagation of the uncertainties for each event size. The uncertainty in a function $q=\mathrm{f}(u, v)$, where $u$ and $v$ are measured quantities with associated errors $\sigma_{u}$ and $\sigma_{v}$, is expressed as [103]

$$
\sigma_{q}^{2}=\left(\frac{\partial q}{\partial u}\right)^{2} \sigma_{u}^{2}+\left(\frac{\partial q}{\partial v}\right)^{2} \sigma_{v}^{2}+2 \frac{\partial q}{\partial u} \frac{\partial q}{\partial v} \sigma_{u v} .
$$

The covariance, $\sigma_{u v}$, is estimated using the Schwartz inequality [103]

$$
\left|\sigma_{u v}\right| \leq \sigma_{u} \sigma_{v}
$$

For the common special case where $u$ and $v$ are independent quantities, the last term in Equation 2.23 is equal to zero [103].

Using this method to determine the uncertainty in the absorbed close, $\dot{\sigma}_{D}$, the error from each event size and in the alpha event size are propagated as independent quantities to get

$$
\sigma_{D}^{2}=k^{2}\left[\sum N(y) y^{2}+\sum \sigma_{y}^{2} N(y)^{2}\right]
$$

To determination the error in dose equivalent values, a simplification of the dose equivalent expression is useful. Substituting Equations 2.18, 2.19, and 2.20 into Equation 2.21, the dose equivalent can be expressed as

$$
H=k\left[0.8 \sum_{y=y_{\min }}^{y_{\max }} y N(y)+0.14 \sum_{y=y_{\min }}^{y_{\max }} y^{2} N(y)\right] \text {. }
$$

The error in the first term is equal to $0.8 \sigma_{D}$. Setting

$$
T=k \sum y^{2} N(y)
$$

then the uncertainty in $T$ is

$$
\sigma_{T}^{2}=4 \sum y^{2} \sigma_{y}^{2} N(y)^{2}+\sum y^{4} N(y) .
$$


The error in the second term of Equation 2.26 is then equal to $0.14 \sigma_{T}$. Since these two terms are derived from the same event size spectrum, they are dependent quantities. The uncertainty in the dose equivalent is then

$$
\sigma_{H}^{2}=k^{2}\left[(0.8)^{2} \sigma_{D}^{2}+(0.14)^{2} \sigma_{T}^{2}+0.224 \sigma_{D} \sigma_{T}\right]
$$

Similarly, the uncertainty in the quality factor is

$$
\sigma_{Q}^{2}=(0.14)^{2}\left(\frac{T}{D}\right)^{2}\left[\left(\frac{\sigma_{D}}{D}\right)^{2}+\left(\frac{\sigma_{T}}{T}\right)^{2}+2 \frac{\sigma_{D} \sigma_{T}}{D T}\right]
$$

Inclusion of the covariant term in the dose-equivalent and quality factor error analysis were found to increase the values of the estimated uncertainties by approximately $2 \%$. For example, inclusion of the covariant term increases the error in $100 \pm 10$ to $100 \pm 10.2$. Since effect on the exror is small, the covariant term was ignored in the error analysis.

\subsubsection{Plotting Techniques for Proportional Counter Data}

Visual separation of the events produced by different charged particles crossing a tissue-equivalent proportional counter is enhanced by operating on the pulse height spectrum. Since the area under the resultant curve is proportional to the absorbed dose, contributions of different charged particles to the absorbed dose can be observed.

Using the calibration procedures outlined in Section 2.5.6, channel numbers are converted to event sizes $\left(\mathrm{keV} \cdot \mu \mathrm{m}^{-1}\right)$. The number of events at a given event size, $N(y)$, is converted to the dose at that event size, $d(y)$, by multiplication of 
the number of events by the dose per event at that event size.

$$
d(y)=k \cdot y \cdot N(y)
$$

This value is then multiplied by the event size. Data are then plotted in the form $y \cdot d(y)$ vs. $\log _{10} y$. The fact that the area under the curve is proportional to the absorbed dose is easily shown:

$$
\int_{0}^{\infty} y \cdot d(y) \mathrm{d}\left(\log _{10} y\right)=\ln 10 \cdot D
$$

To further enhance features on spectra with poor statistics, plotted data are averaged over equal logarithmic intervals. To eliminate $r^{-2}$ fall off, $y \cdot d(y)$ values are multiplied by the distance between the target and detector centers squared.

\subsubsection{Curious Behavior of TEPCs}

The $125.5 \mathrm{~mm}$ diameter proportional counters were especially sensitive to noise. After investigation, it was found that the high humidity present in the detector tubes was the cause of periodic large increases in the noise level. To eliminate the noise, the detectors were thoroughly dried, heated, and sealed with a desiccant in the detector holders. This eliminated noise bursts observed in the $125.5 \mathrm{~mm}$ counters.

Noise persisted at low levels all of the time. The major cause of this problem was ground loops and RF pickup from the accelerator. To cure these problems, detector amplifiers and power supplies were operated from a single point ground in the shielding enclosure. The ground was directly traceable to the data aquisition area where the ADCs and computers resided. This considerably reduced the low level noise. 


\subsection{Beam Monitoring}

In addition to measuring absorbed dose at numerous depths and angles in the shield, the number of protons incident upon the target during each measurement is needed to allow determination of the dose per proton. Furthermore, verification that all protons strike the target and interact minimally with other material in the extracted beam line must be accomplished. This is done using direct beam monitoring devices.

A schematic layout of components in the extracted beam line is shown in Figure 2.9. The distance from the quadrupole exit window to the target location is $570 \mathrm{~cm}$.

\subsubsection{Ionization Chambers}

..n ionization chamber is a device in which ions produced in the filling gas are collected in an electric field and the charge collected recorded. For fast protons, the ionization produced is a result of direct gas ionization from the fast protons [89].

Parallel-plate transmission ionization chambers (TICs) measured the number of protons incident upon the target. Two TIC's are used, one upstream near the quadrupole exit and the other near the target. This allows for verification of beam transport to the target location. The ionization chamber nearest the target measures the number of protons incident upon the target.

The TIC nearest the target location is an air filled device with copper clad glass-epoxy printed circuit board material walls (Figure 2.10). The electrodes 


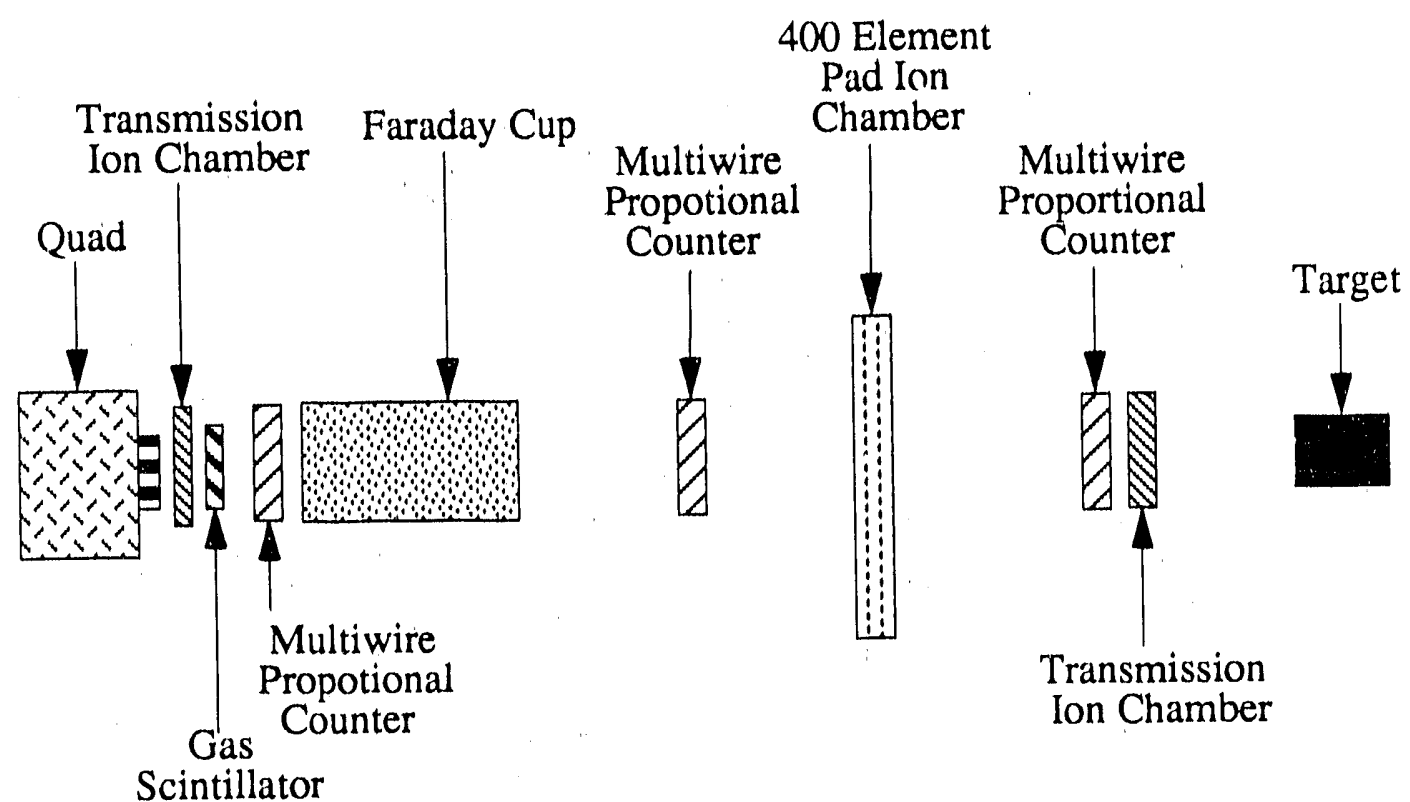

Figure 2.9: A schematic layout of the extracted beam line is illustrated. The transmission ion chamber nearest the target was used as the primary beam monitor. 
are fully guarded by bringing the signal and high voltage lines through the back side of the plates and embedding them in a sandwich between the circuit board material and a sheet of copper clad mylar. The $6 \mathrm{~mm}$ gap collection is maintained by a Lucite spacer. The collector diameter $(12 \mathrm{~cm})$ is larger than the beam diameter so signal from all protons is recorded. The typical operating potential gradient for this device is $135 \mathrm{~V} \cdot \mathrm{mm}^{-1}$.

The upstream $\mathrm{TIC}$ is also an air filled device. To minimize the mass in the beam path, it is constructed of stretched aluminized mylar over a $12.12 \mathrm{~cm}$ Lucite frame that delimits the collection area. To minimize ion recombination, this device has a small gap, $3 \mathrm{~mm}$, and operates at a high potential gradient, $835 \mathrm{~V} \cdot \mathrm{mm}^{-1}$. Guarding is accomplished through a ground plane inserted between the collection and high voltage planes.

Ionic recombination as a function of beam intensity is determined by taking voltage saturation measurements and comparing the output with a fully saturated chamber. Voltage saturation data are used to determine the fraction of ions recombining as a function of potential [20]. This method consists of recording the fraction of the charge collected at a given potential, changing the potential, and noting the change in the charge collected. For general recombination in a continuous beam, a plot of $\mathrm{Q}^{-1}$ vs, $\mathrm{V}^{-2}$ yields a linear plot, with the intercept equal to $Q_{s a t}^{-1}$. Figure 2.11 shows a plot of the recombination as a function of beam intensity for the target TIC along with values predicted using Boag's theory of ionic recombination [32]. These data were obtained using a modified beam line in which the beam was transported in vacuum to the target focation with 


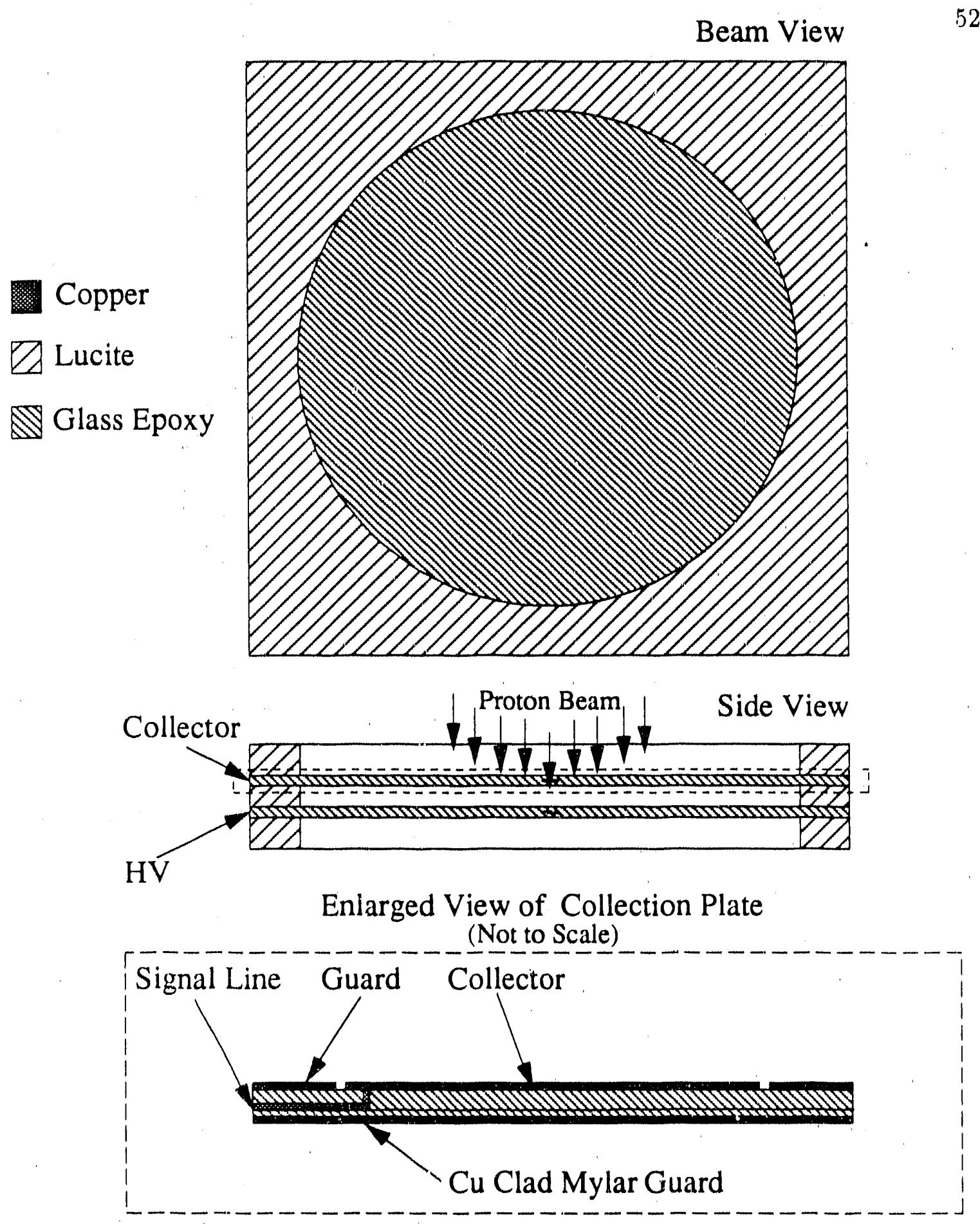

Figure 2.10: Schematic diagram of the transmission ionization chamber located nearest the target location. The walls are constructed out of printerl circuit board material and are separated by a $6 \mathrm{~mm}$ Lucite spacer. The enlarged section shows how signals were brought out in a fully guarded manner by enchosing the signal line between ground planes. 
all upstream monitoring devices removed. The beam spot diameter at the target location was less than $1 \mathrm{~cm}$.

Differences between Boag's theory and measured values are attributed to difficulties in determination of the absolute beam size for normalizing the intensity values. The relative intensities are believed to be correct. The points that deviate significantly from the slope of the theoretical curve are caused by the extracted beam intensity time structure. During those measurements, the beam was not uniformly extracted over the $0.8 \mathrm{sec}$ duration. Rather large variations existed, with the majority of the beam being extracted in a few tens of milliseconds. Fortunately, the upstream ionization chamber was also in place at the downstream location during these measurements. The upstream TIC showed less than one percent ion recombinations at all bean intensities due to its small gap and high electric field. This device was used to determine the number of protons incident upon the target for these runs. In measurements with the nomal beam line configuration, (Figure 2.9), both the downstream and upstream TIC's exhibited minimal ion recombination due to the larger beam sizes.

An implication of rate dependent recombination is that ion chambers used to monitor beam intensity during patient treatments must not show this rate effect, else patients will be treated to improper levels. For patient treatments, ion chambers similar in design to the upstream ion chamber (small gap, high field) should be used to defer this effect to much higher beam intensities. 


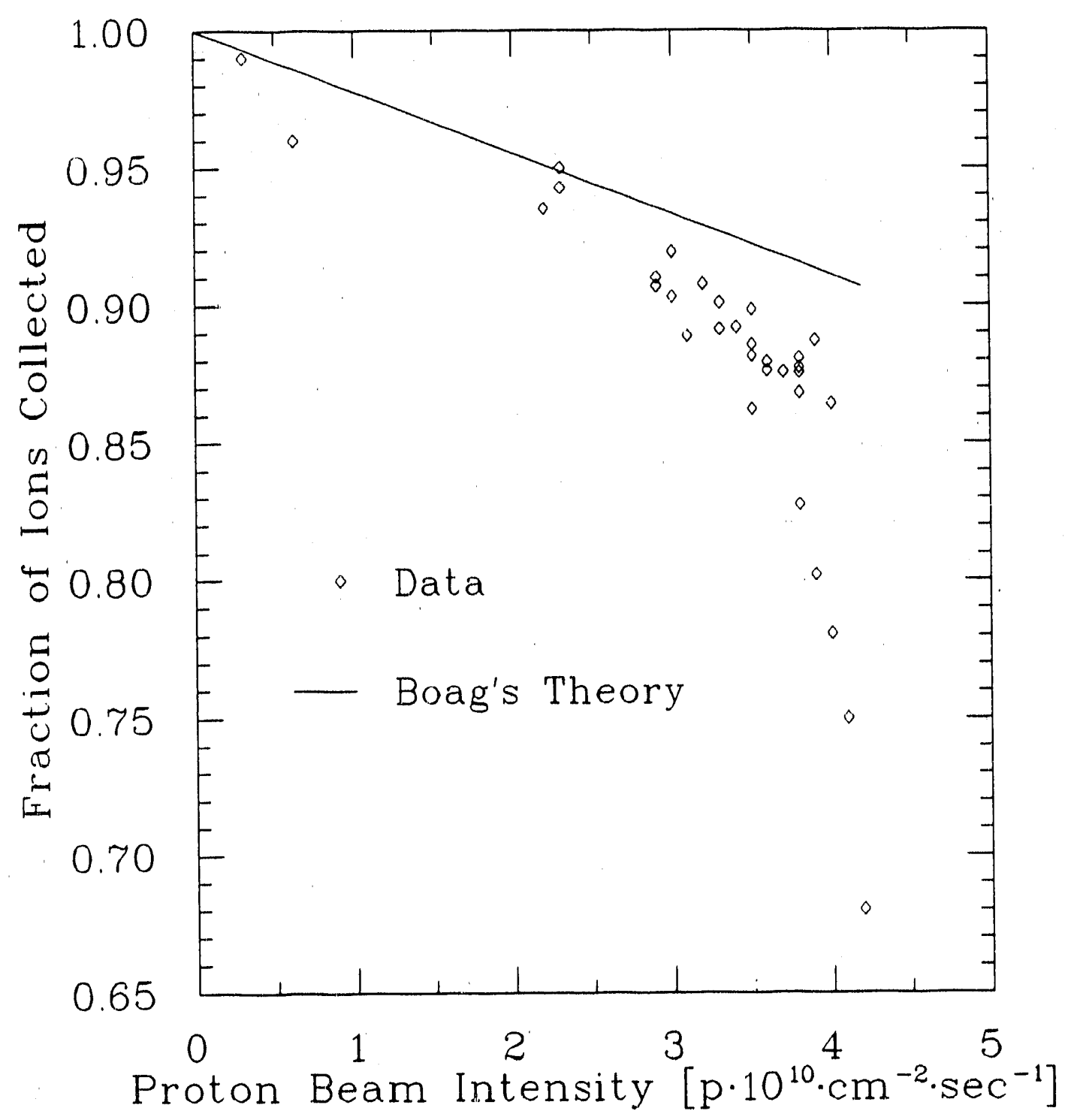

Figure 2.11: Graph of the fraction of ions collected 4s. the proton beam intensity. Large recombination values are observed due to the low operating potential of this device. 


\subsubsection{Ionization Chamber Calibrations}

Monitor ionization chamber calibration allows conversion of the charge collected, $Q$, to the number of protons, $N$, crossing the counter. The response of an ionization chamber in a heavy charged particle beam is computed from

$$
\frac{Q}{N}=\frac{d E}{\rho d x} \rho \Delta x \frac{e}{\bar{W}}
$$

where $\frac{d E}{p d x}$ is the stopping power of the gas in the ionization chamber evaluated at the energy of the proton that crosses the chamber, $\rho$ is the density of the gas in the chamber, $\Delta x$ is the plate separation between which ions are produced, and $\frac{W}{e}$ is the mean energy required to produce an ion pair in the gas [89]. The anticipated response for the downstream TIC is listed in Table 2.10.

The actual response of the ionization chamber is measured through calibration with respect to a Faraday cup. The Faraday cup, c'iagramed in Figure 2.12, consists of a re-entrant stainless steel absorber cup $102 \mathrm{~mm}$ deep which is held in vacuum. As the beam enters the cup, a suppressor ring held at $-700 \mathrm{~V}$ serves to forbid knock-out electrons from leaving the cup. The number of particles $\mathrm{N}$ stopped in the Faraday cup is

$$
N=\frac{Q_{F}}{z e}
$$

where $Q_{F}$ (coulombs) is the charge collected, $z$ is the charge per incident particle ( 1 for protons) and $e$ is the electronic charge in coulombs [89].

In calibration with respect to the Faraday cup, the beam first passes through the ion chamber in which the charge $Q_{1}$ is collected, then strikes the Faraday cup from which the charge $Q_{F}$ is collected from the stopping protons. Charge $Q_{F}$ is 

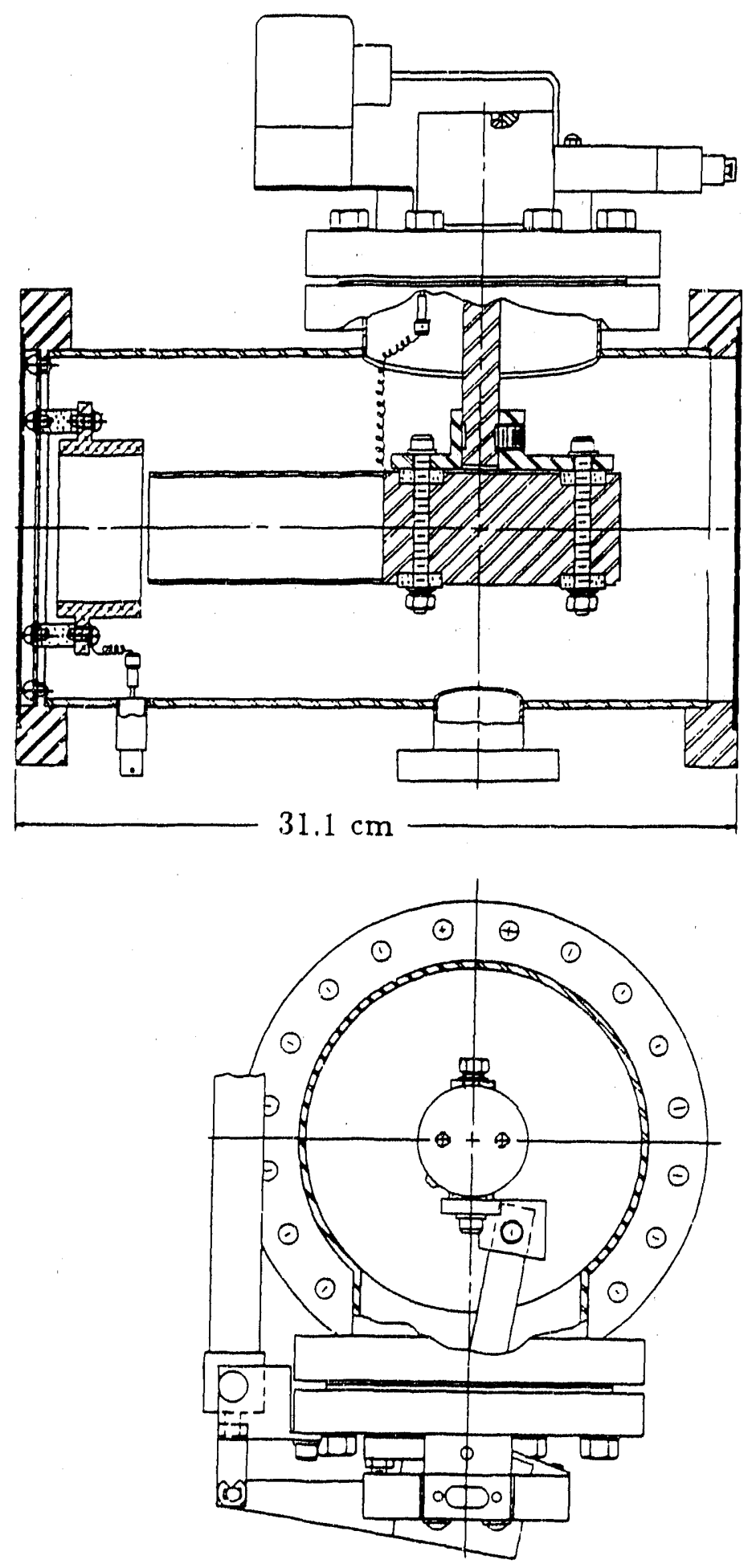

Figure 2.12: Cross-sectional view of Faraday cup. The re-entrant absorber hlurk is constructed out stainless steel (Manufactured by National Electrostatics (orp., Middletion, WI). 


\begin{tabular}{||l|c|c||} 
Location & $\begin{array}{c}\text { Beam Intensity } \\
\left(\mathrm{p} \cdot \mathrm{cm}^{-2} \cdot \mathrm{sec}^{-1}\right)\end{array}$ & $\begin{array}{c}\text { Calibration Factor } \\
\left(\mathrm{p} \cdot \mathrm{fC}^{-1}\right)\end{array}$ \\
\hline Calculated & - & 86.25 \\
Fermilab & $4.00 \cdot 10^{9}$ & 85.45 \\
$\mathrm{HCL}$ & $3.80 \cdot 10^{9}$ & 85.72 \\
\hline
\end{tabular}

Table 2.10: Calibration factors for clownstream transmission ion chambers. The value determined at the Harvard Cyclotron Laboratory (HCL) has been normalized to $230 \mathrm{MeV}$ and to the temperature and pressure conditions present during the Fermilab measurement.

converted to the number of protons stopping in the cup, $N$, using Equation 2.34. The calibration factor, $\frac{Q_{1}}{N}$, is then determined.

Ion chamber calibrations with respect to a Faraday cup were performed both at Fermilab and the Harvard Cyclotron Laboratory (HCLL). Values from HCIL are corrected for beam energy using stopping power ratios, as well as for air density differences using measured air temperatures, pressures and relative humidities. Results of these calibrations are also listed in Table 2.10 for typical beam intensities. Since the ionization chambers show increased recombination with increased proton intensity, values are corrected to a fully saturated chamber $1100 \%$ charge collection).

\subsubsection{Multi-Wire Proportional Counters}

On-line visual verification of beam size and positioning is available using multiwire proportional counters (MWPC's). These devices, shown in Figure 2.13, are also referred to as multi-wire ionization chambers (MWIC's) and scanning wire ionization chambers (SWICs). Since these devices operate in proportional 
counter mode, the term MWPC is preferred. These devices are used to ensure the beam will strike the target and that the beam size is smaller than the target diameter.

The multi-wire proportional counters consist of two planes of wires perpendicular to the beam direction rotated $90^{\circ}$ with respect to each other. Wires are separated by a high voltage mesh $(\approx-1000 \mathrm{~V})$. An $80^{\prime}$ - $\mathrm{Ar} 20 \%-\mathrm{CO}_{2}$ gas mixture flows through the MWPCs. Operation is via gas ionization produced by the crossing proton beam, collection of the ions produced by the electric field, and charge multiplication of the ions in the high field region surrounding the small diameter wires. Wires are spaced at $2 \mathrm{~mm}$ intervals. The charge from each wire is read out using a scanning amplifier system which also processes the data for video presentation [55]. Resultant video cross-sectional beam profiles are updated for each beam pulse. A plot of a typical beam profile is shown in Figure 2.14 .

\subsubsection{Polaroid Film}

In addition to using MWPC's to monitor beam positioning, polaroid film is used for position and size verification. Prior to each run, polaroid films are placed on the target, on the quadrupole exit window, and across the apertures of the transmission ion chambers and MWPCs. A few beam spills are allowed to travel through the beam line, then the films are removed and developed. Any adjustments necessary to eliminate beam scrapping or miss-positioning are made, and a new set of films inserted for re-verification. This procedure is repeated until 

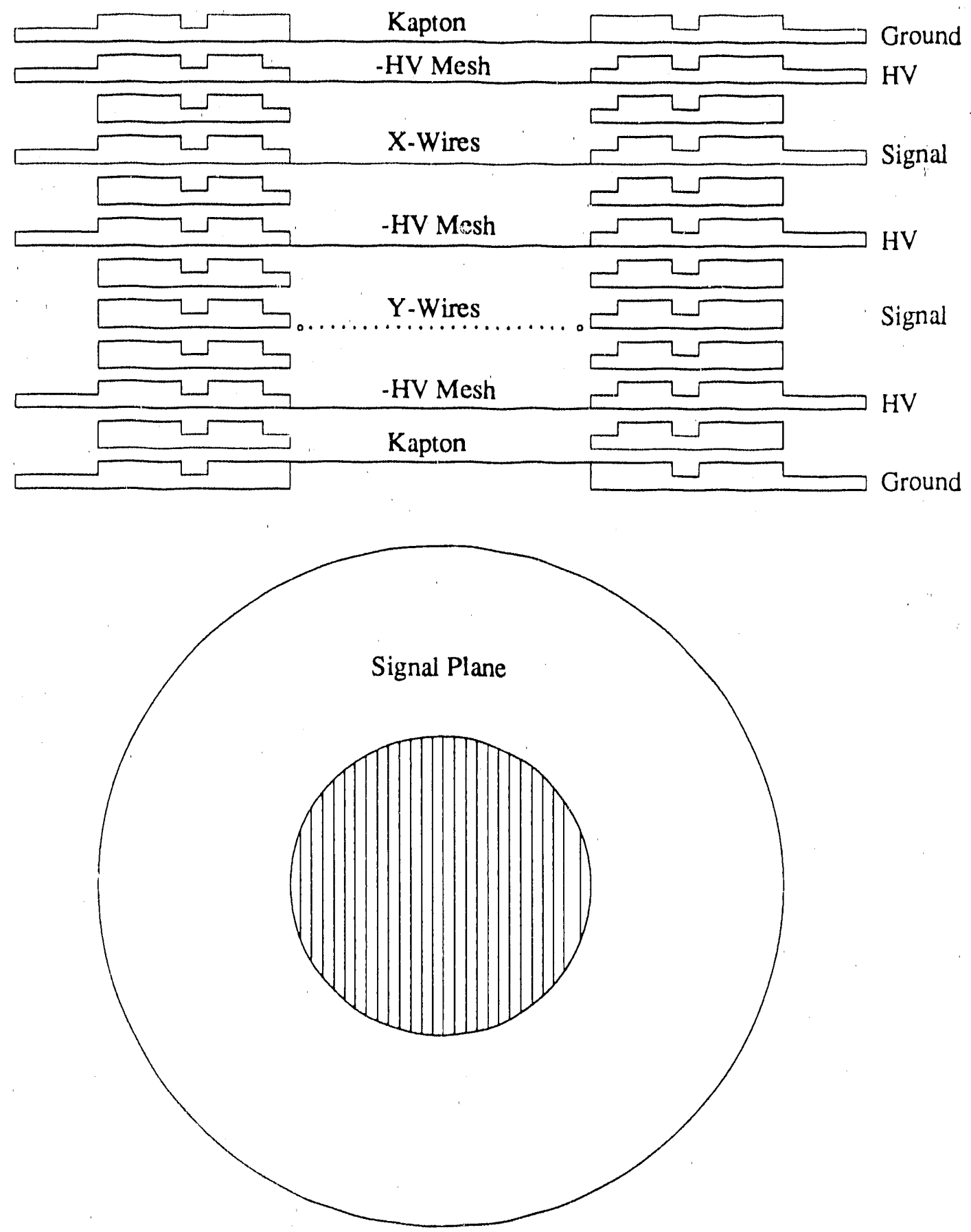

Figure 2.13: Schematic view of multi-wire proportional counters used to measure beam positioning. Signals recorded on the X and Y signal planes are processed for video presentation. 

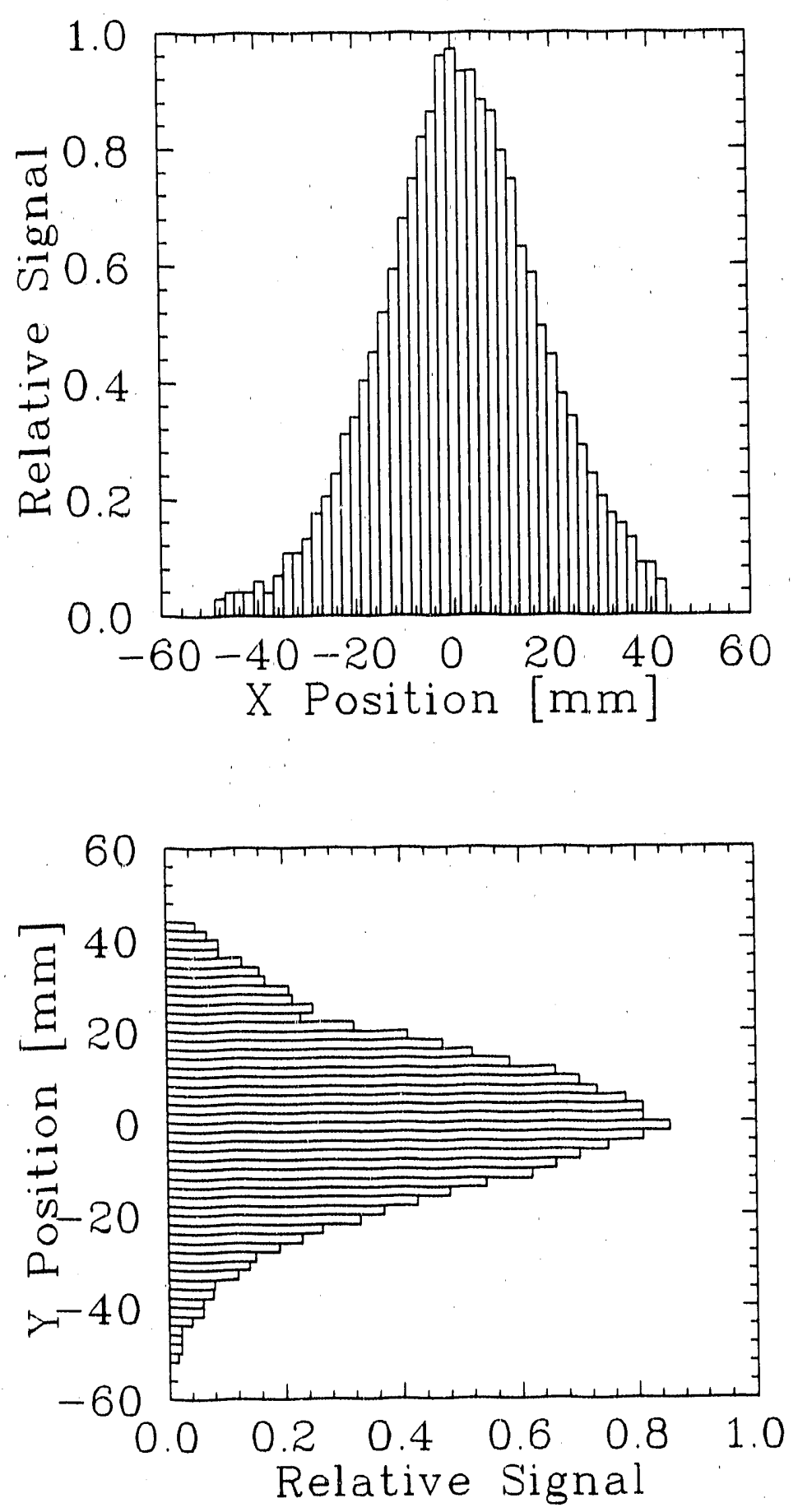

Figure 2.14: Typical beam profiles in the $\mathrm{X}$ and $\mathrm{Y}$ directions as recorded on a multi-wire proportional counter. Wire spacings are $2 \mathrm{~mm}$. 
the beam is properly positioned. 


\section{Chapter 3}

\section{Experimental Results}

In this section, TEPC event size spectra produced by the radiation enviromment in the shield are presented. Spectra are evaluated to determine absorbed dose, quality factors, and dose equivalent values as functions of depth and angle in the shield. Resultant attenuation data at each angle for each target material are presented.

\subsection{Event Size Spectra}

Event size spectra were obtained at several penetration depths at each angle using the low pressure tissue-equivalent proportional counters. Spectra are normalized to the number of protons incident upon the target and transformed into dose plots using methods outlined in Section 2.5.8. To eliminate $\mathrm{r}^{-2}$ falloff, $\mathrm{y} \cdot \mathrm{d}(\mathrm{y})$ values are multiplied by the distance from the detector center to the target squared.

\subsubsection{Qualitintive Analysis of Event Spectra}

Neutron interactions in material surrounding a TEPC create charged particles that produce the observed events. Due to the energy dependence of neutron 
produced events, event size spectra can be used to decipher features of the radiation environment producing the events. This section describes event size spectra features observed at each angle, depth, and target in a qualitative fashion.

The smallest event observable in a TEPC is from the formation of a single ion pair. Given that typical energies required to produce an ion pair are of the order: of $30 \mathrm{eV}$ and counter diameters simulate $2 \mu \mathrm{m}$ of tissue, the smallest event size is $0.015 \mathrm{keV} \cdot \mu \mathrm{m}^{-1}$. Due to total system noise, these single ion pair events are not observable. The smallest observable event size above noise is $0.90 \mathrm{keV} \cdot \mu \mathrm{m}^{-1}$,

Gamma-ray interactions produce events immediately above the electronic lower level discriminator. Figure 3.1 shows a plot of the event size spectrum obtained using a ${ }^{226} \mathrm{Ra}$ gamma-ray source. The principal gamma-rays above $0.5 . \mathrm{MeV}$ emitted by ${ }^{228} \mathrm{Ra}$ have energies of $0.607,1.120$, and $1.761 \mathrm{MeV}$. Since these gammas interact primarily by Compton interactions [63], the secondary electron energy spectrum produced by gamma-rays in a TEPC is nearly independent of gamma ray energy. In the shielding materials, gamma-rays are produced by radiative capture and inelastic interactions and have energies of a few $\mathrm{MeV}[51,83,105]$. Therefore, the event spectrum produced by ${ }^{226} \mathrm{Ra}$ gamma-rays is similar to that produced by gamma-rays in the shield.

For high energy neutrons, the principal component of the event size spectrum is protons from $(n, p)$ interactions. The University of Wisconsin Gas Target Neutron Source (GTNS), described in detail by DeLuca et al. (41), produced the 14.8 MeV neutrons from which the spectrum in Figure 3.2 is obtained. While a small fraction of gamma events at low event sizes is observed, the spectrum is 


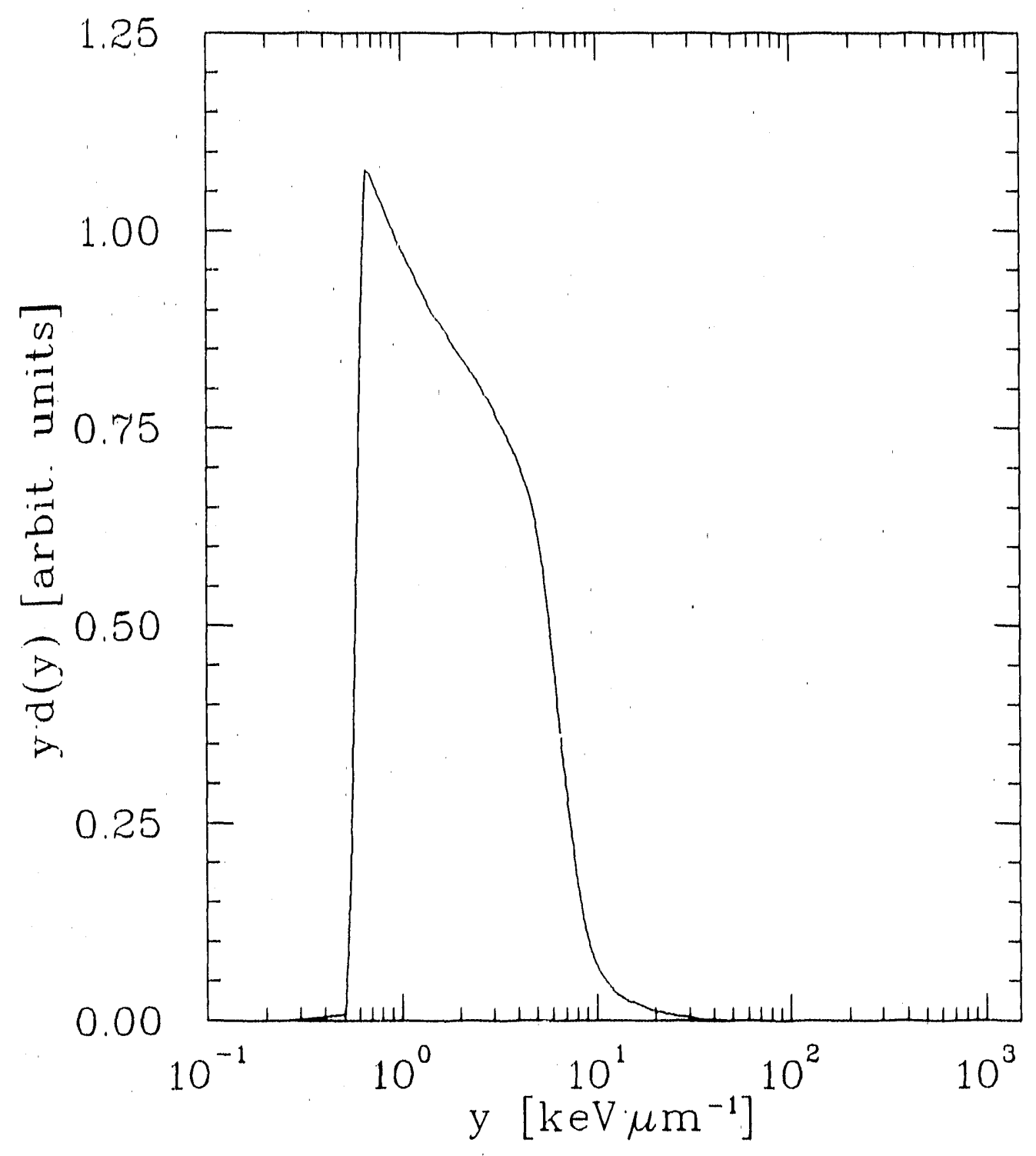

Figure 3.1: Event size spectrum induced by gamma-ray emitted from a ${ }^{226} \mathrm{Ra}$ source, average gamma-ray energy $\approx 1.25 \mathrm{MeV}$, maximum energy $2.2 \mathrm{MeV}$. 
dominated by larger events produced by heavy charged particles. Stopping power increases with decreasing energy, hence, larger event sizes are caused by lower energy heavy charged particles. Events from a few $\mathrm{keV} \cdot \mu \mathrm{m}^{-1}$ to $\approx 100 \mathrm{keV} \cdot \mu \mathrm{m}^{-1}$ are caused by protons. The sharp fall off at $\approx 100 \mathrm{keV} \cdot \mu_{\mathrm{m}}^{-1}$ results from low energy protons which deposit the maximum energy possible for proton events. This feature is called the proton edge, proton drop point or Bragg edge. Events above the proton edge are caused by other heavy charged particles crossing the counter such as alpha particles and carbon recoils.

The peak in the proton events at $\approx 1,0 \mathrm{keV} \cdot \mu \mathrm{m}^{-1}$ is produced by the largest component of the neutron spectıum. A higher average neutron energy will produce, on an average, higher energy protons. For higher average energy neutrons, this proton peak occurs at smaller event sizes. For low energy neutrons, this peak is displaced to larger event sizes, with very low energy neutrons producing protons near the proton edge.

Event size spectra plots obtained at $0^{\circ}$ for the Fe target are shown in Figures 3.3. At $21 \mathrm{~cm}$ penetration, the neutron spectrum is dominated by fast proton events. Comparing this spectrum to the $14.8 \mathrm{MeV}$ neutron spectrum, (Figure 3.2), it is observed that the location of the fast proton peak decreases from $8 \mathrm{keV} \cdot \mu \mathrm{m}^{-1}$ to $5 \mathrm{keV} \cdot \mu \mathrm{m}^{-1}$, thus, the average neutron energy is higher. At $84 \mathrm{~cm}$ and $148 \mathrm{~cm}$ penetrations, intensity decreases considerably, yet the spectral shape is similar, with a slight hardening observed by the decrease in the location of the fast proton peak.

Event spectra at $90^{\circ}$ are quite different as shown in Figure 3.4. At $20 \mathrm{~cm}$ 


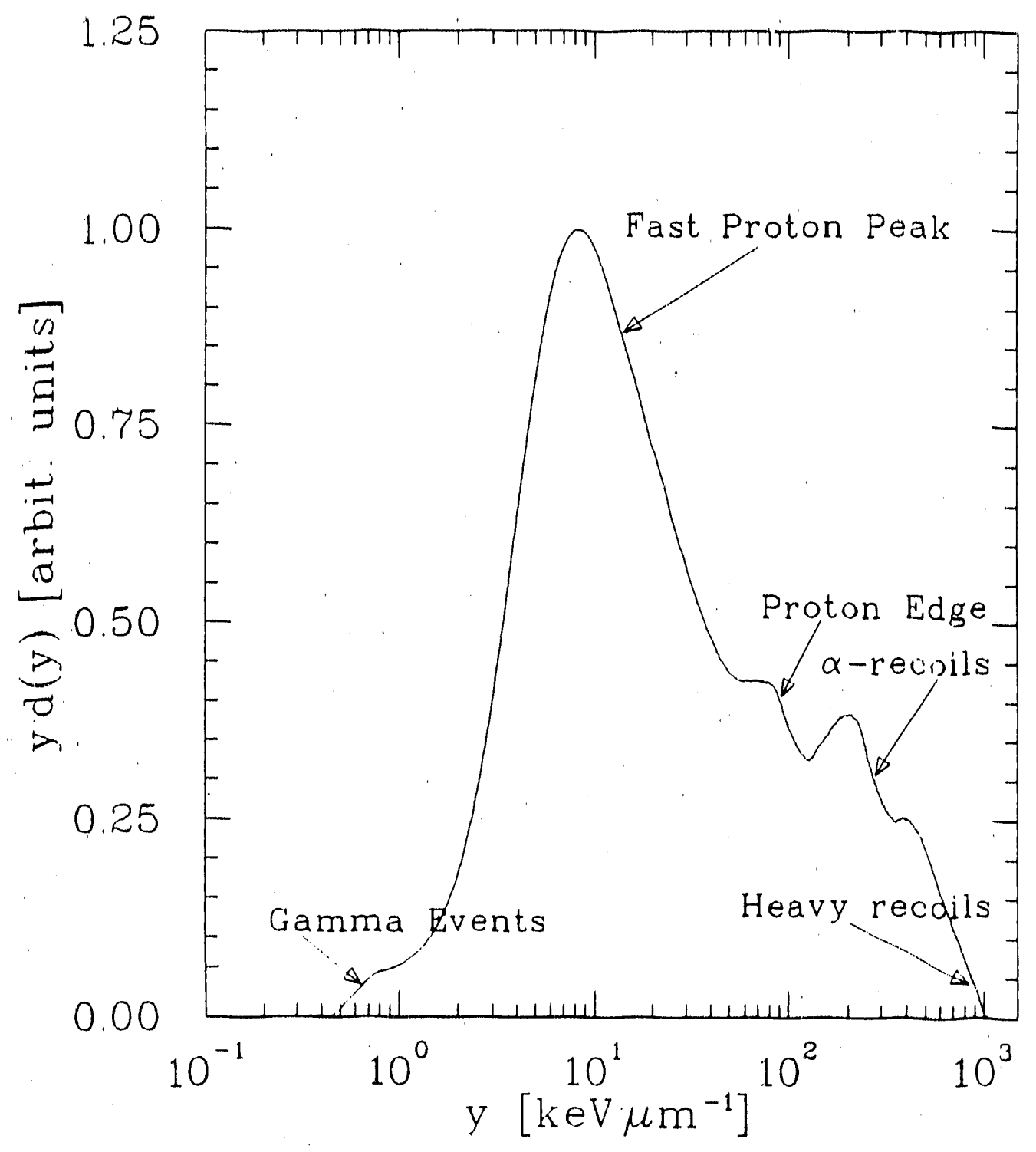

Figure 3.2: $14.8 \mathrm{MeV}$ monoenergetic neutron induced event size spectrum using GTNS. Important features are noted and discussed in text. 


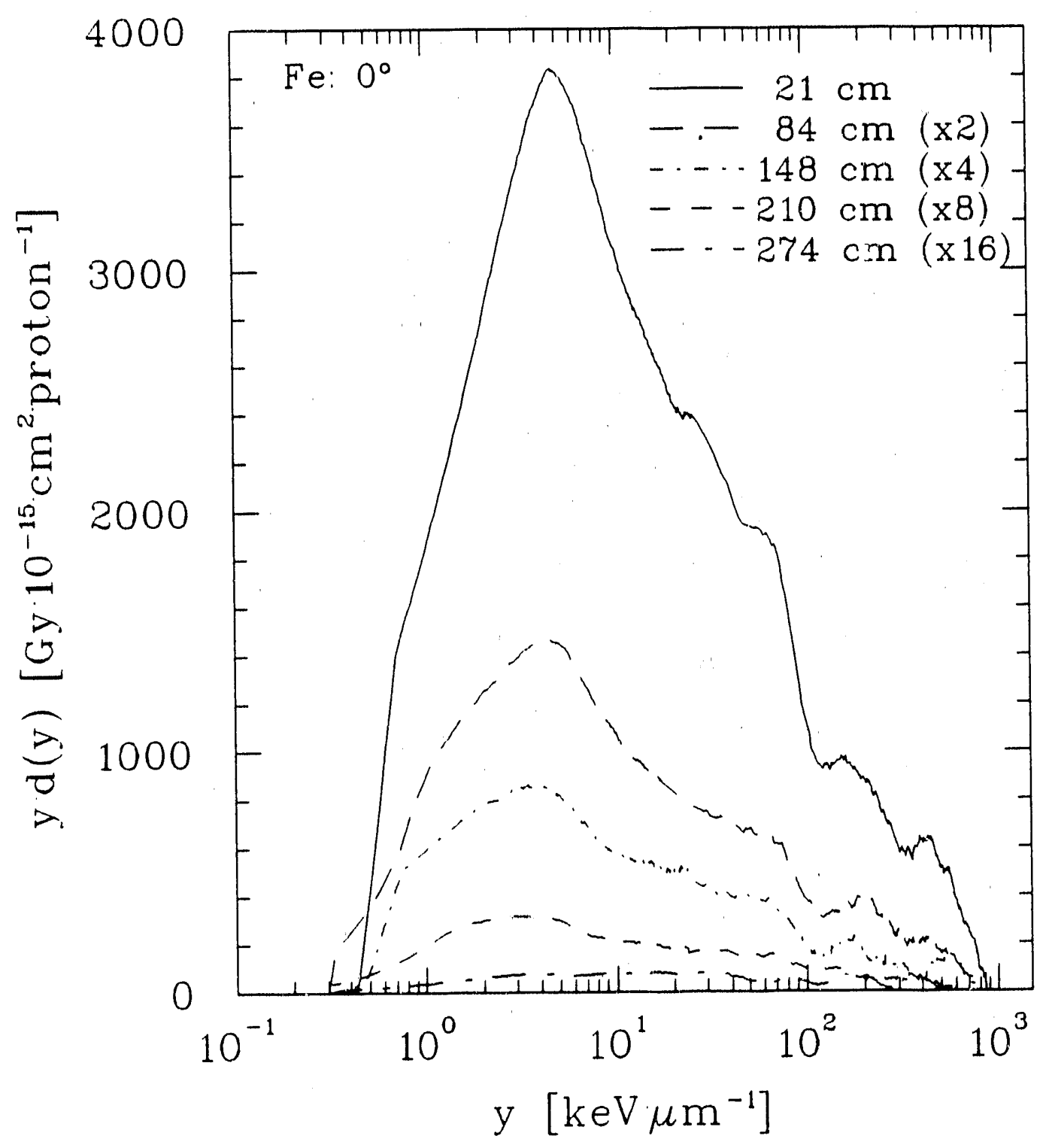

Figure 3.3: Event spectra observed at $0^{\circ}$ for each depth into the shield with $230 \mathrm{MeV}$ protons incident upon an Fe target. 
penetration, the fast proton peak is still visible, yet the spectrum is dominated by events near the proton edge produced by low energy neutrons. The spoctrum is considerably hardened at $61 \mathrm{~cm}$ and $105 \mathrm{~cm}$ where only a residual hard component persists.

The angular dependence of neutron production is shown in greater detail in Figure 3.5 which shows a plot of the spectra obtained $20 \mathrm{~cm}$ into the concrete shield at each angle. Here, the sharp falloff of the neutron production, as well as the softening of the neutron spectrum with increasing angle is apparent.

Figures 3.6-3.7 show plots of event size spectra observed at $22^{\circ}$ and $45^{\circ}$ for Fe target bombardment. The soft neutron component increases in relative importance with increasing angle. This is not caused by an increase in low energy neutron production with angle, but rather a decrease in the high energy neutron components. By the second penetration, low energy components are largely attenuated, and an equilibrium spectrum propagates and attenuates through the shield. At deep penetrations, average event size increases with angle. Therefore, it can be deduced that the average neutron energy decreases with increasing angle.

Target differe ces are most evident $20 \mathrm{~cm}$ into the shield at each angle, in particular at $90^{\circ}$ (Figure 3.8 ). The $\mathrm{Pb}$ target data exhibit a significantly larger low energy neutron component than the $\mathrm{Fe}$ and $\mathrm{Al}$ data. Further into the shield, target variations are minimal in both magnitude and shape (Figure 3.9) Therefore, the additional neutron production in the $\mathrm{Pb}$ target can be attributed almost entirely to low energy neutron production. 


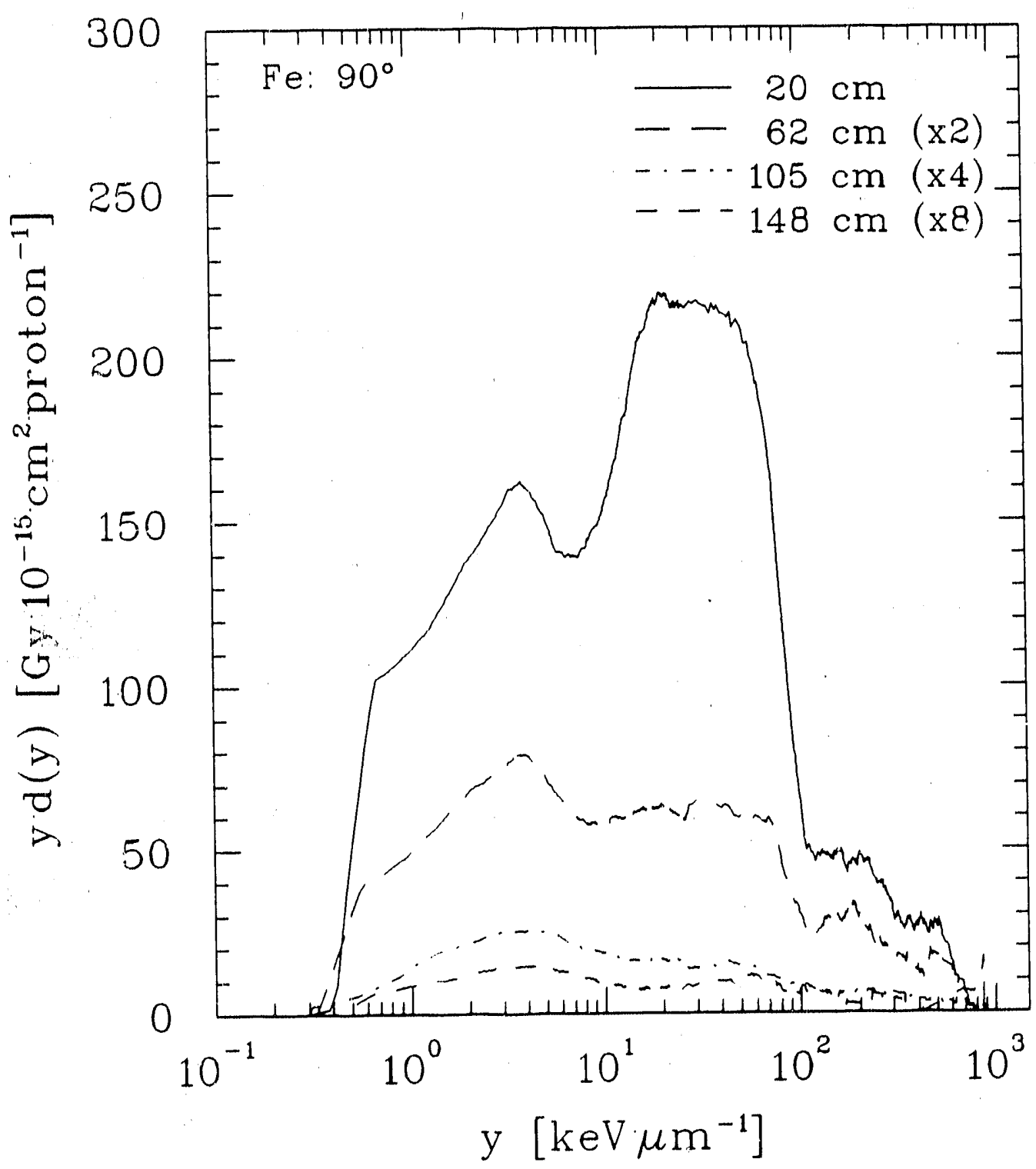

Digure 3.4: Event spectra observed at $90^{\circ}$ for each depth into the shield with $230 \mathrm{MeV}$ protons incident upon ar Fe target. 


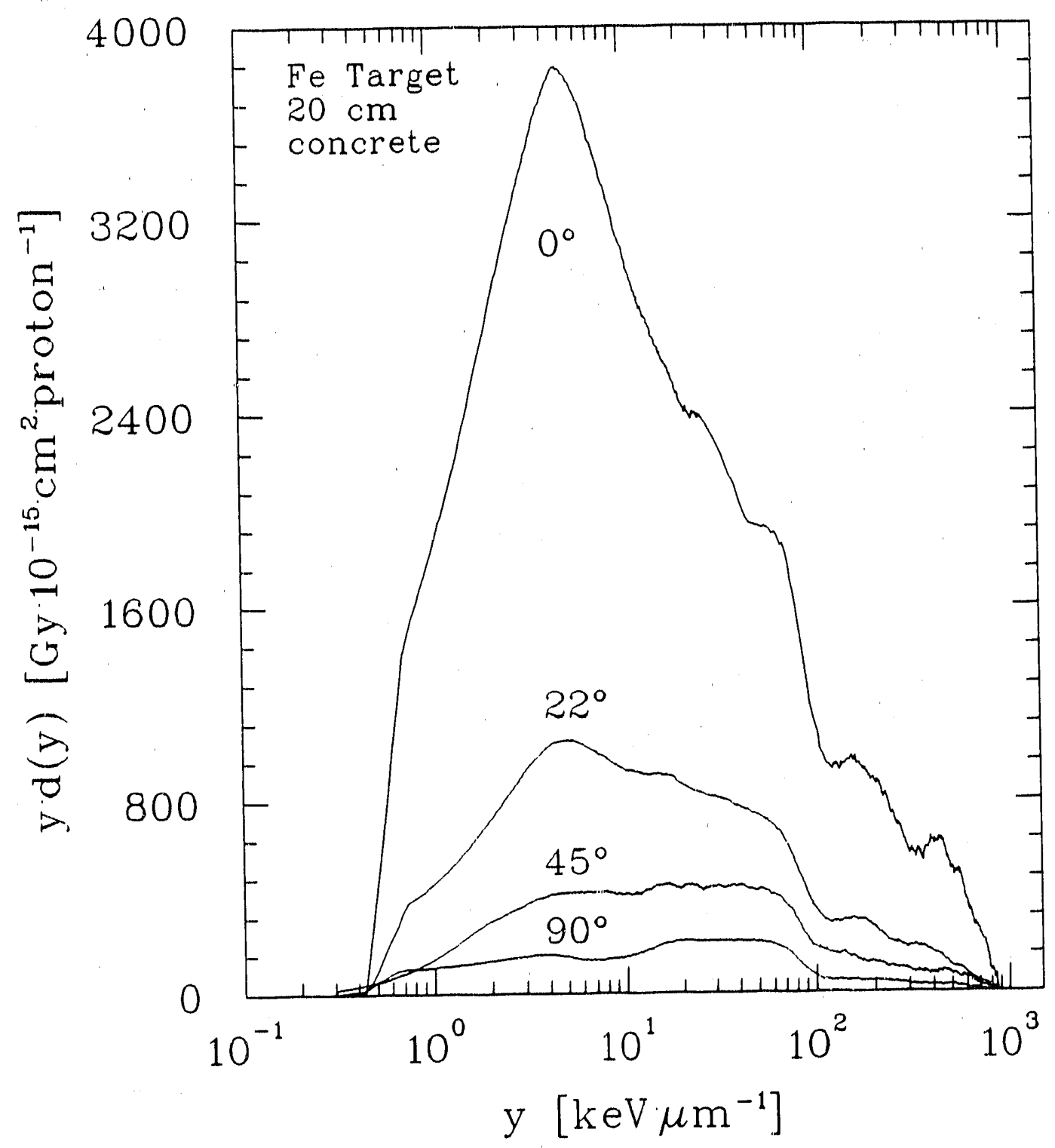

Figure 3.5: Event spectra observed at each angle, $20 \mathrm{~cm}$ into the concrete shield for $230 \mathrm{MeV}$ proton bombardment of an Fe target. 


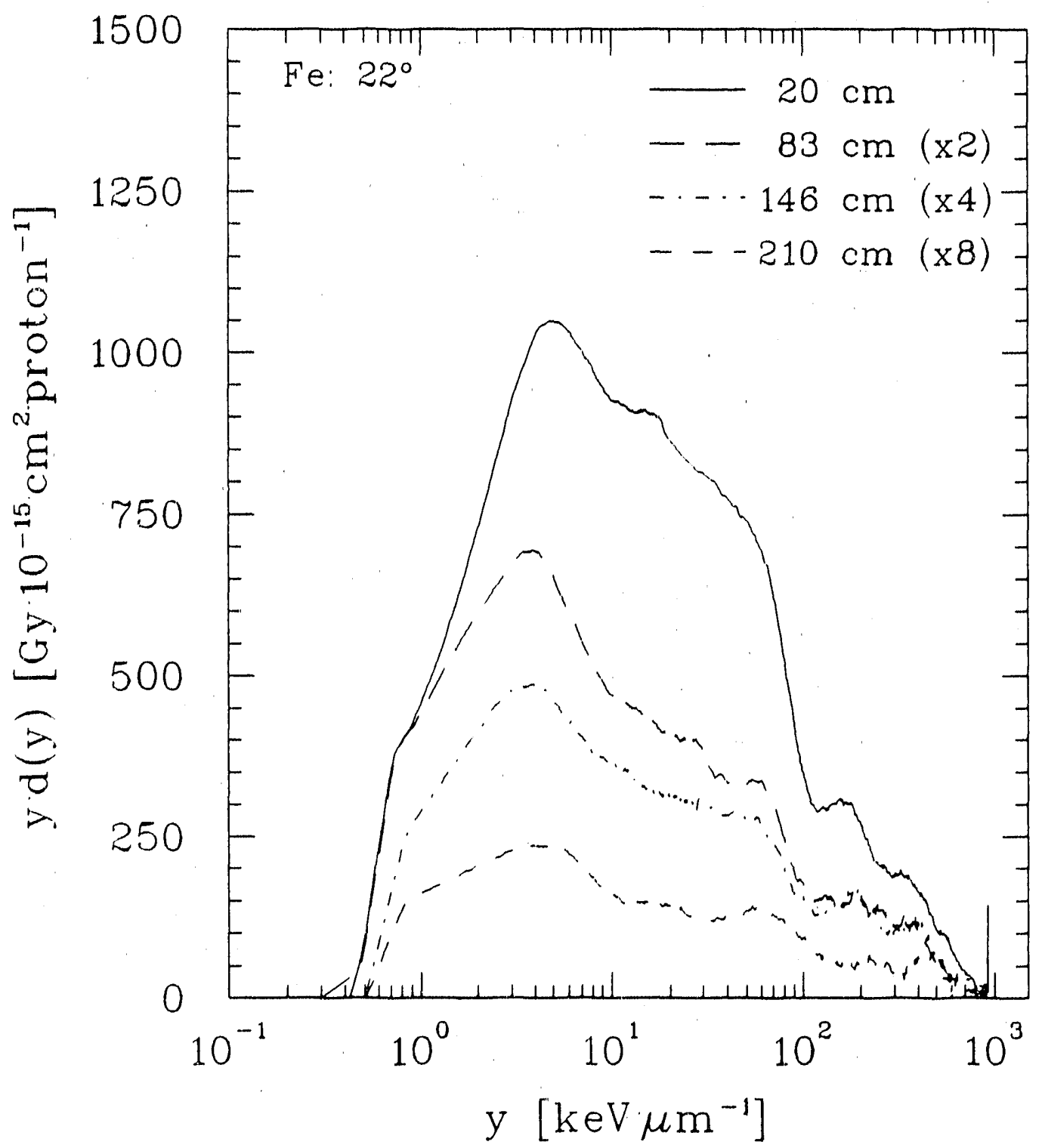

Figure 3.6: Event spectra observed at $22^{\circ}$ for each depth into the shield with $230 \mathrm{MeV}$ protons incident upon an Fe target. 


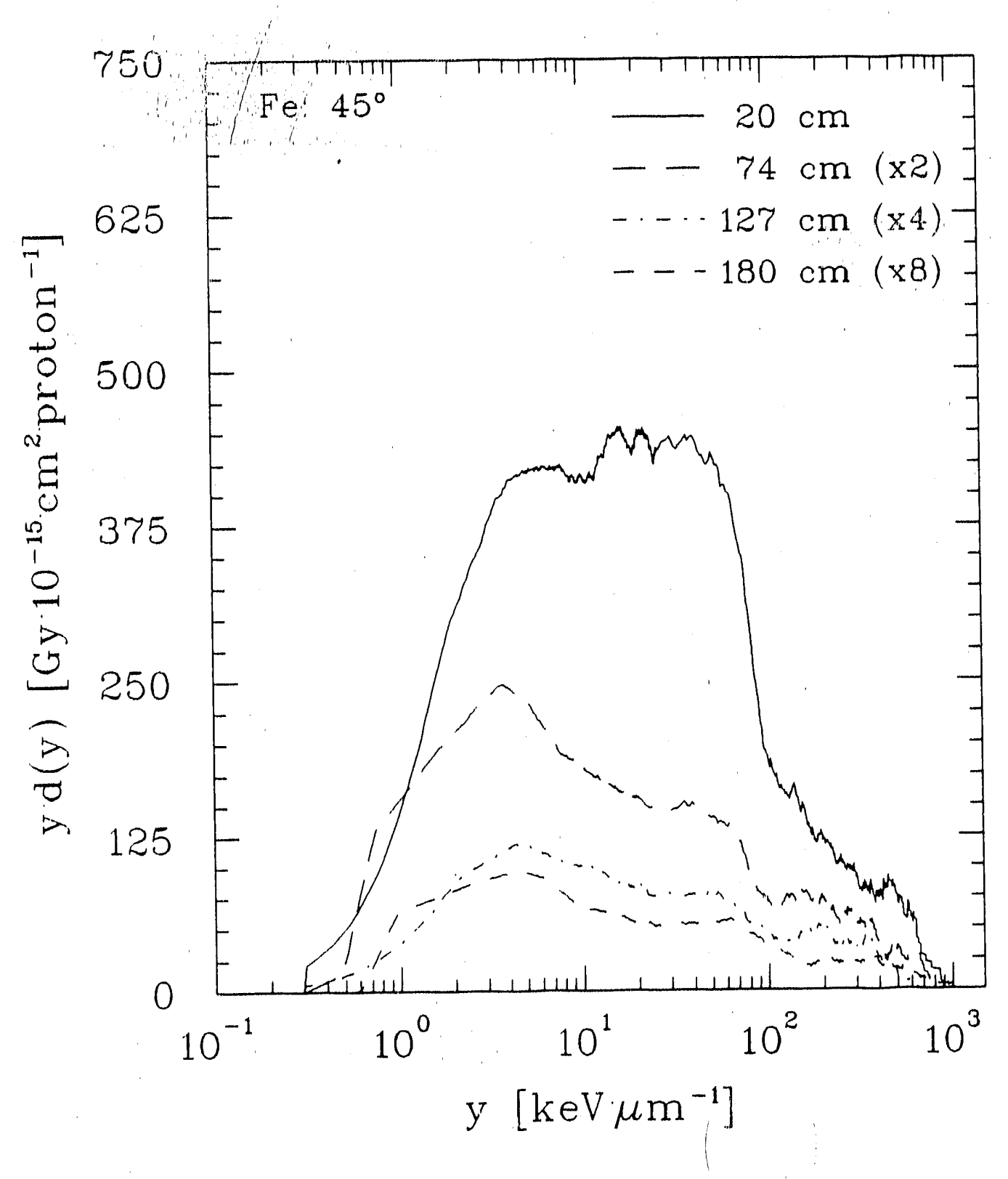

Figure 3.7: Event spectra observed at $45^{\circ}$ for each depth into the shield with $230 \mathrm{MeV}$ protons incident upon Fe an target. 


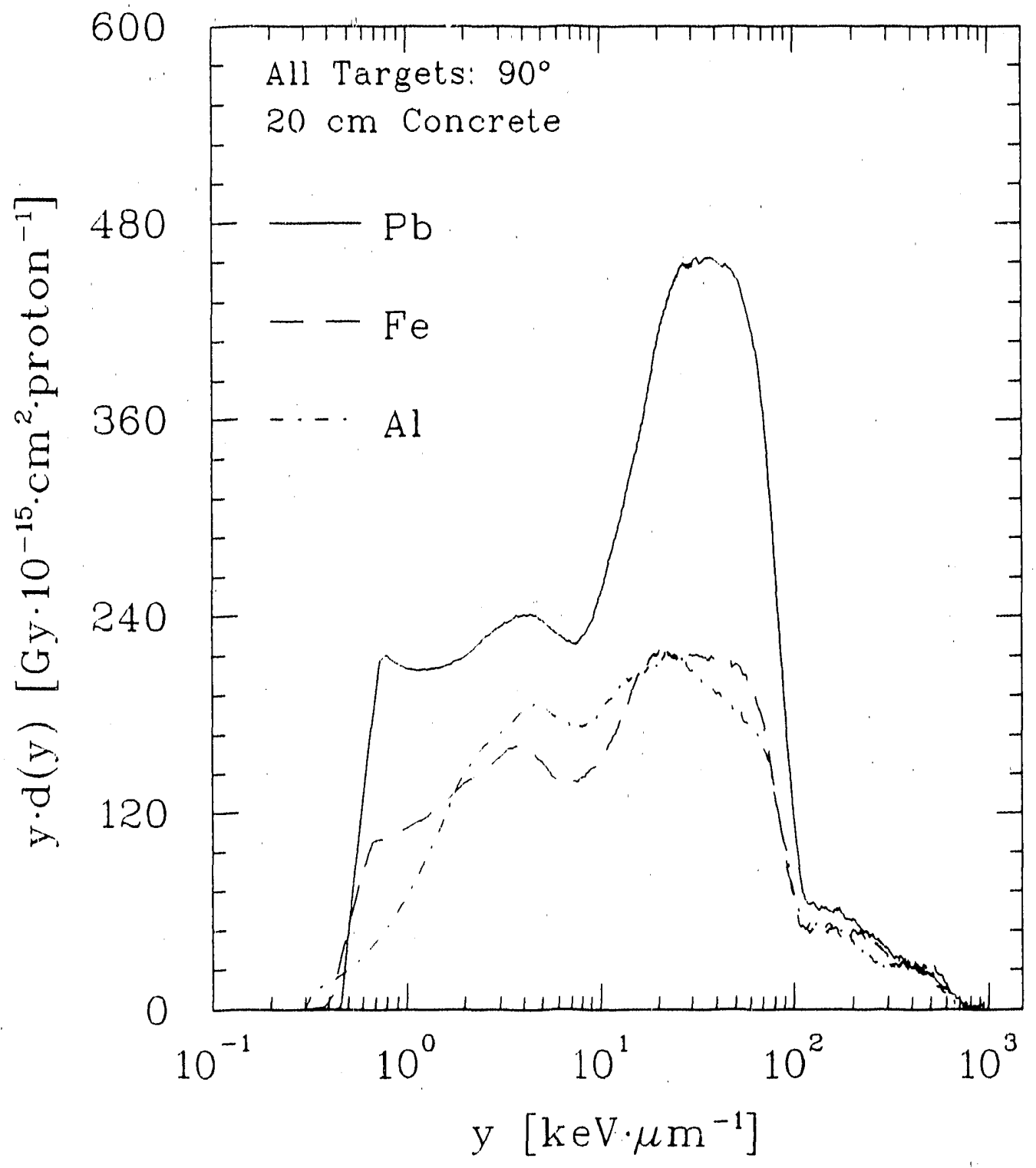

Figure 3.8: Event spectra observed $20 \mathrm{~cm}$ into the concrete shield at $90^{\circ}$ with respect to each target for $230 \mathrm{MeV}$ proton bombardment. 


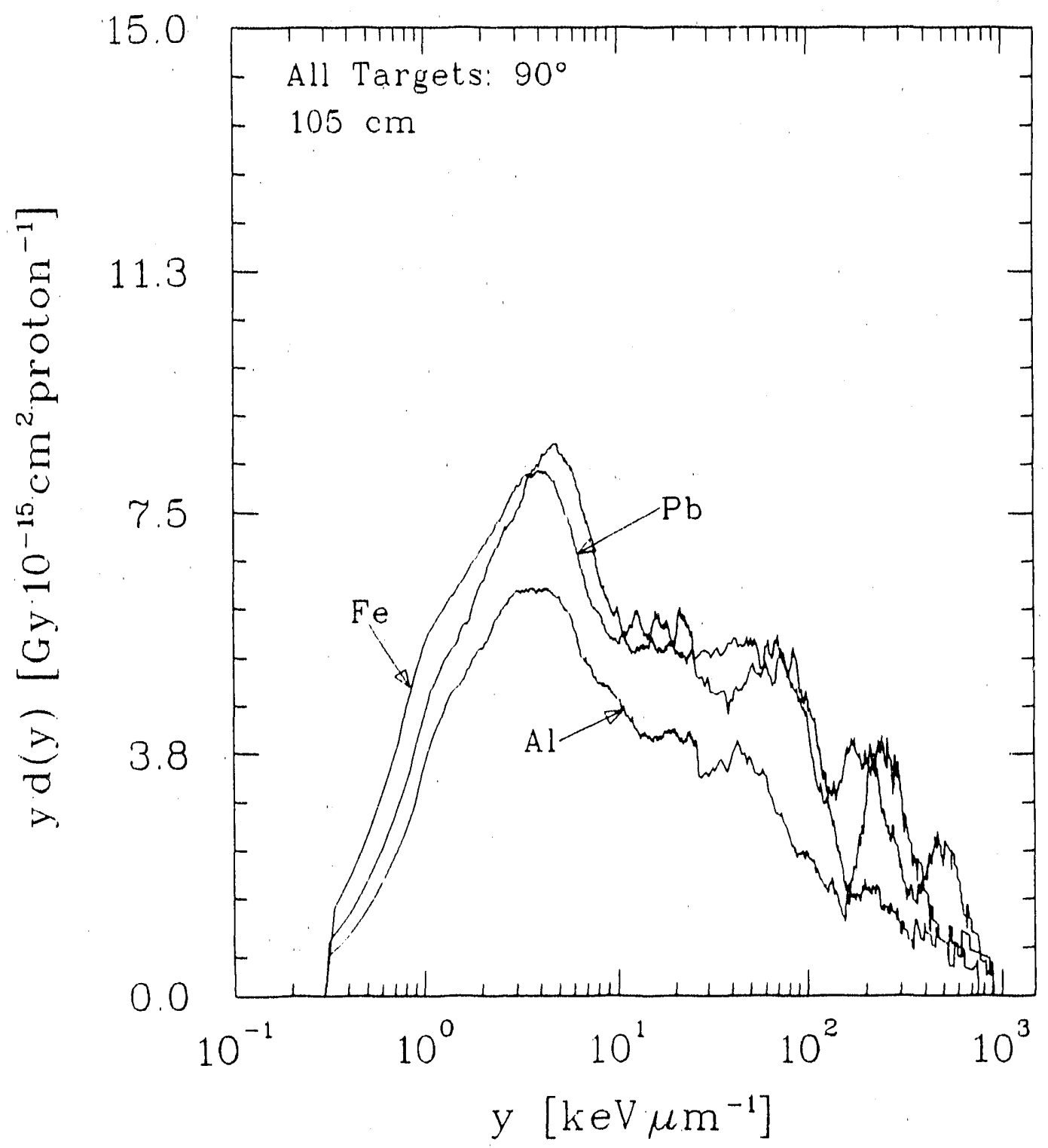

Figure 3.9: Event spectra observed $105 \mathrm{~cm}$ into the concrete shield at $90^{\circ}$ with respect to each target for $230 \mathrm{MeV}$ proton tombardment. 
Other event data at depth in the shield for $\mathrm{Al}$ and $\mathrm{Pb}$ targets are similar to the Fe data. Figures 3.10-3.17 show complete sets of event size spectral plots for the $\mathrm{Pb}$ and $\mathrm{Al}$ targets at all four angles. $\mathrm{Pb}$ data in the first depth at $0^{\circ}$ were affected greatly by gain shifts in the amplifier system due to the extreme data rates. Al and $\mathrm{Fe}$ data were obtained with much lower beam intensities, hence lower data rates. The $\mathrm{Pb}$ spectral shape in the first penetration at $0^{\circ}$ is probably similar to the Fe and Al target data. Spectral shapes for large penetrations are observed to be independent of target material.

At larger angles, low energy neutrons are noticeable at the shallow penetrations, then attenuated leaving a residual hard component at large penetration values. An interesting feature of the shallow penetration data at large angles is the peak at small event sizes $\left(\approx 10 \mathrm{keV} \cdot \mu \mathrm{m}^{-1}\right)$ corresponding to the high energy component of the neutron spectrum. When a multiple of the high energy neutron spectrum is subtracted from this spectrum, for example a deep penetration at $0^{\circ}$, the residual spectrum (Figure 3.18) shows many interesting features such as the remaining gamma events and events produced by low energy neutrons.

For each target, measurements with the $1.27 \mathrm{~mm}$ diameter TEPC counter set in air approximately $85 \mathrm{~cm}$ from the target center at $0^{\circ}, 22^{\circ}, 45^{\circ}, 90^{\circ}$, and $142^{\circ}$ were made. These data, while not providing a great deal of quantitative information due to detector dead time problems, provide qualitative information about angular and atomic number dependences of the neutron procluction. Plots of event size spectra are shown in Figures 3.19-3.23. At $0^{\circ}$, spectra are dominated by high energy neutron events, so much so that events produced by low 


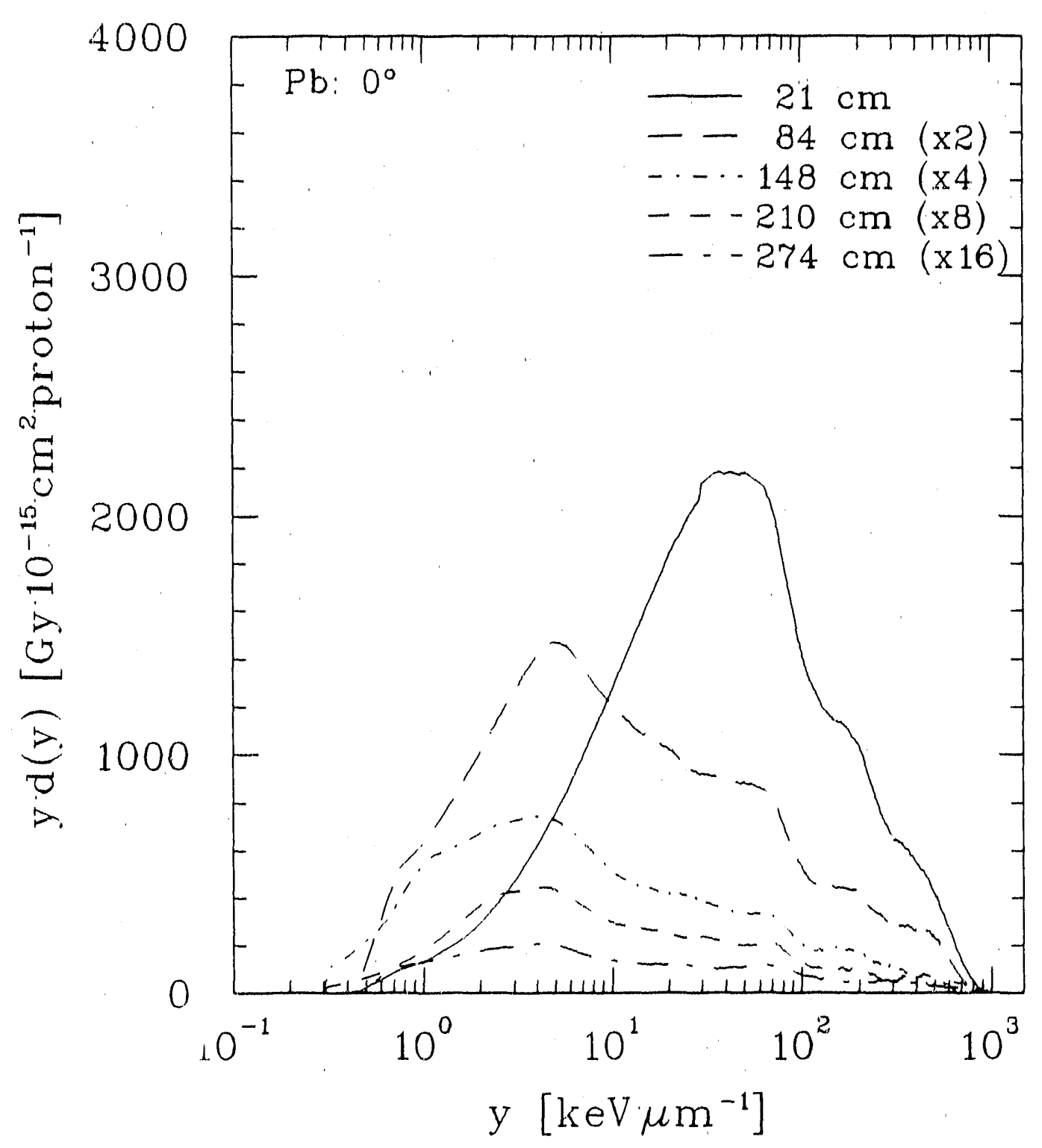

Figure 3.10: Event spectra observed at $0^{\circ}$ for each depth into the shield with $230 \mathrm{MeV}$ proton bombardment of a $\mathrm{Pb}$ target. 


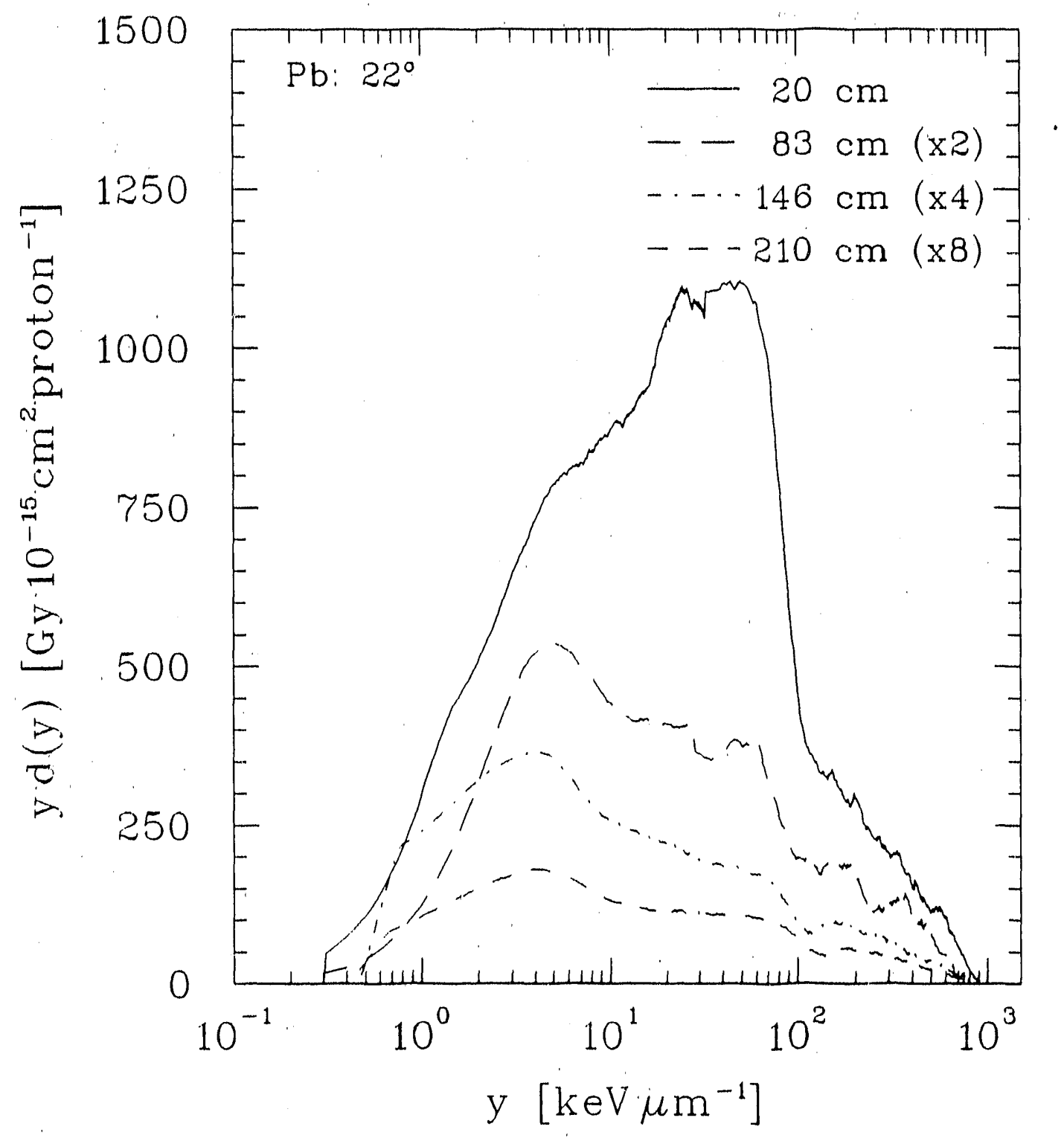

Figure 3.11: Event spectra observed at $22^{\circ}$ for multiple shield depths for $230 \mathrm{MeV}$ proton bombardment of a $\mathrm{Pb}$ target. 


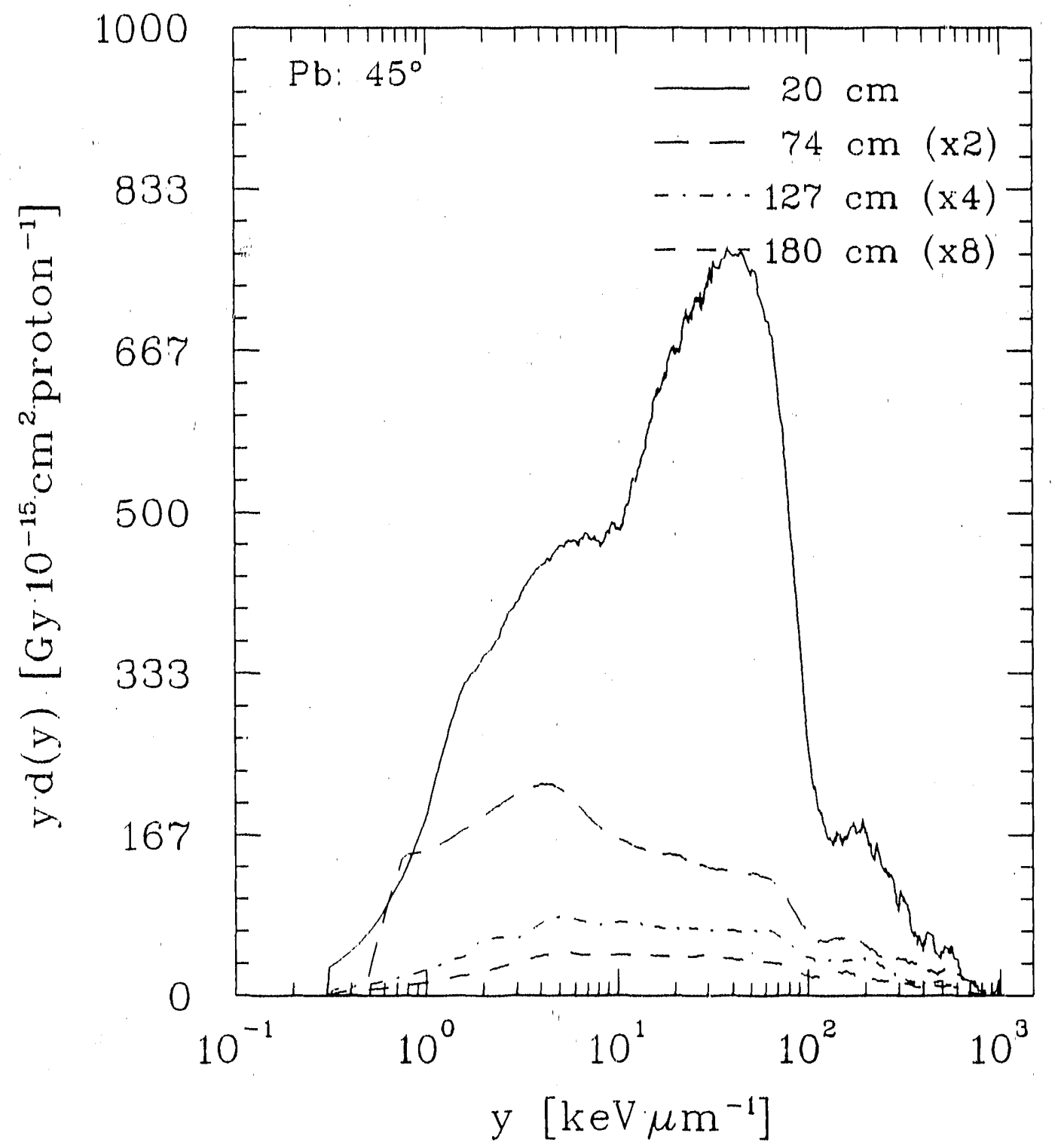

Figure 3.12: Event spectra observed at $45^{\circ}$ for multiple shield depths for $230 \mathrm{MeV}$ proton bombardment of a $\mathrm{Pb}$ target. 


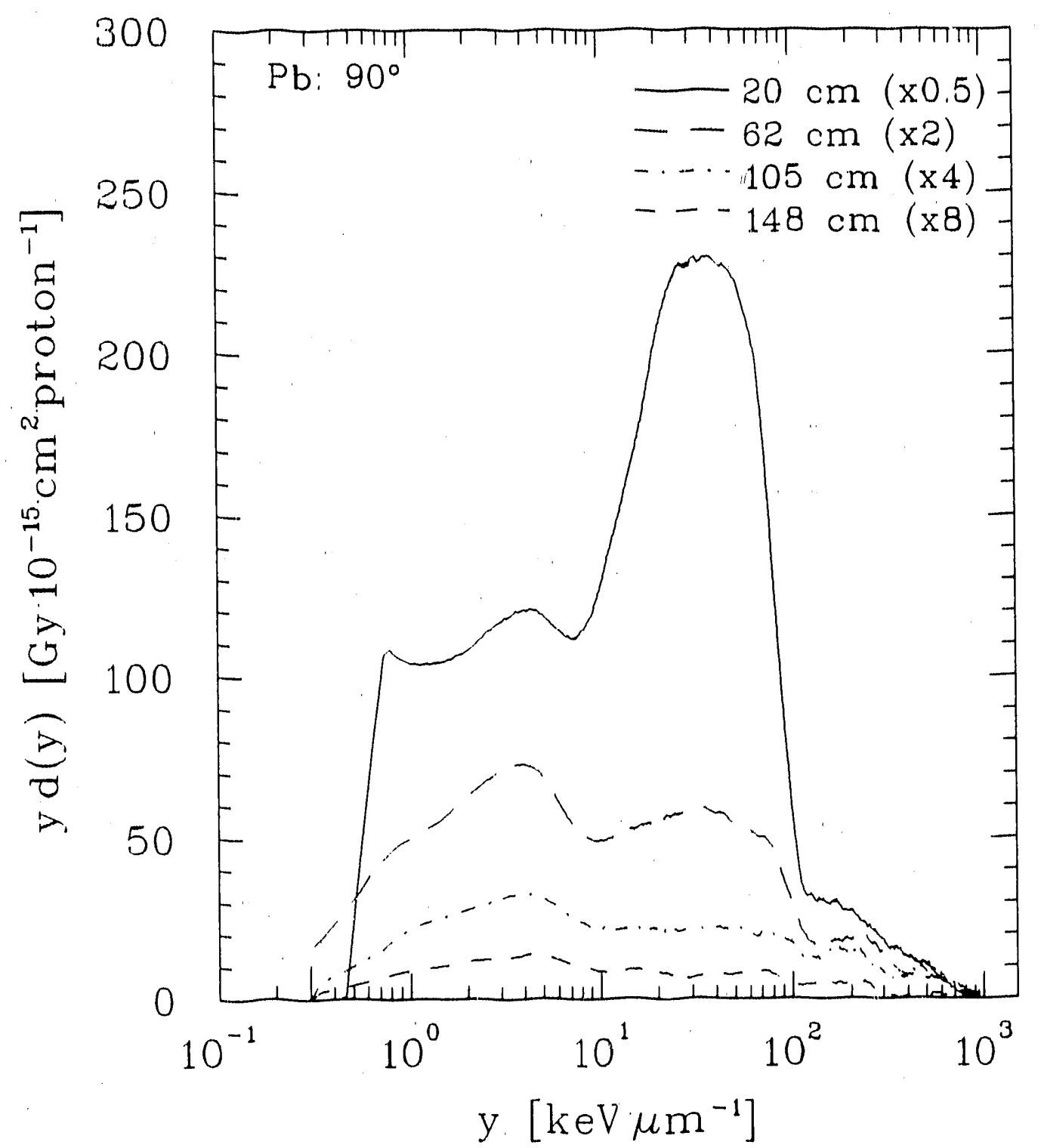

Figure 3.13: Event spectra observed at $90^{\circ}$ at multiple shield depths for $230 \mathrm{MeV}$ proton bombardment of a $\mathrm{Pb}$ target. Note, the $20 \mathrm{~cm}$ data has been halved in this display. 


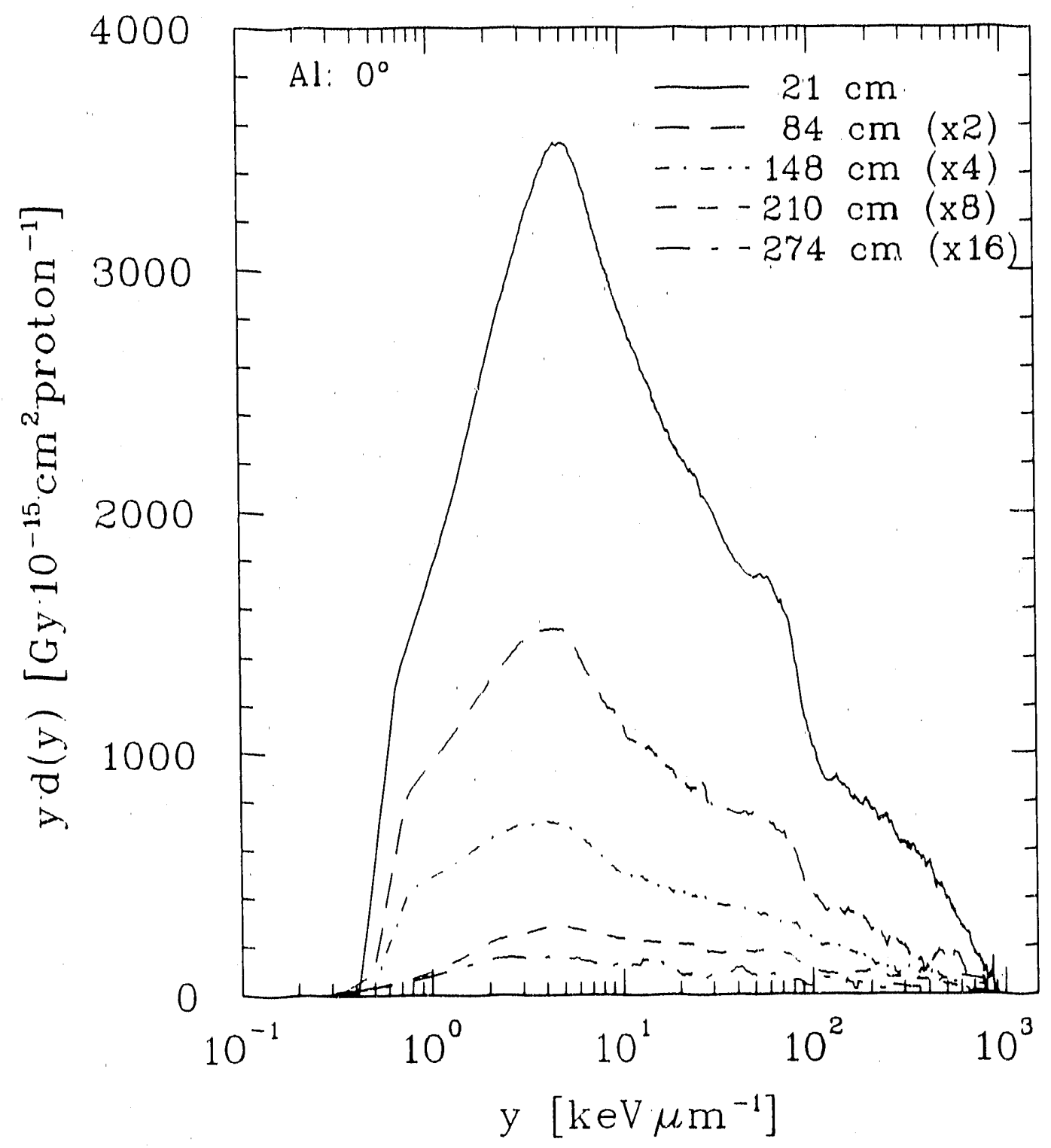

Figure 3.14: Event spectra observed at $0^{\circ}$ at multiple depths in the shield with 230 MeV protons incident upon Al target. 


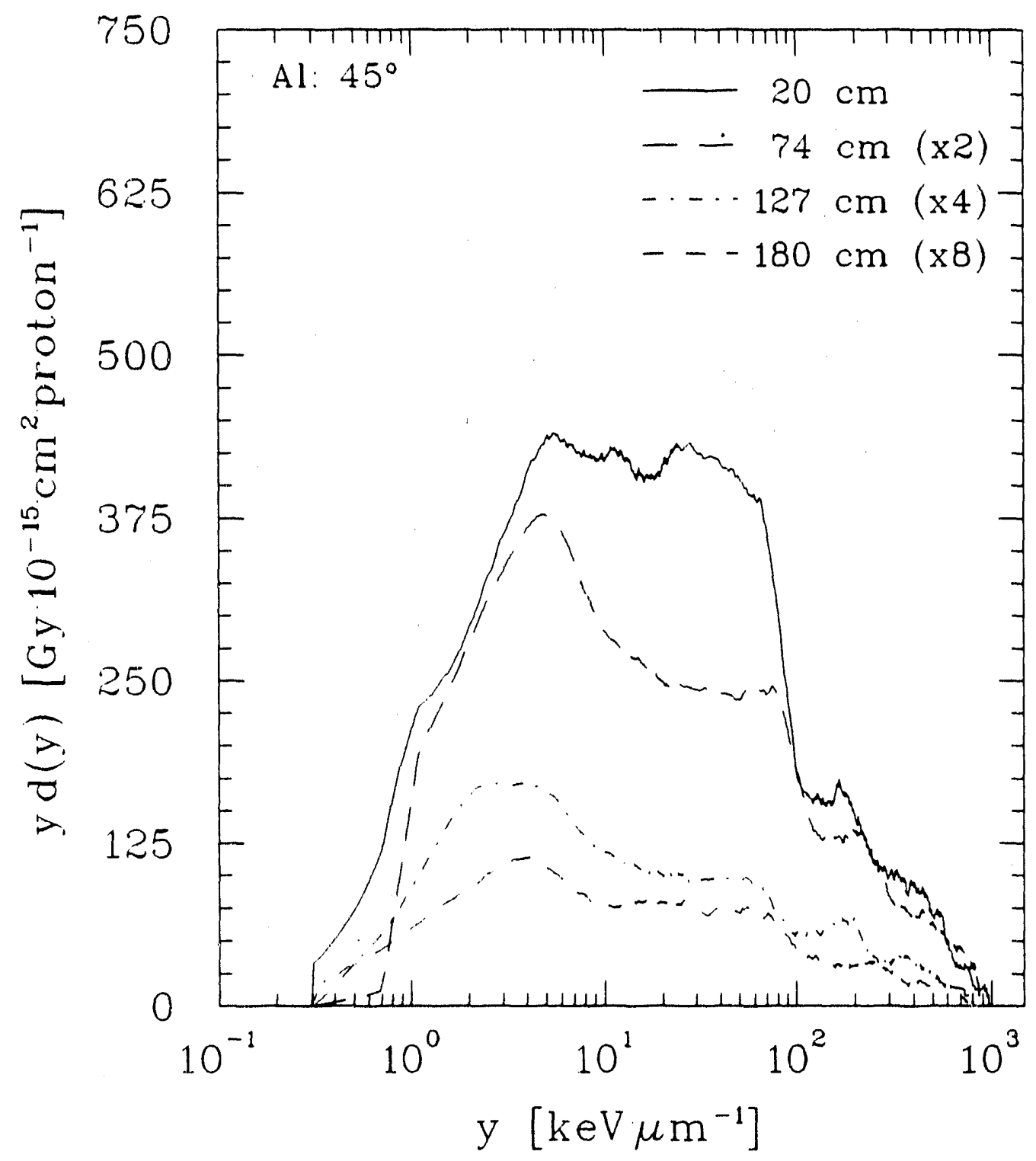

Figure 3.16: Event spectra observed at $45^{\circ}$ at multiple depths in the shield with $230 \mathrm{MeV}$ protons incident upon Al target. 


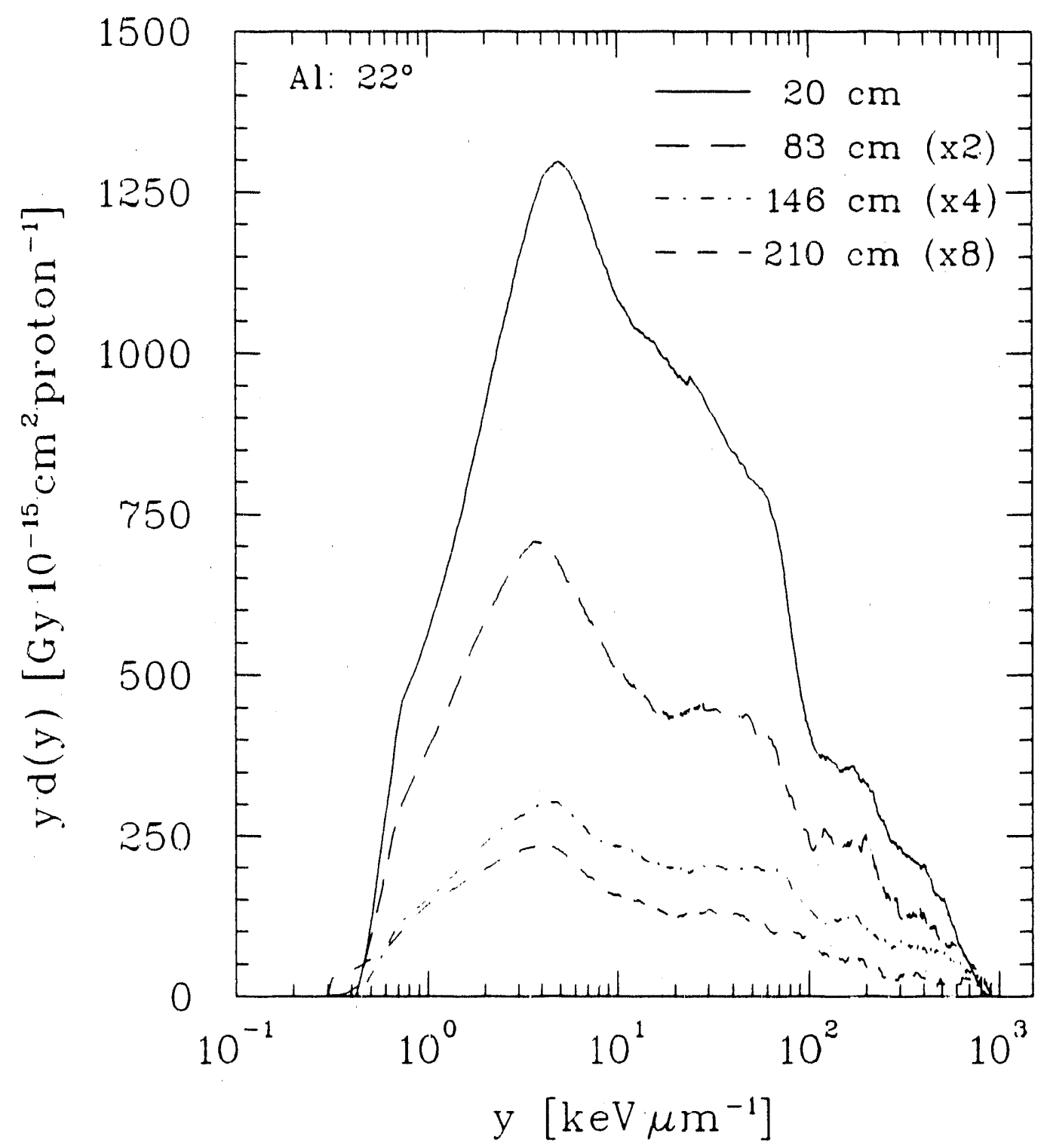

Figure 3.15: Event spectra observed at $22^{\circ}$ at multiple depths in the shield with $230 \mathrm{MeV}$ protons incident upon $\mathrm{Al}$ target. 


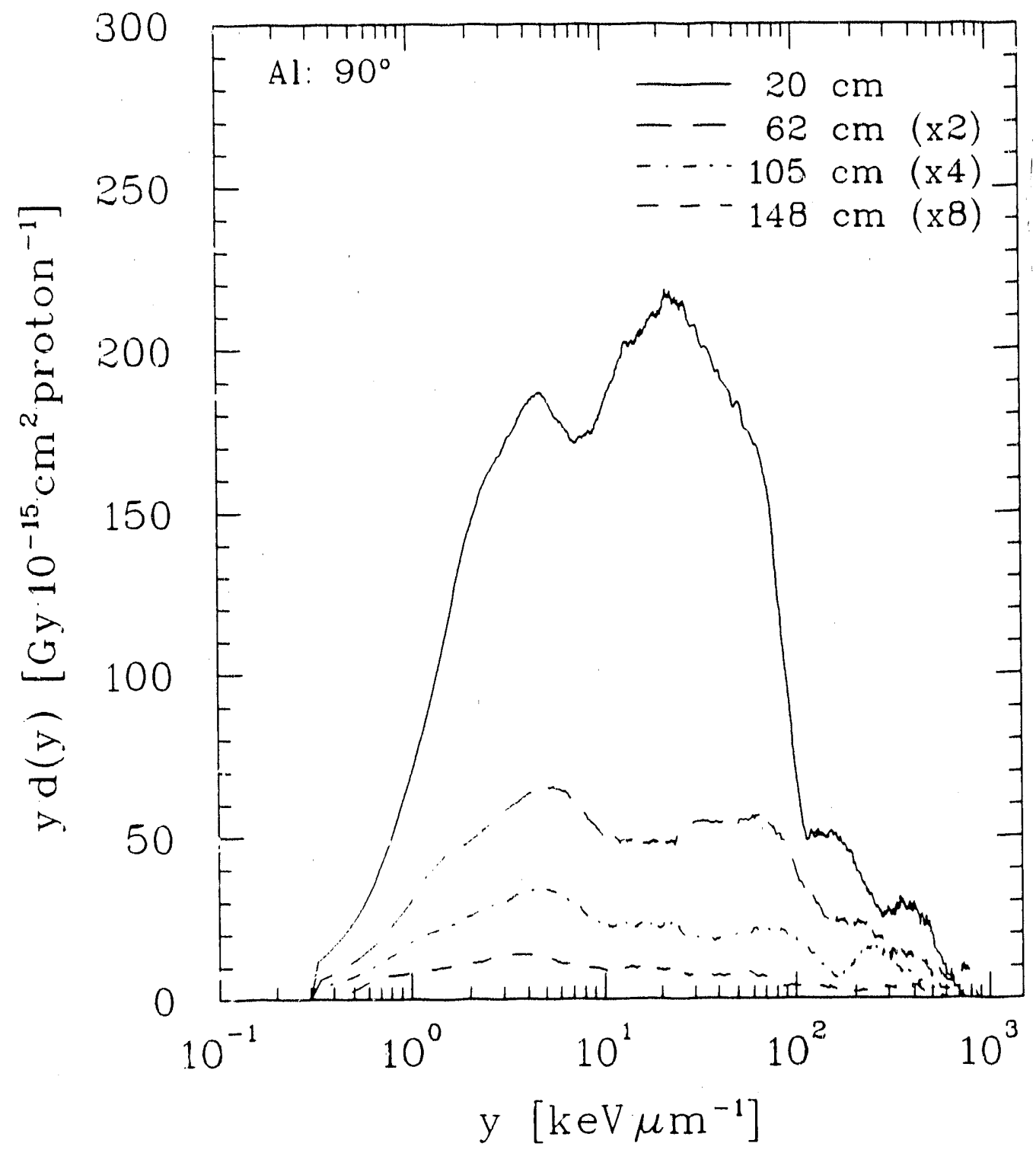

Figure 3.17: Eveni spectra observed at $90^{\circ}$ at multiple depths in the shield with $230 \mathrm{MeV}$ protons incidont upon A! target. 


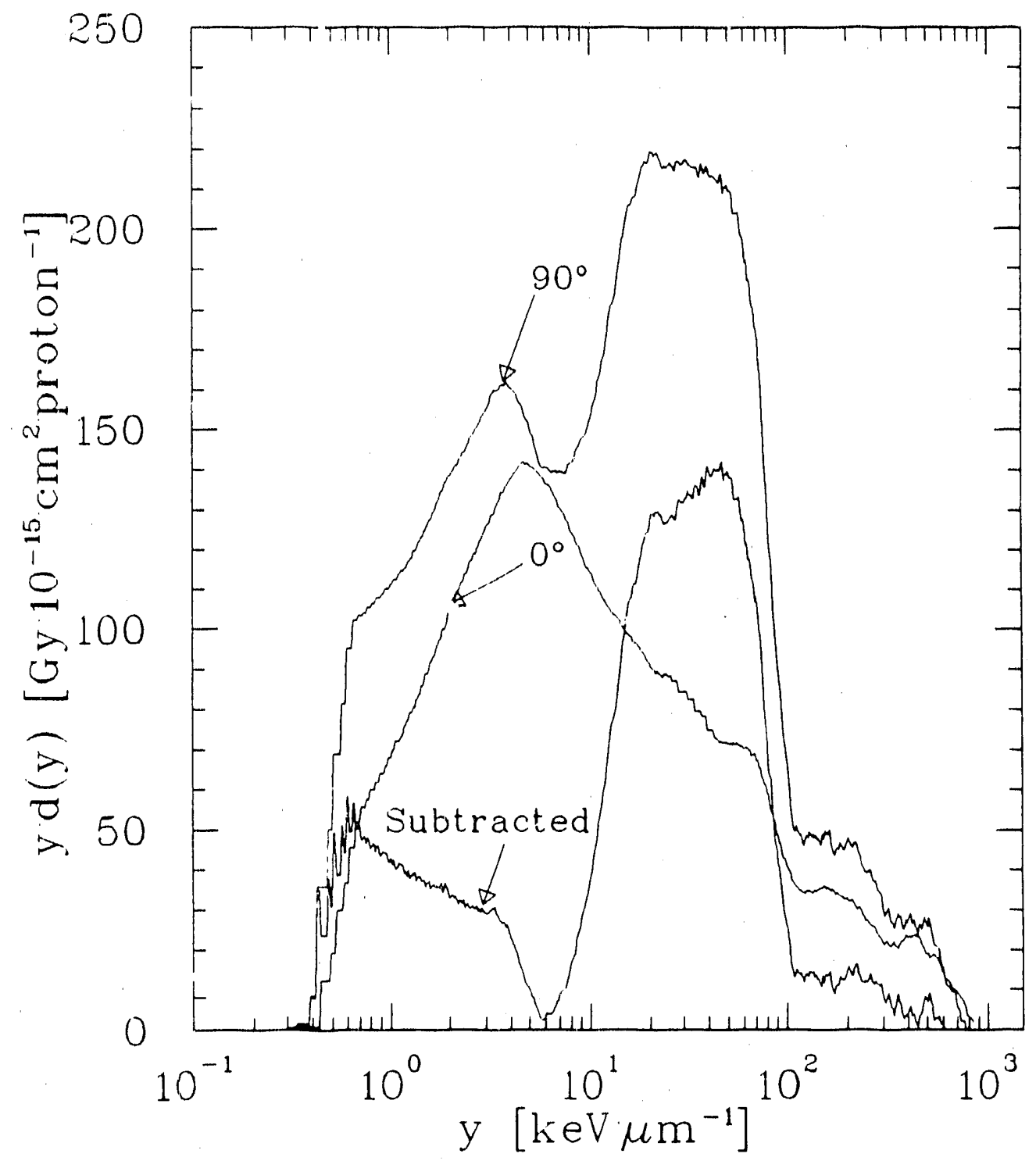

Figure 3.18: Event Spectrum obtained by subtracting a high energy neutron spectrum $\left(0^{\circ}, 84 \mathrm{~cm}\right)$ from the $90^{\circ}, 20 \mathrm{~cm}$ spectrum. The $0^{\circ}$ spectrum was normalized to the fast peak in the $90^{\circ}$ spectrum prior to subtraction. Residual gamma-ray spectrum and low energy neutrons are evident. 
energy neutrons are obscured. High energy neutrons dominate the Al spectra at $22^{\circ}$, the Fe data shows approximately equal low and high energy dose contributions, and low energy neutrons dominate the $\mathrm{Pb}$ data. Low energy neuirons begin to dominate the $\mathrm{Fe}$ data at $45^{\circ}$, however, for the $\mathrm{Al}$ target, high energy contributions persist. The $90^{\circ}$ and $142^{\circ}$ neutron emissions are dominated by low energy neutrons for the $\mathrm{Pb}$ and $\mathrm{Fe}$ targats, and the $\mathrm{Al}$ target shows very low dose values, indicating that few low energy neutrons being produced.

\subsection{Shielding Attenuation Curves}

Using Equation 2.18 the absorbed dose for each spectrum is determined. Mean quality factors and dose equivalent values are calculated using methods outlined in Section 2.5.6. Figures 3.24-3.26 plot dose equivalent values multiplied by the distance between the target and detector squared versus the thickness of concrete between the detector and target for each target material at $0^{\circ}, 22^{\circ}, 45^{\prime \prime}$, and $90^{\circ}$. Multiple data collection runs with a single target, angle, and depth are combined for plotting purposes.

Beyond $60 \mathrm{~cm}$ of penetration, exponential attenuation is observed. Before 30 $\mathrm{cm}$ penetration, low energy neutrons contribute a large fraction to the observed dose equivalent. These neutrons are rapidly attenuated from the spectrum at greater depths.

The exponential attenuation for a given target and angle combination is described by two parameters, the intercept (production or normalization term) and the slope (attenuation length). For our data set, the normaliation term 


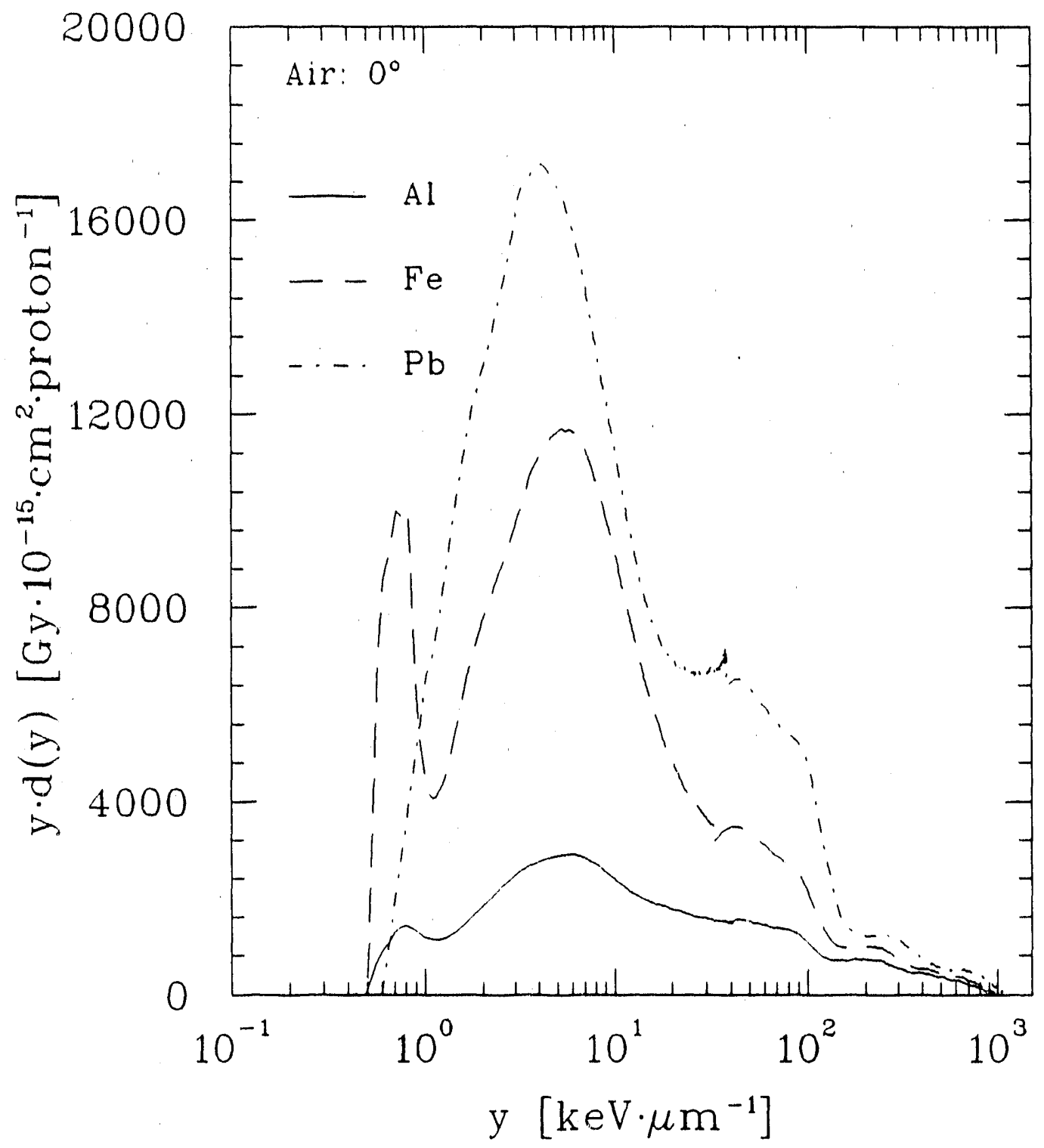

Figure 3.19: Event size spectrum measured in air at $0^{\circ}$ for each target material. 


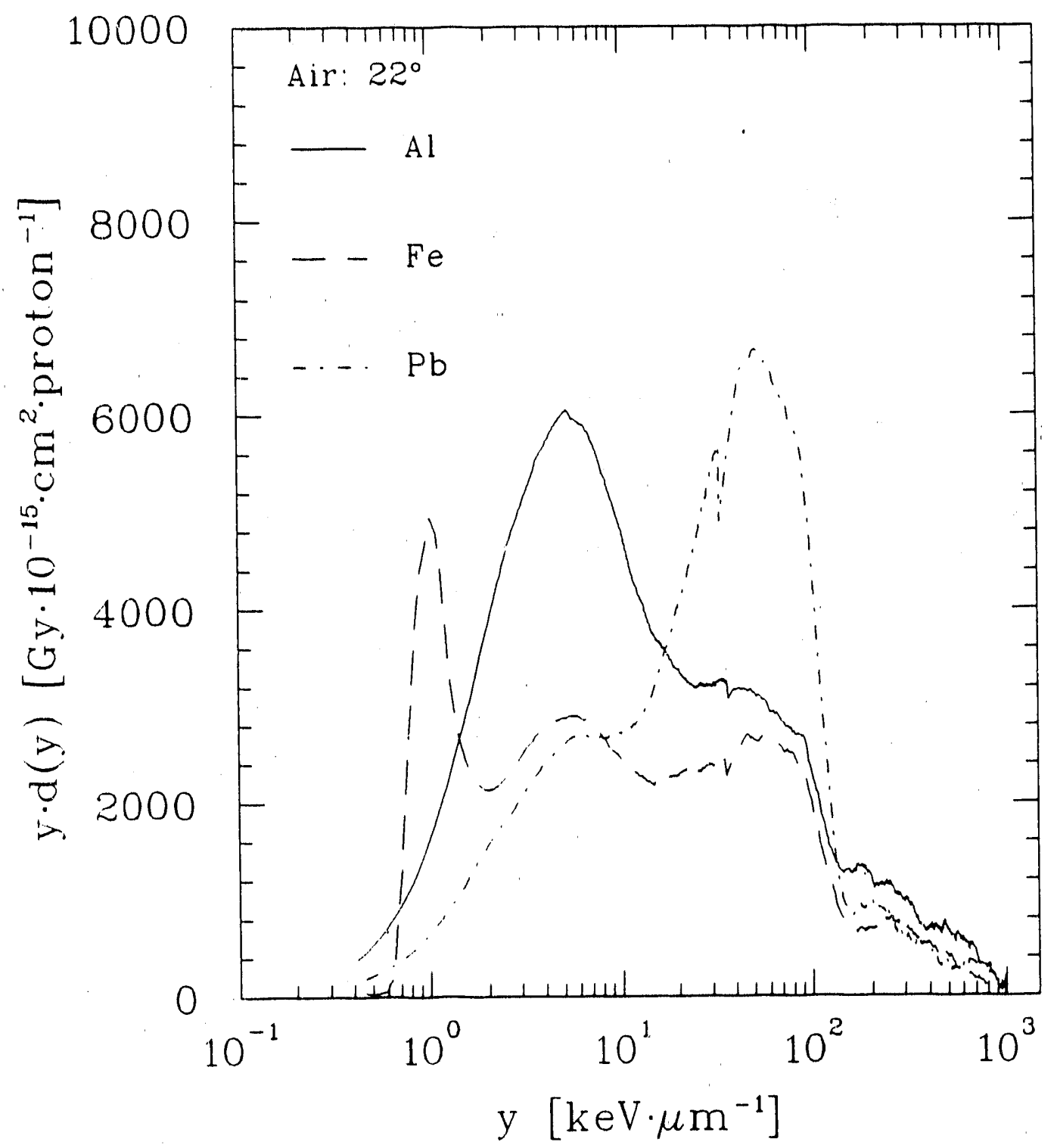

Figure 3.20: Event size spectrum measured in air at $22^{\circ}$ for each target material. 


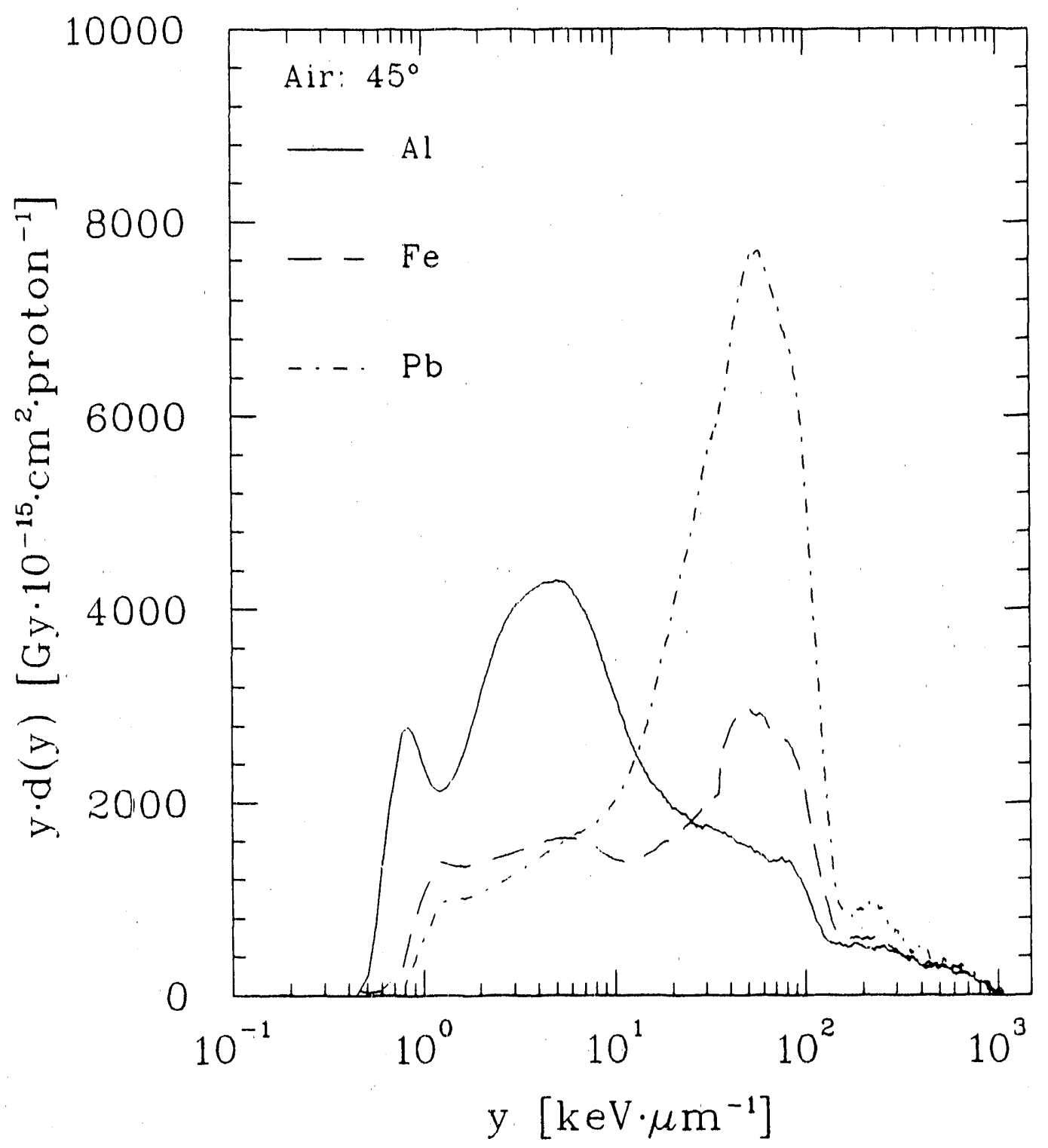

Figure 3.21: Event size spectrum measured in air at $45^{\circ}$ for each target material. 


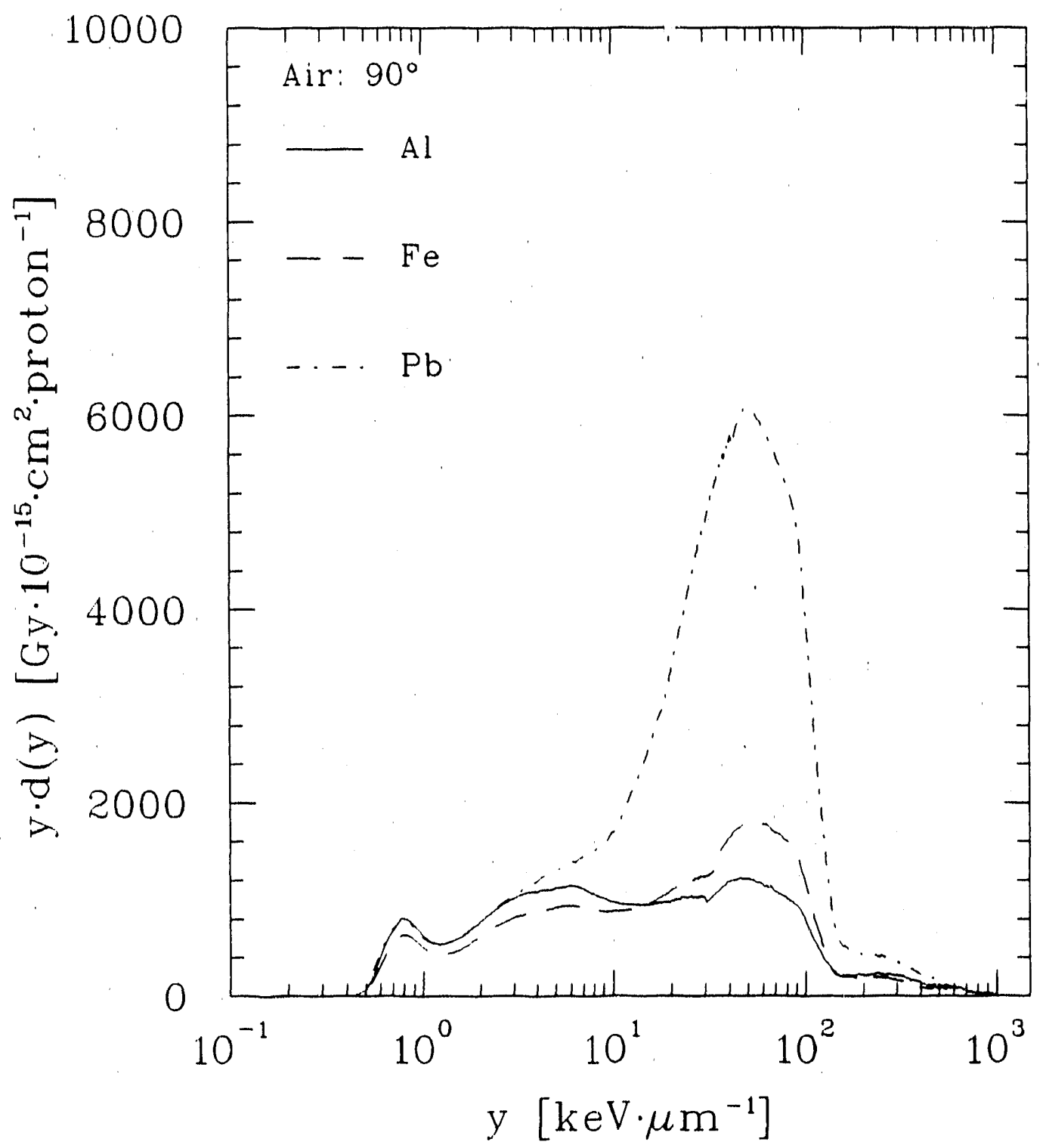

Figure 3.22: Event size spectrum measured in air at $90^{\circ}$ for each target material. 


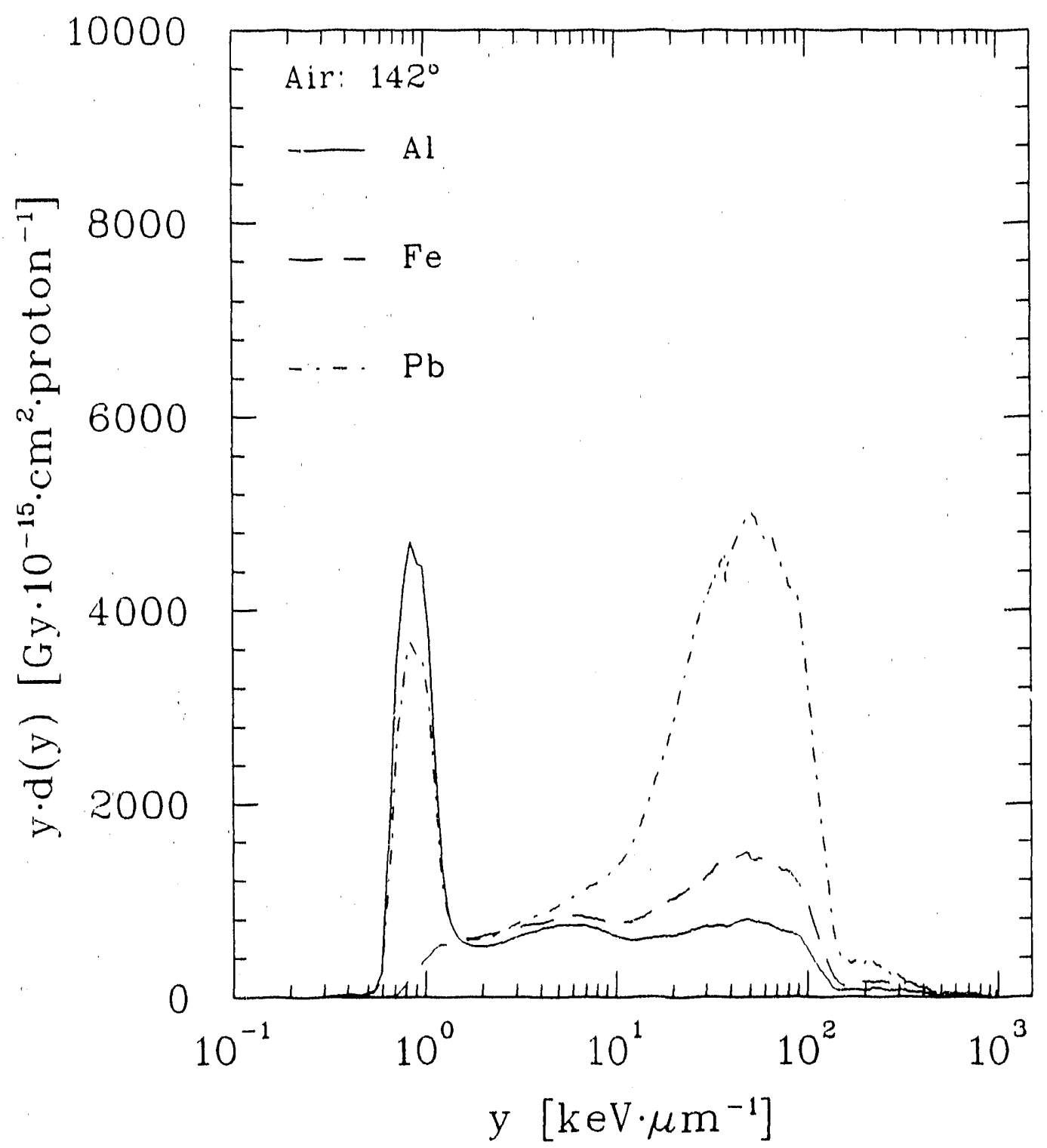

Figure 3.23: Event size spectrum measured in air at $142^{\circ}$ for each target material. 


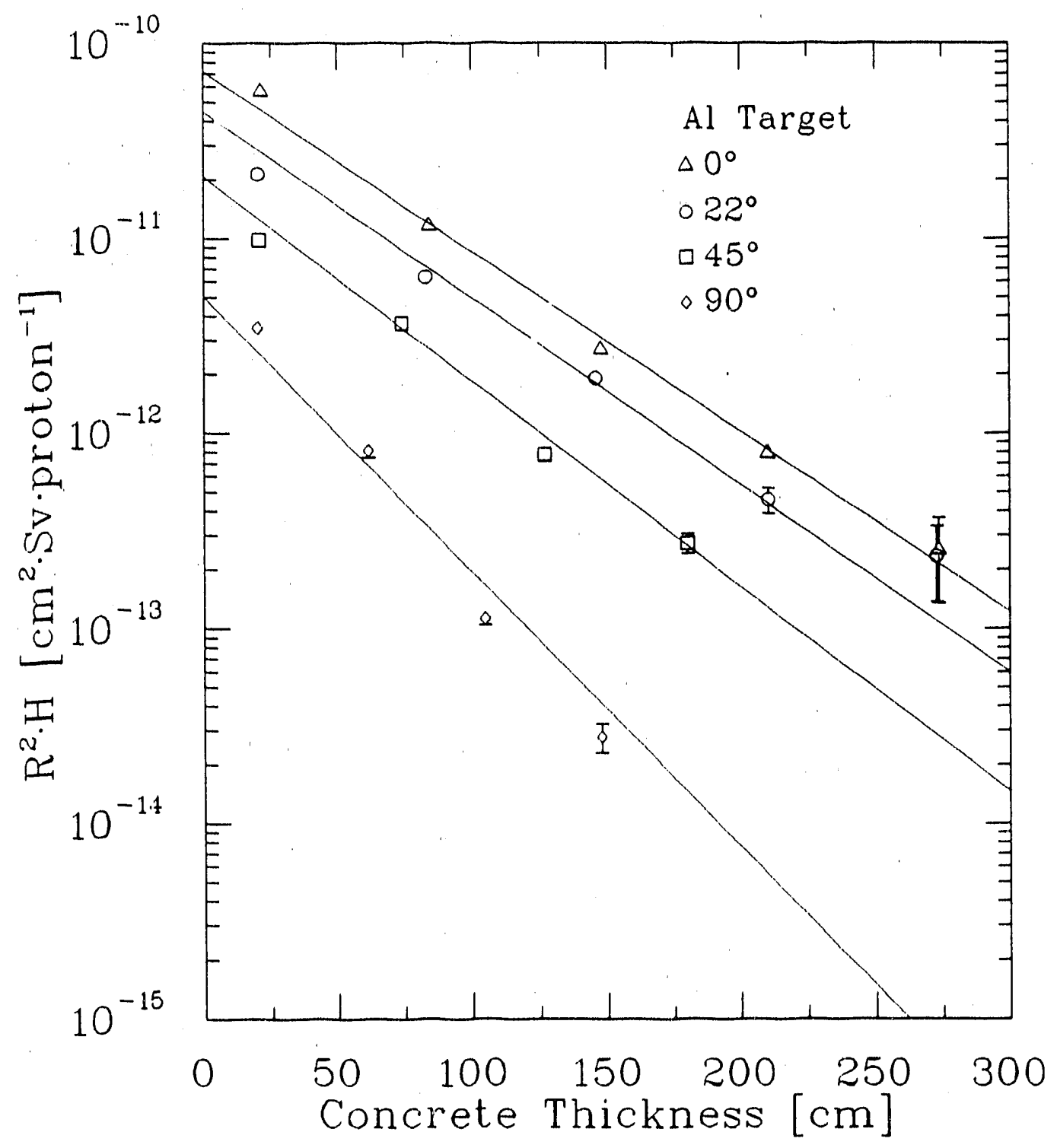

Figure 3.24: Measured dose equivalent multiplied by the distance between the detector center and target squared versus concrete thickness for $2.30 \mathrm{MeV}$ proton bombardment of a stopping length $\mathrm{Al}$ target. 


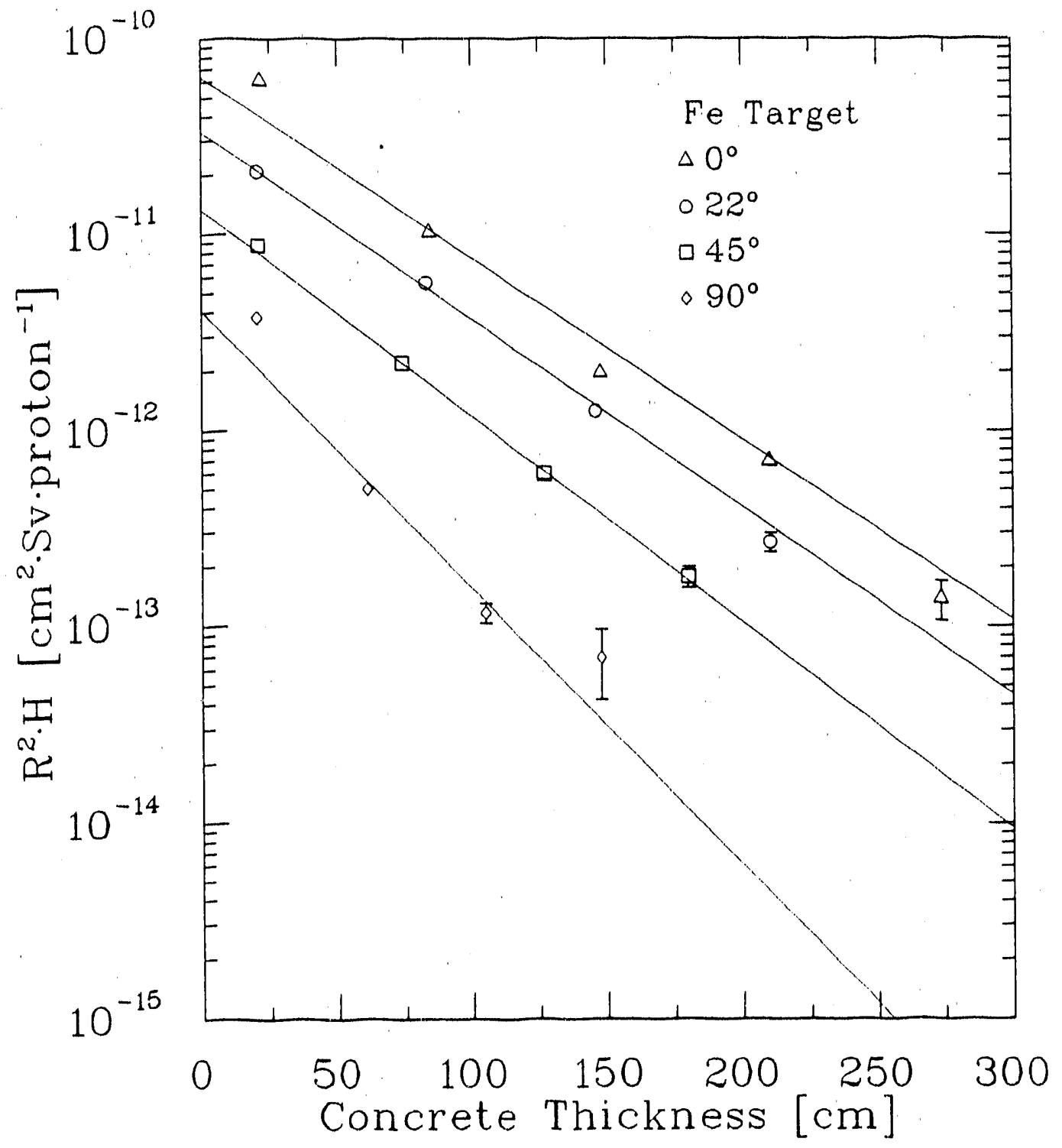

Figure 3.25: Mcasured dose equivalent multiplied by the distance between the detector center and target squared versus concrete thickness for $230 \mathrm{MeV}$ proton bombardment of a stopping length $\mathrm{Fe}$ target. 


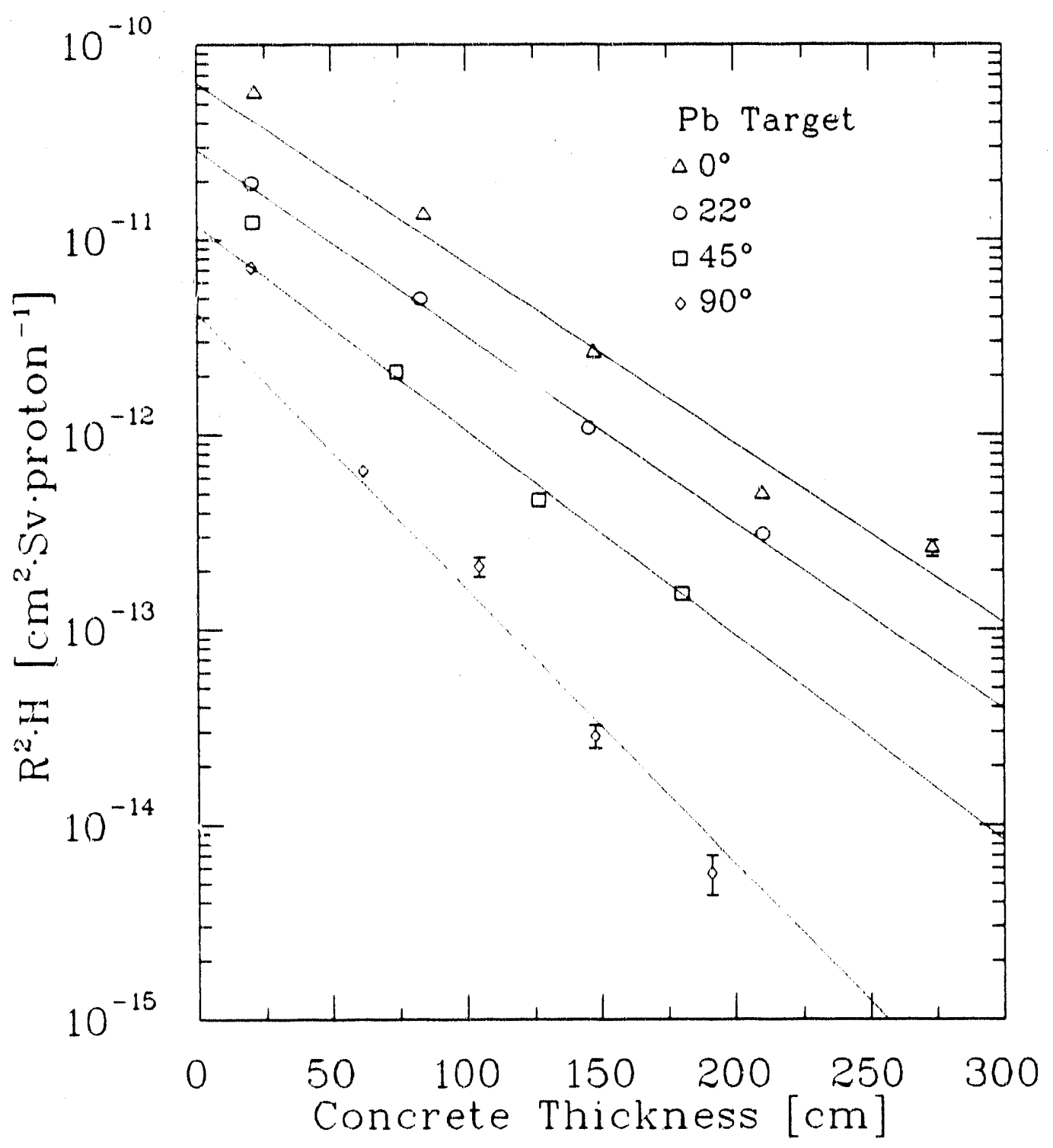

Figure 3.26: Measured dose equivalent multiplied by the distance between the detector center and target squared versus concrete thickness for $230 \mathrm{MeV}$ proton bombardment of a stopping length $\mathrm{Pb}$ target. 
describes the dose equivalent evaluated when shield thickness is extrapolated to zero. This value specifies the "source term" of the component that is being exponentially attenuated. The attenuation length describes the dose equivalent falloff with shield thickness.

Dose equivalent attenuation parameters are determined using a weighted least. squares fit to the form

$$
r^{2} H=P e^{-\frac{d}{\lambda}},
$$

where $r$ is the distance between the detector and target, $H$ is the dose equivalent at concrete depth $d, \lambda$ is the attenuation length, and $P$ is the normalization constant. The weights for each data point $i$ are chosen to be equal to $\sigma_{r^{2} H_{i}}^{-2}$. Due to the hardening of the observed spectra, fits are done only to penetrations greater than $59 \mathrm{~cm}\left(110 \mathrm{~g} \cdot \mathrm{cm}^{-2}\right)$. Some additional spectral hardening occurs after $59 \mathrm{~cm}$, but this effect is minimal. Table 3.1 summarizes the determined attenuation parameters. Beyond the first penetration, at each angle, dose equivalent values are largely target independent as shown in Figure 3.27.

Due to the similarity in event size data at each angle and depth for the different target materials, data are also fitted with a common attenuation length allowing different intercepts for each target. Fitting to a common slope is accomplished using a weighted least squares fit to the set of $n$ equations

$$
r^{2} H_{n}=\left(a P_{A l}+b P_{F e}+c P_{P b}\right)-c^{-\frac{d}{r}}
$$

where $P_{A l}$. $P_{F e}$, and $P_{P b}$ are the intercepts for the Al. Fe, and $P$ l targets respectively, and $a, b$, and $c$ are boolean operators signaling the target used in measurement $n$. For example, for a measurement with the Fe target $a=0$, 


\begin{tabular}{|c|c|c|c|c|}
\hline \multirow[b]{2}{*}{ Angle } & \multicolumn{2}{|r|}{ Single Target } & \multicolumn{2}{|r|}{ All Targets } \\
\hline & $\begin{array}{l}\text { Slope } \\
(\mathrm{cm})\end{array}$ & $\begin{array}{c}\text { Intercept } \\
\left(\mathrm{cm}^{2} \cdot \mathrm{pSv} \cdot \text { proton }^{-1}\right)\end{array}$ & $\begin{array}{l}\text { Slope } \\
(\mathrm{cm})\end{array}$ & $\begin{array}{c}\text { Intercept } \\
\left(\mathrm{cm}^{2} \cdot \mathrm{pSv} \cdot \text { proton }^{-1}\right)\end{array}$ \\
\hline \multicolumn{5}{|c|}{ Al Target } \\
\hline 0 & $50.0 \pm 2.4$ & $60 \pm 9$ & $47.1 \pm 1.5$ & $\tau 2 \pm 9$ \\
\hline 22 & $49.8 \pm 1.6$ & $35 \pm 3$ & $45.4 \pm 1.6$ & $45 \pm 5$ \\
\hline 45 & $40 \pm 4$ & $23 \pm 6$ & $41.3 \pm 1.3$ & $20.7 \pm 2.0$ \\
\hline 90 & $31 \pm 5$ & $5.0 \pm 2.7$ & $30.8 \pm 2.2$ & $5.0 \pm 1.3$ \\
\hline \multicolumn{5}{|c|}{ Fe Target } \\
\hline 0 & $45.7 \pm 1.4$ & $70 \pm 7$ & $47.1 \pm 1.5$ & $64 \pm 7$ \\
\hline 22 & $43.1 \pm 1.6$ & $38 \pm 4$ & $45.4 \pm 1.6$ & $34 \pm 3$ \\
\hline 45 & $41.9 \pm 0.5$ & $12.9 \pm 0.4$ & $41.3 \pm 1.3$ & $1.3 .4 \pm 1.2$ \\
\hline 90 & $39 \pm 5$ & $2.2 \pm 0.7$ & $30.8 \pm 2.2$ & $4.1 \pm 1.0$ \\
\hline \multicolumn{5}{|c|}{$\mathrm{Pb}$ Target } \\
\hline 0 & $45 \pm 5$ & $76 \pm 31$ & $47.1 \pm 1.5$ & $64 \pm 9$ \\
\hline 22 & $44 \pm 4$ & $31 \pm 8$ & $45.4 \pm 1.6$ & $29 \pm 4$ \\
\hline 45 & $42.3 \pm 2.2$ & $11.0 \pm 1.7$ & $41.3 \pm 1.3$ & $11.8 \pm 1.3$ \\
\hline 90 & $26.9 \pm 1.4$ & $7.7 \pm 2.1$ & $30.8 \pm 2.2$ & $4.2 \pm 1.5$ \\
\hline
\end{tabular}

Table 3.1: Parameters for exponential attenuation fits to dose equivalent in concrete shield $\left(\rho=1.88 \mathrm{~g} \cdot \mathrm{cm}^{-2}\right)$. Fits are performed on each target separately (Single Target) and using a common slope, allowing for different normalization terms for each target. (All Targets). 


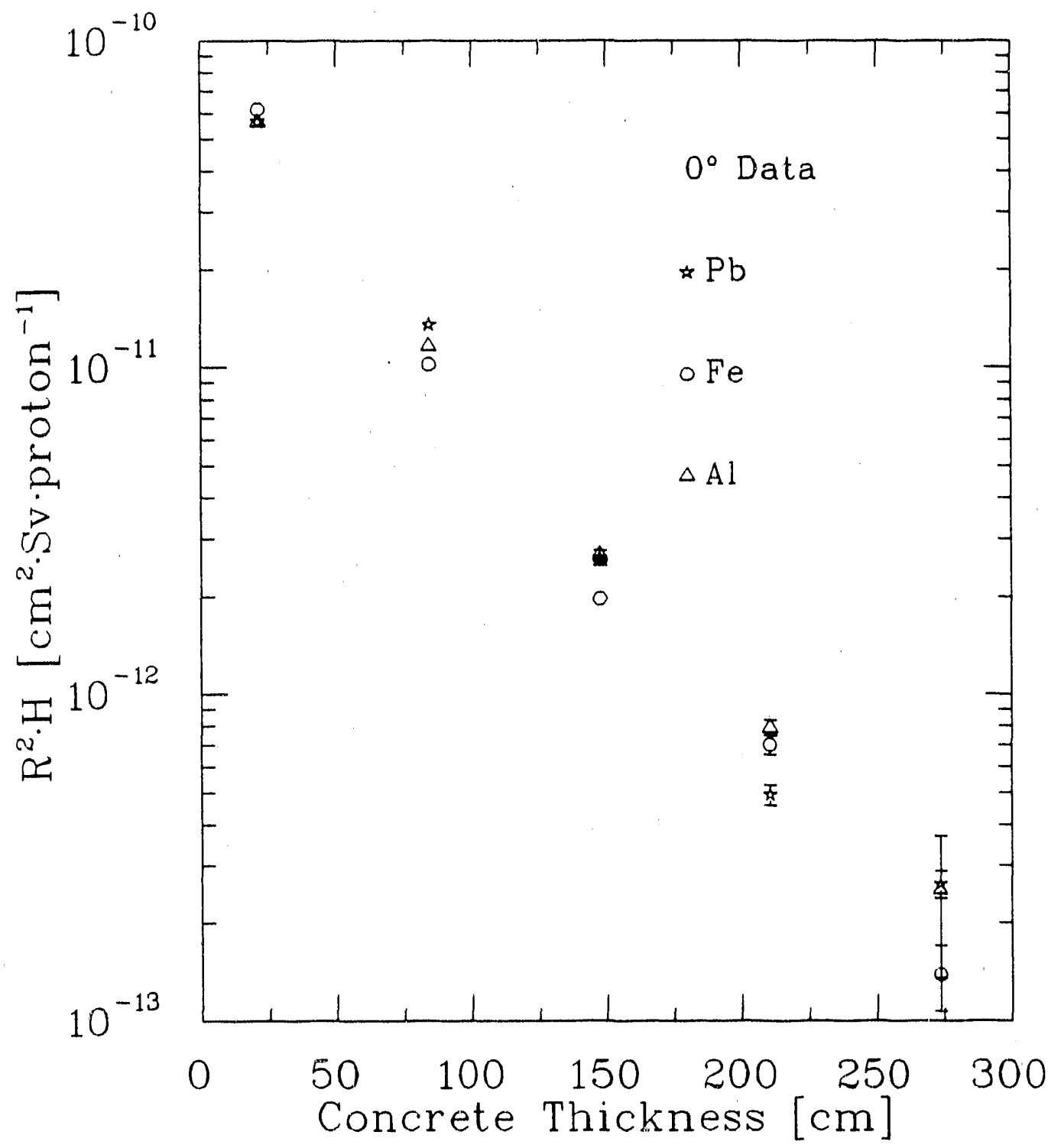

Figure 3.27: Dose equivalent values observed at $0^{\circ}$ for all target materials. Similarity in values justifies fitting data to a common attenuation length. 
$b=1$, and $c=0$. Values computed using this method are summarized in Table 3.1, and are plotted with the data in Figures 3.24-3.26.

Dose equivalent is not a directly measurable quantity since it relies on the use of quality factors which are defined or legislated quantities. In these measurements, absorbed dose is measured and radiation quality determined using a proposed event size-rudiation quality relationship. This relationship (Equation 2.19 ), is only one of several recommended procedures. Since the defining relationships between event size and radiation quality may change, absorbed dose and radiation quality factors are given in Tables 3.2-3.4 for each target and angle to allow comparison of our results with others. 


\begin{tabular}{|c|c|c|c|}
\hline $\begin{array}{l}\text { Depth } \\
(\mathrm{cm}) \\
\end{array}$ & $\begin{array}{c}\mathrm{H} \\
\left(\mathrm{pSv} \cdot \mathrm{cm}^{2} \cdot \text { Proton }^{-1}\right)\end{array}$ & $\begin{array}{c}\text { D } \\
\left(\mathrm{pGy} \cdot \mathrm{cm}^{2} \cdot \text { Proton }^{-1}\right)\end{array}$ & $\mathrm{Q}$ \\
\hline $\mathrm{Al} 0^{\circ}$ & & & \\
\hline 21.4 & $56.5 \pm 1.2$ & $8.67 \pm 0.03$ & $6.51 \pm 0.16$ \\
\hline 84.3 & $11.6 \pm 0.2$ & $1.92 \pm 0.01$ & $5.89 \pm 0.11$ \\
\hline 147.8 & $2.68 \pm 0.12$ & $0.453 \pm 0.003$ & $6.1 \pm 0.3$ \\
\hline 210.0 & $0.79 \pm 0.04$ & $0.109 \pm 0.001$ & $7.6 \pm 0.4$ \\
\hline 273.5 & $0.25 \pm 0.12$ & $0.026 \pm 0.002$ & $9.7 \pm 4.4$ \\
\hline $\mathrm{Al} 22^{\circ}$ & & & \\
\hline 20.3 & $21.3 \pm 0.4$ & $3.31 \pm 0.01$ & $6.41 \pm 0.12$ \\
\hline 83.0 & $6.3 \pm 0.3$ & $0.976 \pm 0.005$ & $6.4 \pm 0.3$ \\
\hline 145.8 & $1.90 \pm 0.09$ & $0.274 \pm 0.00^{\circ}$ & $6.9 \pm 0 . .3$ \\
\hline 210.2 & $0.46 \pm 0.07$ & $0.072 \pm 0.001$ & $6.3 \pm 0.9$ \\
\hline 272.6 & $0.24 \pm 0.10$ & $0.026 \pm 0.002$ & $9.0 \pm 3.7$ \\
\hline $\mathrm{Al} 45^{\circ}$ & & & \\
\hline 20.3 & $9.8 \pm 0.2$ & $1.344 \pm 0.004$ & $7.30 \pm 0.1 \%$ \\
\hline 74.1 & $3.67 \pm 0.11$ & $0.470 \pm 0.002$ & $7.91 \pm 0.23$ \\
\hline 126.9 & $0.78 \pm 0.06$ & $0.105 \pm 0.001$ & $7.4 \pm 0.6$ \\
\hline 180.1 & $0.27 \pm 0.03$ & $0.0375 \pm 0.0006$ & $7.3 \pm 0.8$ \\
\hline Al $90^{\circ}$ & & & \\
\hline 20.0 & $3.47 \pm 0.10$ & $0.572 \pm 0.002$ & $6.05 \pm 0.17$ \\
\hline 61.5 & $0.81 \pm 0.06$ & $0.106 \pm 0.001$ & $7.7 \pm 0.6$ \\
\hline 104.8 & $0.113 \pm 0.009$ & $0.0190 \pm 0.0002$ & $6.1 \pm 0.5$ \\
\hline 147.8 & $0.028 \pm 0.005$ & $0.0044 \pm 0.0001$ & $6.2 \pm 1.1$ \\
\hline
\end{tabular}

Table 3.2: Dose equivalent, absorbed dose, and quality factors measured at depth and angle in a concrete shield for $230 \mathrm{MeV}$ protons incident upon an Al target. 


\begin{tabular}{|c|c|c|c|}
\hline $\begin{array}{l}\text { Depth } \\
(\mathrm{cm}) \\
\end{array}$ & $\begin{array}{c}\mathrm{H} \\
\left(\mathrm{pSv} \cdot \mathrm{cm}^{2} \cdot \text { Proton }^{-1}\right)\end{array}$ & $\begin{array}{c}D \\
\left(\mathrm{pG} \cdot \mathrm{cm}^{2} \cdot \text { Proton }^{-1}\right)\end{array}$ & $Q$ \\
\hline $\mathrm{Fe} 0^{\circ}$ & & & \\
\hline 21.4 & $61.6 \pm 1.4$ & $10.23 \pm 0.03$ & $6.03 \pm 0.13$ \\
\hline 84.3 & 10.2 上 0.3 & $1.788 \pm 0.006$ & $5.75 \pm 0.17$ \\
\hline 147.8 & $3.04 \pm 0.13$ & $0.510 \pm 0.002$ & $6.0 \pm 0 . .6$ \\
\hline 210.0 & $0.70 \pm 0.05$ & $0.116 \pm 0.001$ & $6.2 \pm 0.4$ \\
\hline 273.5 & $0.14 \pm 0.03$ & $0.0169 \pm 0.0007$ & $8.2 \pm 1.8$ \\
\hline $\mathrm{Fe} 22^{\circ}$ & & & \\
\hline 20.3 & $21.0 \pm 0.4$ & $3.06 \pm 0.01$ & $6.89 \pm 0.12$ \\
\hline 83.0 & $5.64 \pm 0.17$ & $0.911 \pm 0.004$ & $5.87 \pm 0.17$ \\
\hline 145.8 & $1.26 \pm 0.06$ & $0.195 \pm 0.001$ & $6.6 \pm 0.3$ \\
\hline 210.2 & $0.2 \uparrow \pm 0.03$ & $0.0 .379 \pm 0.0006$ & $7.1 \pm 0.8$ \\
\hline $\mathrm{Fe} 45^{\circ}$ & & & \\
\hline 20.3 & $8.81 \pm 0.22$ & $1.312 \pm 0.005$ & $6.68 \pm 0.15$ \\
\hline 74.1 & $2.22 \pm 0.06$ & $0.288 \pm 0.001$ & $T .72 \pm 0.22$ \\
\hline 126.9 & $0.61 \pm 0.05$ & $0.0932 \pm 0.0012$ & $6.5 \pm 0.5$ \\
\hline 180.1 & $0.179 \pm 0.022$ & $0.0268 \pm 0.0004$ & $6.7 \pm 0.8$ \\
\hline $\mathrm{Fe} 90^{\circ}$ & & & \\
\hline 20.0 & $3.78 \pm 0.08$ & $0.612 \pm 0.003$ & $6.07 \pm 0.12$ \\
\hline 61.5 & $0.503 \pm 0.022$ & $0.0747 \pm 0.0005$ & $6.7 \pm 0.3$ \\
\hline 104.8 & $0.118 \pm 0.014$ & $0.0169 \pm 0.0003$ & $6.9 \pm 0.8$ \\
\hline 147.8 & $0.07 \pm 0.03$ & $0.0304 \pm 0 .(1,03$ & $2.3 \pm 3.0$ \\
\hline
\end{tabular}

Table 3.3: Dose equivalent, absorbed dose, and quality factors measured at depth and angle in a concrete shield for $230 \mathrm{MeV}$ protons incident upon an Fe larget. 


\begin{tabular}{|l|c|c|c|}
\hline $\begin{array}{l}\text { Depth } \\
(\mathrm{cm})\end{array}$ & $\begin{array}{c}\mathrm{H} \\
\left(\mathrm{pSv} \cdot \mathrm{cm}^{2} \cdot \text { Proton }^{-1}\right)\end{array}$ & $\begin{array}{c}\mathrm{D} \\
\left(\mathrm{pGy} \cdot \mathrm{cm}^{2} \cdot \text { Proton }^{-1}\right)\end{array}$ & $\mathrm{Q}$ \\
\hline $\mathrm{Pb} 0^{\circ}$ & & & \\
21.4 & $56.5 \pm 0.9$ & $4.96 \pm 0.02$ & $11.40 \pm 0.13$ \\
84.3 & $13.5 \pm 0.3$ & $1.98 \pm 0.01$ & $6.79 \pm 0.14$ \\
147.8 & $2.63 \pm 0.14$ & $0.323 \pm 0.001$ & $6.5 \pm 0.4$ \\
210.0 & $0.49 \pm 0.04$ & $0.0724 \pm 0.0007$ & $6.4 \pm 0.4$ \\
273.5 & $0.262 \pm 0.025$ & $0.0367 \pm 0.0004$ & $7.2 \pm 0.7$ \\
& & & \\
$\mathrm{~Pb} 22^{\circ}$ & & & \\
20.3 & $19.7 \pm 0.3$ & $2.255 \pm 0.007$ & $8.11 \pm 0.12$ \\
83.0 & $4.98 \pm 0.17$ & $0.731 \pm 0.003$ & $6.83 \pm 0.22$ \\
145.8 & $1.08 \pm 0.04$ & $0.1515 \pm 0.0008$ & $6.72 \pm 0.22$ \\
210.2 & $0.311 \pm 0.018$ & $0.0513 \pm 0.0004$ & $6.5 \pm 0.3$ \\
& & & \\
$\mathrm{~Pb} 45^{\circ}$ & & & \\
20.3 & $12.3 \pm 0.3$ & $1.89 \pm 0.01$ & $6.54 \pm 0.15$ \\
74.1 & $2.11 \pm 0.02$ & $0.319 \pm 0.001$ & $6.76 \pm 0.03$ \\
126.9 & $0.463 \pm 0.026$ & $0.0620 \pm 0.0006$ & $7.5 \pm 0.4$ \\
180.1 & $0.153 \pm 0.011$ & $0.0185 \pm 0.0002$ & $8.2 \pm 0.6$ \\
& & & \\
$\mathrm{~Pb} 90^{\circ}$ & & & \\
20.0 & $7.19 \pm 0.16$ & $1.292 \pm 0.004$ & $5.56 \pm 0.11$ \\
61.5 & $0.656 \pm 0.026$ & $0.1138 \pm 0.0005$ & $5.76 \pm 0.22$ \\
104.8 & $0.211 \pm 0.025$ & $0.0253 \pm 0.0004$ & $8.4 \pm 1.0$ \\
147.8 & $0.0284 \pm 0.004$ & $0.0047 \pm 0.0001$ & $6.1 \pm 0.8$ \\
190.8 & $0.0056 \pm 0.0013$ & $0.00070 \pm 0.00004$ & $7.4 \pm 1.7$ \\
\hline
\end{tabular}

Table 3.4: Dose equivalent, absorbed dose, and quality factors measured at depth and angle in a concrete shield for $230 \mathrm{MeV}$ protons incident upon a $\mathrm{Pb}$ target. 


\section{Chapter 4}

\section{Transport Calculation Comparisons}

Computer techniques are frequently used in shielding design by modeling the geometry and source, and then using physical models to estimate radiation production and transport. Monte Carlo random walk techniques in which single interactions are modeled and discrete ordinate techniques in which analytical solutions to the Boltzmann transport equation are employed are often used. For proton accelerator shielding design, Monte Carlo techniques were used to estimate secondary neutron production and discrete ordinate techniques to evaluate neutron attenuation lengths $[8,54]$.

The results from most of these calculational attempts have not been compared with measurements due to a lack of data. In this chapter, our results are compared with calculations completed for the design of the Loma Linda Proton Radiotherapy Facility $[53,54]$, as well as with new Monte Carlo calculations. These comparisons provide a reference benchmark for the design of future facilities. 


\subsection{HETC-DO Calculations}

Alsmiller et al. combined Monte Carlo and discrete ordinate calculations to predict the attenuation properties of the neutrons produced by $200 \mathrm{MeV}$ proton bombardment of an Al target [8]. This method was used by Hagen et al. to predict the shielding required for the Loma Linda Medical Center Proton Therapy Facility $[53,54]$. This section discribes the results of these calculations, hereafter referred to HETC-DO, and compares them with our experimental results.

\subsubsection{Calculational Method}

Hagen et al. performed calculations for 150,200 and $250 \mathrm{MeV}$ protons incident upon stopping length $\mathrm{Fe}$ and $\mathrm{H}_{2} \mathrm{O}$ targets [54]. Neutron production was estimated using HETC [15]. Angular dependence was accounted for by considering neutron production in six angular bins, and used the output from each bin as a source for a separate ANISN [46] discrete ordinates run [54]. In each ANISN calculation, dose equivalent per incident proton is evaluated as a function of radius in a solid concrete sphere. Resulting dose equivalent values are presented as attenuation curves for each angular interval for 150,200 , and $250 \mathrm{MeV}$ protons incident upon $\mathrm{Fe}$ and $\mathrm{H}_{2} \mathrm{P}$ targets. HETC-DO dose equivalent values are not given for depths less than $100 \mathrm{~cm}$ since the production calculation neglected neutrons with energies below $20 \mathrm{MeV}$.

Table 4.1 lists the concrete composition used by Hagen. This composition contains far less hydrogen than typical concrete and the concrete used in our shielding measurements (Table 2.2). 


\begin{tabular}{|l|l|}
\hline Element & $\begin{array}{c}\text { Density } \\
\left(\frac{\text { atoms }}{\mathrm{cm}^{3}}\left(\times 10^{22}\right)\right)\end{array}$ \\
\hline Oxygen & 4.54 \\
Hydrogen & 0.386 \\
Silicon & 2.08 \\
Aluminum & 0.0482 \\
Iron & 0.107 \\
\hline
\end{tabular}

Table 4.1: Concrete composition used in the HETC-DO calculations, density 2.3 $\mathrm{g} \cdot \mathrm{cm}^{-3}[54]$.

\subsubsection{Parameterization of HETC-DO Results}

To compare our $230 \mathrm{MeV}$ results at $0^{\circ}, 22^{\circ}, 45^{\circ}$, and $90^{\circ}$ with HETC-DC calculations, HETC-DO results are parameterized by fitting to a simple analytic expression. In the angular range of $10^{\circ}$ to $120^{\circ}$, Freeman [49] fitted the HETC DO results at depths from 100 to $600 \mathrm{~cm}$ concrete to the form

$$
R^{2} H=A_{j}\left(\theta_{e f f}\right) 10^{B_{j}\left(\theta_{e f f}\right) R}
$$

for dose equivalent values at each energy $j$ and effective angle $\theta_{\text {eff }}$ relative to the incident proton beam direction. $A_{j}\left(\theta_{e f f}\right)$ and $B_{j}\left(\theta_{e f f}\right)$ are free parameters in the fit. Effective angles for the angular bin $\left(\theta_{i}, \theta_{f}\right)$ are determined using the method of Smith [98]

$$
\cos \theta_{e f f}=\frac{1}{2}\left(\cos \theta_{i}+\cos \theta_{f}\right)
$$

Freeman noted that parameters found in the fits, $A_{j}\left(\theta_{e f f}\right)$ and $B_{j}\left(\theta_{e f f}\right)$, are smooth functions of $\theta$ for a given energy $j$. Therefore, at each energy, production coefficients are fitted to the form

$$
A_{j}(\theta)=a_{j} 10^{k j}
$$


and slope coefficients are fitted to

$$
B_{j}(\theta)=b_{j}+c_{j} \theta+d_{j} \theta^{2}
$$

where the resulting coefficients are given in Table 4.2 .

\begin{tabular}{||l||c|c|c|c|c||}
\hline $\begin{array}{c}\text { Energy } \\
(\mathrm{MeV})\end{array}$ & $\begin{array}{c}a_{j} \\
\left(\mathrm{~cm}^{2} \cdot \mathrm{pSv} \cdot \mathrm{p}^{-1}\right)\end{array}$ & $\begin{array}{c}k_{j} \\
\left(\mathrm{dg}^{-1}\right)\end{array}$ & $\begin{array}{c}b_{j} \\
\left(\mathrm{~cm}^{-1}\right)\end{array}$ & $\begin{array}{c}c_{j} \\
\left(\mathrm{~cm}^{-1} \cdot \mathrm{dg}^{-1}\right)\end{array}$ & $\begin{array}{c}d_{j} \\
\left(\mathrm{~cm}^{-1} \cdot \mathrm{dg}^{-2}\right)\end{array}$ \\
\hline 150 & 171.1 & -0.0229 & -0.0104 & $-5.148 \cdot 10^{-5}$ & $2.155 \cdot 10^{-8}$ \\
200 & 292.1 & -0.0216 & -0.0091 & $-5.575 \cdot 10^{-5}$ & $9.974 \cdot 10^{-8}$ \\
250 & 428.3 & -0.0205 & -0.0086 & $-4.554 \cdot 10^{-5}$ & $4.978 \cdot 10^{-8}$
\end{tabular}

Table 4.2: Freeman's parameters for an angular fit to HETC-DO dose equivalent attenuation results for 150,200 , and $250 \mathrm{MeV}$ protons inciclent upon a stopping length Fe target. (Units: $\mathrm{p}=$ proton, $\mathrm{dg}=$ degrees) $[49]$.

For an easier comparisun to our data, these parameters are re-normalized to base exponential fits, i.e.

$$
R^{2} H=A_{j}^{\prime}\left(\theta_{e f f}\right) e^{B_{j}^{\prime}\left(\theta_{e f f}\right) R},
$$

with production coefficients

$$
A_{j}^{\prime}(\theta)=a_{j} e^{k_{j}^{\prime} \theta}
$$

and slope coefficients

$$
B_{j}^{\prime}(\theta)=b_{j}^{\prime}+c_{j}^{\prime} \theta+d_{j}^{\prime} \theta^{2}
$$

where the coefficients are now given in Table 4.3. Production and slope coefficients evaluated at each energy for angles of $0^{\circ}, 22^{\circ}, 45^{\circ}$, and $90^{\circ}$ are listed in Table 4.4. These values are fitted with a second order polynomial to allow interpolation to $230 \mathrm{MeV}$. Interpolated values are listed in 'Table 4.4. 
Errors in these coefficients are not computed since the values listed by Hagen [54] and the parameters computed by Freeman [49] are stated without error. Errors in the angular and energy interpolation are also unknown. A reasonable estimate of the errors is $\approx 10 \%$.

\begin{tabular}{|l|c|c|c|c|c|}
\hline $\begin{array}{l}\text { Energy } \\
(\mathrm{MeV})\end{array}$ & $\begin{array}{c}a_{j} \\
\left(\mathrm{~cm}^{2} \cdot \mathrm{Sv}_{\mathrm{p}}^{-1}\right)\end{array}$ & $\begin{array}{c}k_{j}^{\prime} \\
\left(\mathrm{dg}^{-1}\right)\end{array}$ & $\begin{array}{c}b_{j}^{\prime} \\
\left(\mathrm{cm}^{-1}\right)\end{array}$ & $\begin{array}{c}c_{j}^{\prime} \\
\left(\mathrm{cm}^{-1} \cdot \mathrm{dg}^{-1}\right)\end{array}$ & $\begin{array}{c}d_{j}^{\prime} \\
\left(\mathrm{cm}^{-1} \cdot \mathrm{dg}^{-2}\right)\end{array}$ \\
\hline 150 & 171.1 & -0.0527 & -0.0239 & $-1.185 \cdot 10^{-4}$ & $4.962 \cdot 10^{-8}$ \\
200 & 292.1 & -9.0497 & -0.0210 & $-1.284 \cdot 10^{-4}$ & $2.297 \cdot 10^{-7}$ \\
250 & 428.3 & -0.0472 & -0.0198 & $-1.049 \cdot 10^{-4}$ & $1.146 \cdot 10^{-7}$ \\
\hline
\end{tabular}

Table 4.3: Parameters for base $e$ exponential fit HETC-DO dose equivalent attenuation results for 150,200 , and $250 \mathrm{MeV}$ protons incident upon a stopping length $\mathrm{Fe}$ target. (Units: $\mathrm{p}=$ proton, $\mathrm{dg}=$ degrees).

\begin{tabular}{|c|c|c|c|c|}
\hline \multirow[b]{2}{*}{$\begin{array}{l}\text { Energy } \\
(\mathrm{MeV})\end{array}$} & \multicolumn{2}{|r|}{$0^{\circ}$} & \multicolumn{2}{|r|}{$22^{\circ}$} \\
\hline & $\begin{array}{l}\text { Slope } \\
(\mathrm{cm})\end{array}$ & $\begin{array}{c}\text { Intercept } \\
\left(\mathrm{cm}^{2} \cdot p S v \cdot p^{-1}\right)\end{array}$ & $\begin{array}{l}\text { Slope } \\
(\mathrm{cm})\end{array}$ & $\begin{array}{c}\text { Intercept } \\
\left(\mathrm{cm}^{2} \cdot \mathrm{pS}_{\mathrm{v}} \cdot \mathrm{p}^{-1}\right)\end{array}$ \\
\hline 150 & 41.8 & 171 & 37.7 & 54 \\
\hline 200 & 47.7 & 292 & 42.2 & 98 \\
\hline 230 & 49.9 & 372 & 44.3 & 129 \\
\hline \multirow[t]{2}{*}{250} & 50.5 & 428 & 45.4 & 152 \\
\hline & \multicolumn{2}{|r|}{$45^{\circ}$} & \multicolumn{2}{|r|}{$90^{\circ}$} \\
\hline 150 & 34.3 & 16.0 & 29.2 & 1.49 \\
\hline 200 & 38.1 & 31.2 & 32.7 & 3.32 \\
\hline 230 & 40.1 & 42.6 & 34.4 & 4.89 \\
\hline 250 & 41.2 & 51.2 & 35.3 & 6.12 \\
\hline
\end{tabular}

Table 4.4: Production and slope coefficients evaluated at $0^{\circ}, 22^{\circ}, 45^{\circ}$, and $90^{\circ}$ using parameterization of $\mathrm{HETC}-\mathrm{DO}$ results. Interpolated value at $2.30 \mathrm{MeV}$ is also listed. 


\subsubsection{Comparison with Measurements}

Calculated production and attenuation coefficients are now compared with the parameters found in fitting to the experimental data. Table 4.5 lists production coefficients for the $\mathrm{Fe}$ target data as well as for an average over the $\mathrm{Al}, \mathrm{Fe}$, and $\mathrm{Pb}$ targets.

\begin{tabular}{||l|c|c|c||}
\hline Angle & $\begin{array}{c}\text { Experimental } \\
\text { Fe Target } \\
\left(\mathrm{pSv} \cdot \mathrm{cm}^{2} \cdot \mathrm{p}^{-1}\right)\end{array}$ & $\begin{array}{c}\text { Experimental } \\
\text { Target Average } \\
\left(\mathrm{pSv} \cdot \mathrm{cm}^{2} \cdot \mathrm{p}^{-1}\right)\end{array}$ & $\begin{array}{c}\text { HETC-DO } \\
\text { Fe Target } \\
\left(\mathrm{pSv} \cdot \mathrm{cm}^{2} \cdot \mathrm{p}^{-1}\right)\end{array}$ \\
\hline $0^{\circ}$ & $64 \pm 7$ & $66 \pm 5$ & 372 \\
$22^{\circ}$ & $34 \pm 3$ & $35.7 \pm 2.3$ & 129 \\
$45^{\circ}$ & $13.4 \pm 1.2$ & $15.3 \pm 0.9$ & 42.6 \\
$90^{\circ}$ & $4.08 \pm 1.0$ & $4.43 \pm 0.74$ & 4.89 \\
\hline
\end{tabular}

Table 4.5: Measured normalization constants and comparison with values predicted using fit to $\mathrm{HETC}-\mathrm{DO}$ results.

For our results, minimal differences exist between the Fe data and the $\mathrm{Al}$ and $\mathrm{Pb}$ data, hence, the target average data are very close to the Fe data. The HETCDO calculations also yielded small deviations between $\mathrm{Fe}$ and $\mathrm{H}_{2} \mathrm{O}$ values $[53,54]$.

Substantial differences exist between measured data and HETC-DO calculated results. HETC-DO overestimates the production coefficient at $0^{\circ}, 22^{\circ}$, and $45^{\circ}$. This effect is most pronounced at $0^{\circ}$ where HETC-DO overestimates the yield by a factor of six. At $22^{\circ}$, the yield is overestimated by 3.6 times whereas at $90^{\circ}$, yield values agree within error.

The overestimation by HETC at small angles is consistent with nentron spectrum measurements $[22,28,29,35,48,79,10 \pi]$. In particular, Wachter $\mid 107]$ found that for $160 \mathrm{MeV}$ protons incident upon a stopping length $\mathrm{Al}$ target, the neu- 
tron production calculated using HETC at $10^{\circ}$ over-predicts his measurements by a factor of 3.5. A comparison by Meier et al. $[73,74,75]$ indicates that HETC overestimates the high energy neutron yield at $7.5^{\circ}$ for $113 \mathrm{MeV}$ protons by a factor of two, but the agreement is good for $256 \mathrm{MeV}$ proton bombardment. (Figures 1.1 and 1.2). Deviations among various HETC calculations are most likely due to the use of different parameters and cross section libraries.

The difference in the composition of the concrete used in the experiment and HETC-DO calculation could also contribute to the overestimation by HETC-DO. Scattering of neutrons on hydrogen is an effective means of degrading neutron energy [83]. However, such scattering only plays an iraportant role where the cross section dominates, i.e. below a few MeV [83].

For reactor shielding applications, a decrease in the water content by $1 / 3$, results in an increase of the neutron leakage by a factor of three, as well as an increase in the thermal flux by a factor of five [56]. Due to this, it is recommended that the minimum fixed water content not be less than $5 \%$ by weight. for ordinary concrete used in shielding applications [56]. Based upon the composition supplied with the $\mathrm{HETC}-\mathrm{DO}$ calculations, their $\mathrm{H}_{2} \mathrm{O}$ content was $2.5 \%$ by weight compared with our $15 \%$. Ordinary concrete usually contains between $5-15 \%$ water $[56,83]$.

The equilibrium neutron spectrum present in the shield used in the HETC:DO calculations is expected to differ from that in the shield used in these measurements due to these composition variances. Since the HETC-DO shield contains less hydrogen, more neutrons below $10 \mathrm{MeV}$ are expected to exist in that 
shield $[56,83]$. Further calculations which provide the neutron spectrum at depth within the shield are necessary to quantify this effect.

The attenuation lengths of neutron shielding materials are determined principally by the high energy neutrons $[83,105]$, provided mechanisms exist by which low energy neutrons are removed from the spectrum. Above $20 \mathrm{MeV}$, inelastic interactions dominate over elastic scattering in neutron attenuation [8.3]. Therefore, bulk shield density is of primary importance, and hydrogen content only determines the shape of the low energy equilibrium spectrum.

Experimentally determined mass attenuation lengths are compared with the results from HETC-DO calculations in Table 4.6. The deviation observed is quite large, and is likely caused by differences in the shield compositions used. Figure 4.1 shows a plot of the over-prediction HETC-DO calculation as a function of depth at each angle.

\begin{tabular}{|cc|c|}
\hline Angle & $\begin{array}{c}\text { Experimental } \\
\text { Data } \\
\left(\mathrm{g} \cdot \mathrm{cm}^{-2}\right)\end{array}$ & $\begin{array}{c}\text { HETC-DO } \\
\text { Results } \\
\left(\mathrm{g} \cdot \mathrm{cm}^{-2}\right)\end{array}$ \\
\hline $0^{\circ}$ & $89 \pm 3$ & 115 \\
$22^{\circ}$ & $85 \pm 3$ & 102 \\
$45^{\circ}$ & $77.8 \pm 2.4$ & 92.1 \\
$90^{\circ}$ & $58 \pm 4$ & 79.0 \\
\hline
\end{tabular}

Table 4.6: Comparison of mass attenuation lengths measured and calculated using HETC-DO.

Due to the differences in the concrete composition, comparisons are also clone on a thickness basis. Table 4.7 shows the results of this comparison. The agreement between the attenuation lengths determined is good. Note that material 


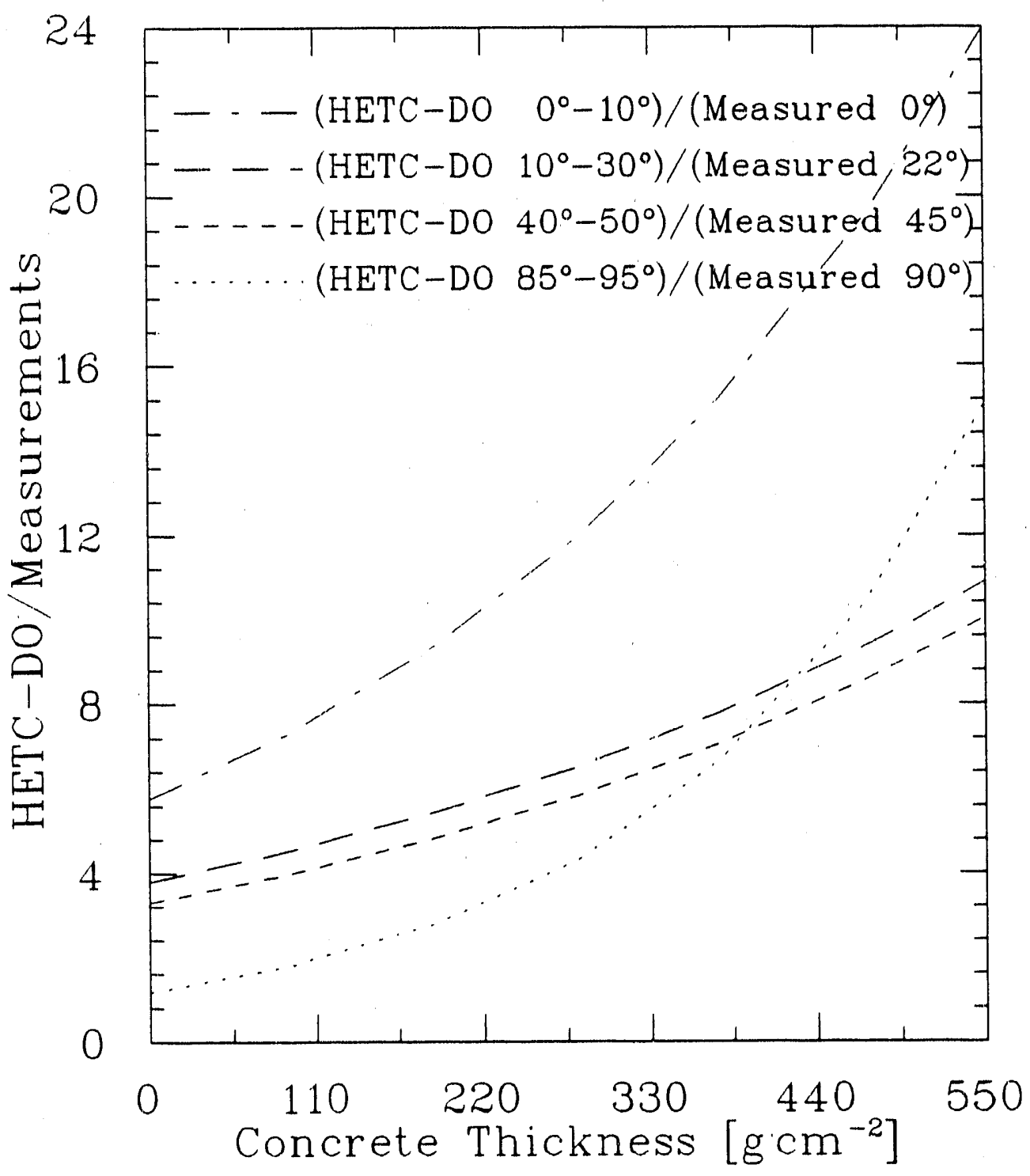

Figure 4.1: Ratio of HETC-DO predicted dose equivalent values to measured dose equivalent values at the same mass thickness into the shield versus shield depth. 
density and composition differences have not been accounted for. The agreement is believed to be fortuitous, rather than to represent a valid comparison. The ratio of the computed to measured values is plotted in Figure 4.2 as a function of depth at each angle. A suggested future calculation to estimate material effects is re-computation of the ANISN part of the HETC-DO calculation using the concrete composition used in the measurements.

\begin{tabular}{|c|c|c|}
\hline Angle & $\begin{array}{c}\text { Experimental } \\
\text { Data } \\
(\mathrm{cm})\end{array}$ & $\begin{array}{c}\text { HETC-DO } \\
\text { Results } \\
(\mathrm{cm})\end{array}$ \\
\hdashline $0^{\circ}$ & $47.1 \pm 1.5$ & 49.9 \\
$22^{\circ}$ & $45.4 \pm 1.6$ & 44.3 \\
$45^{\circ}$ & $41.3 \pm 1.3$ & 40.1 \\
$90^{\circ}$ & $30.8 \pm 2.2$ & 34.4 \\
\hline
\end{tabular}

Table 4.7: Comparison of experimental and HETC-DO determined attenuation lengths, $\lambda$.

\subsection{Analytical Methods}

Several authors provide analytical methods for estimating the shielding required for proton accelerators. The dose equivalent attenuation characteristics for monoenergetic neutrons with energies between 50 and $350 \mathrm{MeV}$ were calculated by Braid [33]. To estimate the shielding required for proton accelerators, Braid [33] used analytic functional fits provided by Alsmiller |1| to Bertini's intranuclear cascade data $[25]$ and inelastic interaction probabilities from Janni $[67]$ to determine thick target neutron production. By integrating the monoenergetic at- 


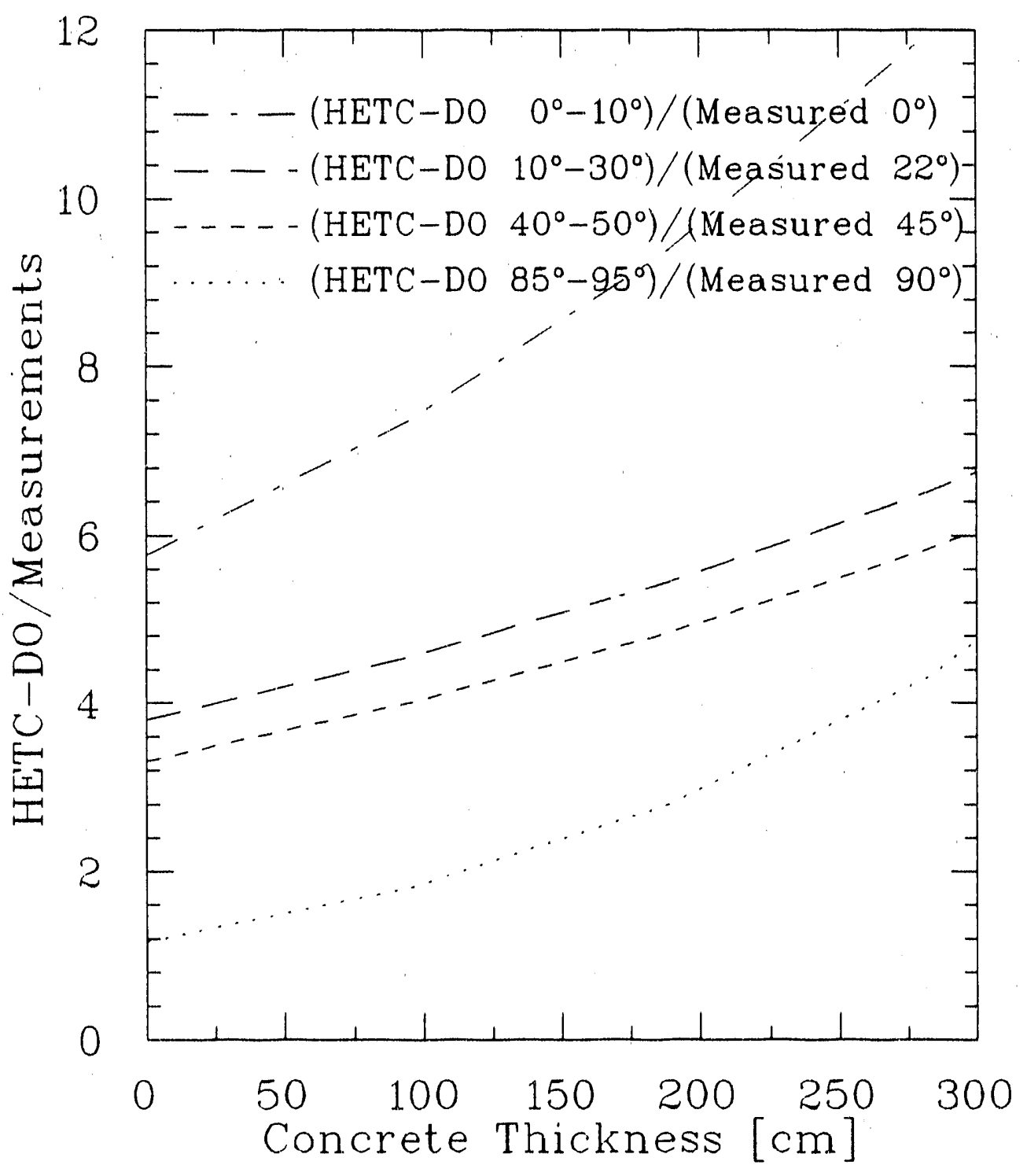

Figure 4.2: Ratio of HETC-DO predicted dose equivalent values to measured dose equivalent values evaluated at the same shield depth $(\mathrm{cm})$ versus shield depth. 


\begin{tabular}{|l|c|c|c|c||} 
& $\begin{array}{c}\lambda \\
(\mathrm{cm})\end{array}$ & $\begin{array}{c}\mathrm{P} \\
\mathrm{pSv} \cdot \mathrm{cm}^{2} \cdot \text { proton }^{-1}\end{array}$ & $\begin{array}{c}\lambda_{c} \\
\lambda_{m}\end{array}$ & $\begin{array}{c}P_{c} \\
P_{m}\end{array}$ \\
\hline Measured $\left(0^{\circ}\right)$ & $47.1 \pm 1.5$ & $64 \pm 7$ & - & - \\
IAEA-Braid $\left(0^{\circ}-30^{\circ}\right)$ & 49.2 & 87.4 & 1.05 & 1.37 \\
& & & & \\
Measured (90 & $30.8 \pm 2.2$ & $4.1 \pm 1.0$ & - & - \\
IAEA ("Lateral") & 38.9 & 15.8 & 1.26 & 3.88 \\
Tesch ("Lateral") & 41.1 & 4.5 & 1.34 & 1.10 \\
\hline
\end{tabular}

Table 4.8: Comparison of measured attenuation parameters with those using the method of Braid [33,57], and with the method of Tesch [105].

tenuation data over the calculated neutron spectrum, dose rates outside a given shielding thickness were determined.

Using this procedure, the IAEA $[57]$ published recommended attenuation coefficients for concrete at $90^{\circ}$ and in the forward direction $\left(0^{\circ}-30^{\circ}\right)$ for the neutrons produced by 50 to $400 \mathrm{MeV}$ protons incident upon thin and thick Cu targets. Table 4.8 compares these values, interpolated to $230 \mathrm{MeV}$, with our measurements. In the forward direction, the attenuation parameters agree with our measured values at $0^{\circ}$. Values at $90^{\circ}$, however, are overestimated.

Tesch [105] compiled lateral shielding data and calculations for 50 to $1000 \mathrm{MeV}$ proton accelerators and estimated values of attenuation lengths and production terms in this energy region. These values are also compared with our measurements in Table 4.8. The production term is predicted accurately and the attenuation term is overestimated. 


\subsection{LAHET Code}

Due to the material differences between our measurements and those modeled in the HETC-DO calculations and to the recent improvements in the nuclear models in a recent revision HETC, further Monte Carlo calculations using the Los Alamos revision of HETC, LAHET, were undertaken. In this section, LAHET' is briefly described as implemented for comparison with our experiment. Comparisons of calculated and measured values are presented at the end of this section.

\subsubsection{LAHET, A Brief Overview}

In recent years, Los Alamos National Laboratory (LANL) put forth a major effort in the revision of the High Energy Transport Code (HETC) originally developed at Oak Ridge National Laboratory. Many new features were added, therefore the code was renamed LAHET [8T|. LAHET is the principal code of the LAHET Code System (LCS) which was developed for analysis of both high and low energy neutron and photon interactions in matter.

LCS consists of the computer programs LAHET, HMCNP, PHT, XSEC, and HTAPE. LAHET treats all interactions of heavy charged particles, but only deals with neutrons above a $20 \mathrm{MeV}$ particle cutoff energy. 'To allow the inclusion of effects produced by neutrons below $20 \mathrm{MeV}$, LAHET records these events in a file which can be used as a source file for subsequent HM('NP calculations. HWinP is a minor revision the low energy Nonte Carlo nentron and photon transport code MCINP [34] which accepts sonrce files written by LAHET, as well 
as other distributed source files. The PHT code is used to construct gammaray source files from collision information recorded on LAHET history files for use in $\mathrm{HMCNP}$ calculations [87]. XSEC calculates double-differential particle production cross sections for primary beam interactions from LAHET history files. The HTAPE code analyzes history files written by LAHET and HMCNP to determine surface currents, surface fluxes, particle production, energy deposition, excitation energies, and other interaction products. HTAPE can also be used with response functions to modify any tallied history.

The major differences between LAHET and HETC include the Fermi breakup model replacing the evaporation model for breakup of light nuclei, the addition of a multistage preequilibrium excitation model as an intermediate stage between the intranuclear cascade and evaporation stages of the nuclear interaction, and the addition of a few alternative nuclear level density parameterizations. LAHET defaults to using the Bertini [29] intranuclear cascade model. An alternative intranuclear cascade model, ISABEL $[109,110]$, is included amongst LAHET's options. Since LAHET is coupled to HMCNP thro:igh LCS, the geometrical setup of MCNP has been implemented for user convenience.

\subsubsection{Previous LAHET results}

Comparisons of LAHET calculations with neutron yield measurements are useful to understanding how well LAHET can be expected to agree with production and attenuation measurements. Recently, benchmark comparisons between measured thick target neutron yield and LAHET calculations were completed at Los 
Alamos [88]. Neutron yield was measured and calculated for 113 and $256 \mathrm{MeV}$ protons incident upon a variety of stopping length targets at a few angles.

Figure 4.3 shows plots of the measured and calculated values using LAHET with the preequilibrium model between the cascade an evaporative stages for 113 $\mathrm{MeV}$ protons bcmbarding a stopping-length Fe target [74]. The ratio between calculated and measured values is plotted in Figure 4.4. LAHET predicts neutron production quite well. However at $7.5^{\circ}$, the neutron yield of the highest energy components is slightly overestimated. At $150^{\circ}$, the agreement is far better than values calculated using HETC (Figure 1.1).

Measured and LAHET calculated neutron yields for $256 \mathrm{MeV}$ protons incident upon a stopping length Fe target are plotted in Figure 4.5 [75]. The ratios between calculated and measured values are shown in Figure 4.6. Again, the inclusion of the preequlibrium model in LAHET calculations improves agreement with measured values, especially at large angles. (Figure 4.7).

The ability of LAHET to predict dose equivalent values and attenuation lengths is limited by the estimation of the yield. Where LAHET overestimates neutron yield, particularly in the high energy region, the dose equivalent values and the attenuation lengths will be overestimated if the transport processes mimic reality.

\subsubsection{Calculation Method}

Our calculations included running LAHET with the majority of the parameters in their default settings, that is, the Bertini intranuclear cascacle model |29|, the 


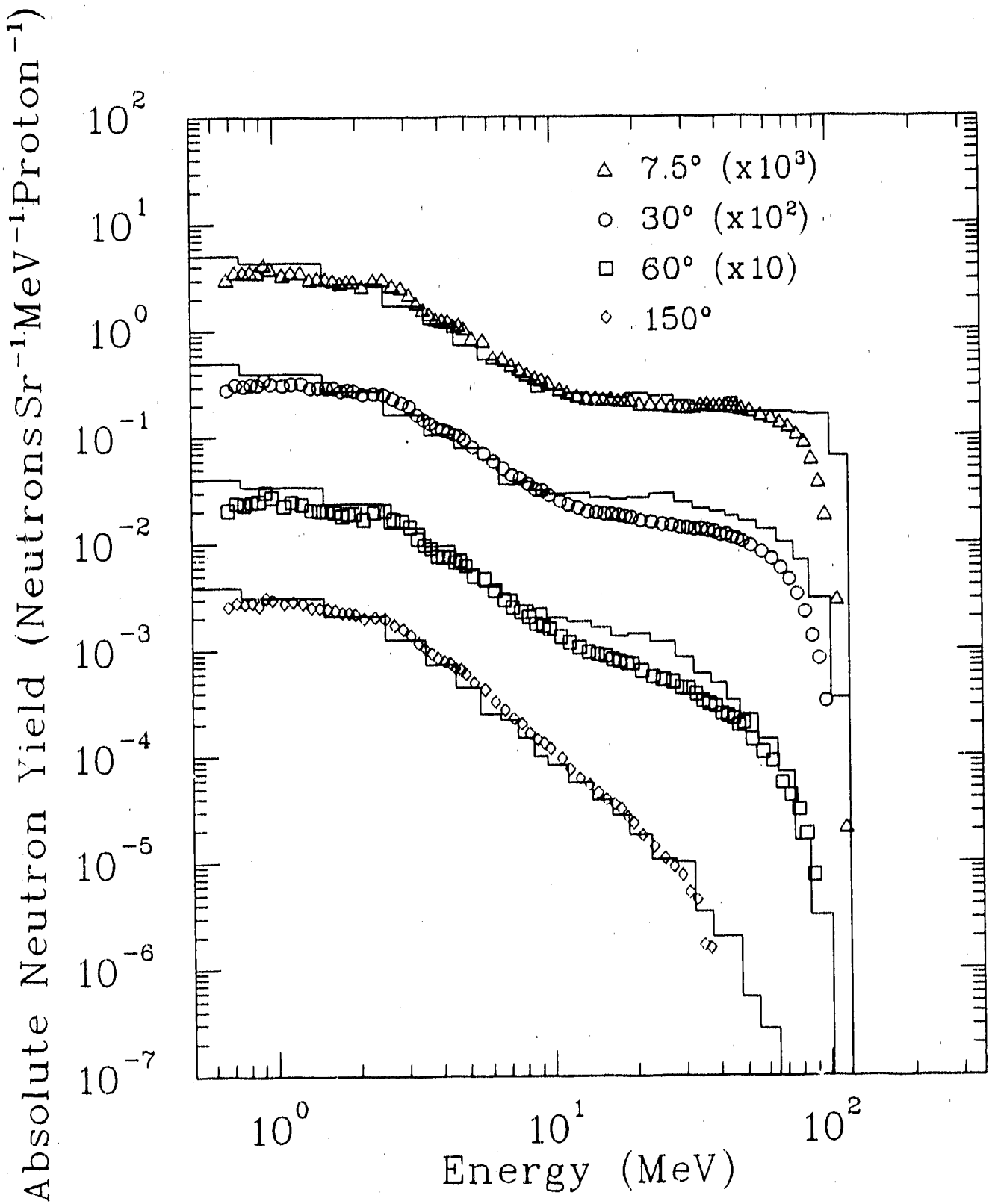

Figure 4.3: Neutron yield at $7.5^{\circ}, 30^{\circ}, 60^{\circ}$ and $150^{\circ}$ for $11.3 \mathrm{MeV}$ protons incident upon stopping length Fe targets. Data points measured by Meier $|74|$ and calculated values (histogram) from LAHET by Prael [88]. 


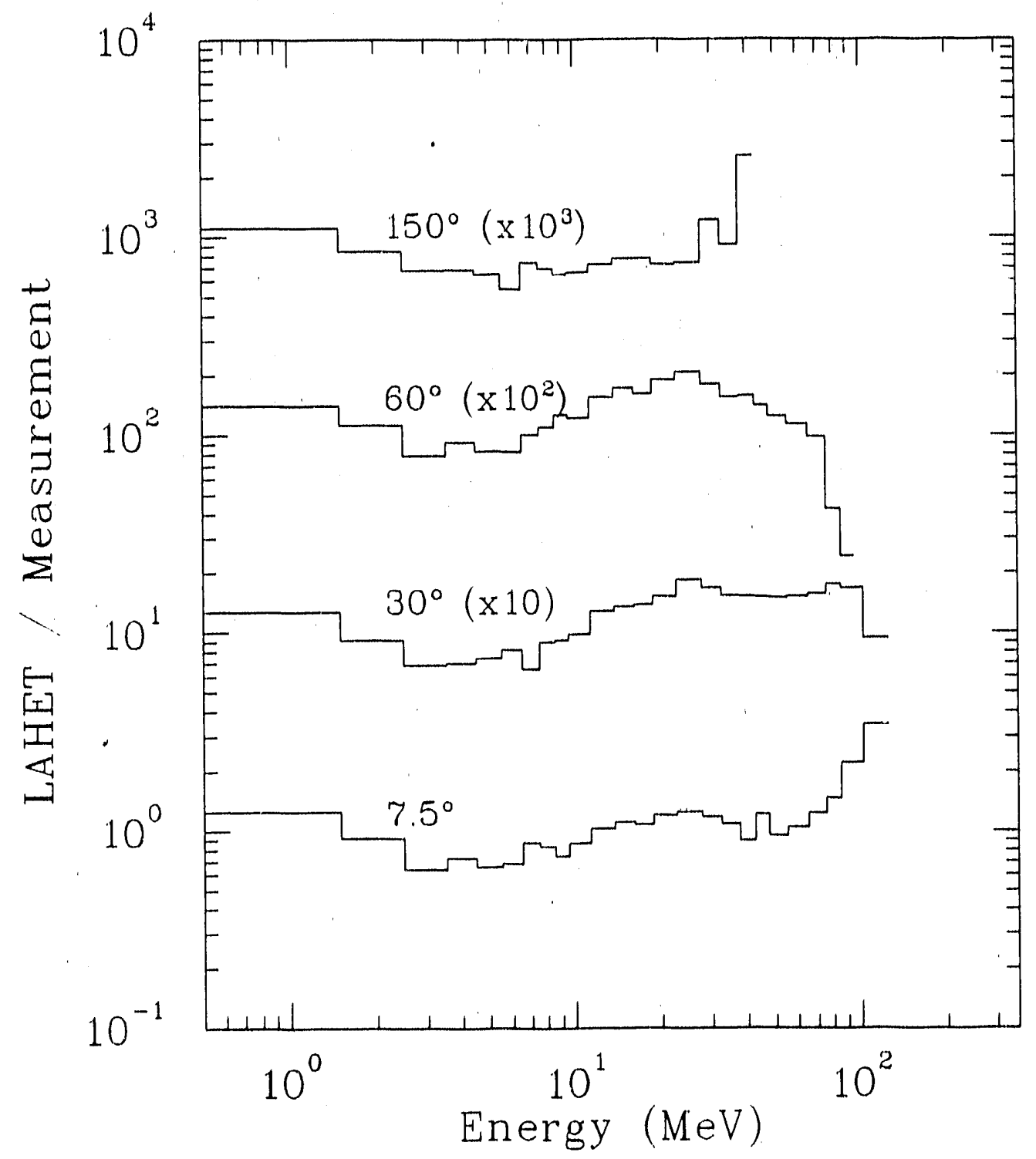

Figure 4.4: Ratio of calculated and measured neutron yield at $7.5^{\circ}, 30^{\circ}, 60^{\circ}$ and $150^{\circ}$. Measured by Meier [74] and calculated using LAHET by Prael [88]. 

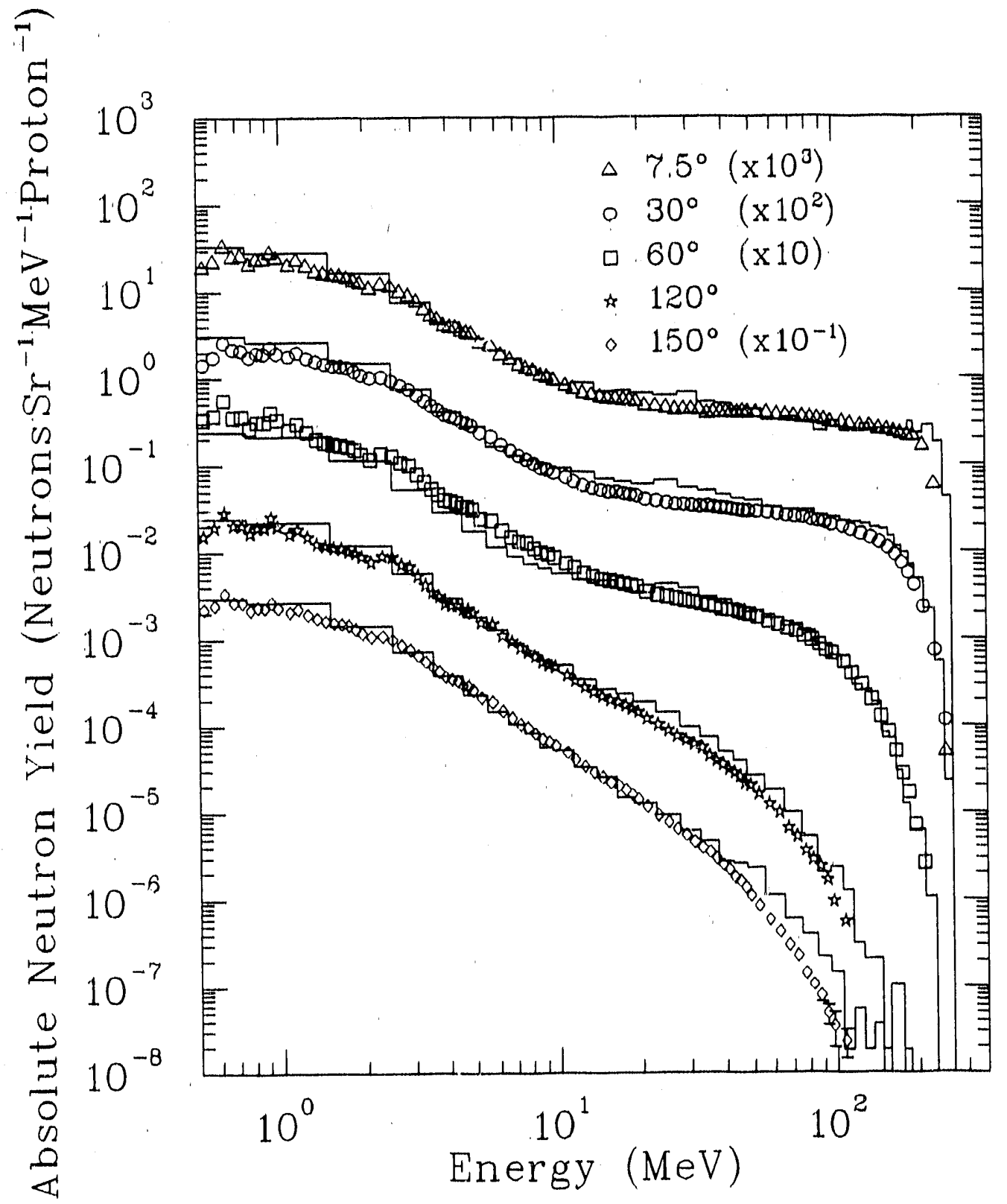

Figure 4.5: Neutron yield at $7.5^{\circ}, 30^{\circ}, 60^{\circ}, 120^{\circ}$ and $150^{\circ}$ for $256 \mathrm{MeV}$ protons incident upon stopping length Fe targets measured by Meier $|75|$ and calculated using LCS by Prael [88]. C'alculated values are shown as histograms. 


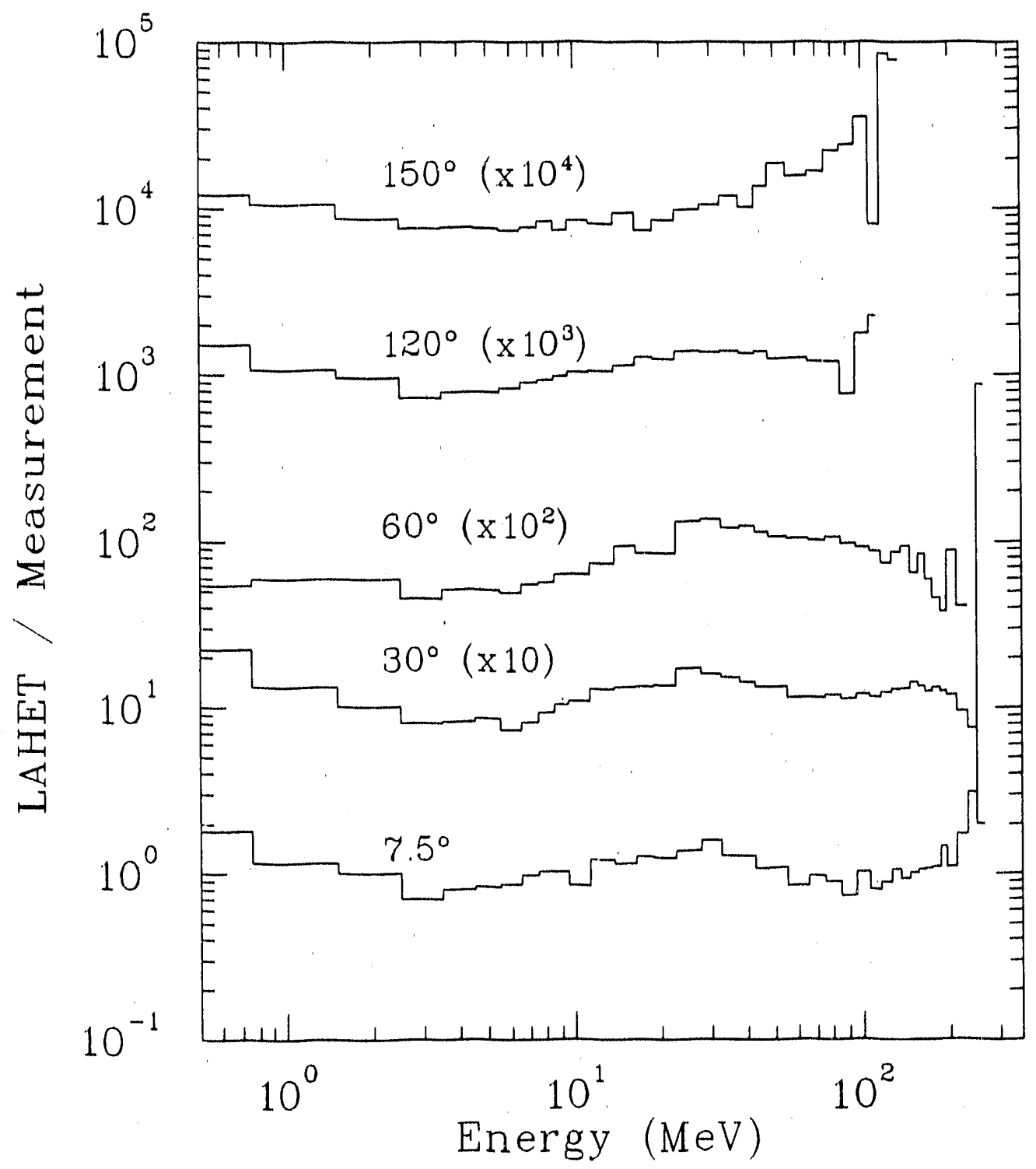

Figure 4.6: Ratio of calculated and measured neutron yield at $7.5^{\circ}, 30^{\circ}, 60^{\circ}$ and $150^{\circ}$. Measured by Meier [75] and calculated using LCSS by Prael [88]. 


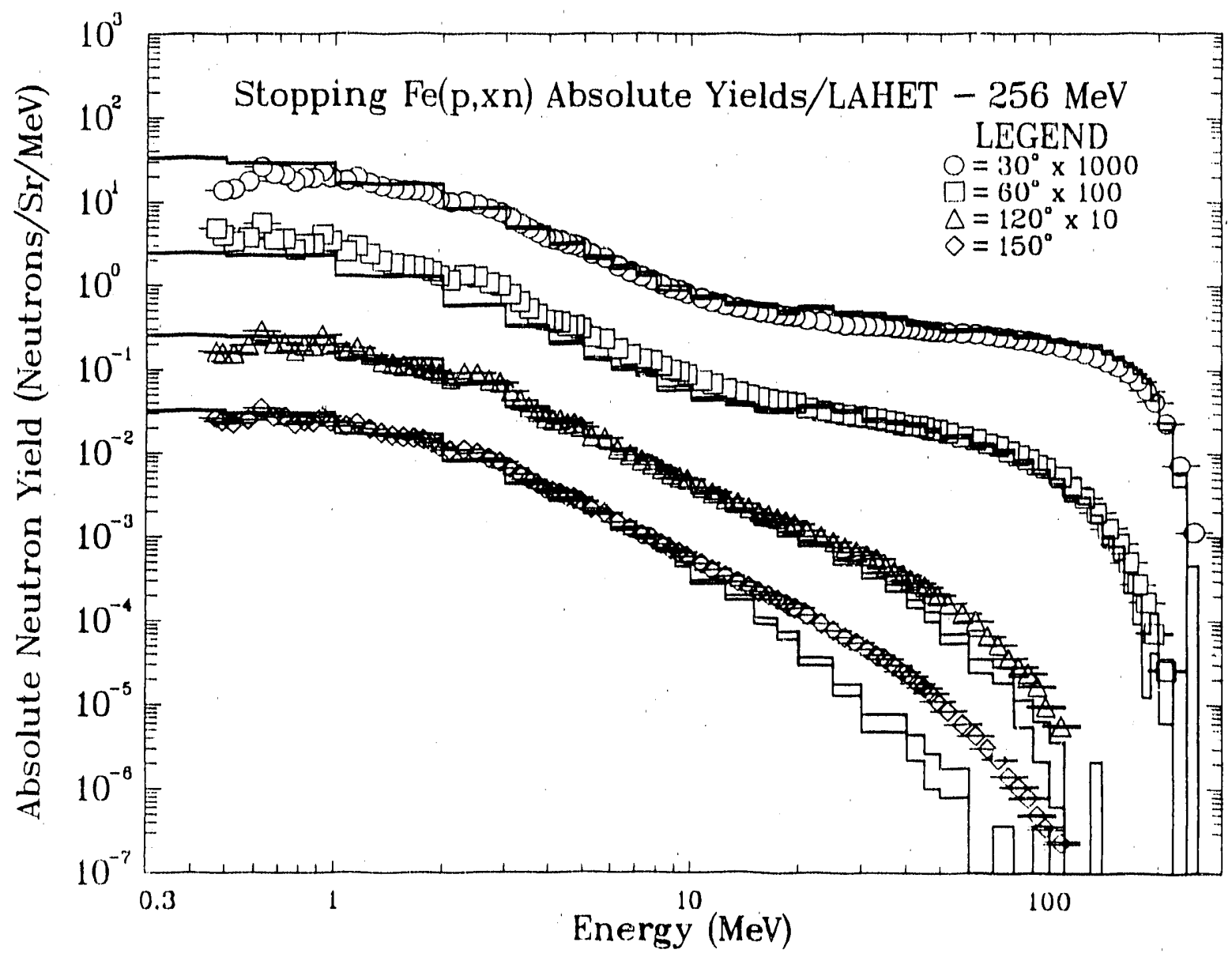

Figure 4.7: Neutron spectra produced by $256 \mathrm{MeV}$ protons incident upon $\mathrm{Fe}$ and LAHET calculated yield without inclusion of the preequilibrium model. Agreement at large angles is much poorer than when preequilibrium neutrons are included (Figure 4.5.) This figure was reproduced from Prael [88]. 
Gilbert-Cameron-Cook-Ignatyuk level density model [86], and the Fermi breakup model. The only crucial non-default parameter is the use of the preequilibrium model following the intranuclear cascade. The preequilibrium model is employed since it improves agreement with the neutron spectrum measurements at large angles [88].

Differences between our LCS calculations and previous calculations $[8,54]$ are the inclusion of the preequilibrium model following the intranuclear cascade and in the use of Monte Carlo techniques for transport of neutrons and photons into the shielding.

Hagen et al. [54], used HETC to obtain the target neutron spectrum. Hence, they only included neutrons above $20 \mathrm{MeV}$. Here, the target neutron and photon production is calculated using LCS with inclusion of the preequilibrium model. The coupling of LAHET with HMCNP allowed our work to include photons and neutrons down to thermal energies.

For transport of the neutrons into the shield, Hagen used ANISN [46], a discrete ordinates code, for which the neutron spectrum in a given angular bin is used as an isotropic point source in the center of a concrete sphere. In discrete ordinates codes, the solution tor the Boltzrnann transport equation is approximated using discrete variables in a number of energy bins. The neutron thence multiplied by ICRU 20 [60] recommended fluence-to-dose conversion factors are then used to calculate dose equivalent as a function of radius in the sphere. For our calculations, LAHET and HMCNP are used to transport neutrons and photons into a shielding geometry very similar to that used in our experiment. 
In our LAHET calculations, neutron fluence, absorbed dose, and dose equivalent are evaluated at multiple depths and angles in the shield using HTAPE to score the neutron flux, and using the HTAPE response function option to determine absorbed dose and dose equivalent. Response functions are based the upon values compiled by Belogorlov et al. [23]. Belogorlov tabulates and plots response as a function of neutron energy and depth in a tissue-equivalent slab. Following the method of the ICRP 51 [59], the maximum response independent of depth is used for determination of absorbed dose and dose equivalent values from the neutron spectrum. This procedure may result in overestimation of dose values for some neutron energies.

Figure 4.8 shows a plot of the dose equivalent response function as well as data recommended by the IC:RP [59]. These values are in excellent agreement since they are derived from the same set of calculations. Note that these conversion coefficients refer to the dose equivalent for neutrons as defined prior to 1985. The ICRP now recommends that these values be multiplied by a factor of 2 to obtain the dose equivalent as redefined for neutrons in $1985[58,59]$. This factor was not included in the present calculations. The absorbed dose response function used is plotted in Figures 4.9 .

\subsubsection{Geometry}

The geometry used in the LCS calculation is a simplified version of the geometry used in the measurements (Figure 4.10). The target chosen is idlentical to the Fe target used in the experiment, length $7.51 \mathrm{~cm}$, diameter $11.6 \mathrm{~cm}$. Located 


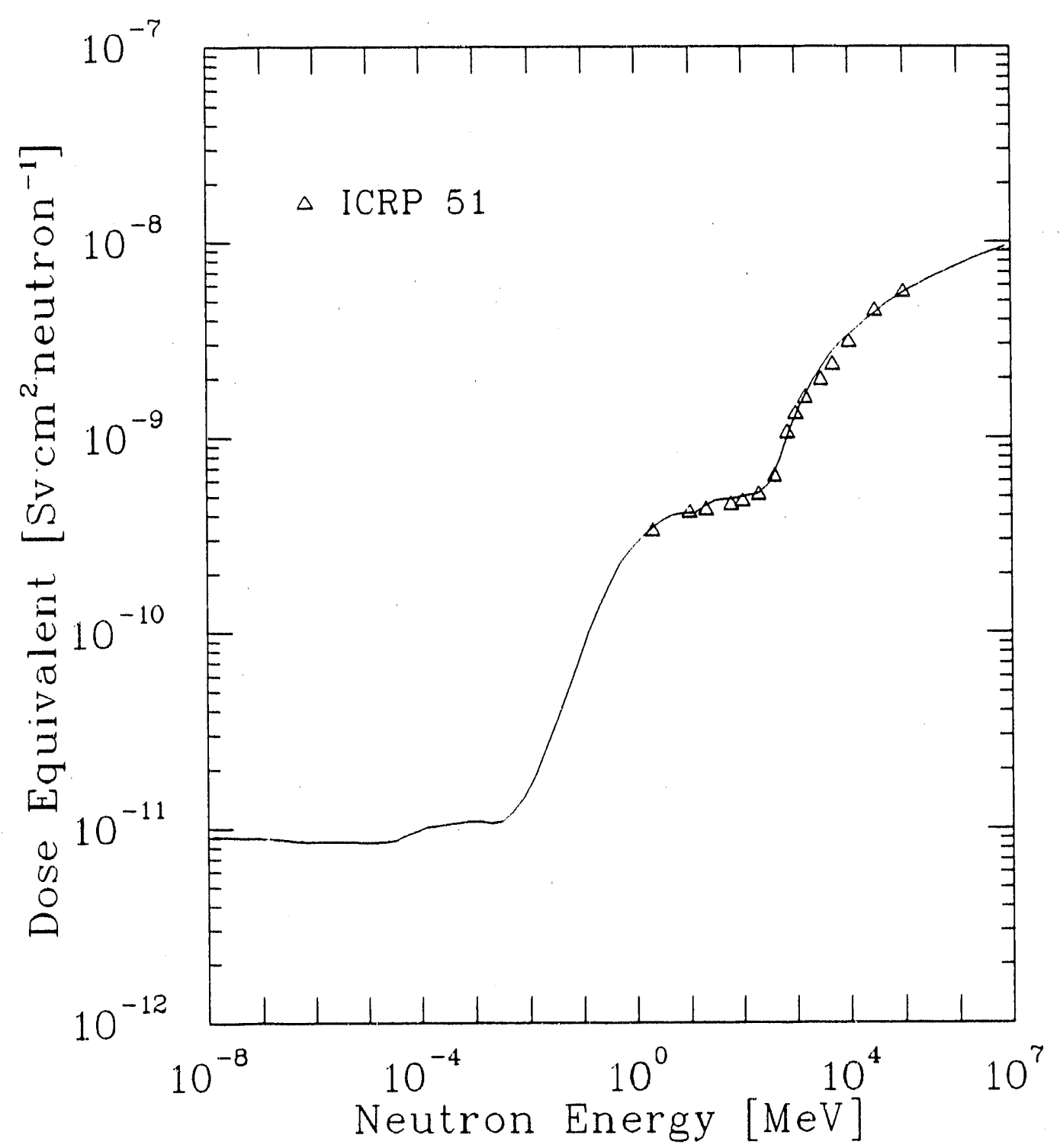

Figure 4.8: Neutron fluence to dose equivalent conversion factors used in these calculations. Values taken from Belogorlov $[23$ | and ICRP recommendations [59]. Note, ICRP recommendations refer to the definition of the quality factor for neutrons before 1985. The IC'RP recommends that the quality factor for neutrons be doubled $[59]$. 


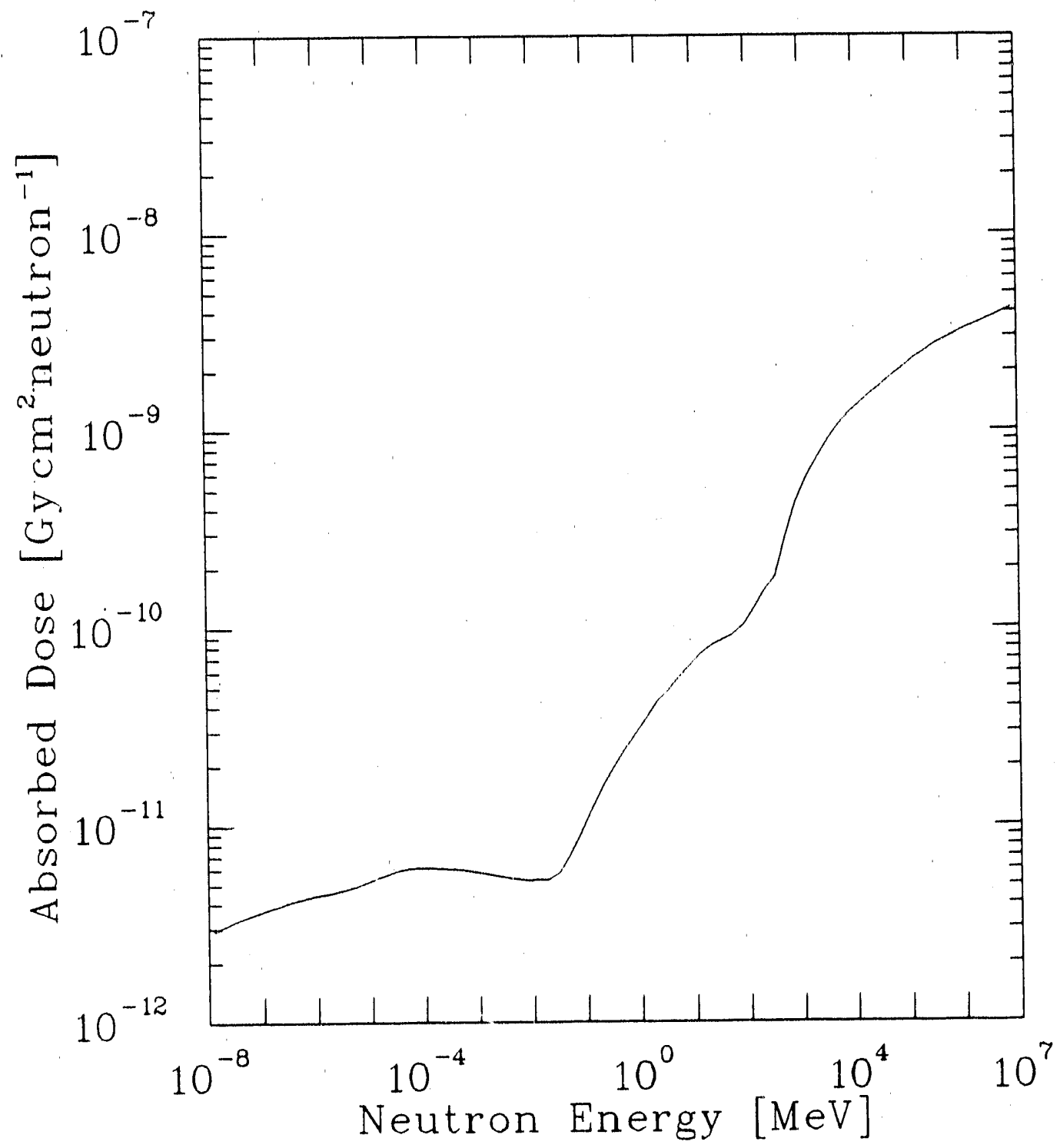

Figure 4.9: Neutron fluence to absorbed dose conversion factors used in these calculations as suggested by Belogorlov [23]. 
at the target effective neutron production center, calculated in Section 2.4, is a one meter diameter air sphere, followed by a four meter thick concrete sphere. The concrete composition used is identical to that used in our experiment (Table 2.2). To allow scoring of particle crossings and determination of dose at multiple depths, the concrete sphere is divided into a series of imaginary concentric spheres. Table 4.9 lists the radii and concrete depths of these imaginary spheres.

Calculations are performed with two different angular binnings. Large angular intervals are used to provide estimates of the neutron and photon spectrum as well as absorbed dose and dose equivalent values for determination of the mean radiation quality. The angular intervals, listed in Table 4.10, are chosen so the effective angles, computed using Equation 4.2, are ecrual to angles used in our measurements. Smaller angular bins, listed in Table 4.11 , are used for a restricted set of calculations to study the angular dependence of the dose equivalent attenuation.

\subsubsection{Results}

Output from the LCS and HMCNP calculations are edited to determine the neutron and photon fluence crossing each sphere at depth within the shield as well as absorbed dose and dose equivalent using HTAPE.

Figures 4.11 shows a plot of the LCS calculated neutron fluence in several angular bins crossing the air-shield interface. The values computed at low energies are shown in Figure 4.12. The photon fluence values crossing the air to 


\section{Eग Concrete}

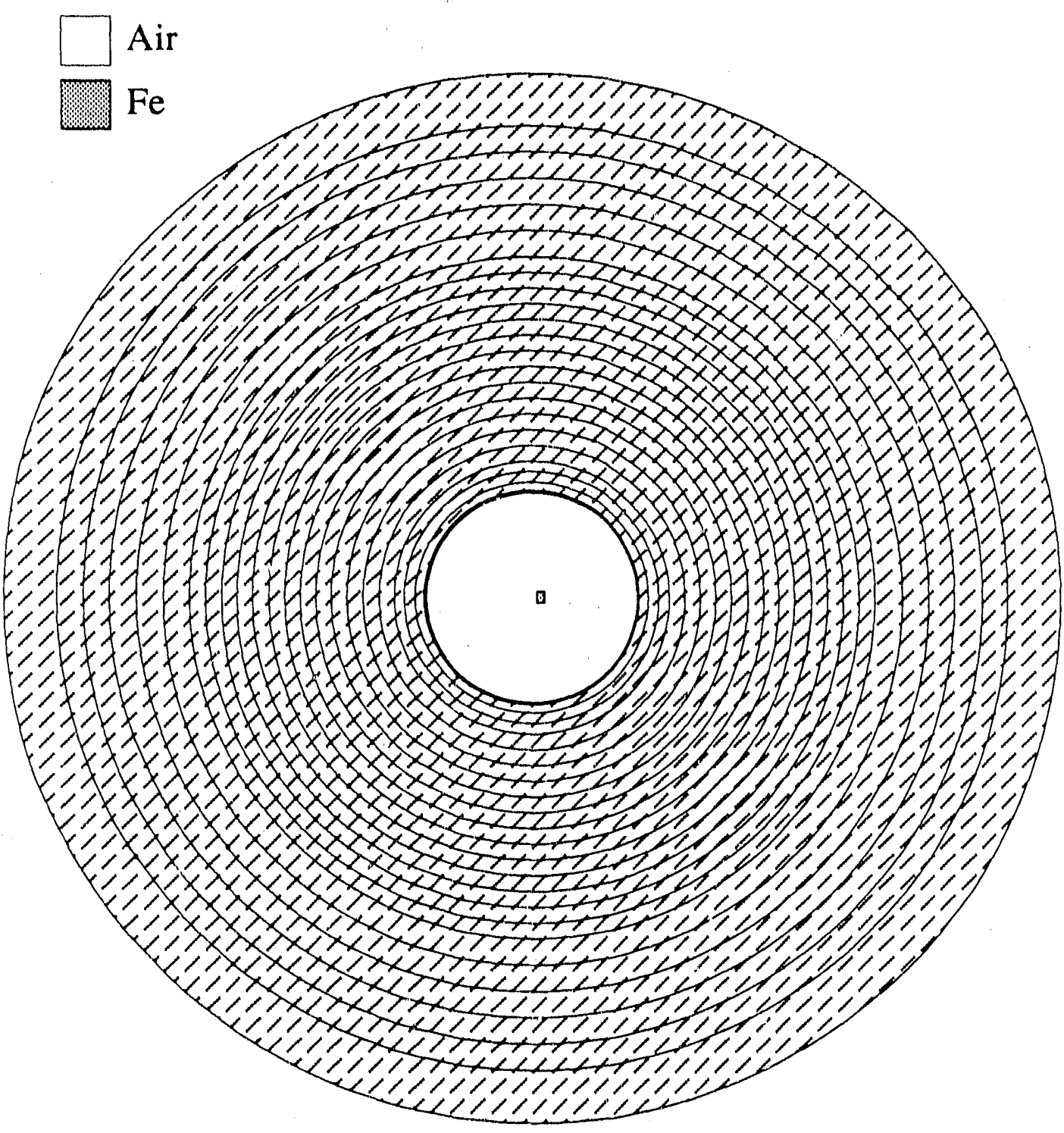

Figure 4.10: Geometry used in LCS calculation. In center is an iron target which is surrounded by one meter of air, followed by concentric concrete spheres at depths listed in Table 4.9. 


\begin{tabular}{||l|c|}
\hline Distance to & Concrete \\
Target & Thickness \\
$(\mathrm{cm})$ & $(\mathrm{cm})$ \\
100 & 0 \\
102 & 2 \\
105 & 5 \\
110 & 10 \\
120 & 20 \\
130 & 30 \\
145 & 45 \\
160 & 60 \\
175 & 75 \\
190 & 90 \\
205 & 105 \\
220 & 120 \\
235 & 135 \\
250 & 150 \\
265 & 165 \\
280 & 180 \\
295 & 195 \\
310 & 210 \\
325 & 225 \\
350 & 250 \\
375 & 275 \\
400 & 300 \\
425 & 325 \\
450 & 350 \\
500 & 400 \\
\hline &
\end{tabular}

Table 4.9: Depths for scoring events in concrete sphere for LCS calculations. 


\begin{tabular}{||l|c||}
\hline $\begin{array}{l}\text { Angular } \\
\text { Interval }\end{array}$ & $\begin{array}{c}\text { Effective } \\
\text { Angle }\end{array}$ \\
\hline $0^{\circ}-10^{\circ}$ & $7.5^{\circ}$ \\
$10^{\circ}-30^{\circ}$ & $22^{\circ}$ \\
$30^{\circ}-60^{\circ}$ & $45^{\circ}$ \\
$60^{\circ}-120^{\circ}$ & $90^{\circ}$ \\
$120^{\circ}-180^{\circ}$ & $139^{\circ}$ \\
\hline
\end{tabular}

Table 4.10: Angular binning used in LCS calculations for determination of neutron and photon fluence, absorbed dose, and dose equivalent.

\begin{tabular}{||l||}
\hline Angular \\
Interval \\
\hline $0^{\circ}-30^{\circ}$ \\
$30^{\circ}-40^{\circ}$ \\
$40^{\circ}-50^{\circ}$ \\
$50^{\circ}-60^{\circ}$ \\
$60^{\circ}-75^{\circ}$ \\
$75^{\circ}-85^{\circ}$ \\
$85^{\circ}-95^{\circ}$ \\
$95^{\circ}-105^{\circ}$ \\
$105^{\circ}-120^{\circ}$ \\
$120^{\circ}-180^{\circ}$ \\
\hline
\end{tabular}

Table 4.11: Angular binning used in LCS calculations for determining dose equivalent at depth in the shield. 
shield interface are plotted in Figure 4.13.

Of a greater interest is the neutron and photon spectrum at depth in the shield, since these spectra contribute to the dose at depth. The calculated neutron spectra in several angular bins at $105 \mathrm{~cm}$ concrete are plotted in Figures 4.14 and 4.15. The photon fluence spectra are plotted in Figure 4.16.

Calculated dose equivalent values at depth in the concrete in each angular interval are shown in Figure 4.17. The rapid attenuation of the low energy neutron component is quite apparent, as well as the decrease in attenuation length with increasi.ıg angle. Tables 4.15-4.19 list the calculated values of absorbed dose, dose equivalent, and mean radiation qualities. Mean radiation qualities are calculated from the ratio of the dose equivalent to the absorbed close.

The calculated mean quality factor, averaged over all depths and angles, is $4.96 \pm 0.01$ when both neutrons and photons are considered, and $5.73 \pm 0.02$ when only neutron are included. The measured mean quality factor, averaged over all measured depths and angles, is $6.76 \pm 0.02$ for all events. The measured quality factors are determined using Equation 2.19 which is based upon event size to radiation quality relationships. This relationship is unchanged by the recent ICRP recommendations $\{59 \mid$. The LCS calculated quality factors are determined through the ratio dose equivalent and absorbed dose response functions, weighted by the neutron energy spectrum. Recent ICRP recommendations $[5,5,59 \mid$ suggest. doubling of this value. By comparing our measured mean radiation quality with that derived from the LCS Monte Carlo calculations, the factor by which the radiation quality factor for neutrons needs to be increased to agree with the event 


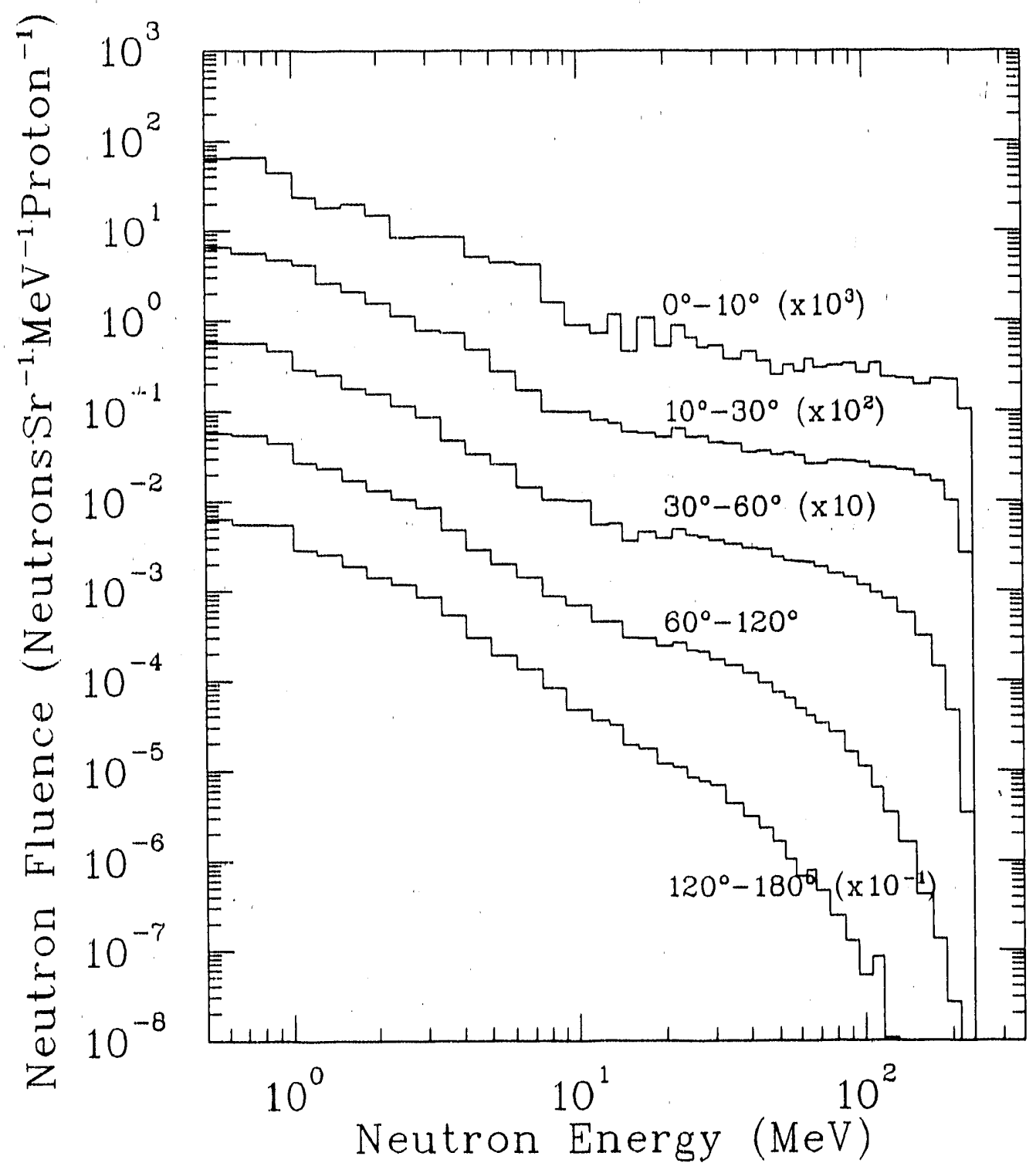

Figure 4.11: Computed neutron spectrum crossing from air into concrete for $230 \mathrm{MeV}$ protons incident upon an Fe target. 


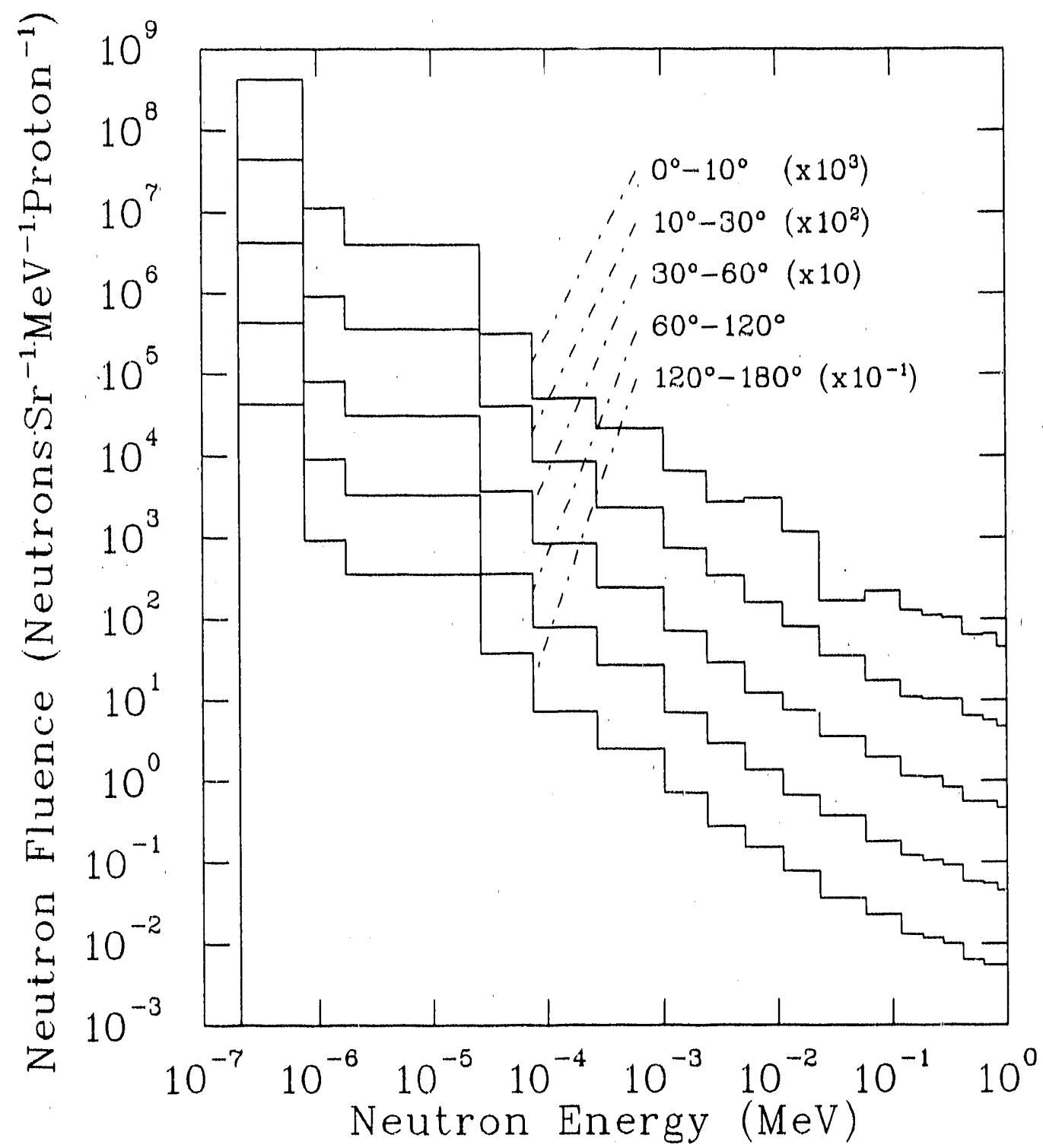

Figure 4.12: Computed low energy neutron spectrum crossing from air into concrete for $230 \mathrm{MeV}$ protons incident upon an Fe target. 


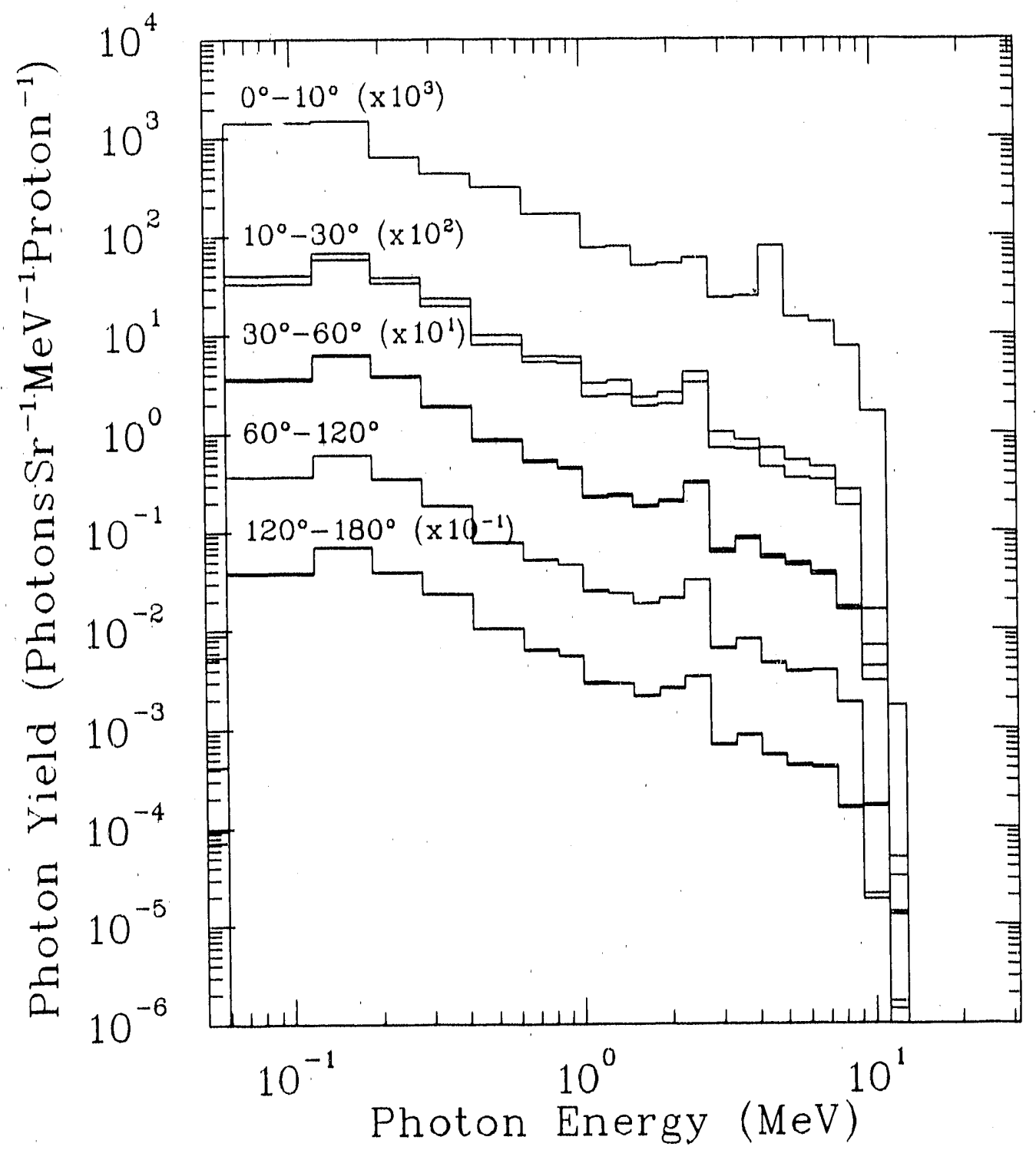

Figure 4.13: Photon spectra crossing into the concrete for $230 \mathrm{MeV}$ protons incident upon an Fe target calculated using LCS.S. 


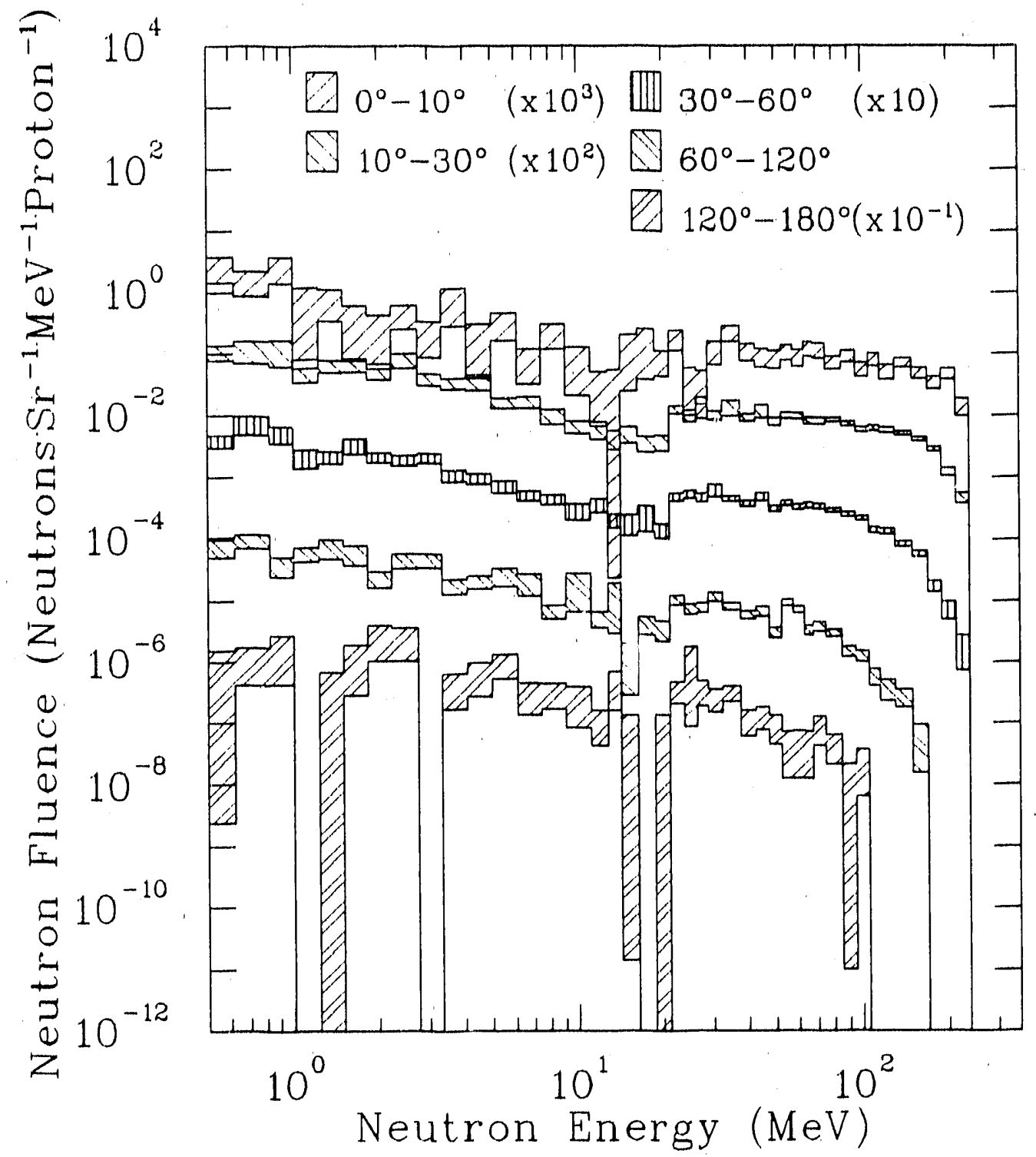

Figure 4.14: LCS calculated neutron spectra $105 \mathrm{~cm}$ in the concrete for $230 \mathrm{MeV}$ protons incident upon an Fe target. Filled areas represent values $\pm 1 \cdot \sigma$. 


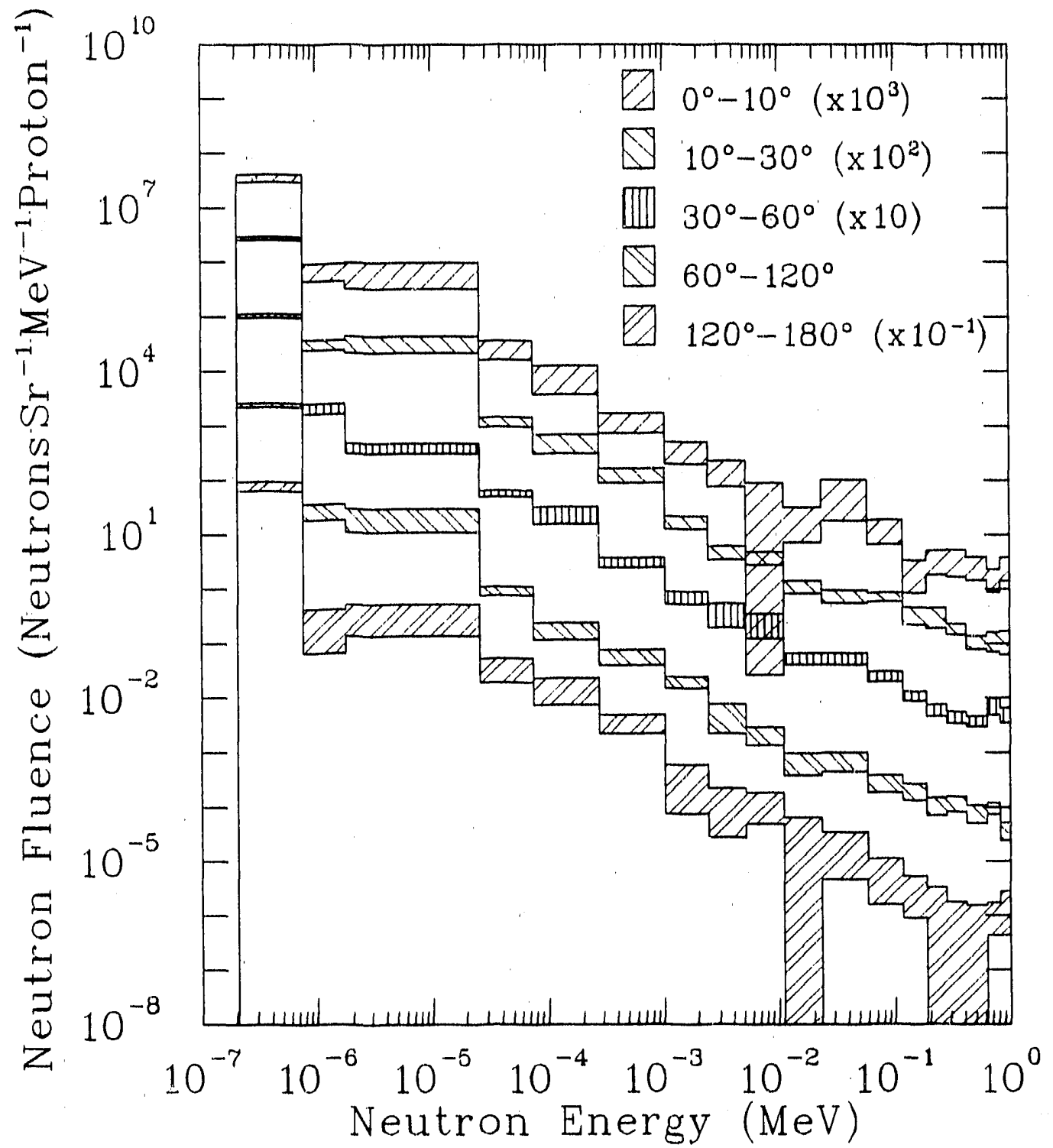

Figure 4.15: LCS calculated low energy neutron spectra $105 \mathrm{~cm}$ in the concrete for $230 \mathrm{MeV}$ protons incident upon an Fe target. Filled areas represent values $\pm 1 \cdot \sigma$. 


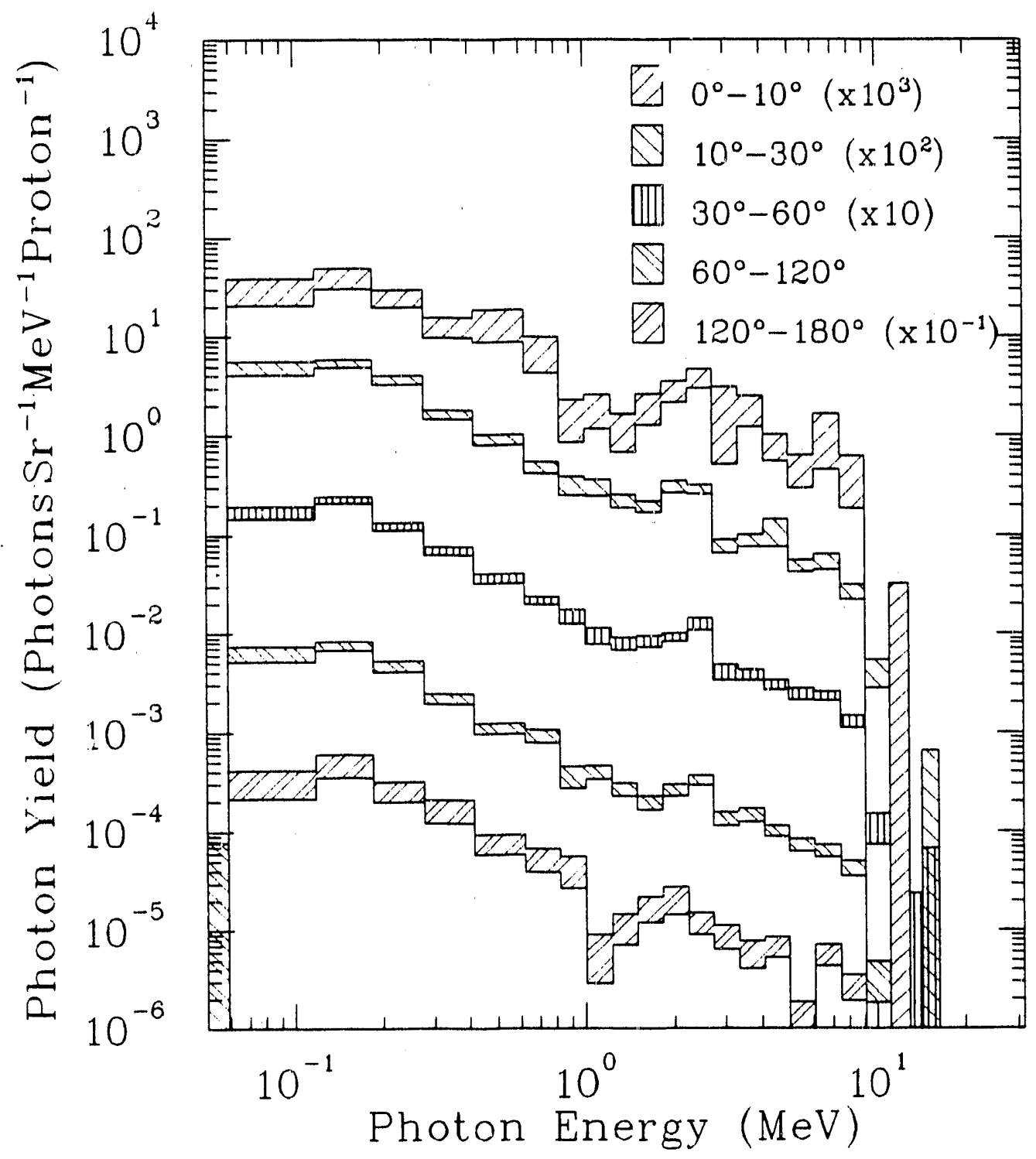

Figure 4.16: LCS calculated photon spectra $105 \mathrm{~cm}$ in the concrete for $230 \mathrm{MeV}$ protons incident upon an Fe target. Filled areas represent values $\pm 1 \cdot \sigma$. 
size quality factor relationships is determined. Based upon our measurements and calculations, the response function, averaged over this neutron spectrum, underestimates the radiation quality factor for neutrons by a factor of 1.36. Doubling of the radiation quality factor neutrons, per ICRP recommendations, results in overestimation of the radiation quality by a factor of 1.5 .

Comparisons between LCS computed and measured absorbed dose values are not presented here since they are similar to the dose equivalent comparisons. This is due to the relatively constant radiation qualities observed in both the calculated values and measured data (See Tables 3.2 and 4.15). Hence, the ratio of calculated to measured absorbed dose values differs from the ratio of the dose equivalent values by the ratio of the quality factors, 1.36 .

For the comparison of computed attenuation lengths with measured values, dose equivalent values are also calculated over angular intervals which closely bracketed measurement angles. Only shield penetrations between 60 and $210 \mathrm{~cm}$ of concrete are included to compare these calculations with the measured values over equal regions. Before $60 \mathrm{~cm}$ of concrete, low energy neutrons contribute significantly to dose values. Beyond $210 \mathrm{~cm}$, few measurements were made and the calculational statistics are poor. Figure 4.18 shows a plot of LCS computed values in angular intervals surrounding measurements angles, the results of a least squares fit to the LCS computed values in each angular region, and the measured dose equivalent values for the Fe target. The LCSS calculations are observed to overestimate the dose equivalent for all angles where measurements were performed. Since the calculated radiation quality is below the measured value, the 


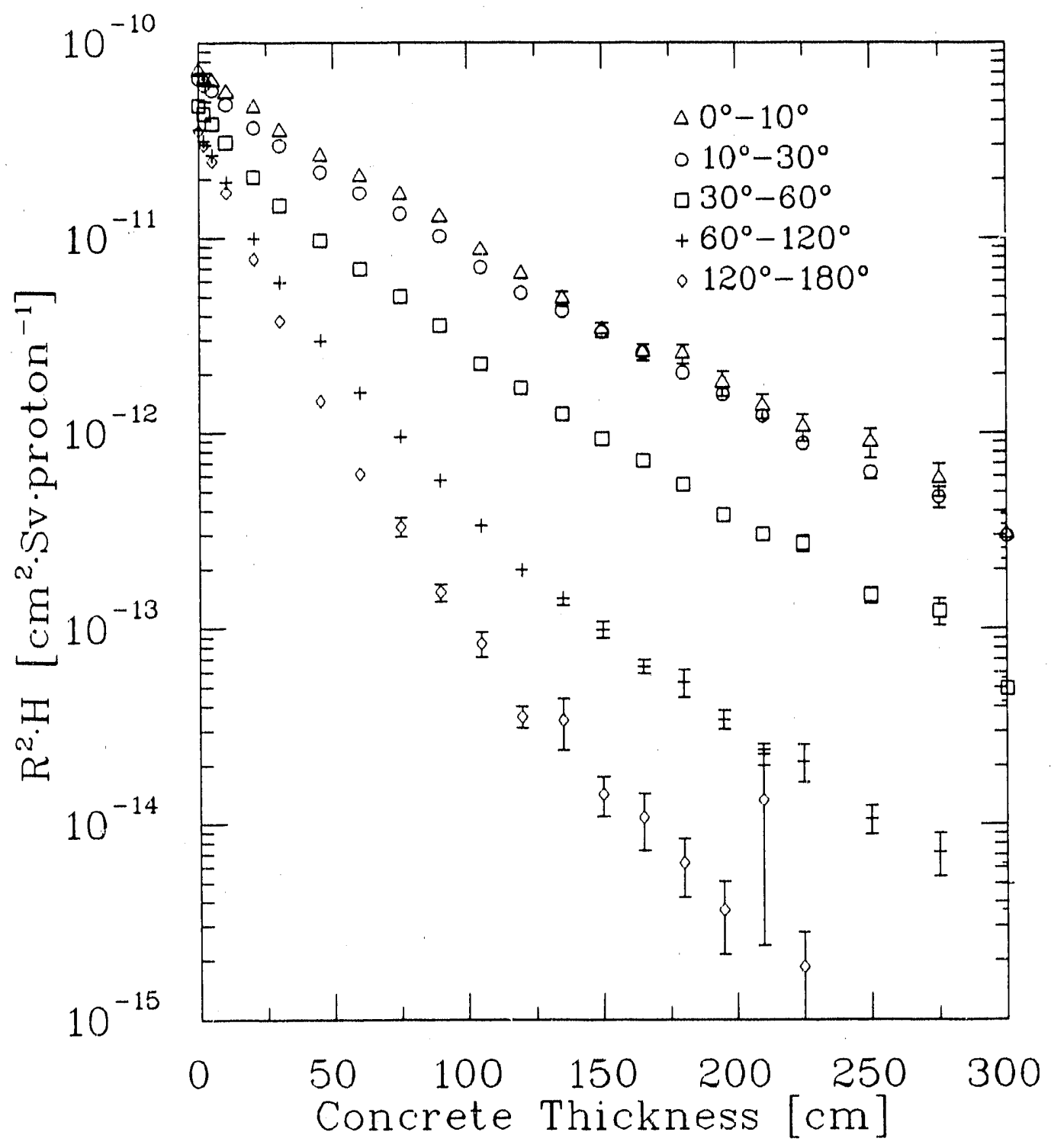

Figure 4.17: Dose equivalent per proton multiplied by the distance between the target and recording point squared versus the concrete thickness traversed. Values are computed using LCS for $230 \mathrm{MeV}$ protons incident upon an Fe target. in several angular regions. 


\begin{tabular}{||l|c|c|c|c|c||}
\hline \multicolumn{3}{|c|}{ Experiment } & \multicolumn{3}{c||}{ LCS } \\
\hline Angle & $\begin{array}{c}\mathrm{P} \\
\left(\mathrm{pSv} \cdot \mathrm{cm}^{2} \cdot \mathrm{p}^{-1}\right)\end{array}$ & $\begin{array}{c}\lambda \\
(\mathrm{cm})\end{array}$ & Angle & $\begin{array}{c}\mathrm{P} \\
\left(\mathrm{pSv} \cdot \mathrm{cm}^{2} \cdot \mathrm{p}^{-1}\right)\end{array}$ & $\begin{array}{c}\lambda \\
(\mathrm{cm})\end{array}$ \\
\hline $0^{\circ}$ & $64 \pm 7$ & $47.1 \pm 1.5$ & $0^{\circ}-10^{\circ}$ & $63 \pm 5$ & $54.0 \pm 1.6$ \\
$22^{\circ}$ & $34 \pm 3$ & $45.4 \pm 1.6$ & $10^{\circ}-30^{\circ}$ & $47.6 \pm 2.1$ & $56.7 \pm 1.0$ \\
$45^{\circ}$ & $13.4 \pm 1.2$ & $41.3 \pm 1.3$ & $40^{\circ}-50^{\circ}$ & $21.1 \pm 1.3$ & $49.4 \pm 1.1$ \\
$90^{\circ}$ & $4.08 \pm 1.0$ & $30.8 \pm 2.2$ & $85^{\circ}-95^{\circ}$ & $7.86 \pm 1.9$ & $31.4 \pm 1.8$ \\
\hline
\end{tabular}

Table 4.12: Comparison of measured attenuation parameters and those calculated using LCS for $230 \mathrm{MeV}$ protons incident upon an Fe target. The LCS results are from a fit to concrete depths ranging from $60 \mathrm{~cm}$ to $210 \mathrm{~cm}$ in each angular interval.

quality factor correction will increase the LCS overestimation. Table $4.12 \mathrm{com}-$ pares fitting parameters for measured and computed close equivalent values. LCS calculations predict the production term quite well at $0^{\circ}$ and $90^{\circ}$, however at $22^{\circ}$ and $45^{\circ}$ LCS over-predicts. Calculated dose equivalent attenuation lengths are larger than measured values at $0^{\circ}, 22^{\circ}$ and $45^{\circ}$, but are in agreement at $90^{\circ}$. Curiously enough, the LCS calculated attenuation length at $22^{\circ}$ is equal to the value at $0^{\circ}$. Ratios of LCS computed dose equivalent values to those measured are plotted in Figure 4.19.

Angular dependencies in production and attenuation lengths are shown in Tables 4.13-4.14, which list values determined for fitting over a number of depth and angular intervals. Other than the depression in attenuation length at $0^{\circ}$, attenuation is observed to fall off smoothly with angle. The depression at $0^{\circ}$ is most likely caused by low energy components persisting, to greater depths (Figure 4.17). Fits at greater penetrations show the (1) attenuation length ap)proaching, and exceeding the $22^{\circ}$ attenuation lengths as expected. The variations 


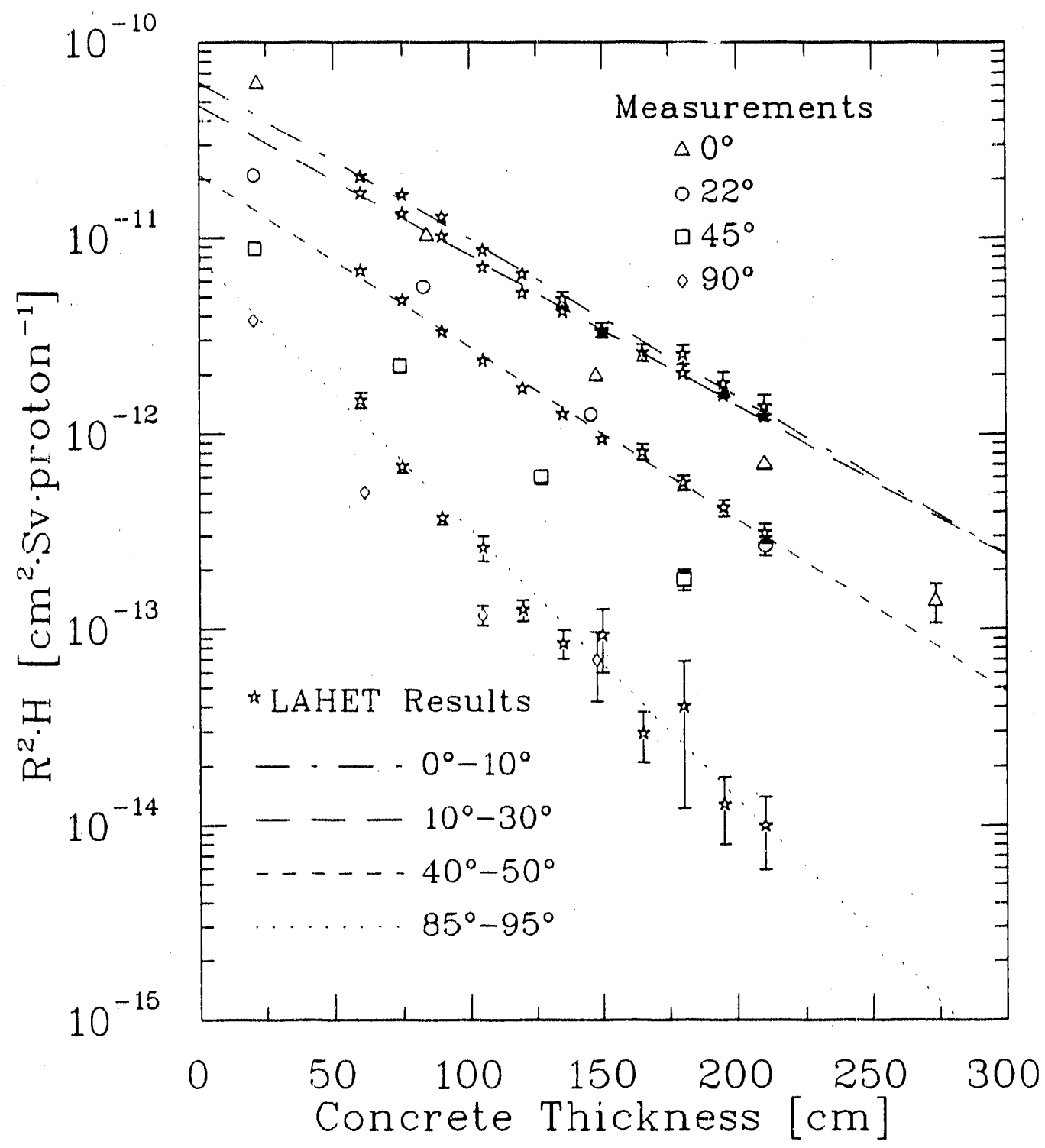

Figure 4.18: LCS computed dose equivalent per proton multiplied by the distance between target and recording point squared versus the concrete thickness in several angular regions. Also plotted are least squares fits to the results and measured data. 


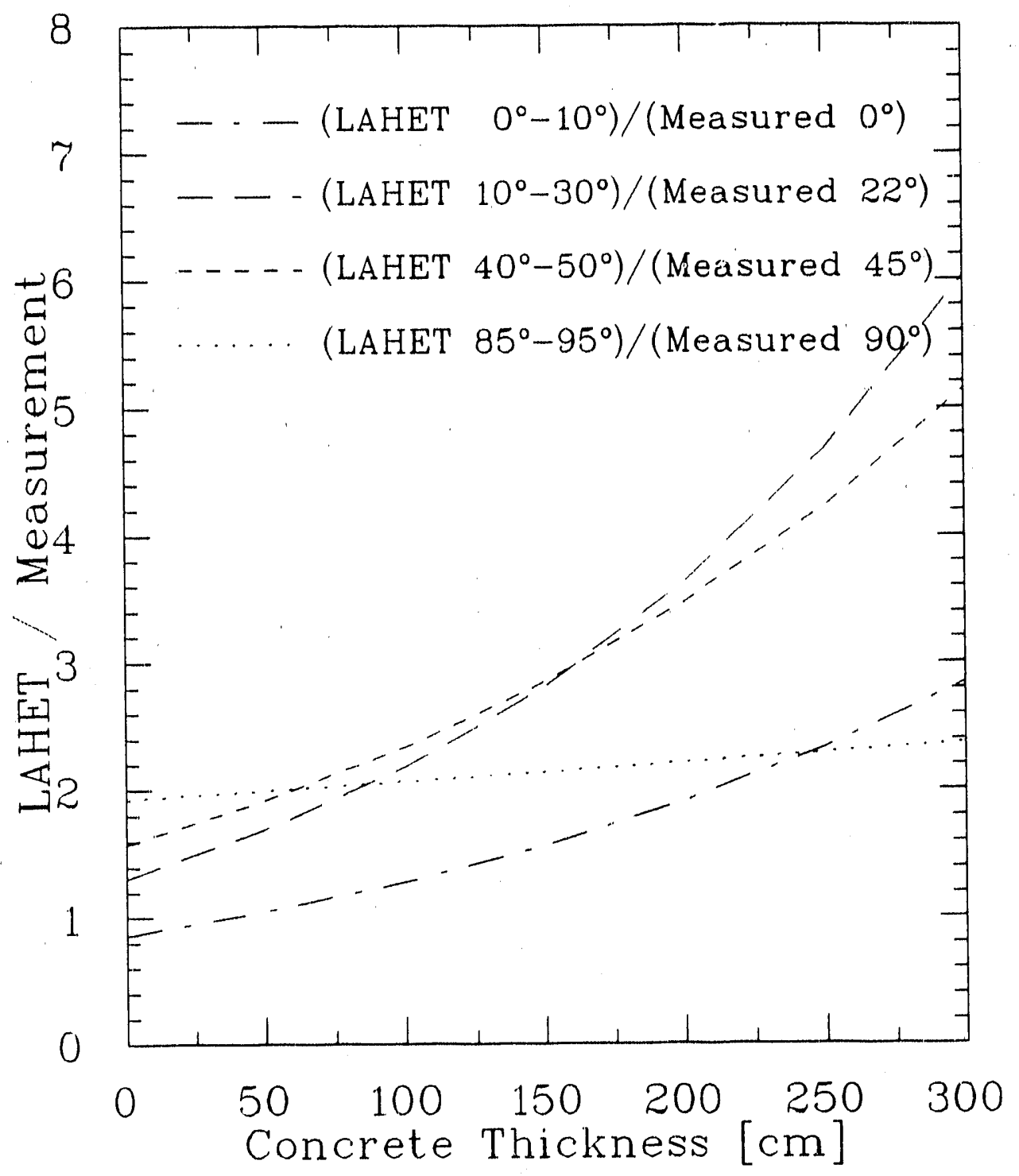

Figure 4.19: Ratio of calculated dose equivalent to measured dose equivalent values versus shield thickness. Ratio is taken from fitted parameters. 


\begin{tabular}{|c|c|c|}
\hline Angle & $\begin{array}{c}\text { Production } \\
\left(\mathrm{pSv} \cdot \mathrm{cm}^{2} \cdot \mathrm{p}^{-1}\right)\end{array}$ & $\begin{array}{l}\text { Attenuation } \\
\qquad(\mathrm{cm})\end{array}$ \\
\hline \multicolumn{3}{|c|}{ Fits on Depths $60-210 \mathrm{~cm}$} \\
\hline $0^{\circ}-30^{\circ}$ & $49.3 \pm 2.2$ & $56.4 \pm 1.1$ \\
\hline $30^{\circ}-60^{\circ}$ & $23.0 \pm 1.4$ & $47.5 \pm 1.0$ \\
\hline $60^{\circ}-120^{\circ}$ & $72.6 \pm 0.9$ & $35.6 \pm 1.2$ \\
\hline $0^{\circ}-10^{\circ}$ & $63 \pm 5$ & $54.0 \pm 1.6$ \\
\hline $10^{\circ}-30^{\circ}$ & $47.6 \pm 2.1$ & $56.7 \pm 1.0$ \\
\hline $30^{\circ}-40^{\circ}$ & $33.4 \pm 2.1$ & $48.9 \pm 1.1$ \\
\hline $40^{\circ} .50^{\circ}$ & $21.1 \pm 1.3$ & $49.4 \pm 1.1$ \\
\hline $50^{\circ}-60^{\circ}$ & $18.1 \pm 1.8$ & $42.7 \pm 1.4$ \\
\hline $60^{\circ}-75^{\circ}$ & $11.0 \pm 1.1$ & $40.3 \pm 1.2$ \\
\hline $70^{\circ}-85^{\circ}$ & $11.2 \pm 1.5$ & $31.4 \pm 1.0$ \\
\hline $85^{\circ}-95^{\circ}$ & $7.9 \pm 1.9$ & $31.4 \pm 1.8$ \\
\hline $95^{\circ}-105^{\circ}$ & $7.4 \pm 2.2$ & $27.7 \pm 1.7$ \\
\hline $105^{\circ}-120^{\circ}$ & $4.0 \pm 1.2$ & $31.1 \pm 2.1$ \\
\hline $120^{\circ}-180^{\circ}$ & $2.7 \pm 1.3$ & $32 \pm 4$ \\
\hline \multicolumn{3}{|c|}{ Fits on Depths $60-300 \mathrm{~cm}$} \\
\hline $0^{\circ}-10^{\circ}$ & $55 \pm 4$ & $58.0 \pm 1.5$ \\
\hline $10^{\circ}-30^{\circ}$ & $43.9 \pm 1.9$ & $59.0 \pm 0.9$ \\
\hline $30^{\circ}-60^{\circ}$ & $19.6 \pm 1.6$ & $51.1 \pm 1.3$ \\
\hline $60^{\circ}-120^{\circ}$ & $49.2 \pm 0.8$ & $40.6 \pm 1.5$ \\
\hline $120^{\circ}-180^{\circ}$ & $2.3 \pm 0.8$ & $32.9 \pm 2.2$ \\
\hline
\end{tabular}

Table 4.13: Attenuation and production coefficients determined from fits to depths greater than $60 \mathrm{~cm}$ concrete using LCS calculations.

in the large angle attenuation lengths for different fitting depths are due to the poor statistics at the large angles.

A comparison of the results for different angular intervals indicates that effective angles are weighted strongly toward the smaller of the angles in the interval. This is likely caused by the strong angular dependence of the neutron production as evidenced in Figure 4.20. Angle averaging techniques that are typically 


\begin{tabular}{|c|c|c|}
\hline Angle & $\begin{array}{l}\text { Production } \\
\left(\mathrm{pSv} \cdot \mathrm{cm}^{2} \cdot \mathrm{p}^{-1}\right)\end{array}$ & $\begin{array}{c}\text { Attenuation } \\
(\mathrm{cm})\end{array}$ \\
\hline \multicolumn{3}{|c|}{ Fits on Depths $105-210 \mathrm{~cm}$} \\
\hline $0^{\circ}-30^{\circ}$ & $40.0 \pm 1.3$ & $60.5 \pm 0.8$ \\
\hline $30^{\circ}-60^{\circ}$ & $17.0 \pm 0.7$ & $52.0 \pm 0.7$ \\
\hline $60^{\circ}-120^{\circ}$ & $40.8 \pm 0.5$ & $40.6 \pm 1.2$ \\
\hline $0^{\circ}-10^{\circ}$ & $50 \pm 7$ & $58 \pm 3$ \\
\hline $10^{\circ}-30^{\circ}$ & $.38 .8 \pm 1.1$ & $60.9 \pm 0.6$ \\
\hline $30^{\circ}-40^{\circ}$ & $25.6 \pm 1.9$ & $53.0 \pm 1.3$ \\
\hline $40^{\circ}-50^{\circ}$ & $16.6 \pm 1.3$ & $5.3 .1 \pm 1.4$ \\
\hline $50^{\circ}-60^{\circ}$ & $11.3 \pm 1.0$ & $48.5 \pm 1.4$ \\
\hline $60^{\circ}-75^{\circ}$ & $6.9 \pm 0.8$ & $45.4 \pm 1.4$ \\
\hline $70^{\circ}-85^{\circ}$ & $6.5 \pm 1.3$ & $35.0 \pm 1.6$ \\
\hline $85^{\circ}-95^{\circ}$ & $5.4 \pm 2.8$ & $34 \pm 4$ \\
\hline $95^{\circ}-105^{\circ}$ & $3.1 \pm 2.0$ & $32 \pm 4$ \\
\hline $105^{\circ}-120^{\circ}$ & $1.5 \pm 0.8$ & $38 \pm 5$ \\
\hline $120^{\circ}-180^{\circ}$ & $0.55 \pm 0.52$ & $45 \pm 12$ \\
\hline \multicolumn{3}{|c|}{ Fits on Depths $105-300 \mathrm{~cm}$} \\
\hline $0^{\circ}-10^{\circ}$ & $43 \pm 4$ & $61.9 \pm 2.0$ \\
\hline $10^{\circ}-30^{\circ}$ & $37.2 \pm 1.5$ & $61.8 \pm 0.8$ \\
\hline $30^{\circ}-60^{\circ}$ & $15.0 \pm 1.6$ & $54.5 \pm 1.6$ \\
\hline $60^{\circ}-120^{\circ}$ & $27.0 \pm 2.4$ & $45.9 \pm 1.4$ \\
\hline $120^{\circ}-180^{\circ}$ & $1.0 \pm 0.6$ & $38 \pm 4$ \\
\hline
\end{tabular}

Table 4.14: Attenuation and production coefficients determined from fits to depths greater than 105 cm concrete using LCS calculations. 
used to find effective angles for angular bins, such as Equation 4.2, result in an overestimation of contributions to dose equivalent at large angles. 


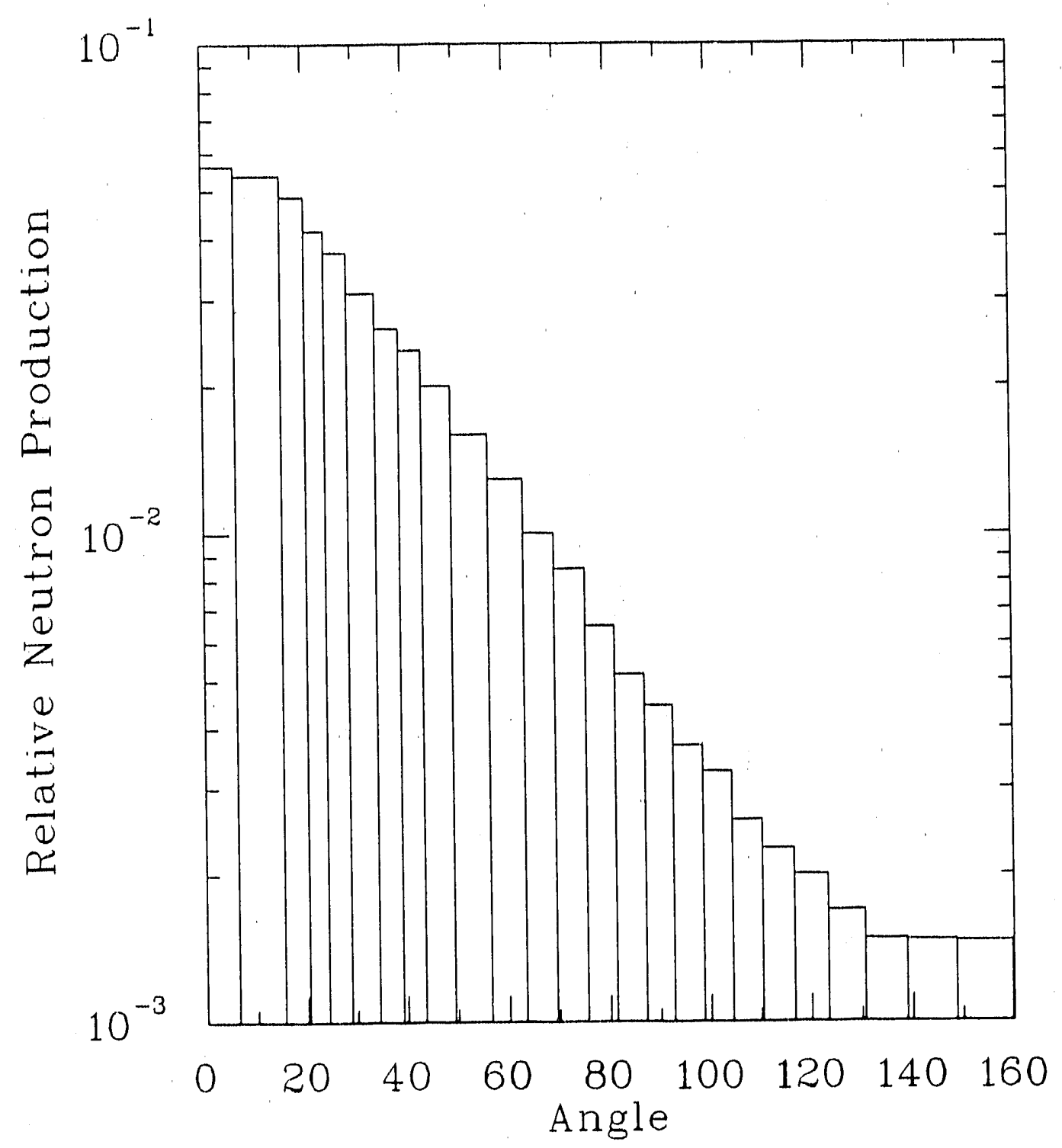

Figure 4.20: Neutron production in each angular interval as calculated by LCS. 


\begin{tabular}{|c|c|c|c|}
\hline $\begin{array}{l}\mathrm{D} \\
(\mathrm{cm})\end{array}$ & $\begin{array}{c}\mathrm{H} \\
\left(\mathrm{pSv} \cdot \mathrm{cm}^{2} \cdot \text { Proton }^{-1}\right)\end{array}$ & $\begin{array}{c}\text { D } \\
\left(\mathrm{pGy} \cdot \mathrm{cm}^{2} \cdot \mathrm{Proton}^{-1}\right)\end{array}$ & $Q$ \\
\hline 0 & $71.2 \pm 2.2$ & $14.5 \pm 0.3$ & $4.91 \pm 0.19$ \\
\hline 2 & $65.3 \pm 2.0$ & $12.8 \pm 0.3$ & $5.08 \pm 0.19$ \\
\hline 5 & $63.3 \pm 2.1$ & $12.6 \pm 0.3$ & $5.01 \pm 0.20$ \\
\hline 10 & $54.9 \pm 1.7$ & $11.5 \pm 0.3$ & $4.78 \pm 0.19$ \\
\hline 20 & $46.3 \pm 1.8$ & $9.72 \pm 0.28$ & $4.76 \pm 0.22$ \\
\hline 30 & $35.1 \pm 1.2$ & $7.59 \pm 0.22$ & $4.62 \pm 0.20$ \\
\hline 45 & $26.2 \pm 0.9$ & $5.68 \pm 0.17$ & $4.60 \pm 0.21$ \\
\hline 60 & $20.6 \pm 0.9$ & $4.40 \pm 0.16$ & $4.68 \pm 0.26$ \\
\hline 75 & $16.6 \pm 0.8$ & $3.52 \pm 0.14$ & $4.72 \pm 0.29$ \\
\hline 90 & $12.8 \pm 0.8$ & $2.66 \pm 0.14$ & $4.8 \pm 0.4$ \\
\hline 105 & $8.7 \pm 0.6$ & $1.90 \pm 0.11$ & $4.6 \pm 0.4$ \\
\hline 120 & $6.5 \pm 0.5$ & $1 . .42 \pm 0.09$ & $4.6 \pm 0.4$ \\
\hline 135 & $4.9 \pm 0.4$ & $1.06 \pm 0.08$ & $4.6 \pm 0.5$ \\
\hline 150 & $3.4 \pm 0.3$ & $0.76 \pm 0.06$ & $4.5 \pm 0.5$ \\
\hline 165 & $2.60 \pm 0.26$ & $0.59 \pm 0.05$ & $4.4 \pm 0.6$ \\
\hline 180 & $2.55 \pm 0.29$ & $0.55 \pm 0.06$ & $4.6 \pm 0.7$ \\
\hline 195 & $1.81 \pm 0.26$ & $0.41 \pm 0.06$ & $4.4 \pm 0.9$ \\
\hline 210 & $1.38 \pm 0.20$ & $0.31 \pm 0.04$ & $4.5 \pm 0.9$ \\
\hline 225 & $1.07 \pm 0.17$ & $0.24 \pm 0.04$ & $4.5 \pm 1.0$ \\
\hline 250 & $0.90 \pm 0.15$ & $0.19 \pm 0.03$ & $4.6 \pm 1.1$ \\
\hline 275 & $0.58 \pm 0.11$ & $0.127 \pm 0.024$ & $4.6 \pm 1.2$ \\
\hline 300 & $0.31 \pm 0.08$ & $0.071 \pm 0.018$ & $4.3 \pm 1.5$ \\
\hline 325 & $0.32 \pm 0.16$ & $0.08 \pm 0.04$ & $4.2 \pm 2.9$ \\
\hline 350 & $0.13 \pm 0.05$ & $0.030 \pm 0.011$ & $4.5 \pm 2.4$ \\
\hline 400 & $0.015 \pm 0.015$ & $0.004 \pm 0.004$ & $3.9 \pm 5$ \\
\hline
\end{tabular}

Table 4.15: Dose equivalent, quality factors, and dose calculated in $0^{\circ}-10^{\circ}$ interval using LCS. 


\begin{tabular}{||l|c|c|c||}
\hline $\begin{array}{l}\mathrm{D} \\
(\mathrm{cm})\end{array}$ & $\begin{array}{c}\mathrm{H} \\
\left(\mathrm{pSv} \cdot \mathrm{cm}^{2} \cdot \text { Proton }^{-1}\right)\end{array}$ & $\begin{array}{c}\mathrm{D} \\
\left(\mathrm{pGy} \cdot \mathrm{cm}^{2} \cdot \text { Proton }^{-1}\right)\end{array}$ & $\mathrm{Q}$ \\
\hline 0 & $65.0 \pm 1.0$ & $13.4 \pm 0.14$ & $4.84 \pm 0.08$ \\
2 & $60.5 \pm 0.9$ & $11.7 \pm 0.12$ & $5.18 \pm 0.09$ \\
5 & $56.7 \pm 0.8$ & $11.2 \pm 0.12$ & $5.07 \pm 0.09$ \\
10 & $48.1 \pm 0.7$ & $9.83 \pm 0.11$ & $4.88 \pm 0.08$ \\
20 & $36.5 \pm 0.5$ & $7.67 \pm 0.09$ & $4.76 \pm 0.08$ \\
30 & $29.7 \pm 0.5$ & $6.24 \pm 0.08$ & $4.74 \pm 0.09$ \\
45 & $21.7 \pm 0.4$ & $4.61 \pm 0.06$ & $4.70 \pm 0.09$ \\
60 & $16.9 \pm 0.3$ & $3.59 \pm 0.06$ & $4.71 \pm 0.11$ \\
75 & $13.3 \pm 0.3$ & $2.80 \pm 0.06$ & $4.75 \pm 0.12$ \\
90 & $10.2 \pm 0.3$ & $2.13 \pm 0.04$ & $4.79 \pm 0.14$ \\
105 & $7.07 \pm 0.20$ & $1.53 \pm 0.04$ & $4.63 \pm 0.17$ \\
120 & $5.24 \pm 0.15$ & $1.13 \pm 0.03$ & $4.64 \pm 0.17$ \\
135 & $4.21 \pm 0.15$ & $0.905 \pm 0.028$ & $4.64 \pm 0.21$ \\
150 & $3.31 \pm 0.14$ & $0.704 \pm 0.026$ & $4.69 \pm 0.25$ \\
165 & $2.59 \pm 0.12$ & $0.552 \pm 0.023$ & $4.69 \pm 0.28$ \\
180 & $2.03 \pm 0.09$ & $0.426 \pm 0.018$ & $4.77 \pm 0.29$ \\
195 & $1.58 \pm 0.09$ & $0.329 \pm 0.017$ & $4.8 \pm 0.4$ \\
210 & $1.23 \pm 0.08$ & $0.263 \pm 0.016$ & $4.7 \pm 0.4$ \\
225 & $0.88 \pm 0.06$ & $0.190 \pm 0.011$ & $4.6 \pm 0.4$ \\
250 & $0.63 \pm 0.05$ & $0.135 \pm 0.010$ & $4.7 \pm 0.5$ \\
275 & $0.47 \pm 0.06$ & $0.097 \pm 0.010$ & $4.9 \pm 0.8$ \\
300 & $0.30 \pm 0.04$ & $0.063 \pm 0.008$ & $4.7 \pm 0.9$ \\
325 & $0.296 \pm 0.011$ & $0.059 \pm 0.020$ & $5.0 \pm 2.4$ \\
350 & $0.103 \pm 0.018$ & $0.024 \pm 0.004$ & $4.4 \pm 1.1$ \\
400 & $0.054 \pm 0.012$ & $0.014 \pm 0.003$ & $3.8 \pm 1.2$ \\
\hline
\end{tabular}

Table 4.16: Dose equivalent, quality factors, and dose calculated in $10^{\circ}-30^{\circ} \mathrm{in}-$ terval using LCS. 


\begin{tabular}{|c|c|c|c|}
\hline $\begin{array}{l}\mathrm{D} \\
(\mathrm{cm}) \\
\end{array}$ & $\begin{array}{c}\mathrm{H} \\
\left(\mathrm{pSv} \cdot \mathrm{cm}^{2} \cdot \text { Proton }^{-1}\right)\end{array}$ & $\begin{array}{c}\mathrm{D} \\
\left(\mathrm{pGy} \cdot \mathrm{cm}^{2} \cdot \operatorname{Proton}^{-1}\right)\end{array}$ & $Q$ \\
\hline 0 & $47.4 \pm 0.5$ & $9.09 \pm 0.08$ & $5.21 \pm 0.07$ \\
\hline 2 & $42.9 \pm 0.5$ & $7.87 \pm 0.07$ & $5.45 \pm 0.07$ \\
\hline 5 & $38.3 \pm 0.4$ & $7.23 \pm 0.06$ & $5.30 \pm 0.07$ \\
\hline 10 & $30.8 \pm 0.4$ & $6.08 \pm 0.06$ & $5.06 \pm 0.07$ \\
\hline 20 & $20.4 \pm 0.3$ & $4.21 \pm 0.04$ & $4.85 \pm 0.07$ \\
\hline 30 & $14.7 \pm 0.2$ & $3.05 \pm 0.03$ & $4.83 \pm 0.08$ \\
\hline 45 & $9.69 \pm 0.15$ & $2.00 \pm 0.03$ & $4.85 \pm 0.09$ \\
\hline 60 & $6.93 \pm 0.12$ & $1.41 \pm 0.02$ & $4.91 \pm 0.10$ \\
\hline 75 & $5.02 \pm 0.11$ & $1.02 \pm 0.02$ & $4.92 \pm 0.13$ \\
\hline 90 & $3.55 \pm 0.08$ & 0.723 土 0.016 & $4.91 \pm 0.15$ \\
\hline 105 & $2.26 \pm 0.06$ & $0.466 \pm 0.011$ & $4.85 \pm 0.16$ \\
\hline 120 & $1.70 \pm 0.05$ & $0.347 \pm 0.010$ & $4.89 \pm 0.20$ \\
\hline 1.35 & $1.25 \pm 0.05$ & $0.256 \pm 0.009$ & $4.87 \pm 0.25$ \\
\hline 150 & $0.94 \pm 0.04$ & $0.191 \pm 0.007$ & $4.90 \pm 0.26$ \\
\hline 165 & $0.72 \pm 0.04$ & $0.147 \pm 0.006$ & $4.9 \pm 0.3$ \\
\hline 180 & $0.544 \pm 0.028$ & $0.111 \pm 0.005$ & $4.9 \pm 0.3$ \\
\hline 195 & $0.380 \pm 0.021$ & $0.079 \pm 0.004$ & $4.8 \pm 0.4$ \\
\hline 210 & $0.305 \pm 0.019$ & $0.062 \pm 0.004$ & $4.9 \pm 0.4$ \\
\hline 225 & $0.272 \pm 0.026$ & $0.054 \pm 0.005$ & $5.1 \pm 0.7$ \\
\hline 250 & $0.148 \pm 0.013$ & $0.0 .307 \pm 0.0025$ & $4.8 \pm 0.6$ \\
\hline 275 & $0.123 \pm 0.019$ & $0.0228 \pm 0.0029$ & $5.4 \pm 1.1$ \\
\hline 300 & $0.049 \pm 0.007$ & $0.0104 \pm 0.0014$ & $4.8 \pm 0.9$ \\
\hline 325 & $0.033 \pm 0.006$ & $0.0071 \pm 0.0011$ & $4.7 \pm 1.1$ \\
\hline 350 & $0.019 \pm 0.004$ & $0.0038 \pm 0.0008$ & $4.9 \pm 1.6$ \\
\hline 400 & $0.0046 \pm 0.0020$ & $0.001 .2 \pm 0.0005$ & $3.8 \pm 2.4$ \\
\hline
\end{tabular}

Table 4.17: Dose equivalent, quality factors, and dose calculated in $30^{\circ}-60^{\circ}$ interval using LCS. 


\begin{tabular}{||l|c|c|c||}
\hline $\begin{array}{l}\mathrm{D} \\
(\mathrm{cm})\end{array}$ & $\begin{array}{c}\mathrm{H} \\
\left(\mathrm{pSv} \cdot \mathrm{cm}^{2} \cdot \text { Proton }^{-1}\right)\end{array}$ & $\begin{array}{c}\mathrm{D} \\
\left(\mathrm{pGy} \cdot \mathrm{cm}^{2} \cdot \text { Proton }^{-1}\right)\end{array}$ & $\mathrm{Q}$ \\
\hline 0 & $35.9 \pm 0.4$ & $6.96 \pm 0.05$ & $5.15 \pm 0.06$ \\
2 & $31.3 \pm 0.3$ & $5.62 \pm 0.04$ & $5.56 \pm 0.06$ \\
5 & $26.5 \pm 0.3$ & $4.96 \pm 0.04$ & $5.34 \pm 0.06$ \\
10 & $19.2 \pm 0.2$ & $3.85 \pm 0.03$ & $4.99 \pm 0.06$ \\
20 & $9.96 \pm 0.13$ & $2.19 \pm 0.02$ & $4.55 \pm 0.07$ \\
30 & $5.95 \pm 0.09$ & $1.31 \pm 0.02$ & $4.53 \pm 0.08$ \\
45 & $2.97 \pm 0.07$ & $0.648 \pm 0.010$ & $4.58 \pm 0.12$ \\
60 & $1.61 \pm 0.05$ & $0.349 \pm 0.007$ & $4.60 \pm 0.16$ \\
75 & $0.953 \pm 0.028$ & $0.201 \pm 0.004$ & $4.73 \pm 0.17$ \\
90 & $0.571 \pm 0.019$ & $0.119 \pm 0.003$ & $4.80 \pm 0.20$ \\
105 & $0.336 \pm 0.016$ & $0.071 \pm 0.003$ & $4.8 \pm 0.3$ \\
120 & $0.199 \pm 0.010$ & $0.0413 \pm 0.0018$ & $4.8 \pm 0.3$ \\
135 & $0.142 \pm 0.011$ & $0.0293 \pm 0.0019$ & $4.9 \pm 0.5$ \\
150 & $0.099 \pm 0.010$ & $0.0196 \pm 0.0016$ & $5.1 \pm 0.6$ \\
165 & $0.064 \pm 0.005$ & $0.013 \pm 0.009$ & $5.0 \pm 0.6$ \\
180 & $0.053 \pm 0.009$ & $0.0105 \pm 0.0015$ & $5.1 \pm 1.1$ \\
195 & $0.034 \pm 0.004$ & $0.0069 \pm 0.0007$ & $5.0 \pm 0.7$ \\
210 & $0.023 \pm 0.003$ & $0.0045 \pm 0.0005$ & $5.1 \pm 0.9$ \\
225 & $0.021 \pm 0.005$ & $0.0039 \pm 0.0007$ & $5.4 \pm 1.5$ \\
250 & $0.0106 \pm 0.0018$ & $0.0021 \pm 0.0003$ & $5.0 \pm 1.2$ \\
275 & $0.0072 \pm 0.0018$ & $0.0014 \pm 0.0003$ & $5.2 \pm 1.7$ \\
300 & $0.0050 \pm 0.0013$ & $0.0010 \pm 0.0002$ & $5.0 \pm 1.8$ \\
325 & $0.009 \pm 0.006$ & $0.0015 \pm 0.0010$ & $5.6 \pm 5.4$ \\
350 & $0.0027 \pm 0.0009$ & $0.00055 \pm 0.00018$ & $4.9 \pm 2.3$ \\
400 & $0.0005 \pm 0.0004$ & $0.00013 \pm 0.00009$ & $4.2 \pm 4.2$ \\
\hline
\end{tabular}

Table 4.18: Dose equivalent, quality factors, and dose calculated in $60^{\circ}-120^{\circ}$ interval using LCS. 


\begin{tabular}{|c|c|c|c|}
\hline $\begin{array}{l}\mathrm{D} \\
(\mathrm{cm})\end{array}$ & $\begin{array}{c}\mathrm{H} \\
\left(\mathrm{pSv} \cdot \mathrm{cm}^{2} \cdot \text { Proton }^{-1}\right)\end{array}$ & $\begin{array}{c}D \\
\left(\mathrm{pGy} \cdot \mathrm{cm}^{2} \cdot \text { Proton }^{-1}\right)\end{array}$ & $Q$ \\
\hline 0 & $35.1 \pm 0.5$ & $6.91 \pm 0.06$ & $5.07 \pm 0.08$ \\
\hline 2 & $29.8 \pm 0.4$ & $5.37 \pm 0.05$ & $5.54 \pm 0.09$ \\
\hline 5 & $24.7 \pm 0.3$ & $4.71 \pm 0.05$ & $5.25 \pm 0.09$ \\
\hline 10 & $17.0 \pm 0.3$ & $3.53 \pm 0.04$ & $4.83 \pm 0.10$ \\
\hline 20 & $7.77 \pm 0.18$ & $1.82 \pm 0.03$ & $4.27 \pm 0.11$ \\
\hline 30 & $3.76 \pm 0.12$ & $0.957 \pm 0.018$ & $3.92 \pm 0.14$ \\
\hline 45 & $1.46 \pm 0.06$ & $0.378 \pm 0.010$ & $3.85 \pm 0.19$ \\
\hline 60 & $0.61 \pm 0.03$ & $0.160 \pm 0.005$ & $3.85 \pm 0.24$ \\
\hline 75 & $0.33 \pm 0.04$ & $0.078 \pm 0.005$ & $4.2 \pm 0.6$ \\
\hline 90 & $0.153 \pm 0.015$ & $0.0382 \pm 0.0026$ & $4.0 \pm 0.5$ \\
\hline 105 & $0.084 \pm 0.013$ & $0.0211 \pm 0.0023$ & $4.0 \pm 0.7$ \\
\hline 120 & $0.035 \pm 0.004$ & $0.0091 \pm 0.0009$ & $3.9 \pm 0.6$ \\
\hline 135 & $0.034 \pm 0.010$ & $0.0077 \pm 0.0016$ & $4.4 \pm 1.6$ \\
\hline 150 & $0.014 \pm 0.003$ & $0.0032 \pm 0.0006$ & $4.4 \pm 1.3$ \\
\hline 165 & $0.011 \pm 0.003$ & $0.0023 \pm 0.0006$ & $4.7 \pm 1.9$ \\
\hline 180 & $0.0063 \pm 0.0021$ & $0.001 .3 \pm 0.0004$ & $4.8 \pm 2.2$ \\
\hline 195 & $0.0036 \pm 0.0015$ & $0.0007 \pm 0.0003$ & $5.3 \pm .3$ \\
\hline 210 & $0.013 \pm 0.011$ & $0.003 \pm 0.002$ & $5.2 \pm 6$ \\
\hline 225 & $0.0019 \pm 0.0009$ & $0.00035 \pm 0.00017$ & $5.3 \pm 4$ \\
\hline 250 & $0.0007 \pm 0.0006$ & $0.00019 \pm 0.00012$ & $3.7 \pm 4$ \\
\hline 275 & $0.0006 \pm 0.0006$ & $0.00011 \pm 0.00011$ & $5.2 \pm 7$ \\
\hline 300 & $0.0006 \pm 0.0006$ & $0.00011 \pm 0.00010$ & $5.2 \pm 7$ \\
\hline 325 & $0.0006 \pm 0.0006$ & $0.00011 \pm 0.00010$ & $5.2 \pm 7$ \\
\hline 350 & $0.0006 \pm 0.0005$ & $0.00014 \pm 0.00010$ & $4.1 \pm 5$ \\
\hline
\end{tabular}

Table 4.19: Dose equivalent, quality factors, and dose calculated in $120^{\circ}-180^{\circ}$ interval using LCS. 


\section{Chapter 5}

\section{Conclusions}

Absorbed dose and dose equivalent values were measured and calculated as a function of depth in a thick concrete shield at neutron emission angles of $0^{\circ}, 22^{\circ}$, $45^{\circ}$, and $90^{\circ}$ for $230 \mathrm{MeV}$ protons incident upon a stopping length Fe target. To investigate material effects, $\mathrm{Al}$ and $\mathrm{Pb}$ target measurements were also made. Neutron attenuation properties were determined and compared with Monte Carlo neutron transport calculations. These data are useful for determining the shielding requirements for proton radiotherapy facilities and as a benchmark for future Monte Carlo calculations.

\subsection{Measurements}

Energy deposition. spectra produced by neutron induced interactions were measured with low pressure tissue-equivalent proportional counters. Event size spectra measured at depth in the shield were found to be independent of target material. As the observed radiation environment at depth in the shield is rominated by high energy neutrons, and is similar for each target material, measurements suggest that for $230 \mathrm{MeV}$ proton bombardment, high energy neutron production 
is material independent. High energy neutron emission was found to be forward peaked, while low energy neutron production was observed to be fairly isotropic and to increase significantly with target atomic number.

Absorbed dose and radiation quality were determined at numerous depths and angles in the concrete shielding. Values are listed in Tables 3.2-3:4. The average radiation quality factor determined was $6.76 \pm 0.02$.

Dose equivalent values measured at depth were used to determine the attenuation of the neutron spectrum. The similarity of the penetrating neutrons for different target materials allowed the combination of data from different target materials for precise attenuation length determination. Attenuation parameters as a function of angle for $230 \mathrm{MeV}$ proton bombardment of $\mathrm{Al}, \mathrm{Fe}$, and $\mathrm{Pb}$ targets are listed in Table 5.1.

\subsection{Monte Carlo Calculations}

Neutron dose and attenuation length values were compared with calculations based upon the High Energy Transport Code (HETC and LAHET) to determine their ability to predict experimental results. The Bertini intranuclear cascade model, as implemented in HETC was found to overestimate high energy neutrou production in the forward direction. This results in over-prediction of both the dose deposited anc' attenuation lengths. Other investigators have also reporter that HETC exaggerates absolute neutron yields.

At $0^{\circ}$, HETC-DO overestimates measurements by a factor of five at the shield entrance. HETC-DO calculations over-precicted dose equivalent values by a 


\begin{tabular}{|c|c|c|c|c|c|}
\hline & \multicolumn{5}{|c|}{$\begin{array}{l}\text { Production Term } \\
\left(\mathrm{pSv} \cdot \mathrm{cm}^{2} \cdot \mathrm{p}^{-1}\right)\end{array}$} \\
\hline Angle & Measured & LCS & HETC-DO & IAEA & Tesch \\
\hline $0^{\circ}$ & $63 \pm 7$ & $63 \pm 5$ & 372 & 87 & - \\
\hline $22^{\circ}$ & $33 \pm 4$ & $48 \pm 2$ & 129 & - & - \\
\hline $45^{\circ}$ & $13.4 \pm 1.2$ & $21.1 \pm 1.3$ & 43 & - & - \\
\hline $90^{\circ}$ & $4.1 \pm 1.0$ & $7.9 \pm 1.9$ & 4.9 & 16 & 4.5 \\
\hline & \multicolumn{5}{|c|}{$\begin{array}{l}\text { Attenuation Length } \\
\qquad(\mathrm{cm})\end{array}$} \\
\hline Angle & Measured & LCS & HETC-DO & IAEA & Tesch \\
\hline $0^{\circ}$ & $47.1 \pm 1.5$ & $54.0 \pm 1.6$ & 61.1 & 49 & - \\
\hline $22^{\circ}$ & $45.4 \pm 1.6$ & $56.7 \pm 1.0$ & 54.3 & - & - \\
\hline $45^{\circ}$ & $41.3 \pm 1.3$ & $49.4 \pm 1.1$ & 49.0 & - & - \\
\hline $90^{\circ}$ & $30.8 \pm 2.2$ & $31.4 \pm 1.8$ & 42.0 & 39 & 41 \\
\hline
\end{tabular}

Table 5.1: Summary of attenuation parameters measured, determined using the LCS and HETC-DO calculations, and using the estimation methods of the IAEA and Tesch. Attenuation lengths are given in $\mathrm{cm}$ of $\rho=1.88 \mathrm{~g} \cdot \mathrm{cm}^{-3}$ concrete. 
factor of twenty-five at $300 \mathrm{~cm}$ depth in concrete.

LAHET predicts the entrance dose equivalent quite well at $0^{\circ}$, however, by $300 \mathrm{~cm}$, it overestimates measurements by nearly a factor of three. At $90^{\circ}$, HETC-DO over-predicts the attenuation length, while LAHET predicts the proper attenuation length. Inclusion of the preequilibrium model in LAHET can be given the credit for this improvement. In all cases, LAHET calculations predict measurements better than density corrected HETC-DO calculations by nearly a factor of two.

Comparing the radiation quality estimated from the Monte Carlo calculations with our measured values indicates that the mean quality factor for neutrons should be increased. The ICRP has recently recommended doubling the quality factor [58], however our measurements suggest a factor of 1.36 is reasonable.

\subsection{Summary}

For proton radiation therapy facilities, the radiation shielding required to protect personnel from the penetrating high energy neutrons produced as protons interact with matter is found to be independent of the material in which the protons stop. Attenuation parameters measured as a part of this dissertation are useful for the shielding design of future proton radiotherapy facilities, provide a benchmark for the current Monte Carlo calculations, and can be used for testing neutron production and transport models. Cost effective shielding can now be implemented without uncertainty and safety concerns resulting from untested calculations. 


\section{Bibliography}

[1] Alsmiller, Jr. R.G., Leimdorfer, M. and Barish, J., "Analytical Representation of Nonelastic Cross Sections and Particle-Emission Spectra from Nucleon-Nucleus Collisions in the Energy Range 25 to $400 \mathrm{MEV}$, ORNL4046, ORNL April 1967.

(2) Alsmiller, Jr. R.G., Barish, J., Boughner, R.T., and Engle, W. W., "Shielding Calculations for a $200 \mathrm{MeV}$ Proton Accelerator", ORNL-4396, Dec. 1968.

(3) Alsmiller, Jr. R.G., Mynatt, F.R., Barish, J., and Engle, W.W. Jr., "Shielding Against Neutrons in the Energy Range 50 to $400 \mathrm{MeV",} \mathrm{ORNL-TM.}$ 2554, March 31, 1969 also Nucl. Instr. Meth. 72 213, 1969.

(4) Alsmiller, Jr. R.G., Mynatt, F.R., Barish, J., and Engle, W.W. Jr., "High Energy ( $<400-\mathrm{MeV})$ Neutron Transport Using the Method of Discrete Ordinates", Nucl. Sci. Eng. 36 251, 1969.

[5] Alsmiller, Jr. R.G., Armstrong, T.W., and Coleman, W.A., "The Absorbed Dose and Dose Equivalent form Neutrons in the Energy Range 60 to 3000 $\mathrm{MeV}$ and Prutons in the Energy Range 400 to $3000 \mathrm{MeV}$ ", ORNL.TM. 2924, Revised July 1970.

[6] Alsmiller, Jr. R.C., and Hermann, O.W., "Calculation of the Neutron Spectra from Proton-Nucleus Nonelastic Collisions in the Energy Range 15 to $18 \mathrm{MeV}$ and Comparison with Experiment", Nucl. Sci. Eng. 40 254, 1970.

[7] Alsmiller, Jr. R.G., and Barish, J., "Shielding Against the Neutrons Produced When $400 \mathrm{MeV}$ Electrons are Incident on a Thick Copper Target", ORNL-TM-4060 also Particle Accelerator's 5, 155, 1973.

[8] Alsmiller, Jr. R.G., Santoro, R.T. and Barish J., "Shielding Calculations for a $200-\mathrm{MeV}$ Proton Accelerators and Comparisons with Experimental Data", ORNL-TM.475/, February 1975, also Accelerators 7, 1, 1975.

[9] Alsmiller, Jr. R.G., Barish, J., Barnes, J.M., and Santoro R.T., "Calculated Neutron Production by 190 to 268 -MeV Protons in a Water-Cooled Tantalunn , Nucl. Sci. Eng. 80 452, 1983. 
[10] Anderson, H.H., and Zeigler, J.F., Hydrogen: Stopping Powers and Ranges in All Elements, (Pergamon Press, Elmsford New York 1977).

[11] Armstrong, T.W., and Barish, J., "Calculation of the Residual- Photon Dose Rate Induced in Iron by 200-MeV Protons", Nucl. Sci. Engr. 38271 1969.

[12] Armstrong, T.W., and Barish, J., "Calculation of the Residual- Photon Dose Rate Due to the Activation of Concrete by Neutrons from a $3-\mathrm{GeV}$ Proton Beam in Iron", Nucl. Sci. Eng. 38 265, 1969.

[13] Aimstrong, T.W., and Barish, J., "Reduction of the Residual Photon Dose Rate Around High-Energy Proton Accelerators", Nucl. Sci. Engr. 40 129, 1970.

[14] Armstrong, T.W., and Bishop, B.L., "Calculation of the Absorbed Dose and Dose Equivalent Induced by Medium-Energy Neutrons and Protons and Comparison with Experiment", Radiation Research 47 581, 1971.

[15] Armstrong, T.W., and Chandler K.C., "The High-Energy Transport Code HETC", Nucl. Sci. Engr. 49 110, 1972.

[16] Armstrong, T.W., Alsmiller, Jr. R.G., Chandler, K.C.., and Bishop, B.L., "Monte Carlo Calculations of High-Energy Nucleon-Meson C'ascades and Comparison with Experiment", ORNL-TM-3667 also Nucl. Sici. Eng. 49 82,1972 .

[17] Armstrong, T.W., and Chandler, K.C., "Calculation of the Absorbed Dose and Dose Equivalent from Protons and Neutrons in the Energy Range forn 3.5 to $1.0 \mathrm{TeV}$ ", ORNL-TM-3758, Oak Ridge National Laboratory, 1972.

[18] Armstrong, T.W., and Colborn, B.L., "A Thick-Target Radiation Transport Computer Code for Low-Mass Heavy Ion Beams", Nucl. Inst. and Meth. 169161.

[19] Armstrong, T.W., Cioth, P., Filges, D., Neef. R.D., and Schaal, H., "Nuclear Data Status and Needs For Spallation Physics Investigations", Nuclear Data for Basic and Applied Science: Volume 2 Young, Brown et al. editors. (Gordon and Breach Science Publishers 1985).

[20] Attix, F.H., Introduction of Radiological Physics and Radiation Dosimetry, (J. Wiley and Sons, New York, 1986).

[21] Awschalom, M., "Radiation Shielding for $250 \mathrm{MeV}$ Protons", Fermilab TM-1354 1985. 
[22] Ban, S., Hirayama, H., and Katoh,K., "Measurement of Secondary Neutron Fluxes Around Beam Stop for $500 \mathrm{MeV}$ Protons", Nucl. Inst. Meth. 184 $409,1981$.

(23] Belogorlov, E.A., Golovachik, V.T., Lebedev, V.N., and Potjomkin, E.L., "Depth Dose and Depth Dose Equivalent Data for Neutrons from Thermal up to Several TeV", Nucl. Instrum. Meth, 199, 563, 1982.

[24] Bertini, H.W., "Low-Energy Intranuclear Cascade Cialculation" Phys. Rev. 131 1801, 1963, with erratum Phys Rev. 138, AB2, 1965, ORNL-TM-1225 1965, ORNL-TM-3844 1966, Trans. Am. Nucl. Soc., Wash., D. C.., Nov. $15-18,1965$, p. 634.

[25] Bertini, H.W., "Monte Carlo Calculations on Intranuclear Cascades", ORNL-33831963.

(26) Bertini, H.W., "Effect of Error on Results of a Low-Energy Intranuclear Cascade Calculation", ORNL-3786, ORNL 1966.

[27] Bertini, H.W., "Results from Low-Energy Intranuclear-Cascade Calculation", Nucl. Phys. 87 138, 1966.

[28] Bertini, H.W., "Secondary Particle Spectra from the Interaction of 30- 340$\mathrm{MeV}$ Protons on Complex. Nuclei: Experimental Data and Comparisons With Theory", Phys. Rev. $1624976,1967$.

(29) Bertini, H.W., "Intranuclear-Cascade Calculation of the Secondary Nucleon Spectra from Nucleon-Nucleus Interactions in the Energy Range 340 to $2900 \mathrm{MeV}$ and Comparisons with Experiment", Phys. Rev. 188 1711, 1969 .

[30] Bertini, H.W., and Guthrie, M.P., "Results for Medium-Energy Intranuclear-Cascade Calculation", Nucl. Phys. A169 670, 1971.

[31] Bertini, H.W., "Comparison of Predictions from Two Intranuclear- Cascade Models with Measured Secondary Proton Spectra at Several Angles from 62- and 92-MeV Protons on Various Elements", ORNL-TM-4638, 1974.

[32] Boag, J.W., In Radiation Dosimetry (Attix, Roesch and Tochilin, eds.), II, 1-72 (Academic Press, New York, 1966).

[33] Braid, T. H., Rapids, R. F., Siemssen, R. H., Tippie, J. W., and O'Brien, K., "Calculation of Shielding for Large Cyclotrons" IEEE Trans. Nucl. Science NS-18 821, 1971. 
[34] Briesmeister, J.F., editor, "MCNP - A General Monte Carlo code for Neutron and Photon Transport", LA-7986-M Revision 2, Los Alamos National Laboratory, 1986.

[35] Broome, T.A., Perry, D.R., and Stapleton, G.B., "Particle Distribution around a Copper Beam Stop for $72-\mathrm{MeV}$ Protons", Health Physics 445 $487,198.3$.

(36) Carter, L.L., "Bulk Shield Design ior Neutron Energies below $50 \mathrm{MeV}$, Nucl. Technol. 3 165, 1983.

[37] Chandler, K.C., and Armstrong, T.W. , "Operating. Instructions for the High-Energy Nucleon-Meson Transport Code HETC", ORNL-4744, ORNL 1972.

[38] Cole, F., Loma Linda University Proton Therapy Facility Operations Man. ual, (Loma I inda Unversity Medical Cienter, Loma Linda, California, Draft, Aug. 1989).

[39] Coleman, W.A., and Armstrong, T.W., "NMTC: A Nucleon-Meson Transport Code", Nucl. Sci. Eng. 43 353, 1971.

[40] Cowling, A.R., and Waker, A.J., "Experimental Studies of a Large-Volume Tissue Equivalent Proportional Counter", in Radiation Protection Dosime. try, Volume 13, No. 1-4 pp. 353-356 1985.

(41) Deluca, Jr., P.M., Torti, R.P., Chenevert, G.M., Detorie, N.A., Tesmer, J.R., and Kelsey, C.A., "Performance of a Gas Target Neutron Source for Radiotherapy", Phys. Med. Bio. 23 876, 1978.

[42] Deluca, Jr. P.M., Schell, M.C., Pearson, D.W., Higgins, P.D. and Attix, F.H., "Performance characteristics of A 150 plastic-equivalent gasses in A150 plastic proportional counters for $14.8-\mathrm{MeV}$ neutrons", Medical Physics 11 4, 449, 1984.

[43] DeLuca, Jr. P.M., Private Communication, April 1990.

[44] Distenfeld, C.H., "Shielding Measurements-200 MeV Linac", BNL-18095, BNL 1973.

(45) Elwyn, A.J., and Cossairt, J.D., "A Study of Neutron Leakage Through An Fe Shield at An Accelerator", Health Physics 516 723, 1986.

(46] Engle, Jr. W.W., "A Users Manual for ANISN, a One Dimensional Discrete Ordinates Transport Code with Anisotropic Scattering", Report Kr-169\%, Computing Technology Center, Union Carbide Corporation, 1967. 
[47] Fano, U., "Note on the Bragg-Gray cavity principle for measuring energy dissipation", Radiat. Res. 1, 237, 1954.

[48] Fasso, A., and Hofert, M., "Distribution of Secondary Particles Around Various Targets Exposed to $50 \mathrm{MeV}$ Protons", Nucl. Instr. Meth. 133 213, 1976.

[49] Freeman, W.S., "Shielding Walls in IB1 for Loma Linda Accelerator", R.P. Note No. 71, FERMILAB Technical Note, May 2, 1988.

[50] Freeman, W.S., "Shielding for the Loma Linda Accelerator in IB1- Experimental Area". R.P. Note No. 74, FERMILAB Technical Note, June 8, 1988.

[51] Fullwood, R.R., Cramer, J.D., Haarmann, R.A., Forrest, R.P., and Schrandt, R. G., "Neutron Production by Medium-Energy Protons on Heavy Metal Targets", Los Alamos Scientific Laboratory, LA-4789 1972.

[52] Guthrie, M.P., "EVAP-4: Another Modification of a Code to Calculate Particle Evaporation from Excited Compound Nuclei", ORNL-TM-3119 1970.

[53] Hagan, W.K., Colborn, B.L., and Armstrong, T.W., "Radiation Shielding Calculations for the Loma Linda Proton Therapy Facility", $S A I C$, 87/107. 1987.

(54) Hagen. W.K., Colborn, B.L., Armstrong, T.W., and Allen, M., "Radiation Shielding Calculations for a $70-250 \mathrm{MeV}$ Proton Therapy Facility", Nucl. Sci. Eng. 98 172, 1988.

[55] Higgins, B., "User's Manual for Microprocessor-Based SWIC Scanner", FNAL-TM-868, 1979.

[56] Hungerford, H.E., Honig, A., Desov, A.E., DuBois, F., and Davis, H.S., "Concretes, Cements, Mortars, and Grouts", In Engineering Compendium on Radiation Shielding, (R.G. Jaeger, E.P Blizard, A.B. Chilton, M. Grotenhuis, A. Honig, Th.A. Jaeger, and H.H. Eisenlohr eds.), II, Section 9.1.12, (Springer-Verlag, New York 1975).

[57] International Atonic Energy Agency, "Radiological Safety Aspects of the Operation of Proton Accelerators", Technical Reports Series No. 983 , (International Atomic Energy Agency, Vienna, 1988).

[58] International Conmission on Radiological Protection, "Statement from the 1985 Paris Meeting of the International Commission on Radiological Protection", ICRP Publication No. 45, (Perniagon Press, 1985). 
[59| International Commission on Radiological Protection, "Data for Use in Protection Against External Radiation", ICRP Publication No. 51, (Permagon Press, 1987).

[60] International Commission on Radiation Units and Measurements, "Radiation Protection Instrumentation and Its Application", ICRU Report 20 1971.

(61) International Commission on Radiation Units and Measurements, "Neutron Dosimetry for Biology and Medicine", ICRU Report 261977.

(62) International Commission on Radiation Units and Measurements, "Average Energy Required to Produce and Ion Pair", ICRU Report 311979.

[63] International Commission on Radiation Units and Measurements, "Microdosimetry", ICRU Report 361983.

[64] Internetional Commission on Radiation Units and Measurements, "The Quality Factor in Radiation Protection", IC'RU Report. 101986.

(65) Irving, D.C., Freestone, Jr. R.M., and Kam, F.B.K., "O5R, A General Purpose Monte-Carlo Neutron Transport Code", ORNL-36.9 1965.

[66] Irving, D.C., Alsmiller, Jr. R.G., and Moran, H.S., "Tissue-to-dose Conversion Factors for Neutrons with Energies from 0.5 to $61 \mathrm{MeV}$ ", Nucl. Instrum. Meth. 51, 129, 1967.

[67] Janni, J.F., "Proton Range Energy Tables", Alomic Data and Nuclear Data Tables 27, Numbers 4/5, (1982).

[68] Kinney, W.E., "The Nucleon Transport Code, NTC", ORNL-36101964.

[69] Lederer, C.M., and Shirley, V.S., Table of Isotopes-Seventh Edition, (Wiley Press, New York, 1978).

[70] Lone, M.A., Jones, R.R., and Okazaki, A., et al. "Total Neutron Yield from 100-MeV Protons on Pb and "Li", Nucl. Instr. Meth. 214 333.3, 1983.

[71.] Maerker, R.E., and Muckenthaler, F.J., "Calculation and Measurement of" the Fast Neutron Differential Dose Albedo for Concrete", Nucl. Sci. Eng. $22455,1965$.

[72] Marshall, I.R., and Williamson, F.S., "Microdosimetric Spectra Measurements of Janus Neutrons", in Radiation Protection Dosimetry, Volume 1.3, No. 1-4 pp. 111-115 1985. 
[73] Meier, M.M., Goulding, C.A., Morgan, G.L., and Ullmann J.L., "Neutron Yields from Stopping-Length Targets and Near Stopping-Length Targets for $256 \mathrm{MeV}$ Protons", Los Alamos Report LA-11286-MS 1988 and submitted to Nucl. Sci. Eng.

[74] Meier, M.M., Amian, W.B., Clark, D.A., Goulding, C.A., McClelland, J.B., Morgan, G.L., and Moss,C.E., "Differential Neutron Production Cross Sections and Neutron Yields from Stopping-Length Targets for 113MeV Protons", Los Alamos Report LA-11518-MS 1989.

(75] Meier, M.M., Amian, W.B., Goulding, C.A., Morgan, G.L., and Moss,C.E., "Differential Neutron Production Clross Sections and Neutron Yields from Stopping-Length Targets for 256-MeV Protons", Los Alamos Report LA. 11656-MS 1989.

(76) Menzel, H.G., Buhler, G., and Schuhmacher, H., "Investigation of Basic Uncertianties in the Experimantal Determination of Microdosimetric Data", in Proc. 8th Symp. on Microdosimetry, eds. J. Booz and H.G. Ebert, EUR 8395 (Commission of the European Communities, Brussels) pp. 1061-1072 (1983).

[77] Menzel, H.G., Pihet, P., Folkerts, K.H., Dahman, P., and Grillmair, R.E., "Dosimetry Research Using Low Pressure Proportional Counters for Neutrons with Energies Up to $60 \mathrm{MeV}$ ", in Radiation Protection Dosimetry, Volume 23, No. 1/4 pp. 389-392 1988.

[78] Nakamura, T., Yoshida, M., and Shin, K., "Spectral Measurements of Ne11trons and Photons from Thick Targets of $\mathrm{C}, \mathrm{Fe}, \mathrm{Cu}$, and $\mathrm{Pb}$ by $52 \mathrm{MeV}$ Protons", Nucl. Inst. Meth. $151493,1978$.

[79] Nakamura, T., Fujii, M., and Shin, K., "Neutron Production from Thick Targets of Carbon, Iron, Copper and Lead by Protons 30 to $50 \mathrm{MeV}$ Protons", Nucl. Sci. Eng. 83 444, 1983.

[80] National Council on Radiation Protection and Measurements, "Basic Radiation Protection Criteria", NCRP 39, Jan. 1971.

[81] Neufeld, J., Snyder, W.S., Turner, J.E., Wright, H., Wheatley, B.M., and Wyckoff, H.O., "Radiation dose from Neutrons and Protons in the Energy Rannge from $400 \mathrm{MeV}$ to $2 \mathrm{GeV",} \mathrm{Health} \mathrm{Physics,} \mathrm{17,} \mathrm{449,} 1969$.

182] O'Brien, K., "Tables for the Determination of the Lateral Shielding Requirements of High Energy Electron and Proton Accelerators", IEEE Trans. Nucl. Sci. NS-16 568, 1969. 
[83] Patterson, H.W., and Thomas, R.H., Accelerator Health Physics (Academic Press, NY 1973).

[84] Pearlstein, S., "Systematics of Neutron Emission Spectra from HighEnergy Proton Bombardment", Nuclear Science and Engineering 95 116$127,1987$.

[85] Pihet, P., Gueulette, J., Menzel, H.G., Grillmair, R.E., and Wambersie, A., "Use of Microdosimetric Data of clinical Relevance in Neutron Therapy Planning", in Radiation Protection Dosimetry, Volume 23, No. 1/4 pp. 471-474 1988 .

[86] Prael, R.E., and Bozoian, M., "Adaptation of the Multistage Preequilibrium Model for the Monte Carlo Method (I)", LA-UR-88-9298, Los Alamos National Laboratory, 1988. Prael, R.E., and Bozoian, M., "Adaptation of the Multistage Preequilibrium Model for the Monte Carlo Method (II)", Los Alamos National Laboratory, 'to be published).

[87] Prael, R.E., and Lichtenstein, H., "Users Guide to LCS: The LAHET Code System", LA.UR.89.3014, Los Alamos National Laboratory, 1989.

[88] Prael, R.E., "LAHET Benchmark Calculation of Neutron Yield from Stopping-Length Targets for $113 \mathrm{MeV}$ and $256 \mathrm{MeV}$ Protons", LA.UR90-1620, Los Alamos National Laborztory, 1990.

[89] Raju, M.R., Lyman, J.T., Brustad, 'T., and Tobias, C.A., "Heavy Charged Particle Beams", in Radiation Dosimetry (Attic, Roesch and Tochlin, eds.), III, 151 (Academic Press, New York, 1969).

[90] Rossi, H.H., and Rosenzweig, W., "A Device for the Measurement of Dose as a function of Specific Ionization", Radiology 64 pp.404-411, 1955.

[91] Roussin, R.W., Alsmiller, Jr. R.G., and Barish, J., "Calculations of the Transport of Neutrons and Secondary Gamma Rays Through Concrete for Jucident Neutrons in the Energy Range 15 to $75 \mathrm{MeV}$ ", Nucl. Eng. Design $24250,1973$.

[92] Ryabov, Y.V., Matushko, G.K., and Slastnikov, V.N., "Neutron Yield form 250-MeV Protons on Lead", Z. Phys. A 311 363, 1983.

[93] Schrewe, U.J., Brede, H.J., Pihet, P., and Menzel, H.C.., "On The Calibration of Tissue-Equivalent Proportional Connters with Built-In Alpha Particle Sources", in Radiation Protection Dosimetry, Volume 2?, No. 1/4 pp. 249-252 1988. 
(94) Schuhmacher, H., Menzel, H.G., Buhler, G., and Alberts, W.G., "Exper" imental Basis for Optimisation of the Wall Thickness of Microdosimetric Counters in Radiation Protection", in Radiation Protection Dosimetry, Volume 13, No. 1-4 pp. 341-3541985.

(95) Shaw, K.B., and Stevenson, G.R., "Radiation Studies around Extracted Proton Beams at NIMROD", IEEE. Trans. Nucl. Sci. NS-16 570, 1969.

[96] Schimmerling, W., and Awschalom, M., "Neutron Flux in a Labyrinth Due to $3 \mathrm{GeV}$ Proton Beam Incident on Lead", Proc. 1969 Particle Accel Conf., Wash. 1969, Vol. I, p. 604.

[97] Smathers, J.B., Otte, V.A., Smith, A.R., Almond, P.R., Attix, F.H., Spokas, J.J.", Quam, W.M., and Goodmann, L.J., "Composition of A-150 tissue-equivalent plastic", Medical Physics, 4, 74, 1977.

(98] Smith, H.A., "Shielding Calculations and Beam Dump Shielding Design Considerations for the Indiana University Cyclotron Facility", Indiana University University Cyclotron Facility Internal Report no. 74 6, May 1974.

199] Srdoc, D., "Experimental Technique of Measurement of Microscopic Energy Distribution in Irradiated Matter Uing Rossi Counter", Radiation Research 43, 302, 1970.

[100] Stevenson, G.R., Kuei-Lin, L., and Thomas, R.H., "Determination of the Transverse Shielding for Proton Accelerators Using The Moyer Model", Health Physics 431 13-29, 1982.

(101) Stevenson, G.R., "Neutron Attenuation in Labyrinths, Ducts and Penetrations at High-Energy Proton Accelerators", Theory and Practice in Ra-

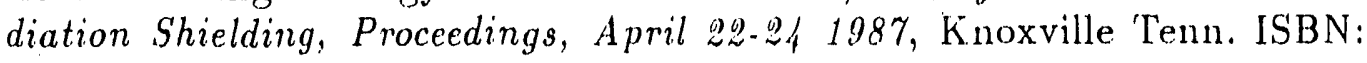
0-89448-132-?

[102] Stevenson, G.R., CERN Report TIS-RP/173, 1986.

[103| Taylor, J.R., An Introduction to Error Analysis, (University Science Books, California), 1982.

(104] Tesch, K., "Comments on the Transverse Shielding of Proton Accelerators", Health Physics 44791983.

[105] Tesch, K., "A Simple Estimation of the Lateral Shielding For Proton Accelerators in the Energy Range 50 to $1000 \mathrm{MeV}$ ", in Radiation Protection Dosimetry, Volume II, No. 3 pp. 162-167 1985. 
[1.06) Varma, M.N., "Calibration of Proportional Counter in Microdosimetry", in Proc. 8th Symp; on Microdosimetry, eds. J. Booz and H.G. Ebert, EUR 8395 (Commission of the European Communities, Brussels) pp. 1051-1059 (1983).

[107] Wacinter, J.W., Burrus, W.R., Gibson, W.A., "Neutron and Proton Spectra from Targets Bombarded by $160 \mathrm{MeV}$ Protons", Phys. Rev. 1614971 , 1967 .

[108] West, D., and Wood, E., "Neutron Yield from Protons and Deuterons of Energies around $1 \mathrm{GeV}$ Totally Absorbed in Lead", Can. J. Phys. 49 2061, 1971.

[109] Yariv, Y., and Fraenkel, Z., "Intranuclear Cascade Calculation of Heavy Ion Interactions" Phys. Rev. C, 20, 2227, 1979.

[110] Yariv, Y., and Fraenkel, Z., "Intranuclear Cascade Calculation of Heavy Ion Collisions: Effect of Interaction Between C'ascade Particles" Phys. Rev. C, 24, 488, 1981.

111) Zazula, J.M., and Tesch, K., "Analysis of the Transverse Shielding Problem at Proton Accelerators using a Hadronic Cascade Code with Low Energy Particle Modules", Nucl. Instrum. Meth., A286, 279, 1990.

[112] Zerby, C.D., and Kinney, W.E., "Calculated Tissue-to-dose Conversion Factors for Nucleons Below $400 \mathrm{MeV}$ ", Nucl. Instrum. Meth. 36, 125, 1965. 

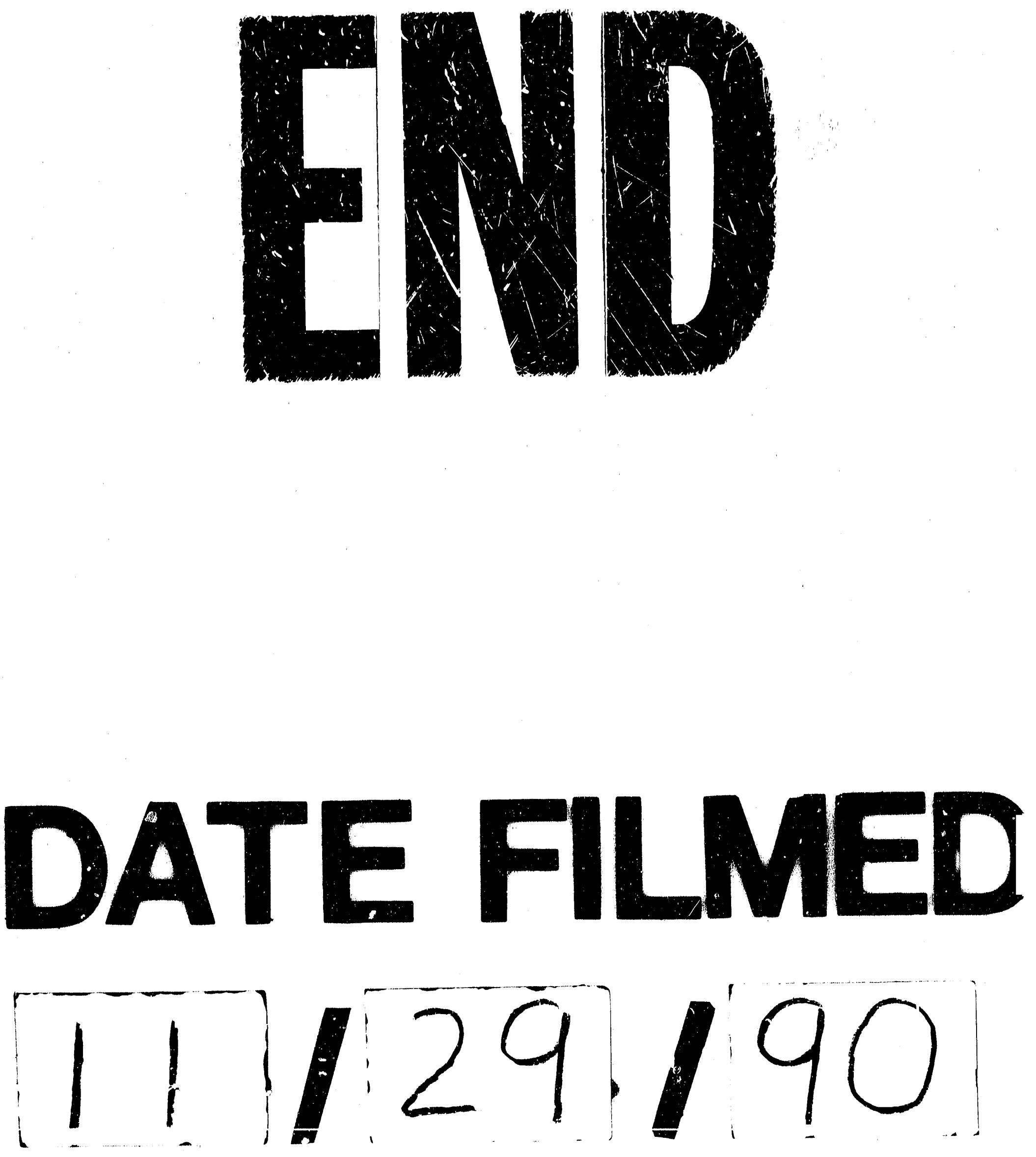
